\title{
Galois actions on homotopy groups of algebraic varieties
}

\author{
JONATHAN P PRIDHAM
}

\begin{abstract}
We study the Galois actions on the $\ell$-adic schematic and Artin-Mazur homotopy groups of algebraic varieties. For proper varieties of good reduction over a local field $K$, we show that the $\ell$-adic schematic homotopy groups are mixed representations explicitly determined by the Galois action on cohomology of Weil sheaves, whenever $\ell$ is not equal to the residue characteristic $p$ of $K$. For quasiprojective varieties of good reduction, there is a similar characterisation involving the Gysin spectral sequence. When $\ell=p$, a slightly weaker result is proved by comparing the crystalline and $p$-adic schematic homotopy types. Under favourable conditions, a comparison theorem transfers all these descriptions to the Artin-Mazur homotopy groups $\pi_{n}^{\text {ét }}\left(X_{\bar{K}}\right) \otimes_{\widehat{\mathbb{Z}}} \mathbb{Q}_{\ell}$.
\end{abstract}

\section{Introduction}

In [2], Artin and Mazur introduced the étale homotopy type of an algebraic variety. This gives rise to étale homotopy groups $\pi_{n}^{\text {ét }}(X, \bar{x})$; these are pro-finite groups, abelian for $n \geq 2$, and $\pi_{1}^{\text {ét }}(X, \bar{x})$ is the usual étale fundamental group. In [49, Section 3.5.3], Toën discussed an approach for defining $\ell$-adic schematic homotopy types, giving $\ell$-adic schematic homotopy groups $\varpi_{n}(X, \bar{x})$; these are (pro-finite-dimensional) $\mathbb{Q}_{\ell}$-vector spaces when $n \geq 2$. In [33], Olsson introduced a crystalline schematic homotopy type, and established a comparison theorem with the $p$-adic schematic homotopy type.

Thus, given a variety $X$ defined over a number field $K$, there are many notions of homotopy group:

- for each embedding $K \hookrightarrow \mathbb{C}$, both classical and schematic homotopy groups of the topological space $X_{\mathbb{C}}$;

- the étale homotopy groups of $X_{\bar{K}}$;

- the $\ell$-adic schematic homotopy groups of $X_{\bar{K}}$;

- over localisations $K_{\mathfrak{p}}$ of $K$, the crystalline schematic homotopy groups of $X_{K_{\mathfrak{p}}}$. 
However, despite their long heritage, very little was known even about the relation between étale and classical homotopy groups, unless the variety is simply connected.

The étale and $\ell$-adic homotopy types carry natural Galois actions, and the main aim of this paper is to study their structure. In many respects, the analogous question for $X_{\mathbb{C}}$ has already been addressed, with Katzarkov, Pantev and Toën [22] and the author [34] describing mixed Hodge structures on the classical and real schematic homotopy types. In [37], the author introduced a new approach to studying nonabelian cohomology and schematic homotopy types of topological spaces. Its primary application was to transfer cohomological data (in particular mixed Hodge structures) to give information about homotopy groups. The bulk of this paper is concerned with adapting those techniques to pro-simplicial sets. This allows us to study Artin-Mazur homotopy types of algebraic varieties, and to translate Lafforgue's Theorem and Deligne's Weil II theorems into statements about homotopy types. We thus establish arithmetic analogues of the results of [34], with Galois actions replacing mixed Hodge structures.

The main comparison results are Proposition 1.39 (showing when étale homotopy groups are pro-finite completions of classical homotopy groups), Theorem 3.40 (describing $\ell$-adic schematic homotopy groups in terms of étale homotopy groups), and Proposition 7.26 (comparing $p$-adic and crystalline homotopy groups).

If $X$ is smooth or proper and normal, then Corollary 6.7 shows that the Galois actions on the $\ell$-adic schematic homotopy groups are mixed, with Remark 6.9 indicating when the same is true for étale homotopy groups. Corollaries 6.11 and 6.16 then show how to determine $\ell$-adic schematic homotopy groups of smooth varieties over finite fields as Galois representations, by recovering them from cohomology groups of smooth Weil sheaves, thereby extending the author's paper [38] from fundamental groups to higher homotopy groups, and indeed to the whole homotopy type. Corollaries 7.4 and 7.36 give similar results for $\ell$-adic and $p$-adic homotopy groups of varieties over local fields.

The structure of the paper is as follows.

In Section 1, we recall standard definitions of pro-finite homotopy types and homotopy groups, and then establish some fundamental results. Proposition 1.29 shows how Kan's loop group can be used to construct the pro-finite completion $\hat{X}$ of a space $X$, and Proposition 1.39 describes homotopy groups of $\hat{X}$.

Section 2 reviews the pro-algebraic homotopy types of [37], with the formulation of multipointed pro-algebraic homotopy types from [34], together with some new material on hypercohomology.

We adapt these results in Section 3 to define nonabelian cohomology of a variety with coefficients in a simplicial algebraic group over $\mathbb{Q}_{\ell}$. The machinery developed 
in [37] applies to give a pro- $\mathbb{Q}_{\ell}$-algebraic homotopy type, which is a nonnilpotent generalisation of the $\mathbb{Q}_{\ell}$-homotopy type of Weil II (see Deligne [5]). Its homotopy groups are $\ell$-adic schematic homotopy groups, and Theorem 3.40 gives conditions for relating these to étale homotopy groups. Explicitly, if $\pi_{1} X$ is algebraically good (see Definition 3.35), and the higher homotopy groups have finite rank, then the higher homotopy groups of the pro- $\mathbb{Q}_{\ell}$-algebraic homotopy type are just $\pi_{n}^{\text {ét }} X \otimes_{\widehat{\mathbb{Z}}} \mathbb{Q}_{\ell}$. For complex varieties, we also compare the pro-algebraic homotopy types associated to the étale and analytic topologies.

Section 4 contains technical results showing how to extend the machinery of Section 3 to relative and filtered homotopy types. The former facilitate $p$-adic Hodge theory, while the latter are developed in order to study quasiprojective varieties. We also explore what it means for a pro-discrete group to act algebraically on a homotopy type. In Section 5, we investigate properties of homotopy types endowed with algebraic Galois actions.

In Section 6, the techniques of [38] for studying Galois actions on algebraic groups then extend the finite characteristic results of the author in [35] to nonnilpotent and higher pro$\mathbb{Q}_{\ell}$-algebraic homotopy groups. The results are similar to [34], substituting Frobenius actions for Hodge structures. Over finite fields, Theorem 6.10 uses Lafforgue's Theorem and Deligne's Weil II theorems to show that the pro- $\mathbb{Q}_{\ell}$-algebraic homotopy type of a smooth projective variety is formal - this means that it can be recovered from cup products on cohomology of local systems. For quasiprojective varieties, Corollary 6.15 establishes a related property we call quasiformality, which is analogous to Morgan's description of the rational homotopy type [31] in terms of the Leray spectral sequence.

Section 7 then addresses the same question, but over local fields. In unequal characteristic, smooth specialisation suffices to adapt results from finite characteristic for varieties with good reduction. In equal characteristic, we show how pro- $\mathbb{Q}_{p}$-algebraic homotopy types relate to the framework of $p$-adic Hodge theory. Proposition 7.26 is a reworking of Olsson's nonabelian $p$-adic Hodge theory, and this has various consequences for Galois actions on Artin-Mazur homotopy types (Theorems 7.28-7.35). Explicitly, the homotopy type becomes formal as a Galois representation only after tensoring with the ring $B_{\text {cris }}^{\sigma}$ of Frobenius-invariant periods, which means that the Hodge filtration is the only really new structure on the relative Malcev homotopy type (Remarks 7.37(2)).

Acknowledgements I would like to record my sincere thanks to the team of referees for patiently reading through the manuscript. As well as identifying numerous errors, their suggestions have greatly improved the exposition.

This work was supported by Trinity College, Cambridge; and by the Engineering and Physical Sciences Research Council grant number EP/F043570/1. 


\section{Pro-finite homotopy types}

Definition 1.1 Let $\mathbb{S}$ be the category of simplicial sets, and take $s$ Gpd to consist of those simplicial objects in the category of groupoids whose spaces of objects are discrete (ie sets, rather than simplicial sets).

Let Top denote the category of compactly generated Hausdorff topological spaces.

Definition 1.2 Given $G \in s \mathrm{Gpd}$, we define $\pi_{0} G$ to be the groupoid with objects Ob $G$, and morphisms $\left(\pi_{0} G\right)(x, y)=\pi_{0} G(x, y)$.

Definition 1.3 A map $f: X \rightarrow Y$ in Top is said to be a weak equivalence if it gives an isomorphism $\pi_{0} X \rightarrow \pi_{0} Y$ on path components, and for all $x \in X$, the maps $\pi_{n}(f): \pi_{n}(X, x) \rightarrow \pi_{n}(Y, f x)$ are all isomorphisms.

We give $\mathbb{S}$ the model structure of Goerss and Jardine [11, Theorem V.7.6]; in particular, a map $f: X \rightarrow Y$ in $\mathbb{S}$ is said to be a weak equivalence if the map $|f|:|X| \rightarrow|Y|$ of topological spaces is so, where $|\cdot|$ is the realisation functor of [11, Section I.2]. Likewise, for $x \in X_{0}$ we write $\pi_{n}(X, x):=\pi_{n}(|X|, x)$.

A map $f: G \rightarrow H$ in $s \mathrm{Gpd}$ is a weak equivalence if the map $\pi_{0} G \rightarrow \pi_{0} H$ is an equivalence, and for all objects $x \in \mathrm{Ob} G$, the maps $\pi_{n}(G(x, x)) \rightarrow \pi_{n}(H(f x, f x))$ are all isomorphisms.

For each of these categories, we define the corresponding homotopy categories $\operatorname{Ho}(\mathbb{S})$, $\mathrm{Ho}(\mathrm{Top}), \mathrm{Ho}(s \mathrm{Gpd})$ by localising at weak equivalences.

Note that there is a functor from Top to $\mathbb{S}$ which sends $X$ to the simplicial set

$$
\operatorname{Sing}(X)_{n}=\operatorname{Hom}_{\mathrm{Top}}\left(\left|\Delta^{n}\right|, X\right) .
$$

This is right adjoint to realisation, and these functors are a pair of Quillen equivalences, so become quasi-inverse on the corresponding homotopy categories. From now on, we will thus restrict our attention to simplicial sets.

Definition 1.4 Given $G \in s \mathrm{Gpd}$, define the category $\mathbb{S}_{G}$ of $G$-spaces to consist of simplicial representations of $G$. Explicitly, $X \in \mathbb{S}_{G}$ consists of $X(a) \in \mathbb{S}$ for each $a \in \mathrm{Ob} G$, together with maps $G(a, b) \times X(b) \rightarrow X(a)$, satisfying the obvious associativity and unit axioms. 
Definition 1.5 Recall from [11, Section V.4] that for $G \in s G$ pd, the $G$-space $W G$ is defined by

$$
(W G)_{n}(x)=\coprod_{y_{n}, \ldots, y_{0} \in \mathrm{Ob} G} G_{n}\left(x, y_{n}\right) \times G_{n-1}\left(y_{n}, y_{n-1}\right) \times \ldots \times G\left(y_{1}, y_{0}\right)
$$

with operations

$$
\begin{gathered}
\partial_{i}\left(g_{n}, g_{n-1}, \ldots, g_{0}\right)=\left\{\begin{array}{cc}
\left(\partial_{i} g_{n}, \partial_{i-1} g_{n-1}, \ldots,\right. & i<n, \\
\left.\left(\partial_{0} g_{n-i}\right) g_{n-i-1}, g_{n-i-2}, \ldots, g_{0}\right) & \\
\left(\partial_{n} g_{n}, \partial_{n-1} g_{n-1}, \ldots, \partial_{1} g_{1}\right) & i=n,
\end{array}\right. \\
\sigma_{i}\left(g_{n}, g_{n-1}, \ldots, g_{0}\right)=\left(\sigma_{i} g_{n}, \sigma_{i-1} g_{n-1}, \ldots, \sigma_{0} g_{n-i}, \text { id }, g_{n-i-1}, \ldots, g_{0}\right),
\end{gathered}
$$

and for $h \in G_{n}(z, x)$ and $\left(g_{n}, g_{n-1}, \ldots, g_{0}\right) \in(W G)(x)$,

$$
h\left(g_{n}, g_{n-1}, \ldots, g_{0}\right)=\left(h g_{n}, g_{n-1}, \ldots, g_{0}\right) .
$$

Note that $W G(x)$ is contractible for each $x \in \mathrm{Ob} G$.

Definition 1.6 As in Goerss and Jardine [11, Chapter V.7], there is a classifying space functor $\bar{W}: s \mathrm{Gpd} \rightarrow \mathbb{S}$, given by $\bar{W} G=G \backslash W G$, the coinvariants of the $G$-action. This has a left adjoint $G: \mathbb{S} \rightarrow s \mathrm{Gpd}$, Dwyer and Kan's loop groupoid functor [7], and these form a pair of Quillen equivalences, so give equivalences $\operatorname{Ho}(\mathbb{S}) \sim \operatorname{Ho}(s \mathrm{Gpd})$. The objects of $G(X)$ are $X_{0}$, and for any $x, y \in X_{0}$, the geometric realisation $|G(X)(x, y)|$ is weakly equivalent to the space of paths from $x$ to $y$ in $|X|$. These functors have the additional properties that $\pi_{0} G(X) \cong \pi_{f}|X|$ (the fundamental groupoid), $\pi_{f}(|\bar{W} G|) \cong$ $\pi_{0} G, \pi_{n}(G(X)(x, x)) \cong \pi_{n+1}(|X|, x)$ and $\pi_{n+1}(|\bar{W} G|, x) \cong \pi_{n}(G(x, x))$. This allows us to study simplicial groupoids instead of topological spaces.

Definition 1.7 If $X \in \mathbb{S}$, then a local system is just a representation of the groupoid $\pi_{f} X$, ie a functor $\pi_{f} X \rightarrow$ Gp from the fundamental groupoid to the category of groups. As in [11, Section VI.5], homotopy groups form a local system $\pi_{n} X$, whose stalk at $x$ is $\pi_{n}(X, x)$.

\subsection{Pro-simplicial $L$-groupoids}

Definition 1.8 Given a set $L$ of primes, we say that an $L$-group is a finite group $G$ for which only primes in $L$ divide its order. We define an $L$-groupoid to be a groupoid $H$ for which $H(x, x)$ is an $L$-group for all $x \in \mathrm{Ob} H$. 
Definition 1.9 Given a category $\mathcal{C}$, recall that the category $\operatorname{pro}(\mathcal{C})$ of pro-objects in $\mathcal{C}$ has objects consisting of filtered inverse systems $\left\{A_{\alpha}\right\}$ in $\mathcal{C}$, with

$$
\operatorname{Hom}_{\operatorname{pro}(\mathcal{C})}\left(\left\{A_{\alpha}\right\},\left\{B_{\beta}\right\}\right)=\underset{\beta}{\lim } \underset{\alpha}{\lim } \operatorname{Hom}_{\mathcal{C}}\left(A_{\alpha}, B_{\beta}\right)
$$

Remark 1.10 A discrete topological space is just a set. Given a pro-set $\left\{X_{\alpha}\right\}$, we can thus take the limit $\lim _{\alpha} X_{\alpha}$ in the category of topological spaces. This functor gives a faithful embedding of pro(Set) into topological spaces, so ${\underset{\lim }{\alpha}}_{\alpha} X_{\alpha}$ is discrete if and only if $\left\{X_{\alpha}\right\}$ lies in the essential image of Set $\rightarrow \operatorname{pro}(\mathrm{Set})$. We will thus refer to the essential image of Set $\rightarrow$ pro(Set) as the discrete objects.

In fact, pro-sets endow a topological structure which cannot be detected by weak equivalences, which is why shape theory is modelled using the category $\operatorname{pro}(\mathbb{S})$, as in Isaksen [19].

Definition 1.11 Given a groupoid $G$ and a set $L$ of primes, define $G^{\wedge} L \in \operatorname{pro}(\mathrm{Gpd})$ by requiring that $G^{\wedge} L$ be the completion of $G$ with respect to all $L$-groupoids $H$. In other words, $G^{\wedge} L$ is an inverse system of $L$-groupoids, with a canonical map $G \rightarrow G^{\wedge} L$ inducing isomorphisms

$$
\operatorname{Hom}\left(G^{\wedge}, H\right) \rightarrow \operatorname{Hom}(G, H)
$$

for all $L$-groupoids $H$.

In particular, $\mathrm{Ob} G^{\wedge} L=\mathrm{Ob} G$ and $G^{\wedge} L(x, x)$ is the pro- $L$ completion of the group $G(x, x)$ (in the sense of Friedlander [10, Section 6]). If $L$ is the set of all primes, we write $\widehat{G}:=G^{\wedge} L$, so $\widehat{G}(x, x)$ is the pro-finite completion of $G(x, x)$ (in the sense of Serre [47, Section 1]).

Note that $G^{\wedge} L$ is a pro- $L$-groupoid in the sense of Definition 1.9. However, beware that a pro-groupoid can be isomorphic to a pro- $L$-groupoid without actually being an inverse system of $L$-groupoids, since $\left\{\Gamma_{\alpha}\right\}_{\alpha \in I} \cong\left\{\Gamma_{\alpha}\right\}_{\alpha \geq \alpha_{0}}$ for any $\alpha_{0} \in I$.

Definition 1.12 Say that a simplicial groupoid $\Gamma$ is a simplicial $L$-groupoid if $\Gamma_{i}$ is an $L$-groupoid for all $i$. Denote the category of such groupoids by $s \mathrm{Gpd}^{L}$.

Definition 1.13 Given a groupoid $\Gamma$, define a disconnected normal subgroupoid $K \triangleleft \Gamma$ to consist of subgroups $K(x) \leq \Gamma(x, x)$ for all $x \in \mathrm{Ob} \Gamma$, with $a K(x) a^{-1} \in K(y)$ for all $a \in \Gamma(y, x)$. 
Note that disconnected normal subgroupoids $K \triangleleft \Gamma$ are in one-to-one correspondence with isomorphism classes of surjections $f: \Gamma \rightarrow H$ for which $\mathrm{Ob} f: \mathrm{Ob} \Gamma \rightarrow \mathrm{Ob} H$ is an isomorphism. The equivalence is given by setting $H(x, y)=\Gamma(x, y) / K(y)=$ $K(x) \backslash \Gamma(x, y)$, and conversely by setting $K(x):=\operatorname{ker}(f: \Gamma(x, x) \rightarrow H(f x, f x))$.

Definition 1.14 Given $\Gamma \in s \mathrm{Gpd}$, define a simplicial disconnected normal subgroupoid $K \triangleleft \Gamma$ to consist of disconnected normal subgroupoids $K_{n} \triangleleft \Gamma_{n}$, closed under the operations $\partial_{i}, \sigma_{j}$.

Definition 1.15 Given $\Gamma \in s \mathrm{Gpd}$, define $\Gamma^{\wedge} L \in \operatorname{pro}\left(s \mathrm{Gpd}^{L}\right)$ to be the inverse system $\{\Gamma / K\}_{K}$, where $K$ ranges over the poset of all simplicial disconnected normal subgroupoids $K \triangleleft \Gamma$ for which $\Gamma / K$ is a simplicial $L$-groupoid.

Given $\Gamma=\left\{\Gamma_{\alpha}\right\}_{\alpha} \in \operatorname{pro}(s \mathrm{Gpd})$, define $\Gamma^{\wedge} L \in \operatorname{pro}\left(s \mathrm{Gpd}^{L}\right)$ by

$$
\Gamma^{\wedge} L=\overleftarrow{\alpha}_{\overleftarrow{\alpha}}^{\lim } \Gamma_{\alpha}^{\wedge^{L}}
$$

where the limit is taken in $\operatorname{pro}\left(s \mathrm{Gpd}^{L}\right)$. This corresponds to saying that $\Gamma^{\wedge_{L}}$ is the pro-object $\left\{\Gamma_{\alpha} / K_{\alpha}\right\}_{\left(\alpha, K_{\alpha}\right)}$ indexed by pairs $\left(\alpha, K_{\alpha}\right)$, for $K_{\alpha} \triangleleft \Gamma_{\alpha}$.

Lemma 1.16 For $\Gamma \in \operatorname{pro}(s \mathrm{Gpd})$ and $A \in \operatorname{pro}\left(s \mathrm{Gpd}^{L}\right)$, the canonical map

$$
\operatorname{Hom}_{\operatorname{pro}\left(s \mathrm{Gpd}^{L}\right)}\left(\Gamma^{\wedge L}, A\right) \rightarrow \operatorname{Hom}_{\operatorname{pro}(s \mathrm{Gpd})}(\Gamma, A)
$$

is an isomorphism.

Proof By the definition of morphisms in pro-categories, it suffices to prove this when $A \in s \mathrm{Gpd}^{L}$. Then $A$ is cofinite in both $\operatorname{pro}\left(s \mathrm{Gpd}^{L}\right)$ and $\operatorname{pro}(s \mathrm{Gpd})$ (ie $\operatorname{Hom}\left(\lim _{\alpha} \Gamma_{\alpha}, A\right) \cong \lim _{\longrightarrow} \operatorname{Hom}\left(\Gamma_{\alpha}, A\right)$ for filtered inverse systems $\left.\left\{\Gamma_{\alpha}\right\}_{\alpha}\right)$, so we may also assume that $\underset{\Gamma}{\leftarrow} \in s \mathrm{Gpd}$.

Now, for any morphism $f: \Gamma \rightarrow A$, the image $H$ is a simplicial $L$-groupoid of the form $H=\Gamma / K$, for $K \triangleleft \Gamma$ a disconnected normal subgroupoid. Therefore

$$
\operatorname{Hom}_{s \mathrm{Gpd}}(\Gamma, A)=\underset{K}{\underset{\mathrm{lim}}{\longrightarrow}} \operatorname{Hom}_{s \mathrm{Gpd}}(\Gamma / K, A)=\operatorname{Hom}_{\mathrm{pro}(s \mathrm{Gpd})}\left(\Gamma^{\wedge} L, A\right),
$$

as required.

Lemma 1.17 For $\Gamma \in \operatorname{pro}(s \mathrm{Gpd})$, the pro-L-groupoid $\left(\Gamma^{\wedge_{L}}\right)_{n}$ is just the pro- $L$ completion of $\Gamma_{n}$. 
Proof Given $A \in \mathrm{Gpd}^{L}$, define $A^{\Delta_{n}}$ (not to be confused with $A^{\Delta^{n}}$ ) to be the simplicial groupoid on objects $\mathrm{Ob} A$ with

$$
A^{\Delta_{n}}(x, y)_{i}:=A(x, y)^{\Delta_{n}^{i}},
$$

with $\partial_{j}:\left(A^{\Delta_{n}}\right)_{i} \rightarrow\left(A^{\Delta_{n}}\right)_{i-1}$ coming from $\partial^{j}: \Delta^{i-1} \rightarrow \Delta^{i}$, and $\sigma_{j}$ coming from $\sigma^{j}: \Delta^{i+1} \rightarrow \Delta^{i}$. Then $A^{\Delta_{n}}$ is clearly an $L$-groupoid, and has the key property that

$$
\operatorname{Hom}_{s \mathrm{Gpd}}\left(\Gamma, A^{\Delta_{n}}\right) \cong \operatorname{Hom}_{\mathrm{Gpd}}\left(\Gamma_{n}, A\right)
$$

for all $\Gamma$.

Taking colimits extends this to all $\Gamma \in \operatorname{pro}(s \mathrm{Gpd})$, and then

$$
\operatorname{Hom}_{\operatorname{pro}\left(s \mathrm{Gpd}^{L}\right)}\left(\Gamma^{\wedge L}, A^{\Delta_{n}}\right) \cong \operatorname{Hom}_{\operatorname{pro}\left(\mathrm{Gpd}^{L}\right)}\left(\left(\Gamma^{\wedge L}\right)_{n}, A\right),
$$

but the left-hand side is just

$$
\operatorname{Hom}_{\operatorname{pro}(s \mathrm{Gpd})}\left(\Gamma, A^{\Delta_{n}}\right) \cong \operatorname{Hom}_{\operatorname{pro}(\mathrm{Gpd})}\left(\Gamma_{n}, A\right),
$$

so $\left(\Gamma^{\wedge} L\right)_{n}$ is the pro- $L$ completion of $\Gamma_{n}$.

Definition 1.18 Given $X=\left\{X_{\alpha}\right\} \in \operatorname{pro}(\mathbb{S})$, define the category of local systems on $X$ to be the direct limit (over $\alpha$ ) of the categories of local systems on $X_{\alpha}$ (in the sense of Definition 1.7).

Remark 1.19 Our motivation for working with pro(S) comes from [10, Definition 4.4], which associates an object $X_{\text {ét }} \in \operatorname{pro}(\mathbb{S})$ to each locally Noetherian simplicial scheme $X$. Finite local systems on $X_{\text {ét }}$ then correspond to finite locally constant étale sheaves on $X$.

Definition 1.20 Given a pro-simplicial set $X$, and a map $\pi_{f} X \rightarrow \Gamma$ to a pro-groupoid with discrete objects, define the covering system $\tilde{X}$ by

$$
\tilde{X}(a):=X \times_{B \Gamma} B(\Gamma \downarrow a) \in \operatorname{pro}(\mathbb{S})
$$

for $a \in \mathrm{Ob} \Gamma$, noting this is equipped with a natural associative action $\Gamma(a, b) \times \tilde{X}(a) \rightarrow$ $\tilde{X}(b)$ in $\operatorname{pro}(\mathbb{S})$. Here, $B$ is the nerve functor (equal to $\bar{W}$ in this context), and $\Gamma \downarrow a$ denotes the slice category of morphisms in $\Gamma$ with target $a$.

Definition 1.21 Given $\pi_{f} X \rightarrow \Gamma$ as above, with a continuous representation $S$ of $\Gamma$ in pro-sets (ie $S(a) \in \operatorname{pro}(\mathrm{Set})$ for $a \in \mathrm{Ob} \Gamma$, equipped with an associative action $\Gamma(a, b) \times S(a) \rightarrow S(b)$ of pro-sets), define the cosimplicial set $\mathrm{C}^{\bullet}(X, S)$ by

$$
\mathrm{C}^{n}(X, S):=\operatorname{Hom}_{\Gamma, \operatorname{pro}(\mathrm{Set})}\left(\tilde{X}_{n}, S\right) \text {. }
$$


From now on, local systems will be abelian unless stated otherwise.

Definition 1.22 Given $X=\left\{X_{\alpha}\right\} \in \operatorname{pro}(\mathbb{S})$ and a local system $M$ on $X_{\beta}$ define cohomology groups by

$$
\mathrm{H}^{*}(X, M):=\underset{\alpha}{\lim } \mathrm{H}^{*}\left(X_{\alpha}, M\right),
$$

where $\mathrm{H}^{*}\left(X_{\alpha},-\right)$ is cohomology with local coefficients, and we also write $M$ for the pullbacks of $M$ to $X_{\alpha}$ and to $X$. Given $G \in \operatorname{pro}(s \mathrm{Gpd})$, set $\mathrm{H}^{*}(G,-):=\mathrm{H}^{*}(\bar{W} G,-)$.

Note that the cosimplicial complex $\mathrm{C}^{\bullet}(X, M)$ extends [11, Section VI.4] to pro-spaces, and that $\mathrm{H}^{*}(X, M)=\mathrm{H}^{*}\left(\mathrm{C}^{\bullet}(X, M)\right)$, the cohomology groups with local coefficients.

Definition 1.23 Given $X \in \operatorname{pro}(\mathbb{S})$ with $X_{0}$ discrete, and an inverse system $M=$ $\left\{M_{i}\right\}_{i \in \mathbb{N}}$ of local systems on $X$, define the continuous cohomology groups $\mathrm{H}^{*}(X, M)$ as follows. First form the cosimplicial complex $\mathrm{C}^{\bullet}(X, M):=\lim \mathrm{C}^{\bullet}\left(X, M_{i}\right)$, for $\mathrm{C}^{\bullet}$ as in Definition 1.21, then set

$$
\mathrm{H}^{*}(X, M):=\mathrm{H}^{*}\left(\mathrm{C}^{\bullet}(X, M)\right),
$$

noting that this agrees with Definition 1.22 when $M_{i}=M$ for all $i$.

Remark 1.24 Observe that there is a short exact sequence

$$
0 \rightarrow \lim ^{1} \mathrm{H}^{n-1}\left(X, M_{i}\right) \rightarrow \mathrm{H}^{n}(X, M) \rightarrow \underset{\lim }{\longleftarrow} \mathrm{H}^{n}\left(X, M_{i}\right) \rightarrow 0,
$$

so $\mathrm{H}^{n}(X, M) \cong \lim \mathrm{H}^{n}\left(X, M_{i}\right)$ whenever the inverse system $\left\{\mathrm{H}^{n-1}\left(X, M_{i}\right)\right\}_{i}$ satisfies the Mittag-Leffler condition (for instance if the groups are finite).

When working with the étale homotopy type $X_{\text {ét }}$, we will usually apply this construction to $\mathbb{Z}_{\ell}$-local systems $\left\{M_{i}=M / \ell^{i}\right\}_{i}$. In that case, the exact sequence above becomes the comparison between étale cohomology and Jannsen's continuous étale cohomology (see Example 3.18 for details).

Lemma 1.25 Given $X \in \mathbb{S}$ and an inverse system $M=\left\{M_{i}\right\}_{i \in \mathbb{N}}$ of local systems on $X$, there is an isomorphism

$$
\mathrm{H}^{*}\left(X, \lim _{\longleftarrow} M_{i}\right) \cong \mathrm{H}^{*}(X, M)
$$

Proof As in Definition $1.22, \mathrm{H}^{*}\left(X, \underset{\lim }{\longleftarrow} M_{i}\right)$ is cohomology of the complex

$$
\lim _{\leftarrow} \mathrm{C}^{\bullet}\left(X, M_{i}\right)=\mathrm{C}^{\bullet}\left(X, \underset{\leftarrow}{\lim } M_{i}\right)
$$

but $\mathrm{C}^{n}\left(X, \underset{\leftarrow}{\lim } M_{i}\right)=\operatorname{Hom}_{\mathrm{Set}}\left(X_{n}, \underset{\leftarrow}{\lim } M_{i}\right)=\underset{\leftarrow}{\lim } \operatorname{Hom}_{\mathrm{Set}}\left(X_{n}, M_{i}\right)=\mathrm{C}^{n}(X, M)$, as required. 
We will occasionally refer to groups and groupoids as "discrete", to distinguish them from topological (or simplicial) groups and groupoids. As in Remark 1.10, we regard a pro-groupoid as a kind of topological groupoid, so "discrete" will indicate that both simplicial and pro structures are trivial.

Definition 1.26 Given a set $L$ of primes, say that a pro-groupoid $G$ with discrete object set is $(L, n)$-good if for all $G^{\wedge} L$-representations $M$ in abelian $L$-groups, the canonical map

$$
\phi_{M}: \mathrm{H}^{i}\left(G^{\wedge} L, M\right) \rightarrow \mathrm{H}^{i}(G, M)
$$

is an isomorphism for all $i \leq n$ and an inclusion for $i=n+1$. When $L$ is the set of all primes, we say that $G$ is $n$-good. Observe that any inverse system of $(L, n)$-good groupoids is $(L, n)$-good. Say that $G$ is $L-\operatorname{good}$ if it is $(L, n)$-good for all $n$.

Lemma 1.27 Free groups are $L$-good for all $L$.

Proof Let $F=F(X)$ be a free group generated by a set $X$, and let $\Gamma:=F^{\wedge} L$. By the argument of [47, I, Section 2.6, Exercise 1(a)], it suffices to show that $\mathrm{H}^{*}(\Gamma, M) \rightarrow$ $\mathrm{H}^{*}(F, M)$ is surjective for all discrete $\Gamma$-representations $M$ in abelian $L$-groups. Since $F$ is free, $\mathrm{H}^{n}(F, M)=0$ for $n>1$, so it only remains to establish surjectivity for $n=1$.

This amounts to showing that every derivation $\alpha: F \rightarrow M$ factors through $\Gamma$. The derivation gives rise to a map $\beta: F \rightarrow M \rtimes G$, for some finite $L$-torsion quotient $G$ of $F$. Since $M \rtimes G$ is an $L$-group, $\beta$ factors through $\Gamma$.

Examples 1.28 (1) $L$-groups are $L$-good.

(2) If $1 \rightarrow F \rightarrow \Gamma \rightarrow \Pi \rightarrow 1$ is an exact sequence of groups, with $F$ and $\Pi$ $L$-good, $F^{\wedge_{L}} \rightarrow \Gamma^{\wedge_{L}}$ injective, and $\mathrm{H}^{a}(F, M)$ finite for all finite $L$-torsion $\Gamma$-modules, then $\Gamma$ is $L$-good.

(3) All finitely generated nilpotent groups are $L-\operatorname{good}$ for all $L$.

(4) The fundamental group of a compact Riemann surface is $L$-good for all $L$.

Proof (2) This is essentially [47, I, Section 2.6, Exercise 2(c)].

(3) Express $\Gamma$ as a successive extension of finite groups and $\mathbb{Z}$, then apply (2).

(4) Choose a smooth complex projective curve $C$ of genus $g>0$, with $\pi_{1}(C)=\Gamma$. It suffices to show that for all finite $L$-torsion $\Gamma^{\wedge} L$-representations $M$, the map

$$
\mathrm{H}^{*}\left(\Gamma^{\wedge L}, M\right) \rightarrow \mathrm{H}_{\mathrm{et}}^{*}(C, M)
$$

is an isomorphism. 
Letting $\widetilde{C}$ be the universal étale pro- $L$ cover of $C$, this is equivalent (by the Serre spectral sequence) to showing that $\mathrm{H}_{\mathrm{et}}^{*}\left(\tilde{C}, \mathbb{F}_{p}\right)=\mathbb{F}_{p}$ for all $p \in L . \widetilde{C}$ is the inverse limit all finite $L$-covers $C^{\prime} \rightarrow C$, giving

$$
\mathrm{H}_{\mathrm{et}}^{i}\left(\widetilde{C}, \mathbb{F}_{p}\right)={\underset{C^{\prime}}{\lim _{\mathrm{e}}}}_{\mathrm{H}_{\mathrm{e}}^{i}}^{i}\left(C^{\prime}, \mathbb{F}_{p}\right)
$$

which can only be nonzero for $i=0,1,2$.

Note that $\pi_{1}(\tilde{C})=\operatorname{ker}\left(\widehat{\Gamma} \rightarrow \Gamma^{\wedge} L\right)$. Thus the pro- $L$ completion $\pi_{1}(\tilde{C})^{\mathrm{ab}, L}$ of the abelianisation of $\pi_{1}(\widetilde{C})$ must be 0 , or we would have a larger pro- $L$ quotient of $\widehat{\Gamma}$ than $\Gamma^{\wedge} L$. Hence $\mathrm{H}_{\text {et }}^{1}\left(\widetilde{C}, \mathbb{F}_{p}\right)=0$ for all $p \in L$.

We now adapt the proof of Schmidt [45, Proposition 15]. Since any curve $C^{\prime}$ has a cover $C^{\prime \prime}$ of degree $p$, with the map $\mathrm{H}_{\text {et }}^{2}\left(C^{\prime}, \mathbb{F}_{p}\right) \rightarrow \mathrm{H}_{\text {ét }}^{2}\left(C^{\prime \prime}, \mathbb{F}_{p}\right)$ thus being 0 , we deduce that $\mathrm{H}_{\text {ét }}^{2}\left(\widetilde{C}, \mathbb{F}_{p}\right)=0$, which completes the proof.

Proposition 1.29 For any $X \in \mathbb{S}$, the canonical morphism

$$
X \rightarrow \bar{W}\left(G(X)^{\wedge} L\right)
$$

in pro(S) induces an isomorphism $\left(\pi_{f} X\right)^{\wedge_{L}} \rightarrow \pi_{f} \bar{W}\left(G(X)^{\wedge} L\right)$ of pro-groupoids, and has the property that for all finite abelian $\left(\pi_{f} X\right)^{\wedge_{L}}$-representations $M$ in $L$-groups, the canonical map

$$
\mathrm{H}^{*}\left(\bar{W}\left(G(X)^{\wedge} L\right), M\right) \rightarrow \mathrm{H}^{*}(X, M)
$$

is an isomorphism.

Proof The statement about fundamental groupoids is immediate, since completion commutes with taking quotients. Now, observe that

$$
\mathrm{H}^{n}\left(\bar{W}\left(G(X)^{\wedge} L\right), M\right) \cong \mathrm{H}^{n}\left(G(X)^{\wedge} L, M\right),
$$

tautologically from Definition 1.22.

It thus suffices to show that the simplicial groupoid $G(X)$ is $L$-good, in the sense that $\mathrm{H}^{*}(G(X), M) \cong \mathrm{H}^{*}\left(G(X)^{\wedge} L, M\right)$ for all $\pi_{0} G(X)^{\wedge} L$-representations in abelian $L$-groups $M$. This is equivalent to showing that for all $x \in X_{0}$, the simplicial groups $G(X)(x, x)$ are $L$-good. This will follow if the groups $G_{n}(x, x)$ are all $L$-good, because there is a spectral sequence

$$
\mathrm{H}^{q}\left(G_{p}, M\right) \Longrightarrow \mathrm{H}^{p+q}(G, M) .
$$

Since the groups $G_{n}(x, x)$ are all free, this then follows from Lemma 1.27. 
Given a property $P$ of groups, we will say that a groupoid $\Gamma$ locally satisfies $P$ if the groups $\Gamma(x, x)$ satisfy $P$, for all $x \in \mathrm{Ob} \Gamma$.

Definition 1.30 Define $\operatorname{pro}(\mathbb{S})_{\delta}$ to be the full subcategory of pro(S) consisting of pro-spaces $X$ for which $X_{0}$ is discrete (as in Remark 1.10, so $X_{0}$ is a set, not just a pro-set).

Define $\mathbb{S}^{\wedge} L$ to be the full subcategory of pro(S $)_{\delta}$ consisting of spaces $X$ for which the groups $\pi_{n}(X, x)$ are all pro- $L$-groups. If $L$ is the set of all primes, we write $\widehat{\mathbb{S}}:=\mathbb{S}^{\wedge} L$.

Definition 1.31 A morphism $f: X \rightarrow Y$ in $\operatorname{pro}(\mathbb{S})_{\delta}$ is said to be an Artin-Mazur weak equivalence if $\pi_{0} X \rightarrow \pi_{0} Y$ is an isomorphism, and the maps $\pi_{n}(X, x) \rightarrow \pi_{n}(Y, f x)$ are pro-isomorphisms for all $n \geq 1$ and all $x \in X_{0}$.

Define $\operatorname{Ho}\left(\operatorname{pro}(\mathbb{S})_{\delta}\right)$ and $\operatorname{Ho}\left(\mathbb{S}^{\wedge} L\right)$ by formally inverting all Artin-Mazur weak equivalences.

In [19], Isaksen established a model structure on $\operatorname{pro}(\mathbb{S})$ with the right properties for modelling pro-homotopy types. In particular, [19, Corollary 7.5] shows that a morphism in $\operatorname{pro}(\mathbb{S})_{\delta}$ is a weak equivalence in $\operatorname{pro}(\mathbb{S})$ if and only if it is an Artin-Mazur weak equivalence.

Proposition 1.32 Fix $N \in[1, \infty]$, and let $f: X \rightarrow Y$ be a morphism in pro(S $)_{\delta}$ such that $\left(\pi_{f} X\right)^{\wedge} L \rightarrow\left(\pi_{f} Y\right)^{\wedge} L$ is a pro-equivalence of pro-groupoids, with the property that for all abelian $\left(\pi_{f} Y\right)^{\wedge} L_{L}$-representations $M$ in $L$-groups, the map

$$
\mathrm{H}^{n}(f): \mathrm{H}^{n}(Y, M) \rightarrow \mathrm{H}^{n}(X, M)
$$

is an isomorphism for all $n \leq N$ and injective for $n=N+1$. Then for all $Z \in \mathbb{S}^{\wedge} L$ with $\pi_{i} Z=0$ for $i>N$ (resp. $i>N+1$ ), the map

$$
f^{*}: \operatorname{Hom}_{\mathrm{Ho}\left(\operatorname{pro}(\mathbb{S})_{\delta}\right)}(Y, Z) \rightarrow \operatorname{Hom}_{\mathrm{Ho}\left(\operatorname{pro}(\mathbb{S})_{\delta}\right)}(X, Z)
$$

is an isomorphism (resp. an inclusion).

Proof First observe that if $M$ is a $\pi_{f}(Y)^{\wedge} L$-representation in abelian pro- $L$ groups, we can express it as an inverse system $\left\{M_{\alpha}\right\}$ of $\pi_{f}(Y)$-representations in $L$-groups. Then the complex $\mathrm{C}^{\bullet}(Y, M)$ of $M$-cochains is given by

$$
\mathrm{C}^{\bullet}(Y, M) \simeq \mathbf{R} \underset{\alpha}{\lim } \mathrm{C}^{\bullet}\left(Y, M_{\alpha}\right)
$$


This implies that for all such $M$, the map $\mathrm{H}^{n}(f): \mathrm{H}^{n}(Y, M) \rightarrow \mathrm{H}^{n}(X, M)$ is an isomorphism for all $n \leq N$, and injective for $n=N+1$.

Now consider the Moore-Postnikov tower [11, Definition VI.3.4] $P_{n} Z$ of a fibrant replacement for $Z$. The pro-equivalence on $\pi_{f}$ gives the required isomorphism if $Z=P_{1} Z$, and we can proceed by induction.

Assume that we have a homotopy class of maps $X \rightarrow P_{n} Z$, for $n<N$. The obstruction to lifting this to a homotopy class of maps $X \rightarrow P_{n+1} Z$ lies in $\mathrm{H}^{n+2}\left(X, \pi_{n+1} Z\right)$, and if nonempty, the latter homotopy class is a principal $\mathrm{H}^{n+1}\left(X, \pi_{n+1} Z\right)$-space. As $\pi_{n+1} Z$ is a pro- $L$-group, the isomorphism $\mathrm{H}^{n+1}(Y,-) \cong \mathrm{H}^{n+1}(X,-)$ and the inclusion $\mathrm{H}^{n+2}(Y,-) \hookrightarrow \mathrm{H}^{n+2}(X,-)$ (resp. the inclusion $\left.\mathrm{H}^{n+1}(Y,-) \hookrightarrow \mathrm{H}^{n+1}(X,-)\right)$ mean that the pro-homotopy class of lifts $Y \rightarrow P_{n+1} Z$ is similarly determined (resp. embeds into the class of lifts $X \rightarrow P_{n+1} Z$ ), completing the inductive step.

Since the map $Z \rightarrow P_{N} Z$ (resp. $Z \rightarrow P_{N+1} Z$ ) is an Artin-Mazur weak equivalence, this completes the proof for $N<\infty$. In the case $N=\infty$, the analysis above gives an isomorphism

$$
f^{*}: \operatorname{Hom}_{\mathrm{Ho}\left(\operatorname{pro}(\mathbb{S})_{\delta}\right)}\left(Y, \underset{n}{\lim _{n}} P_{n} Z\right) \rightarrow \operatorname{Hom}_{\mathrm{Ho}\left(\operatorname{pro}(\mathbb{S})_{\delta}\right)}\left(X, \lim _{\longleftarrow} P_{n} Z\right) ;
$$

since the canonical map $Z \rightarrow \lim _{\leftarrow} P_{n} Z$ is an Artin-Mazur weak equivalence, this completes the proof.

Corollary 1.33 The inclusion functor $\mathbb{S}^{\wedge} L \rightarrow \operatorname{pro}(\mathbb{S})_{\delta}$ has a homotopy left adjoint, which we denote by $X \rightsquigarrow X^{\wedge} L$. This has the property that for $X \in \mathbb{S}^{\wedge} L, X^{\wedge_{L}} \simeq X$.

Proof Proposition 1.29 and Proposition 1.32 imply that for $X \in \mathbb{S}$, the object $X^{\wedge_{L}}:=$ $\bar{W}\left(G(X)^{\wedge_{L}}\right) \in \mathbb{S}^{\wedge_{L}}$ has the required properties. Given an inverse system $X=\left\{X_{\alpha}\right\}$, set $X^{\wedge L}:=\lim _{\longleftarrow}\left(X_{\alpha}\right)^{\wedge L}$.

Remarks 1.34 Comparing with [10, Theorem 6.4 and Corollary 6.5], we see that this gives a generalisation of Artin and Mazur's pro- $L$ homotopy type [2] to unpointed spaces. Their context for pro-homotopy theory was formulated slightly differently, in terms of $\operatorname{pro}(\operatorname{Ho}(\mathbb{S}))$, which is not very well-behaved. See [19] for details of the comparison.

Since this paper was first written, an alternative pro-finite completion functor has been developed by Quick [42]. However, the category of pro-finite homotopy types in [42] is larger than ours, because for its pro-spaces $X$, the pro-set $\pi_{0} X$ is pro-finite rather than discrete. The pro-finite completion functor thus differs from ours in that it also takes the pro-finite completion of the set $\pi_{0} X$. 
An important feature of [42] is the existence of a model structure for pro-finite spaces, and this raises the question of whether there is a model structure on $\operatorname{pro}\left(s \mathrm{Gpd}^{L}\right)$, and how the respective model structures compare. The most likely solution is that there is a fibrantly cogenerated model structure on $\operatorname{pro}\left(s \mathrm{Gpd}_{\mathcal{F}}^{L}\right)$, where $s \operatorname{Gpd}_{\mathcal{F}}^{L}$ is the full subcategory of $s \mathrm{Gpd}^{L}$ consisting of simplicial groupoids with finite object set. For this model structure, the cogenerating fibrations should be morphisms in $s \mathrm{Gpd}_{\mathcal{F}}^{L}$ which are fibrations in $s \mathrm{Gpd}$, possibly with some additional Artinian condition analogous to [40, Theorem 2.14]. The right adjoint $\operatorname{pro}\left(s \mathrm{Gpd}_{\mathcal{F}}^{L}\right) \rightarrow \operatorname{pro}\left(s \mathrm{Gpd}^{L}\right)$ should then induce a fibrantly cogenerated structure on the latter, while the functor $\bar{W}$ from $\operatorname{pro}\left(s \operatorname{Gpd}_{\mathcal{F}}^{L}\right)$ to simplicial pro-finite sets should be a right Quillen equivalence when $L$ is the set of all primes.

\subsection{Comparing homotopy groups}

We now investigate when we can describe the homotopy groups of $X^{\wedge} L$ in terms of the homotopy groups of $X$.

Lemma 1.35 If $A$ is a finitely generated abelian group, then for $n \geq 2$, completion of the Eilenberg-Mac Lane space is given by $K(A, n)^{\wedge} L=K\left(A^{\wedge} L, n\right)$.

Proof By Proposition 1.32, we need to show that the maps

$$
\mathrm{H}^{*}\left(K\left(A^{\wedge} L, n\right), M\right) \rightarrow \mathrm{H}^{*}(K(A, n), M)
$$

are isomorphisms for all abelian $L$-groups $M$. By considering the spectral sequence associated to a filtration, it suffices to consider only the cases $M=\mathbb{F}_{p}$, for $p \in L$.

If $A=A^{\prime} \times A^{\prime \prime}$, then $K(A, n)=K\left(A^{\prime}, n\right) \times K\left(A^{\prime \prime}, n\right)$, so $\mathrm{H}^{*}\left(K(A, n), \mathbb{F}_{p}\right)=$ $\mathrm{H}^{*}\left(K\left(A^{\prime}, n\right), \mathbb{F}_{p}\right) \otimes \mathrm{H}^{*}\left(K\left(A^{\prime \prime}, n\right), \mathbb{F}_{p}\right)$. The structure theorem for finitely generated abelian groups therefore allows us to assume that $A=\mathbb{Z} / q$, for $q$ a prime power or 0 .

Now, if $q$ is neither zero nor a power of $p$, then $\mathrm{H}^{r}\left(K(A, n), \mathbb{F}_{p}\right)=0$ for $r>0$; since $A^{\wedge} L$ is a quotient of $A$, we also get $\mathrm{H}^{r}\left(K\left(A^{\wedge}, n\right), \mathbb{F}_{p}\right)=0$. If $q=p^{s}$, then $A^{\wedge} L=A$, making isomorphism automatic.

If $q=0$, then $A=\mathbb{Z}, A^{\wedge} L=\prod_{\ell \in L} \mathbb{Z}_{\ell}$, and $\mathrm{H}^{r}\left(K\left(\mathbb{Z}_{\ell}, n\right), \mathbb{F}_{p}\right)=0$ for $r>0$ and $\ell \neq p$. We need to show that

$$
\mathrm{H}^{*}\left(K\left(\mathbb{Z}_{p}, n\right), \mathbb{F}_{p}\right) \rightarrow \mathrm{H}^{*}\left(K(\mathbb{Z}, n), \mathbb{F}_{p}\right)
$$

is an isomorphism, or equivalently that $K(\mathbb{Z}, n)^{\wedge p}=K\left(\mathbb{Z}_{p}, n\right)$. This follows from [43, Theorem 1.5]. 
Proposition 1.36 Take a morphism $f: X \rightarrow Y$ in $\operatorname{pro}(\mathbb{S})_{\delta}$ such that $\left(\pi_{f} X\right)^{\wedge} L \rightarrow$ $\left(\pi_{f} Y\right)^{\wedge}$ is a pro-equivalence of pro-groupoids. Then the following are equivalent:

(1) For all abelian $\left(\pi_{f} Y\right)^{\wedge} L$-representations $M$ in $L$-groups, the map

$$
\mathrm{H}^{n}(f): \mathrm{H}^{n}(Y, M) \rightarrow \mathrm{H}^{n}(X, M)
$$

is an isomorphism for all $n \leq N$ and injective for $n=N+1$.

(2) The map

$$
\pi_{n}(f): \pi_{n}\left(X^{\wedge} L, x\right) \rightarrow \pi_{n}\left(Y^{\wedge} L, f y\right)
$$

is a pro-isomorphism for $n \leq N$ and a pro-surjection for $n=N+1$.

In particular, a pro-groupoid $G$ with discrete object set is $(L, N)$-good if and only if

$$
\pi_{n}\left((B G)^{\wedge L}\right)=0
$$

for all $2 \leq n \leq N$.

Proof The key observation is that we have the isomorphism $\operatorname{Hom}_{\mathrm{Ho}\left(\operatorname{pro}(\mathbb{S})_{\delta}\right)}\left(Y, P_{n} Z\right) \cong$ $\operatorname{Hom}_{\mathrm{Ho}\left(\operatorname{pro}(\mathbb{S})_{\delta}\right)}\left(P_{n} Y, P_{n} Z\right)$, which is deduced from the corresponding result for $\mathbb{S}$. Thus Proposition 1.32 implies that

$$
P_{N}\left(X^{\wedge L}\right) \rightarrow P_{N}\left(Y^{\wedge L}\right)
$$

becomes an isomorphism in $\operatorname{Ho}\left(\operatorname{pro}(\mathbb{S})_{\delta}\right)$, while

$$
P_{N+1}\left(X^{\wedge L}\right) \rightarrow P_{N+1}\left(Y^{\wedge L}\right)
$$

is an epimorphism. Since isomorphisms in $\operatorname{Ho}\left(\operatorname{pro}(\mathbb{S})_{\delta}\right)$ are just Artin-Mazur weak equivalences, this completes the "only if" part.

For the converse, note that the hypothesis is equivalent to saying that the homotopy fibre $F$ of $f^{\wedge_{L}}: X^{\wedge_{L}} \rightarrow Y^{\wedge_{L}}$ is $N$-connected, by looking at the long exact sequence of homotopy groups. Thus $\mathrm{H}^{j}(F, A)=0$ for all $0<j \leq N$ and all abelian $L-$ groups $A$. For any $\pi_{f} Y^{\wedge} L$-representation $M$ in abelian $L$-groups, the Leray spectral sequence

$$
\mathrm{H}^{i}\left(Y^{\wedge_{L}}, \mathrm{H}^{j}(F, M)\right) \Longrightarrow \mathrm{H}^{i+j}\left(X^{\wedge_{L}}, f^{-1} M\right)
$$

forces the maps $\mathrm{H}^{i}\left(Y^{\wedge} L, M\right) \rightarrow \mathrm{H}^{i+j}\left(X^{\wedge} L, M\right)$ to be isomorphisms for $i \leq N$ and injective for $i=N+1$, as required.

The final statement is given by taking $X=B G$ and $Y=B\left(G^{\wedge} L\right)$. 
Lemma 1.37 If $f: X \rightarrow Y$ is a morphism in $\operatorname{pro}(\mathbb{S})_{\delta}$ for which the map

$$
\pi_{n}(f): \pi_{n}(X, x) \rightarrow \pi_{n}(Y, f y)
$$

is a pro-isomorphism for $n \leq N$ and a pro-surjection for $n=N+1$, then the map

$$
\pi_{n}(f): \pi_{n}\left(X^{\wedge} L, x\right) \rightarrow \pi_{n}\left(Y^{\wedge} L, f y\right)
$$

is a pro-isomorphism for $n \leq N$ and a pro-surjection for $n=N+1$.

Proof The proof of Proposition 1.36 adapts to show that for any $\pi_{f} Y$-representation $M$, the maps $\mathrm{H}^{i}(Y, M) \rightarrow \mathrm{H}^{i}(X, M)$ are isomorphisms for $i \leq N$ and injective for $i=N+1$. Thus the hypotheses of Proposition 1.36 are satisfied, giving the required results.

Definition 1.38 Given a group-valued representation $H$ of a groupoid $\Gamma$ (ie a functor from $\Gamma$ to the category of groups), recall from [37, Definition 2.15] that the semidirect product $H \rtimes \Gamma$ is a groupoid with objects $\mathrm{Ob}(H \rtimes \Gamma)=\mathrm{Ob}(\Gamma)$ and has $(H \rtimes \Gamma)(x, y)=$ $H_{x} \rtimes \Gamma(x, y)$.

Proposition 1.39 Fix $X \in \mathbb{S}$. If $\pi_{n}(X, x)$ is finitely generated for all $n \leq N$, and if the image of $\pi_{1}(X, x) \rightarrow \operatorname{Aut}\left(\pi_{n}(X, x) \otimes \mathbb{F}_{p}\right)$ is $L$-torsion for all $n \leq N$, all $p \in L$, and all $x \in X$, then there is an exact sequence

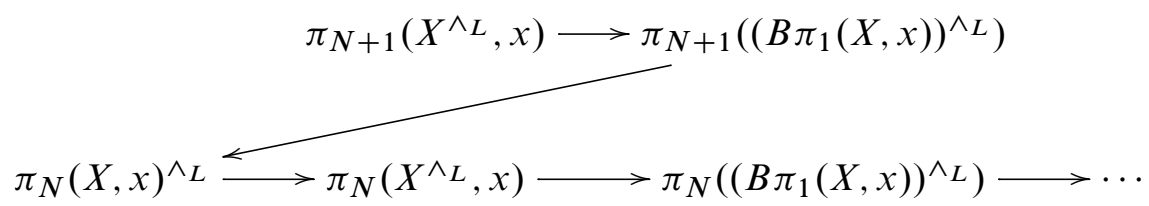

$$
\cdots \longrightarrow \pi_{2}(X, x)^{\wedge_{L}} \longrightarrow \pi_{2}\left(X^{\wedge_{L}}, x\right) \longrightarrow \pi_{2}\left(\left(B \pi_{1}(X, x)\right)^{\wedge_{L}}\right) \longrightarrow 0 .
$$

Hence if in addition $\pi_{f} X$ is $(L, N+1)$-good (resp. $(L, N)$-good), then the natural map

$$
\pi_{n}(X)^{\wedge} L \rightarrow \pi_{n}\left(X^{\wedge} L\right)
$$

is a pro-isomorphism for all $n \leq N$ (resp. a pro-isomorphism for all $n<N$ and a pro-surjection for $n=N$ ).

Proof We adapt the argument of [37, Theorem 1.58]. Let $\{X(n)\}_{n}$ be the Postnikov tower for $X$. We will prove the proposition inductively for the groups $X(n)$. Thanks to Lemma 1.37, we may replace $X$ with $X(N)$, so may assume that the groups $\pi_{n}(X, x)$ are finitely generated for all $n$. Write $\Gamma:=\pi_{f} X$. 
For $n=1, X(1)$ is weakly equivalent to $B \pi_{f} X$, so $\left(B \pi_{f} X\right)^{\wedge L} \simeq X(1)^{\wedge} L$ and $\pi_{n}(X(1), x)=0$ for all $n \geq 2$, making the exact sequence above immediate.

Now assume that $X(n-1)$ satisfies the inductive hypothesis, and consider the fibration $X(n) \rightarrow X(n-1)$. This is determined up to homotopy by a k-invariant $[11$, Section VI.5] $\kappa \in \mathrm{H}^{n+1}\left(X(n-1), \pi_{n}(X)\right)$. Since $\pi_{n}(X) \otimes \mathbb{F}_{p}$ is a finite-dimensional $\Gamma^{\wedge} L$-representation for all $p \in L$, the group $A:=\pi_{n}(X)^{\wedge} L$ is an inverse limit of finite $\Gamma^{\wedge_{L}}$-representations. Now, the element

$$
\kappa \in \mathrm{H}^{n+1}(X(n-1), A) \cong \mathrm{H}^{n+1}\left(X(n-1)^{\wedge} L, A\right)
$$

comes from a map

$$
G(X(n-1))^{\wedge} L \rightarrow\left(N^{-1} A[-n]\right) \rtimes \Gamma,
$$

where $N^{-1}$ denotes the denormalisation functor [52, 8.4.4] from chain complexes to simplicial complexes (the Dold-Kan correspondence).

Let $L A$ be the chain complex with $A$ concentrated in degrees $n, n-1$, and $d:(L A)_{n} \rightarrow$ $(L A)_{n-1}$ the identity, and define $\mathcal{G}$ to be the pullback of this map along the surjection $N^{-1} L A \rtimes \Gamma \rightarrow\left(N^{-1} A[-n]\right) \rtimes \Gamma$ of simplicial locally pro-finite $L$-torsion groupoids. This gives an extension

$$
N^{-1} A[1-n] \rightarrow \mathcal{G} \rightarrow G(X(n-1))^{\wedge L} .
$$

Applying $\bar{W}$ gives the fibration

$$
\bar{W} N^{-1} A[1-n] \rightarrow \bar{W} \mathcal{G} \rightarrow X(n-1)^{\wedge} L
$$

in $\operatorname{pro}(\mathbb{S})$, corresponding to the k-invariant $f^{*} \kappa \in \mathrm{H}^{n}\left(X(n-1)^{\wedge} L, A\right)$ for the map $f: X(n-1) \rightarrow X(n-1)^{\wedge_{L}}$. This in turn gives a map $X(n) \rightarrow \bar{W} \mathcal{G}$, compatible with the fibrations.

The long exact sequence of homotopy applied to the map $\bar{W} \mathcal{G} \rightarrow X(n-1)^{\wedge} L$ shows that $\pi_{m}(\bar{W} \mathcal{G}, x)=\pi_{m}\left(X(n-1)^{\wedge} L\right)$ for all $m \neq n, n+1$, and gives an exact sequence

$$
\begin{aligned}
0 \rightarrow \pi_{n+1}(\bar{W} \mathcal{G}, x) \rightarrow \pi_{n+1}\left(X(n-1)^{\wedge} L\right) & \\
& \rightarrow A(x) \rightarrow \pi_{n}(\bar{W} \mathcal{G}, x) \rightarrow \pi_{n}\left(X(n-1)^{\wedge} L\right) \rightarrow 0 .
\end{aligned}
$$

The inductive hypothesis shows that $\pi_{m}\left(X(n-1)^{\wedge L}\right)=\pi_{m}\left(\left(B \pi_{1}(X, x)\right)^{\wedge} L\right)$ for $m \geq n+1$, so we deduce that there is a long exact sequence

$$
\begin{aligned}
& \cdots \longrightarrow \pi_{m}(X(n), x)^{\wedge_{L}} \longrightarrow \pi_{m}(\bar{W} \mathcal{G}, x) \longrightarrow \pi_{m}\left(\left(B \pi_{1}(X, x)\right)^{\wedge_{L}}\right) \longrightarrow \cdots \\
& \cdots \longrightarrow \pi_{2}(X(n), x)^{\wedge} L \longrightarrow \pi_{2}(\bar{W} \mathcal{G}, x) \longrightarrow \pi_{2}\left(\left(B \pi_{1}(X, x)\right)^{\wedge_{L}}\right) \longrightarrow 0 .
\end{aligned}
$$


As $\bar{W} \mathcal{G} \in \mathbb{S}^{\wedge} L$, it will therefore suffice to show that $F: G(X(n))^{\wedge} L \rightarrow \mathcal{G}$ is a weak equivalence. We now apply the Hochschild-Serre spectral sequence, giving $\mathrm{H}^{p}\left(X(n-1), \mathrm{H}^{q}\left(N^{-1} A[1-n], M\right)\right)=\mathrm{H}^{p}\left(G(X(n-1))^{\wedge} L, \mathrm{H}^{q}\left(N^{-1} A[1-n], M\right)\right)$ $\Longrightarrow \mathrm{H}^{p+q}(\mathcal{G}, M)$.

Similarly $\quad \mathrm{H}^{p}\left(X(n-1), \mathrm{H}^{q}(E(n), V)\right) \Longrightarrow \mathrm{H}^{p+q}(X(n), V)$,

for all $\Gamma^{\wedge} L$-representations $M$ in abelian $L$-groups, where $E(n)$ is the fibre of $X(n) \rightarrow X(n-1)$.

Now, $E(n)$ is a $K\left(\pi_{n}(X), n\right)$-space, and $\bar{W} N^{-1} A[1-n]$ is a $K(A, n)-$ space. By Lemma 1.35 , it follows that $E(n) \rightarrow \bar{W} N^{-1} A[1-n]$ is pro- $L$ completion, giving an isomorphism of cohomology with coefficients in $M$. Thus $F$ induces isomorphisms on homology groups, hence must be a weak equivalence by Proposition 1.32.

Finally, if $\Gamma$ is $(L, m)$-good, Proposition 1.36 shows that $\pi_{n}\left((B \Gamma)^{\wedge}, x\right)=0$ for all $1<n \leq m$.

\section{Review of pro-algebraic homotopy types}

Here we give a summary of the results from $[37 ; 34]$. The motivation for these is that they provide a framework to transfer information about local systems and their cohomology to statements about homotopy types. Fix a field $k$ of characteristic zero.

\subsection{Pro-algebraic groupoids}

Given a local system $\mathbb{V}$ of finite-dimensional $k$-vector spaces on a topological space $X$, we can form the affine $k$-scheme Iso $\left(\mathbb{V}_{x}, \mathbb{V}_{y}\right)$ of isomorphisms of stalks, for each pair of points $x, y \in X$. These combine to form a kind of groupoid $G$ whose objects are the points of $X$. This is the motivating example of a pro-algebraic groupoid; in this case it comes equipped with a canonical groupoid homomorphism $\pi_{f} X \rightarrow G(k)$. For the general case, we now recall some definitions from [37, Sections 2.1-2.3].

Definition 2.1 Define a pro-algebraic groupoid $G$ over a field $k$ to consist of the following data:

(1) A discrete set $\mathrm{Ob}(G)$.

(2) For all $x, y \in \operatorname{Ob}(G)$, an affine scheme $G(x, y)$ (possibly empty) over $k$.

(3) A groupoid structure on $G$, consisting of a multiplication morphism $m: G(x, y) \times$ $G(y, z) \rightarrow G(x, z)$, identities Spec $k \rightarrow G(x, x)$ and inverses $G(x, y) \rightarrow$ $G(y, x)$, satisfying associativity, identity and inverse axioms. 
Note that a pro-algebraic group is just a pro-algebraic groupoid on one object. We say that a pro-algebraic groupoid is reductive (resp. pro-unipotent) if the pro-algebraic groups $G(x, x)$ are so for all $x \in \operatorname{Ob}(G)$. An algebraic groupoid is a pro-algebraic groupoid for which the $G(x, y)$ are all of finite type.

If $G$ is a pro-algebraic groupoid, let $O(G(x, y))$ denote the global sections of the structure sheaf of $G(x, y)$.

Remark 2.2 The terminology "pro-algebraic groupoid" follows the characterisation of pro-algebraic groups in Deligne, Milne, Ogus and Shih [6, Chapter II]. A linear algebraic group is an affine group scheme of finite type, and there is an equivalence of categories between affine group schemes and pro-objects in linear algebraic groups. A more accurate term for pro-algebraic groupoids would thus be "linear pro-algebraically enriched groupoids".

Definition 2.3 Given morphisms $f, g: G \rightarrow H$ of pro-algebraic groupoids, define a natural isomorphism $\eta$ between $f$ and $g$ to consist of morphisms

$$
\eta_{x}: \operatorname{Spec} k \rightarrow H(f(x), g(x))
$$

for all $x \in \operatorname{Ob}(G)$, such that the following diagram commutes, for all $x, y \in \operatorname{Ob}(G)$ :

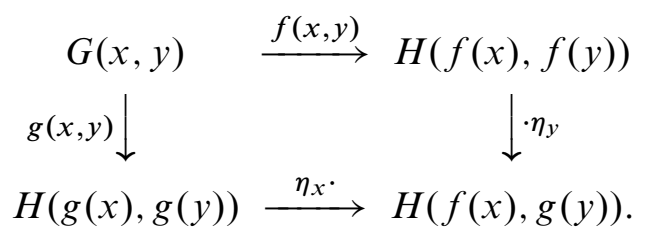

[If we reversed our order of composition in Definition 2.1, this would be the same as a natural transformation of functors of categories enriched in affine $k$-schemes.]

A morphism $f: G \rightarrow H$ of pro-algebraic groupoids is said to be an equivalence if there exists a morphism $g: H \rightarrow G$ such that $f g$ and $g f$ are both naturally isomorphic to identity morphisms. This is the same as saying that for all $y \in \mathrm{Ob}(H)$, there exists $x \in \mathrm{Ob}(G)$ such that $H(f(x), y)(k)$ is nonempty (essential surjectivity), and that for all $x_{1}, x_{2} \in \mathrm{Ob}(G), G\left(x_{1}, x_{2}\right) \rightarrow H\left(f\left(x_{1}\right), f\left(x_{2}\right)\right)$ is an isomorphism.

Definition 2.4 Given a pro-algebraic groupoid $G$, define a finite-dimensional linear $G$-representation to be a functor $\rho$ from $G$ to the category of finite-dimensional $k$-vector spaces, respecting the algebraic structure. Explicitly, this consists of a set $\left\{V_{x}\right\}_{x \in \mathrm{Ob}(G)}$ of finite-dimensional $k$-vector spaces, together with morphisms $\rho_{x y}: G(x, y) \rightarrow \operatorname{Hom}\left(V_{y}, V_{x}\right)$ of affine schemes, respecting the multiplication and identities. 
A morphism $f:(V, \rho) \rightarrow(W, \varrho)$ of $G$-representations consists of $f_{x} \in \operatorname{Hom}\left(V_{x}, W_{x}\right)$ such that

$$
f_{x} \circ \varrho_{x y}=\rho_{x y} \circ f_{y}: G(x, y) \rightarrow \operatorname{Hom}\left(V_{x}, W_{y}\right) .
$$

Definition 2.5 Given a pro-algebraic groupoid $G$, define the reductive quotient $G^{\text {red }}$ of $G$ by setting $\mathrm{Ob}\left(G^{\text {red }}\right)=\operatorname{Ob}(G)$, and

$$
G^{\mathrm{red}}(x, y)=G(x, y) / \mathrm{R}_{\mathrm{u}}(G(y, y))=\mathrm{R}_{\mathrm{u}}(G(x, x)) \backslash G(x, y),
$$

where $\mathrm{R}_{\mathrm{u}}(G(x, x))$ is the pro-unipotent radical of the pro-algebraic group $G(x, x)$. The equality arises since if $f \in G(x, y), g \in \mathrm{R}_{\mathrm{u}}(G(y, y))$, then $f g f^{-1} \in \mathrm{R}_{\mathrm{u}}(G(x, x))$, so both equivalence relations are the same. Multiplication and inversion descend similarly. Observe that $G^{\text {red }}$ is then a reductive pro-algebraic groupoid. Representations of $G^{\text {red }}$ correspond to semisimple representations of $G$, since $k$ is of characteristic 0 .

Definition 2.6 Recall from [6, Definition II.1.7] that a tensor category $\mathcal{C}$ is said to be rigid if it has an internal Hom-functor $\mathcal{H o m}$, satisfying

- $\mathcal{H o m}(X, Y) \otimes \mathcal{H o m}\left(X^{\prime}, Y^{\prime}\right) \cong \mathcal{H o m}\left(X \otimes X^{\prime}, Y \otimes Y^{\prime}\right)$ and

- $\left(X^{\vee}\right)^{\vee} \cong X$ for all $X \in \mathcal{C}$,

where $X^{\vee}=\mathcal{H} \operatorname{Hom}(X, 1)$, with 1 the unit for $\otimes$.

Definition 2.7 Recall from [6, Section II.2] that a neutral Tannakian category over $k$ is a $k$-linear rigid abelian tensor category $\mathcal{C}$, equipped with a faithful exact tensor functor $\omega$ (the fibre functor) from $\mathcal{C}$ to the category of finite-dimensional $k$-vector spaces.

In [37, Section 2.1], this was extended to multifibred Tannakian categories, which have several exact tensor functors $\left\{\omega_{x}\right\}_{x \in S}$, jointly faithful in the sense that $\operatorname{Hom}(U, V) \hookrightarrow$ $\prod_{x \in S} \operatorname{Hom}\left(\omega_{x} U, \omega_{x} V\right)$.

A Tannakian subcategory $\mathcal{D} \subset \mathcal{C}$ is a full subcategory closed under the formation of subquotients, direct sums, tensor products, and duals.

Tannakian duality [6, Theorem II.2.11] then states that for any neutral Tannakian category $(\mathcal{C}, \omega)$ over a field, there is a canonical equivalence between $\mathcal{C}$ and the category of finite-dimensional representations of a unique affine group scheme $G$. Explicitly, $G$ is the scheme of tensor automorphisms of $\omega$.

If $\mathcal{C}$ is multifibred, with a set $S$ of fibre functors, we form a pro-algebraic groupoid $G$ on objects $S$ by setting $G(x, y)$ to be the affine scheme of tensor isomorphisms from $\omega_{x}$ to $\omega_{y}$. This gives a canonical equivalence between $\mathcal{C}$ and the category of finite-dimensional $G$-representations, with $\omega_{x}$ being pullback along the inclusion $\{x\} \hookrightarrow G$. 
Definition 2.8 Let AGpd denote the category of pro-algebraic groupoids over $k$, and observe that this category contains all limits.

Lemma 2.9 Consider the functor $G \mapsto G(k)$ from AGpd to Gpd, the category of abstract groupoids. This has a left adjoint, the algebraisation functor, denoted $\Gamma \mapsto \Gamma^{\mathrm{alg}}$, which is determined by the finite-dimensional linear representations of $\Gamma$.

Proof The algebraisation functor can be given explicitly by setting $\mathrm{Ob}(\Gamma)^{\mathrm{alg}}=\mathrm{Ob}(\Gamma)$, and

$$
\Gamma^{\mathrm{alg}}(x, y)=\Gamma(x, x)^{\mathrm{alg}} \times^{\Gamma(x, x)} \Gamma(x, y),
$$

where $\Gamma(x, x)^{\text {alg }}$ is the pro-algebraic (or Hochschild-Mostow) completion of the group $\Gamma(x, x)$ [17], and $X \times{ }^{G} Y$ is the quotient of $X \times Y$ by the relation $(g x, y) \sim(x, g y)$ for $g \in G$.

Alternatively, the finite-dimensional linear representations of $\Gamma$ (as in Definition 2.4) correspond to those of $\Gamma^{\mathrm{alg}}$ (if the latter exists). These form a multifibred Tannakian category (with one fibre functor for each object of $\Gamma$ ), so Tannakian duality provides unique pro-algebraic groupoid $G$ with the same finite-dimensional representations as $\Gamma$. For any pro-algebraic groupoid $H$ and any groupoid homomorphism $\Gamma \rightarrow H(k)$, we then have a functor from $H$-representations to $\Gamma$ representations, and thus a unique compatible morphism $G \rightarrow H$, so $\Gamma^{\mathrm{alg}} \cong G$.

Example 2.10 The motivating example for this setup is when $\Gamma=\pi_{f} X$, the fundamental groupoid of a topological space. Then $\left(\pi_{f} X\right)^{\text {alg }}$ is the pro-algebraic groupoid corresponding to the multifibred Tannakian category of local systems of finite-dimensional $k$-vector spaces on $X$. The fibre functors are given by $\mathbb{V} \mapsto \mathbb{V}_{x}$. Likewise, $\left(\pi_{f} X\right)^{\text {red }}$ is the object corresponding to the Tannakian category of semisimple local systems.

Definition 2.11 Given a pro-algebraic groupoid $G$, and $U=\left\{U_{x}\right\}_{x \in \mathrm{Ob}(G)}$ a collection of pro-algebraic groups parametrised by $\mathrm{Ob}(G)$, we say that $G$ acts on $U$ if there are morphisms $U_{x} \times G(x, y) \stackrel{*}{\rightarrow} U_{y}$ of affine schemes, satisfying the following conditions:

(1) $(u v) * g=(u * g)(v * g), 1 * g=1$ and $\left(u^{-1}\right) * g=(u * g)^{-1}$, for $g \in G(x, y)$ and $u, v \in U_{x}$.

(2) $u *(g h)=(u * g) * h$ and $u * 1=u$, for $g \in G(x, y), h \in G(y, z)$ and $u \in U_{x}$.

If $G$ acts on $U$, we construct $G \ltimes U$ as in Definition 1.38. 
Definition 2.12 Given a pro-algebraic groupoid $G$, define the pro-unipotent radical $\mathrm{R}_{\mathrm{u}}(G)$ to be the collection $\mathrm{R}_{\mathrm{u}}(G)_{x}=\mathrm{R}_{\mathrm{u}}(G(x, x))$ of pro-unipotent pro-algebraic groups, for $x \in \operatorname{Ob}(G)$. $G$ then acts on $\mathrm{R}_{\mathrm{u}}(G)$ by conjugation, ie

$$
u * g:=g^{-1} u g
$$

for $u \in \mathrm{R}_{\mathrm{u}}(G)_{x}, g \in G(x, y)$.

Now assume that the field $k$ is of characteristic 0 .

Proposition 2.13 For any pro-algebraic groupoid $G$, there is a Levi decomposition $G=G^{\mathrm{red}} \ltimes \mathrm{R}_{\mathrm{u}}(G)$, unique up to conjugation by $\mathrm{R}_{\mathrm{u}}(G)$.

Proof [37, Proposition 2.17].

\subsection{The pro-algebraic homotopy type of a topological space}

We now recall the results from [37, Section 2.4]. The motivation here is that we wish to study the whole homotopy type, not just fundamental groupoids. This will involve working with the loop groupoid, which is a simplicial groupoid, so we need a simplicial framework.

Definition 2.14 Given a simplicial object $G_{\bullet}$ in the category of pro-algebraic groupoids, with $\operatorname{Ob}\left(G_{\bullet}\right)$ constant, define the fundamental groupoid $\pi_{0}\left(G_{\bullet}\right)$ of $G_{\bullet}$ to have objects $\operatorname{Ob}(G)$, and for $x, y \in \operatorname{Ob}(G)$, set $\pi_{0}(G)(x, y)$ to be the coequaliser

$$
G_{1}(x, y) \underset{\partial_{0}}{\stackrel{\partial_{1}}{\longrightarrow}} G_{0}(x, y) \longrightarrow \pi_{0}(G)(x, y)
$$

in the category of affine schemes. Thus $\pi_{0}(G)$ is a pro-algebraic groupoid on objects $\operatorname{Ob}(G)$, with multiplication inherited from $G_{0}$.

Definition 2.15 Define a pro-algebraic simplicial groupoid to consist of a simplicial complex $G \bullet$ of pro-algebraic groupoids, such that

(1) $\mathrm{Ob}\left(G_{\bullet}\right)$ is constant, and

(2) for all $x \in \mathrm{Ob}(G), G(x, x) \bullet \in s \mathrm{AGp}$, ie the maps $G_{n}(x, x) \rightarrow \pi_{0}(G)(x, x)$ are pro-unipotent extensions of pro-algebraic groups.

We denote the category of pro-algebraic simplicial groupoids by $s$ AGpd. 
For any $G_{\bullet} \in s$ AGpd and $x \in \operatorname{Ob}\left(G_{\bullet}\right)$, observe that $G_{\bullet}(x, x)$ is a simplicial affine group scheme, so has homotopy groups $\pi_{n}(G \bullet(x, x))$. That these are also affine group schemes follows from the standard characterisation

$$
\pi_{n}\left(G_{\bullet}(x, x)\right)=\mathrm{H}_{n}\left(N_{\bullet}(x, x), \partial_{0}\right)
$$

of homotopy groups of simplicial groups.

Lemma 2.16 There is a model structure on $s$ AGpd in which a morphism $f: G_{\bullet} \rightarrow H_{\bullet}$ is

(1) a weak equivalence if the map $\pi_{0}(f): \pi_{0}\left(G_{\bullet}\right) \rightarrow \pi_{0}\left(H_{\bullet}\right)$ is an equivalence of proalgebraic groupoids, and the maps $\pi_{n}(f, x): \pi_{n}\left(G_{\bullet}(x, x)\right) \rightarrow \pi_{n}\left(H_{\bullet}(f x, f x)\right)$ are isomorphisms for all $n$ and for all $x \in \mathrm{Ob}(G)$;

(2) a fibration if the morphism $N_{n}(f): N(G(x, x))_{n} \rightarrow N(H(x, x))_{n}$ of normalised groups is surjective for all $n>0$ and all $x \in \mathrm{Ob}(G)$, and $f$ satisfies the pathlifting condition that for all $x \in \mathrm{Ob}(G), y \in \mathrm{Ob}(H)$, and $h \in H_{0}(f x, y)(k)$, there exists $z \in \mathrm{Ob}(G), g \in G_{0}(x, z)(k)$ with $f g=h$. Equivalently, this says that $G(k) \rightarrow H(k)$ is a fibration in the category of simplicial groupoids.

Proof This is [37, Theorem 2.25].

We define $\mathrm{Ho}(s \mathrm{AGpd})$ to be the localisation of $s \mathrm{AGpd}$ at weak equivalences.

There is a forgetful functor $(k): s \mathrm{AGpd} \rightarrow s \mathrm{Gpd}$, given by sending $G_{\bullet}$ to $G_{\bullet}(k)$. This functor has a left adjoint $G_{\bullet} \mapsto\left(G_{\bullet}\right)^{\text {alg }}$. We can describe $\left(G_{\bullet}\right)^{\text {alg }}$ explicitly. First let $\left(\pi_{0}(G)\right)^{\text {alg }}$ be the pro-algebraic completion of the abstract groupoid $\pi_{0}(G)$, then let $\left(G^{\text {alg }}\right)_{n}$ be the relative Malcev completion (defined in [13] for pro-algebraic groups) of the morphism

$$
G_{n} \rightarrow\left(\pi_{0}(G)\right)^{\mathrm{alg}} \text {. }
$$

In other words, $G_{n} \rightarrow\left(G^{\text {alg }}\right)_{n} \stackrel{f}{\rightarrow}\left(\pi_{0}(G)\right)^{\text {alg }}$ is the universal diagram with $f$ a prounipotent extension.

Proposition 2.17 The functors $(k)$ and $(-)^{\text {alg }}$ give rise to a pair of adjoint functors

$$
\mathrm{Ho}(s \mathrm{Gpd}) \underset{(k)}{\stackrel{\mathbb{L}^{\mathrm{alg}}}{\perp}} \mathrm{Ho}(s \mathrm{AGpd}),
$$

with $\mathbb{L}^{\text {alg }} G(X)=G(X)^{\text {alg }}$, for any $X \in \mathbb{S}$ and $G$ as in Definition 1.6.

Proof [37, Proposition 2.26] shows that the functors are a Quillen pair, so the statement follows from the observation that all objects in $s$ AGpd are fibrant, making $(k)$ its own derived right Quillen functor. Since $G(X)$ is cofibrant, $\mathbb{L}^{\text {alg }} G(X)=G(X)^{\text {alg }}$. 
The reason that we need to take $\mathbb{L}^{\text {alg }}$ in the Proposition is that $(-)^{\text {alg }}$ is not an exact functor, so only preserves weak equivalences between cofibrant objects (which roughly correspond to free simplicial groupoids). In Examples 2.24, we will see examples of discrete groups $\Gamma$ for which the map $\mathbb{L}^{\text {alg }} \Gamma \rightarrow \Gamma^{\text {alg }}$ is not a weak equivalence.

Definition 2.18 Given a simplicial set (or equivalently a topological space), define the pro-algebraic homotopy type of $X$ over $k$ to be the object

$$
G(X)^{\text {alg }}
$$

in $\operatorname{Ho}(s \mathrm{AGpd})$, where $G(X)$ is the loop groupoid of Definition 1.6. Define the proalgebraic fundamental groupoid by $\varpi_{f}(X):=\pi_{0}\left(G(X)^{\mathrm{alg}}\right)$. Note that $\pi_{0}\left(G^{\mathrm{alg}}\right)$ is the pro-algebraic completion of the fundamental groupoid $\pi_{0}(G)$.

We then define the higher pro-algebraic homotopy groups $\varpi_{n}(X)$ (as $\varpi_{f} X$-representations) by

$$
\varpi_{n}(X):=\pi_{n-1}\left(G(X)^{\mathrm{alg}}\right),
$$

where $\pi_{n}(G)$ is the representation $x \mapsto \pi_{n}(G(x, x))$, for $x \in \mathrm{Ob}(G)$.

Remark 2.19 We can interpret $G(X)^{\text {alg }}$ as the classifying object for nonabelian cohomology. Given $G \in s \mathrm{AGpd}$, we can define $\mathrm{H}^{1}(X, G)$ to be the homotopy class of maps $G(X)^{\text {alg }} \rightarrow G$, which is just $[X, \bar{W} G(k)]$. When $G$ is just a linear algebraic group, this recovers the usual definition of the set $\mathrm{H}^{1}(X, G)$ of classes of $G$-torsors on $X$. When $A$ is a simplicial finite-dimensional vector space (regarded as a simplicial algebraic group), this definition gives

$$
\mathrm{H}^{1}(X, A)=\mathbb{H}^{1}(X, N A),
$$

hypercohomology of the normalised complex associated to $A$.

\subsection{Relative Malcev homotopy types}

Definition 2.20 Assume we have an abstract groupoid $G$, a reductive pro-algebraic groupoid $R$, and a representation $\rho: G \rightarrow R(k)$ which is an isomorphism on objects and Zariski-dense on morphisms (ie $\rho: G(x, y) \rightarrow R(k)(\rho x, \rho y)$ is Zariski-dense for all $x, y \in \mathrm{Ob} G)$. Define the Malcev completion $(G, \rho)^{\mathrm{Mal}}\left(\right.$ or $G^{\rho, \mathrm{Mal}}$, or $G^{R, \mathrm{Mal}}$ ) of $G$ relative to $\rho$ to be the universal diagram

$$
G \rightarrow(G, \rho)^{\mathrm{Mal}} \stackrel{p}{\rightarrow} R
$$

with $p$ a pro-unipotent extension, and the composition equal to $\rho$. Explicitly, the objects are $\mathrm{Ob}(G, \rho)^{\mathrm{Mal}}=\mathrm{Ob} G$ and

$$
(G, \rho)^{\mathrm{Mal}}(x, y)=(G(x, x), \rho)^{\mathrm{Mal}} \times{ }^{G(x, x)} G(x, y) .
$$


If $G$ and $R$ are groups, observe that this agrees with the usual definition (of [13]).

If $\varrho: G \rightarrow R(k)$ is any Zariski-dense representation (ie essentially surjective on objects and Zariski-dense on morphisms) to a reductive pro-algebraic groupoid (in most examples, we take $R$ to be a group), we can define another reductive groupoid $\widetilde{R}$ by setting $\mathrm{Ob} \widetilde{R}=\mathrm{Ob} G$, and $\widetilde{R}(x, y)=R(\varrho x, \varrho y)$. This gives a representation $\rho: \pi_{f} X \rightarrow \widetilde{R}$ satisfying the above hypotheses, and we define the Malcev completion of $G$ relative to $\varrho$ to be the Malcev completion of $G$ relative to $\rho$. Note that $\widetilde{R} \rightarrow R$ is an equivalence of pro-algebraic groupoids.

Definition 2.21 Given a Zariski-dense morphism $\rho: \pi_{f} X \rightarrow R(k)$, let the Malcev completion $G(X, \rho)^{\mathrm{Mal}}$ of $X$ relative to $\rho$ be the pro-algebraic simplicial group $(G(X), \rho)^{\mathrm{Mal}}$. Observe that the Malcev completion of $X$ relative to $\left(\pi_{f} X\right)^{\text {red }}$ is just $G(X)^{\mathrm{alg}}$. Let $\varpi_{f}(X, \rho)^{\mathrm{Mal}}=\pi_{0} G(X, \rho)^{\mathrm{Mal}}$ and $\varpi_{n}(X, \rho)^{\mathrm{Mal}}=\pi_{n-1} G(X, \rho)^{\mathrm{Mal}}$. Note that $\pi_{f}\left((X, \rho)^{\mathrm{Mal}}\right)$ is the relative Malcev completion of $\rho: \pi_{f} X \rightarrow R(k)$.

Beware that the relative Malcev completion of $X$ is defined by completing a loop space for $X$, rather than $X$ itself. However, Theorem 2.74 will give other equivalent formulations of the homotopy type, effectively by completing a covering space for $X$.

Lemma 2.22 Let $f: X \rightarrow Y$ be a morphism in $\mathbb{S}$ for which the map

$$
\pi_{n}(f): \pi_{n}(X) \rightarrow \pi_{n}(Y)
$$

is an isomorphism for $n \leq N$ and a surjection for $n=N+1$, and take a Zariski-dense morphism $\rho: \pi_{f} Y \rightarrow R(k)$. Then the map

$$
\varpi_{n}(f): \varpi_{n}(X, \rho \circ f)^{\mathrm{Mal}} \rightarrow \varpi_{n}(Y, \rho)^{\mathrm{Mal}}
$$

is an isomorphism for $n \leq N$ and a surjection for $n=N+1$.

Proof As in the proof of Lemma 1.37, for any $\pi_{f} Y$-representation $M$, the maps $\mathrm{H}^{i}(Y, M) \rightarrow \mathrm{H}^{i}(X, M)$ are isomorphisms for $i \leq N$ and injective for $i=N+1$.

Now, [37, Proposition 4.37] gives a convergent Adams spectral sequence

$$
E_{p q}^{1}(X)=\left(\operatorname{Lie}_{-p}\left(\tilde{\mathrm{H}}^{*+1}(X, \mathbb{O}(R))^{\vee}\right)\right)_{p+q} \Longrightarrow \varpi_{p+q+1}(X, \rho \circ f)^{\mathrm{Mal}},
$$

in the category of pro-finite-dimensional vector spaces, where $\tilde{\mathrm{H}}$ denotes reduced cohomology, $\mathrm{Lie}_{*}$ is the free graded Lie algebra functor, and $\mathbb{O}(R)$ is the local system of Definition 2.75. Since $E_{p q}^{1}(X) \rightarrow E_{p q}^{1}(Y)$ is an isomorphism for $p+q<N$ and surjective for $p+q=N$, the result follows. 
Definition 2.23 Say that a groupoid $\Gamma$ is $n$-good with respect to a Zariski-dense representation $\rho: \Gamma \rightarrow R(k)$ to a reductive pro-algebraic groupoid if for all finitedimensional $\Gamma^{\rho, \text { Mal }}$-representations $V$, the map

$$
\mathrm{H}^{i}\left(\Gamma^{\rho, \mathrm{Mal}}, V\right) \rightarrow \mathrm{H}^{i}(\Gamma, V)
$$

is an isomorphism for all $i \leq n$ and an inclusion for $i=n+1$. Say that $\Gamma$ is $\operatorname{good}$ with respect to $\rho$ if it is $n-\operatorname{good}$ for all $n$.

See Lemma 3.36 for alternative criteria to determine when a groupoid is $n$-good.

Examples 2.24 By [37, Examples 3.20], finite groups, free groups, finitely generated nilpotent groups and fundamental groups of compact Riemann surfaces are all good with respect to all Zariski-dense representations. Superrigid groups (such as $\mathrm{SL}_{3}(\mathbb{Z})$ ) give examples of groups which are not good with respect to any real (or complex) representations. This is because $\Gamma^{R, \mathrm{Mal}}=R$ in these cases, but $\mathrm{H}^{*}(\Gamma, \mathbb{R}) \neq \mathbb{R}$.

Theorem 2.25 If $X$ is a topological space with fundamental groupoid $\Gamma$, equipped with a Zariski-dense representation $\rho: \Gamma \rightarrow R(k)$ to a reductive pro-algebraic groupoid for which

(1) $\Gamma$ is $(N+1)$-good with respect to $\rho$,

(2) $\pi_{n}(X,-)$ is of finite rank for all $1<n \leq N$, and

(3) the $\Gamma$-representation $\pi_{n}(X,-) \otimes_{\mathbb{Z}} k$ is an extension of $R$-representations (ie a $\Gamma^{\rho, \text { Mal }}$-representation) for all $1<n \leq N$,

then the canonical map

$$
\pi_{n}(X,-) \otimes_{\mathbb{Z}} k \rightarrow \varpi_{n}\left(X^{\rho, \mathrm{Mal}},-\right)
$$

is an isomorphism for all $1<n \leq N$.

Proof When $N=\infty$, this is [37, Theorem 3.21], but the same proof gives the conclusion above if we only assume that $\Gamma$ is $(N+1)-\operatorname{good}$ (while still requiring the other conditions to hold for all $n$ ). For arbitrary $N$, and $X$ as above, this means that the $N$-th stage $X(N)$ in the Postnikov tower for $X$ gives isomorphisms

$$
\pi_{n}(X,-) \otimes_{\mathbb{Z}} k \rightarrow \varpi_{n}\left(X(N)^{\rho, \mathrm{Mal}},-\right)
$$

for all $1<n \leq N$, since $\pi_{i} X(N)=0$ for $i>N$, while $\pi_{i} X(N)=\pi_{i} X$ for $i \leq N$. Applying Lemma 2.22 to the morphism $X \rightarrow X(N)$ now completes the proof. 


\subsection{Cohomology and hypercohomology}

\subsubsection{Simplicial groupoids}

Definition 2.26 For a simplicial groupoid $\Gamma_{\bullet}$, a cosimplicial $\Gamma_{\bullet}-$ representation consists of the following:

(1) a $\Gamma_{n}$-representation $V^{n}$ for all $n$, with $g \cdot \partial^{i} v=\partial^{i}\left(\left(\partial_{i} g\right) \cdot v\right)$, for $g \in \Gamma_{n+1}$, $v \in V^{n}$

(2) operations $\partial^{i}, \sigma^{i}$ making $V^{\bullet}(x)$ into a cosimplicial complex for each $x \in \mathrm{Ob} \Gamma_{\bullet}$, satisfying the additional conditions that

$$
g \cdot\left(\partial^{i} v\right)=\partial^{i}\left(\left(\partial_{i} g\right) \cdot v\right) \quad h \cdot\left(\sigma^{i} v\right)=\sigma^{i}\left(\left(\sigma_{i} g\right) \cdot v\right)
$$

for $g \in \Gamma_{n+1}(x, y), h \in \Gamma_{n-1}(x, y), v \in V^{n}(y)$.

Remark 2.27 If $\Gamma_{\bullet}=G(X)$, then we can think of a cosimplicial $\Gamma_{\bullet}-$ representation as being a kind of hyperlocal system on $X$. As we will see below, these give a sufficiently large category to recover cohomology, but objects with constant cosimplicial structure are still just local systems.

Definition 2.28 Given a simplicial groupoid $\Gamma_{\bullet}$ and a cosimplicial $\Gamma_{\bullet}-$ representation $V$, define the cosimplicial complex $\mathrm{C}^{\bullet}\left(\Gamma_{\bullet}, V\right)$ by

$$
\mathrm{C}^{n}\left(\Gamma_{\bullet}, V\right)=\operatorname{Hom}_{\Gamma_{n}}\left(\left(W \Gamma_{\bullet}\right)_{n}, V^{n}\right),
$$

for the functor $W$ from Definition 1.5, with operations $\left(\partial^{i} f\right)(x)=\partial_{V}^{i}\left(f\left(\partial_{i} x\right)\right)$ for $x \in\left(W \Gamma_{\bullet}\right)_{n+1}$, and $\left(\sigma^{i} f\right)(x)=\sigma_{V}^{i}\left(f\left(\sigma_{i} x\right)\right)$ for $x \in\left(W \Gamma_{\bullet}\right)_{n-1}$.

Then define hypercohomology groups $\mathbb{H}^{i}\left(\Gamma_{\bullet}, V\right)$ by $\mathbb{H}^{i}\left(\Gamma_{\bullet}, V\right)=\mathrm{H}^{i} \mathrm{C}\left(\Gamma_{\bullet}, V\right)$. If $V$ is a $\pi_{0} \Gamma_{\bullet}$-representation, regard $V$ as a cosimplicial $\Gamma_{\bullet}$-representation (with constant cosimplicial structure) and write $\mathrm{H}^{i}\left(\Gamma_{\bullet}, V\right):=\mathbb{H}^{i}\left(\Gamma_{\bullet}, V\right)$.

Lemma 2.29 If $\Gamma_{\bullet}$ is a simplicial groupoid and $V$ a $\pi_{0} \Gamma_{\bullet}$-representation, then

$$
\mathrm{H}^{i}\left(\Gamma_{\bullet}, V\right)=\mathrm{H}^{i}\left(\bar{W} \Gamma_{\bullet}, V\right) .
$$

Proof Observe that $\pi_{0}\left(\Gamma_{\bullet}\right) \times \Gamma_{\bullet}\left(W \Gamma_{\bullet}\right)$ is the universal covering system of $\bar{W} \Gamma_{\bullet}$. Since $V$ is a $\pi_{0} \Gamma_{\bullet}$-representation,

$$
\begin{aligned}
\operatorname{Hom}_{\Gamma_{n}}\left(\left(W \Gamma_{\bullet}\right)_{n}, V\right) & =\operatorname{Hom}_{\pi_{0} \Gamma_{\bullet}}\left(\left(\pi_{0} \Gamma_{\bullet}\right) \times \Gamma^{\Gamma_{n}}\left(W \Gamma_{\bullet}\right)_{n}, V\right) \\
& =\operatorname{Hom}_{\pi_{f} \bar{W} \Gamma}\left(\left(\overline{\left.\bar{W} \Gamma_{\bullet}\right)_{n}}, V\right),\right.
\end{aligned}
$$

so $\mathrm{C}^{\bullet}\left(\Gamma_{\bullet}, V\right)=\mathrm{C}^{\bullet}\left(\bar{W} \Gamma_{\bullet}, V\right)$ (as defined in Definition 1.21), which gives the result. 
Lemma 2.30 If $\Gamma_{\bullet}$ is a simplicial groupoid and $V$ a cosimplicial $\Gamma_{\bullet}-$ representation, then there is a convergent spectral sequence

$$
\mathrm{H}^{i}\left(\Gamma_{\bullet}, \mathrm{H}^{j}(V)\right) \Longrightarrow \mathbb{H}^{i+j}\left(\Gamma_{\bullet}, V\right),
$$

where $H^{j}(V)$ is the $\pi_{0} \Gamma_{\bullet}$-representation given by setting $\mathrm{H}^{*}(V)(x)$ to be cohomology of the cosimplicial complex $V(x)$, for all $x \in \mathrm{Ob} \Gamma$ •.

Proof Form the filtration $\left\{F_{n} V\right\}_{n}$ of $V$ by setting $F_{n} V$ to be the image of the $n-$ skeleton $\operatorname{sk}^{n} V \rightarrow V ; F_{n} V$ is the subcomplex of $V$ generated under the operations $\partial^{i}$ by $V^{\leq n}$, and its Dold-Kan normalisation is given by

$$
N\left(F_{n} V\right)^{i}= \begin{cases}N^{i} V & i \leq n, \\ d N^{n} V & i=n+1, \\ 0 & i \geq n+2 .\end{cases}
$$

Note that the condition $g \partial^{i} v=\partial^{i}\left(\left(\partial_{i} g\right) v\right.$ implies that $F_{n} V$ is $\Gamma_{\bullet}-$ equivariant. Also note that $F_{n} V / F_{n-1} V$ is quasi-isomorphic to the denormalisation $D H^{n}(V)[-n]$. The spectral sequence associated to this filtration is thus

$$
\mathbb{H}^{i+j}\left(\Gamma_{\bullet}, D \mathrm{H}^{j}(V)[-j]\right) \Longrightarrow \mathbb{H}^{i+j}\left(\Gamma_{\bullet}, V\right) .
$$

Let $K_{\bullet}:=\operatorname{ker}\left(\Gamma_{\bullet} \rightarrow \pi_{0} \Gamma_{\bullet}\right)$; since $\mathrm{H}^{j} V$ is a $\pi_{0} \Gamma_{\bullet}$-representation, there is a bicosimplicial complex

with

$$
C^{a, b}:=\operatorname{Hom}_{\pi_{0}\left(\Gamma_{\bullet}\right)}\left(K_{a} \backslash\left(W \Gamma_{\bullet}\right)_{a}, D^{b} \mathrm{H}^{j}(V)[-j]\right),
$$

By the Eilenberg-Zilber Theorem [52, Theorem 8.5.1], $N \operatorname{diag} C$ is quasi-isomorphic to the total complex of $\operatorname{Hom}_{\pi_{0} \Gamma_{\bullet}}\left(N \mathbb{Z}\left(K_{\bullet} \backslash W \Gamma_{\bullet}\right), \mathrm{H}^{j}(V)[-j]\right)$, therefore $\mathrm{H}^{n}(C)=$ $\mathrm{H}^{n-j}\left(G, \mathrm{H}^{j}(V)\right)$, and the spectral sequence becomes

$$
\mathbb{H}^{i}\left(\Gamma_{\bullet}, H^{j}(V)\right) \Longrightarrow \mathbb{H}^{i+j}\left(\Gamma_{\bullet}, V\right) .
$$

Lemma 2.31 Given a weak equivalence $f: \Gamma_{\bullet} \rightarrow \Delta_{\bullet}$ of simplicial groupoids, and a cosimplicial $\Gamma_{\bullet}-$ representation $V$, the map

$$
f^{*}: \mathbb{H}^{*}\left(\Delta_{\bullet}, V\right) \rightarrow \mathbb{H}^{*}\left(\Gamma_{\bullet}, f^{-1} V\right)
$$

is an isomorphism.

Proof Lemma 2.30 gives a morphism of convergent spectral sequences, so we may assume that $V$ is a $\pi_{0} \Delta_{\bullet}-$ representation. Since $\bar{W} f: \bar{W} \Gamma_{\bullet} \rightarrow \bar{W} \Delta \bullet$ is a weak equivalence of simplicial sets, Lemma 2.29 completes the proof. 
Lemma 2.32 Given a simplicial group $\Gamma_{\bullet}$, a cosimplicial $\Gamma_{\bullet}-$ representation $V$ and a simplicial abelian group $A$, the simplicial abelian group

$$
\operatorname{Tot}(V \otimes A)
$$

has a canonical $\Gamma_{\bullet}$-action, where Tot: $\mathbb{S}^{\Delta} \rightarrow \mathbb{S}$ is the total space functor of [11, Chapter VIII], originally defined in [3, Chapter X].

Proof Given $X \in \mathbb{S}^{\Delta}$ and $K \in \mathbb{S}$, define $e(X, K) \in \mathbb{S}^{\Delta}$ by $e(X, K)^{n}:=\left(X^{n}\right)^{K_{n}}$, with obvious cosimplicial operations. Note that $\operatorname{Tot}(e(X, K))=\operatorname{Tot}(X)^{K}$.

The $\Gamma_{\bullet}$-action on $V$ is the same as a cosimplicial map $f: V \rightarrow e\left(V, \Gamma_{\bullet}\right)$, so we have maps

$$
V \otimes A \stackrel{f}{\rightarrow} e\left(V, \Gamma_{\bullet}\right) \otimes A \rightarrow e\left(V \otimes A, \Gamma_{\bullet}\right),
$$

thus a map $\operatorname{Tot}(V \otimes A) \rightarrow \operatorname{Tot}(V \otimes A)^{\Gamma}$. This is equivalent to a map $\Gamma_{\bullet} \times \operatorname{Tot}(V \otimes A) \rightarrow$ $\operatorname{Tot}(V \otimes A)$ of simplicial sets, and the argument above adapts to show that this action is associative.

In order to simplify the definitions and exposition, we will now take $\Gamma_{\bullet}$ to be simplicial group, although everything can be extended to simplicial groupoids.

Definition 2.33 For a simplicial group $\Gamma_{\bullet}$, a simplicial $\Gamma_{\bullet}-$ representation consists of a simplicial abelian group $A$, together with a $\Gamma_{n}$-action on $A_{n}$ for all $n$, compatible with the simplicial operations. Let $s \operatorname{Rep}\left(\Gamma_{\bullet}\right)$ be the category of simplicial $\Gamma \bullet-$ representations.

Note that Lemma 2.32 provides us with examples of simplicial $\Gamma_{\bullet}-$ representations constructed from cosimplicial $\Gamma_{\bullet}-$ representations. Also note that for any simplicial $\Gamma_{\bullet}-$ representation $V$, taking duals levelwise gives a cosimplicial $\Gamma_{\bullet}$-representation $V^{\vee}$ given by $\left(V^{\vee}\right)^{n}=\left(V_{n}\right)^{\vee}$.

Lemma 2.34 Given a simplicial group $\Gamma_{\bullet}$, there is a cofibrantly generated model structure on $s \operatorname{Rep}\left(\Gamma_{\bullet}\right)$, in which a morphism $f: A \rightarrow B$ is

(1) a weak equivalence if the maps $\pi_{i}(f): \pi_{i}(A) \rightarrow \pi_{i}(B)$ are isomorphisms for all $i$;

(2) a fibration if the underlying map in $\mathbb{S}$ is a fibration, or equivalently if the maps $N_{i}(f): N_{i}(A) \rightarrow N_{i}(B)$ on the Dold-Kan normalisation are surjective for all $i>0$. 
Proof The forgetful functor from $s \operatorname{Rep}\left(\Gamma_{\bullet}\right)$ to simplicial sets preserves filtered direct limits and has a left adjoint $F(S)=\mathbb{Z}\left(\Gamma_{\bullet} \times S\right)$. Thus for any finite object $I \in \mathbb{S}$, the object $F I$ is finite in $s \operatorname{Rep}\left(\Gamma_{\bullet}\right)$, so a fortiori permits the small object argument. The model structure on $\mathbb{S}$ is cofibrantly generated by finite objects, so [16, Theorem 11.3.2] gives the required model structure on $s \operatorname{Rep}\left(\Gamma_{\bullet}\right)$.

Lemma 2.35 We may characterise hypercohomology groups by

$$
\mathbb{H}^{i}\left(\Gamma_{\bullet}, V\right)=\operatorname{Hom}_{\mathrm{Ho}\left(s \mathrm{Ab}\left(\Gamma_{\bullet}\right)\right)}\left(\mathbb{Z}, \operatorname{Tot}\left(V \otimes_{\mathbb{Z}} N^{-1} \mathbb{Z}[-i]\right)\right) .
$$

Proof We first note that $\mathbb{Z}\left[W \Gamma_{\bullet}\right]$ is a cofibrant replacement for $\mathbb{Z}$, so for a simplicial abelian group $A$,

$$
\mathbf{R} \underline{\operatorname{Hom}}_{s \mathrm{Ab}\left(\Gamma_{\bullet}\right)}\left(\mathbb{Z}, \operatorname{Tot}\left(V \otimes_{\mathbb{Z}} A\right)\right) \simeq \underline{\operatorname{Hom}}_{s \mathrm{Ab}\left(\Gamma_{\bullet}\right)}\left(\mathbb{Z}\left[W \Gamma_{\bullet}\right], \operatorname{Tot}\left(V \otimes_{\mathbb{Z}} A\right)\right) ;
$$

as observed in the proof of Lemma 2.32,

$$
\underline{\operatorname{Hom}}_{s \mathrm{Ab}}\left(\mathbb{Z}\left[W \Gamma_{\bullet}\right], \operatorname{Tot}\left(V \otimes_{\mathbb{Z}} A\right)\right) \cong \operatorname{Tot}\left(e\left(V \otimes_{\mathbb{Z}} A, W \Gamma_{\bullet}\right)\right),
$$

so

$$
\underline{\operatorname{Hom}}_{s \mathrm{Ab}\left(\Gamma_{\bullet}\right)}\left(\mathbb{Z}\left[W \Gamma_{\bullet}\right], \operatorname{Tot}\left(V \otimes_{\mathbb{Z}} A\right)\right) \cong \operatorname{Tot}\left(e\left(V \otimes_{\mathbb{Z}} A, W \Gamma_{\bullet}\right)^{\Gamma_{\bullet}}\right) .
$$

Now, $e\left(V \otimes_{\mathbb{Z}} A, W \Gamma_{\bullet}\right)^{\Gamma_{\bullet}}$ is given in simplicial level $n$ by $\mathrm{C}^{\bullet}\left(\Gamma_{\bullet}, V \otimes_{\mathbb{Z}} A_{n}\right)$. When $A_{n}$ is free and finitely generated, this becomes $\mathrm{C}^{\bullet}\left(\Gamma_{\bullet}, V\right) \otimes_{\mathbb{Z}} A_{n}$. Taking $A=N^{-1} \mathbb{Z}[-i]$ thus gives

$$
\begin{aligned}
\operatorname{Hom}_{\mathrm{Ho}\left(s \mathrm{Ab}\left(\Gamma_{\bullet}\right)\right)}(\mathbb{Z}, & \left.\operatorname{Tot}\left(V \otimes_{\mathbb{Z}} N^{-1} \mathbb{Z}[-i]\right)\right) \\
& \cong \pi_{0} \mathbf{R} \underline{\operatorname{Hom}}_{s \mathrm{Ab}\left(\Gamma_{\bullet}\right)}\left(\mathbb{Z}, \operatorname{Tot}\left(V \otimes_{\mathbb{Z}} N^{-1} \mathbb{Z}[-i]\right)\right) \\
& \cong \operatorname{Tot}\left(\mathrm{C}^{\bullet}\left(\Gamma_{\bullet}, V\right) \otimes_{\mathbb{Z}} N^{-1} \mathbb{Z}[-i]\right) .
\end{aligned}
$$

Given a cosimplicial simplicial abelian group $B$, the normalisation $N$ Tot $B$ is equivalent to the good truncation in nonnegative chain degrees of the product total complex $\operatorname{Tot}^{\Pi} N_{c} N B$ of the binormalisation of $B$ (which is a cochain chain complex). Thus

$$
\pi_{0} \operatorname{Tot}\left(\mathrm{C}^{\bullet}\left(\Gamma_{\bullet}, V\right) \otimes_{\mathbb{Z}} N^{-1} \mathbb{Z}[-i]\right) \cong \mathrm{H}_{0} \operatorname{Tot}^{\Pi}\left(\left(N_{c} \mathrm{C}^{\bullet}\left(\Gamma_{\bullet}, V\right)\right) \otimes_{\mathbb{Z}} \mathbb{Z}[-i]\right),
$$

and $\operatorname{Tot}^{\Pi}\left(\left(N_{c} \mathrm{C}^{\bullet}\left(\Gamma_{\bullet}, V\right)\right) \otimes_{\mathbb{Z}} \mathbb{Z}[-i]\right)$ is just the complex $N_{c} \mathrm{C}^{\bullet}\left(\Gamma_{\bullet}, V\right)$ turned upside down and shifted $i$ places, so

$$
\mathrm{H}_{0} \operatorname{Tot}^{\Pi}\left(\left(N_{c} \mathrm{C}^{\bullet}\left(\Gamma_{\bullet}, V\right)\right) \otimes_{\mathbb{Z}} \mathbb{Z}[-i]\right)=\mathrm{H}^{i} N_{c} \mathrm{C}^{\bullet}\left(\Gamma_{\bullet}, V\right)=\mathbb{H}^{i}\left(\Gamma_{\bullet}, V\right),
$$

as required.

The following is an analogue of the Leray spectral sequence, and will play a key role in Theorem 3.32. 
Proposition 2.36 Given a surjection $\Gamma_{\bullet} \rightarrow \Delta \bullet$ of simplicial groups with kernel $B_{\bullet}$, and a cosimplicial $\Gamma_{\bullet}$-representation $V$, there is a canonical convergent spectral sequence

$$
\mathrm{H}^{i}\left(\Delta_{\bullet}, \mathbb{H}^{j}\left(B_{\bullet}, V\right)\right) \Longrightarrow \mathbb{H}^{i+j}\left(\Gamma_{\bullet}, V\right),
$$

which we refer to as the Hochschild-Serre spectral sequence.

Proof Given $T \in s \operatorname{Ab}\left(\Delta_{\bullet}\right)$ and $U, W \in s \operatorname{Ab}\left(\Gamma_{\bullet}\right)$, we have an isomorphism

$$
\underline{\operatorname{Hom}}_{s \mathrm{Ab}\left(\Delta_{\bullet}\right)}\left(T, \underline{\operatorname{Hom}}_{s \mathrm{Ab}\left(B_{\bullet}\right)}(U, W)\right) \cong \underline{\operatorname{Hom}}_{s \mathrm{Ab}\left(\Gamma_{\bullet}\right)}(T \otimes U, W) .
$$

This defines a right Quillen functor $s \mathrm{Ab}\left(\Delta_{\bullet}\right)^{\mathrm{opp}} \times s \mathrm{Ab}\left(\Gamma_{\bullet}\right)^{\mathrm{opp}} \times s \mathrm{Ab}\left(\Gamma_{\bullet}\right) \rightarrow \mathbb{S}$; since any cofibrant $\Gamma_{\bullet}-$ representation is cofibrant as a $B_{\bullet}$-representation, the isomorphism above gives an equivalence

$$
\mathbf{R} \operatorname{Hom}_{s \mathrm{Ab}\left(\Delta_{\bullet}\right)}\left(T, \mathbf{R} \underline{\operatorname{Hom}}_{s \mathrm{Ab}\left(B_{\bullet}\right)}(U, W)\right) \simeq \mathbf{R} \underline{\operatorname{Hom}}_{s \mathrm{Ab}\left(\Gamma_{\bullet}\right)}\left(T \otimes \otimes^{\mathbf{L}} U, W\right) .
$$

In particular,

$$
\mathbf{R} \underline{\operatorname{Hom}}_{s \mathrm{Ab}\left(\Gamma_{\bullet}\right)}(\mathbb{Z}, W) \simeq \mathbf{R} \operatorname{Hom}_{s \mathrm{Ab}\left(\Delta_{\bullet}\right)}\left(\mathbb{Z}, \mathbf{R} \underline{\operatorname{Hom}}_{s \mathrm{Ab}\left(B_{\bullet}\right)}(\mathbb{Z}, W)\right) .
$$

Setting $W=\operatorname{Tot}\left(V \otimes_{\mathbb{Z}} N^{-1} \mathbb{Z}[-n]\right)$, this gives an isomorphism

$$
\mathbb{H}^{n}\left(\Gamma_{\bullet}, V\right) \cong \mathrm{H}^{n} \mathrm{C}^{\bullet}\left(\Delta_{\bullet}, \mathrm{C}^{\bullet}\left(B_{\bullet}, V\right)\right),
$$

so the morphism $\mathrm{C}^{\bullet}\left(\Gamma_{\bullet}, V\right) \rightarrow \mathrm{C}^{\bullet}\left(\Delta_{\bullet}, \mathrm{C}^{\bullet}\left(B_{\bullet}, V\right)\right)$ is a quasi-isomorphism, and the result now follows from Lemma 2.30 .

\subsubsection{Simplicial pro-algebraic groupoids}

Definition 2.37 Given $G \in s$ AGpd, define a cosimplicial $G$-representation to be an $O(G)$-comodule $V$ in cosimplicial $k$-vector spaces. Thus we have cosimplicial complexes $V(x)$ for all $x \in \mathrm{Ob} G$, together with a coassociative coaction $V(x) \rightarrow$ $O(G)(x, y) \otimes V(y)$.

Note that the category of cosimplicial $G$-representations is opposite to the category $s \widehat{\operatorname{FDRep}}(G)$ of pro-finite-dimensional simplicial $G$-representations from [37, Section 1.5].

Definition 2.38 Given $G \in s$ AGpd and a cosimplicial $G$-representation $V$, define the cosimplicial complex $\mathrm{C}^{\bullet}(G, V)$ by

$$
\mathrm{C}^{n}(G, V)=O\left((W G)_{n}\right) \otimes{ }^{G_{n}} V^{n},
$$

for the functor $W$ from Definition 1.5, with operations $\partial^{i} \otimes \partial^{i}$ and $\sigma^{i} \otimes \sigma^{i}$. 
Then define hypercohomology groups $\mathbb{H}^{i}(G, V)$ by $\mathbb{H}^{i}(G, V)=\mathrm{H}^{i} \mathrm{C}(G, V)$. If $V$ is a $\pi_{0} G$-representation, regard $V$ as a cosimplicial $G$-representation (with constant cosimplicial structure) and write $\mathrm{H}^{i}(G, V):=\mathbb{H}^{i}(G, V)$.

Now, [37, Example 1.45] ensures that $\mathbb{H}^{i}(G, V)^{\vee}=\mathrm{H}_{i}\left(G, V^{\vee}\right)$ in the notation of [37, Definition 1.48]. In particular, this means that hypercohomology groups of $G$ are an invariant of the homotopy type of $G$.

Proposition 2.39 A morphism $f: G \rightarrow K$ of pro-algebraic simplicial groupoids is a weak equivalence if and only if

(1) $f\left(\mathrm{R}_{\mathrm{u}}(G)\right) \leq \mathrm{R}_{\mathrm{u}}(K)$, with the quotient map

$$
G^{\mathrm{red}} \rightarrow K^{\mathrm{red}}
$$

an equivalence, and

(2) for all finite-dimensional irreducible $K$-representations $V$, the maps

$$
\mathrm{H}^{i}(f): \mathrm{H}^{i}(K, V) \rightarrow \mathrm{H}^{i}\left(G, f^{*} V\right)
$$

are isomorphisms for all $i>0$.

Proof This is [37, Corollary 1.55], adapted from groups to groupoids.

Note that the analogue of Lemma 2.32 for pro-algebraic simplicial groupoids thus ensures that weak equivalences induce isomorphisms on hypercohomology.

Lemma 2.40 For a cofibrant pro-algebraic simplicial group $G$ (for the model structure of Lemma 2.16), and a finite-dimensional $\pi_{0} G$-representation $V$, the cohomology group $\mathrm{H}^{i}(G, V)$ is isomorphic to the homotopy class of maps $G \rightarrow G \ltimes\left(N^{-1} V[1-i]\right)$ in the model category $s \operatorname{AGpd} \downarrow G$.

Proof Consider the morphism $k \rightarrow O(G)$, and let the cokernel be $C$. As in the proof of [37, Proposition 1.50], $C$ is fibrant as a cosimplicial $G$-representation. Likewise, $V \otimes O(G)$ and $V \otimes C$ are both fibrant, so $\mathrm{H}^{*}(G, V)$ is cohomology of the cone complex of

$$
V \otimes^{G} O(G) \rightarrow V \otimes \otimes^{G} C .
$$

Now, $V \otimes G(G)$ is just $V$, so we need to describe $V \otimes \otimes^{G} C$.

Letting $E:=O(G)^{\vee}$, we see that $C^{\vee}$ is the kernel of $E \rightarrow k$. Elements $\theta$ of $V \otimes_{n}^{G} C^{n}$ are then just morphisms $\theta:\left(C^{\vee}\right)_{n} \rightarrow V$ satisfying $\alpha(g c)=g \alpha(c)$, for $g \in G_{n}, c \in\left(C^{\vee}\right)_{n}$. There is a map $E \rightarrow C^{\vee}$ given by $a \mapsto a-1$, so $\theta$ composed 
with this gives a linear morphism $\theta^{\prime}: E_{n} \rightarrow V$, satisfying $\theta^{\prime}(g a)=g \theta^{\prime}(a)+\theta^{\prime}(g)$ for $g \in G_{n}$.

Regarding $E_{n}$ as an affine scheme, there is a morphism $G_{n} \rightarrow E_{n}$, so we see that $\theta$ corresponds to a derivation $\theta^{\prime}: G_{n} \rightarrow V$. Since derivations $G \rightarrow V$ are just morphisms $G \rightarrow G \ltimes V$ over $G$, the statement now follows from the description of the path object in $s$ AGpd from [37, Lemma 2.29].

Lemma 2.41 If $\Gamma_{\bullet}$ is a cofibrant simplicial groupoid (eg $G(X)$ for $X \in \mathbb{S}$ ), and $V$ is a finite-dimensional $\pi_{0} \Gamma_{\bullet}^{R, \mathrm{Mal}}$-representation, then the map

$$
\mathrm{H}^{*}\left(\Gamma_{\bullet}^{R, \mathrm{Mal}}, V\right) \rightarrow \mathrm{H}^{*}\left(\Gamma_{\bullet}, V\right)
$$

is an isomorphism.

Proof This is implicit in [37, Section 1.5.3]. Replacing $\Gamma_{\bullet}$ with a disjoint union of simplicial groups, Lemma 2.40 gives that $\mathrm{H}^{*}\left(\Gamma_{\bullet}^{R, \mathrm{Mal}}, V\right)$ is the homotopy class of maps from $\Gamma_{\bullet}^{R \text {,Mal }}$ to $\Gamma_{\bullet}^{R, \text { Mal }} \ltimes\left(N^{-1} V[1-i]\right)$ over $\Gamma_{\bullet}^{R, \text { Mal }}$. Since any map from $\Gamma_{\bullet}^{\text {alg }}$ to a pro-unipotent extension of $R$ factors through $\Gamma_{\bullet}^{R, \mathrm{Mal}}$, this is the same as the homotopy class of maps from $\Gamma_{\bullet}^{\text {alg }}$ to $\Gamma_{\bullet}^{R, \mathrm{Mal}} \ltimes\left(N^{-1} V[1-i]\right)$ over $\Gamma_{\bullet}^{R, \mathrm{Mal}}$.

The Quillen adjunction of Proposition 2.17 then shows that this is equivalent to the homotopy class of maps from $\Gamma_{\bullet}$ to $\Gamma_{\bullet} \ltimes\left(N^{-1} V[1-i]\right)$ in the slice category $s G p d \downarrow \Gamma_{\bullet}$, which is just $\mathrm{H}^{i}\left(\Gamma_{\bullet}, V\right)$.

Note if we have $\Gamma_{\bullet} \in s$ Gpd and $G \in s$ AGpd together with a morphism $f: \Gamma_{\bullet} \rightarrow G(k)$ of simplicial groupoids, then every cosimplicial $G$-representation $V$ naturally gives rise to a cosimplicial $\Gamma_{\bullet}-$ representation $f^{*} V$. For any coalgebra $C$, every $C-$ comodule is a nested union of finite-dimensional comodules. Thus every cosimplicial $G$-representation $V$ is a filtered direct limit $\lim _{\rightarrow \alpha} V_{\alpha}$ of levelwise finite-dimensional cosimplicial $G$-representations, and we tweak the construction of pullbacks slightly by regarding $f^{*} V$ as the ind-object (ie filtered direct system) $\left\{f^{*} V_{\alpha}\right\}$ of levelwise finite-dimensional cosimplicial $\Gamma_{\bullet}-$ representations. We then define $\mathrm{C}^{\bullet}\left(\Gamma_{\bullet}, f^{*} V\right):=$ $\lim _{\rightarrow \alpha} \mathrm{C}^{\bullet}\left(\Gamma_{\bullet}, f^{*} V_{\alpha}\right)$, and $\mathbb{H}^{*}\left(\Gamma_{\bullet}, f^{*} V\right):=\mathrm{H}^{*} \mathrm{C}^{\bullet}\left(\Gamma_{\bullet}, f^{*} V\right)=\lim _{\rightarrow \alpha} \mathbb{H}^{*}\left(\Gamma_{\bullet}, f^{*} V_{\alpha}\right)$.

Also note that the category of cosimplicial $G$-representations is opposite to the category $s \widehat{\operatorname{FDRep}}(G)$ of [37, Section 1.5].

Lemma 2.42 Given a cofibrant simplicial groupoid $\Gamma_{\bullet}$ and a cosimplicial $O\left(\Gamma_{\bullet}^{R, \mathrm{Mal}}\right)-$ comodule $V$, the canonical map

$$
\mathbb{H}^{*}\left(\Gamma_{\bullet}^{R, \mathrm{Mal}}, V\right) \rightarrow \mathbb{H}^{*}\left(\Gamma_{\bullet}, V\right)
$$

(induced by the morphism $\left.W \Gamma_{\bullet} \rightarrow W\left(\Gamma_{\bullet}^{R, \mathrm{Mal}}\right)\right)$ is an isomorphism. 
Proof By Lemma 2.30 and its analogue for $s$ AGpd, we have convergent spectral sequences

$$
\begin{aligned}
& \mathbb{H}^{i}\left(\Gamma, H^{j}(V)\right) \Longrightarrow \mathbb{H}^{i+j}\left(\Gamma_{\bullet}, V\right) \\
& \mathbb{H}^{i}\left(G, \mathrm{H}^{j}(V)\right) \Longrightarrow \mathbb{H}^{i+j}(G, V) .
\end{aligned}
$$

For ind-finite-dimensional $\pi_{0} G$-representations $U$, the maps $\mathrm{H}^{i}(G, U) \rightarrow \mathrm{H}^{i}\left(\Gamma_{\bullet}, U\right)$ are isomorphisms by Lemma 2.41, so the maps $\mathrm{H}^{i}\left(G, \mathrm{H}^{j}(V)\right) \rightarrow \mathrm{H}^{i}\left(\Gamma_{\bullet}, \mathrm{H}^{j}(V)\right)$ are isomorphisms, making the morphism of spectral sequences an isomorphism.

Theorem 2.43 Take a fibration $f:(X, x) \rightarrow(Y, y)$ (of pointed connected topological spaces) with connected fibres, and set $F:=f^{-1}(y)$. Take a Zariski-dense representation $\rho: \pi_{1}(X, x) \rightarrow R(k)$ to a reductive pro-algebraic group $R$, let $K$ be the closure of $\rho\left(\pi_{1}(F, x)\right)$, and set $T:=R / K$. If the monodromy action of $\pi_{1}(Y, y)$ on $\mathrm{H}^{*}(F, V)$ factors through $\varpi_{1}(Y, y)^{T, \text { Mal }}$ for all $K$-representations $V$, then $G(F, x)^{K, \text { Mal }}$ is the homotopy fibre of $G(X, x)^{R, \mathrm{Mal}} \rightarrow G(Y, y)^{T, \mathrm{Mal}}$.

Proof This is [34, Theorem 3.10], which uses Lemma 2.42 to show that

$$
\mathrm{H}^{*}\left(G(F, x)^{K, \mathrm{Mal}}, O(K)\right)
$$

and cohomology $\mathrm{H}^{*}(\mathcal{F}, O(K))$ of the homotopy fibre $\mathcal{F}$ are both

$$
\mathbb{H}^{*}\left(G(X, x), O(R) \otimes O(T) O\left(G(Y, y)^{T, \mathrm{Mal}}\right)\right) \cong \mathrm{H}^{j}(F, O(K)) .
$$

\subsection{Equivalent formulations}

Fix a reductive pro-algebraic groupoid $R$.

\subsubsection{Lie algebras}

Definition 2.44 Recall that a Lie coalgebra $C$ is said to be conilpotent if the iterated cobracket $\Delta_{n}: C \rightarrow C^{\otimes n}$ is 0 for sufficiently large $n$. A Lie coalgebra $C$ is indconilpotent if it is a filtered direct limit (or, equivalently, a nested union) of conilpotent Lie coalgebras.

Definition 2.45 Recall from [37, Definition 5.8] that for any $k$-algebra $A$, we define $\hat{\mathcal{N}}_{A}(R)$ to be opposite to the category of $R$-representations in ind-conilpotent Lie coalgebras over $A$, and denote the contravariant equivalence by $C \mapsto C^{\vee}$.

Note that there is a continuous functor $\widehat{\mathcal{N}}_{k}(R) \rightarrow \widehat{\mathcal{N}}_{A}(R)$ given by $C^{\vee} \mapsto\left(C \otimes_{k} A\right)^{\vee}$. We denote this by $\mathfrak{g} \mapsto \mathfrak{g} \hat{\otimes} A$. 
Remark 2.46 Observe that $\mathfrak{g} \in \hat{\mathcal{N}}_{A}(R)$ can be regarded as an object of the category $\operatorname{Aff}_{A}(R)$ of $R$-representations in affine $A$-schemes, by regarding it as the functor

$$
\mathfrak{g}(B):=\operatorname{Hom}_{A, R}\left(\mathfrak{g}^{\vee}, B\right),
$$

for $B \in \operatorname{Alg}_{A}(R):=A \downarrow \operatorname{Alg}(R)$. In fact, $\mathfrak{g}(B)$ is then a Lie algebra over $B$, so the Campbell-Baker-Hausdorff formula defines a group structure on $\mathfrak{g}(B)$, and the resulting group is denoted by $\exp (\mathfrak{g})(B)$. Thus $\exp (\mathfrak{g})$ is an $R$-representation in affine group schemes over $A$ (ie a group object of $\operatorname{Aff}_{A}(R)$ ).

Definition 2.47 Write $s \hat{\mathcal{N}}_{A}(R)$ for the category of simplicial objects in $\hat{\mathcal{N}}_{A}(R)$. A weak equivalence in $s \hat{\mathcal{N}}_{A}(R)$ is a map which gives isomorphisms on cohomology groups of the duals (which are just $A$-modules). We denote by $\operatorname{Ho}\left(s \hat{\mathcal{N}}_{A}(R)\right)$ the localisation of $s \hat{\mathcal{N}}_{A}(R)$ at weak equivalences.

For $k=A$, we will usually drop the subscript, so $\hat{\mathcal{N}}(R):=\widehat{\mathcal{N}}_{k}(R)$, and so on.

Definition 2.48 Define $\mathcal{E}(R)$ to be the full subcategory of $\operatorname{AGpd} \downarrow R$ consisting of those morphisms $\rho: G \rightarrow R$ of pro-algebraic groupoids which are pro-unipotent extensions. Similarly, define $s \mathcal{E}(R)$ to consist of the pro-unipotent extensions in $s \operatorname{AGpd} \downarrow R$, and $\operatorname{Ho}\left(s \mathcal{E}(R)_{*}\right)$ to be full subcategory of $\mathrm{Ho}(\mathrm{Ob} R \downarrow s \mathrm{AGpd})$ on objects $s \mathcal{E}(R)$.

Definition 2.49 Given a pro-algebraic groupoid $R$, define the category $s \mathcal{P}_{A}(R)$ to have the same objects as $s \hat{\mathcal{N}}_{A}(R)$, with morphisms given by

$$
\operatorname{Hom}_{s \mathcal{P}(R)}(\mathfrak{g}, \mathfrak{h})=\exp \left(\prod_{x \in \mathrm{Ob} R} \pi_{0} \mathfrak{h}(x)\right) \times \times^{\exp \left(\mathfrak{h}_{0}^{R}\right)} \operatorname{Hom}_{\mathrm{Ho}(s \hat{\mathcal{N}}(R))}(\mathfrak{g}, \mathfrak{h}),
$$

where $\mathfrak{h}_{0}^{R}$ (the Lie subalgebra of $R$-invariants in $\mathfrak{h}_{0}$ ) acts by conjugation on the set of homomorphisms. Composition of morphisms is given by $(u, f) \circ(v, g)=$ $(u \circ f(v), f \circ g)$.

The following is a key comparison result, which will be used in Proposition 2.76 and Theorem 3.30 as a step towards reformulating Malcev homotopy types in terms of Godement resolutions.

Proposition 2.50 For any reductive pro-algebraic groupoid $R$, the categories $\operatorname{Ho}\left(s \mathcal{E}(R)_{*}\right)$ and $s \mathcal{P}(R)$ are equivalent.

Proof This is part of [37, Theorem 4.41], adapting [37, Proposition 3.15] to the unpointed case. The proof just exploits the Levi decomposition of Proposition 2.13. 
Explicitly, the functor maps $\mathfrak{g} \in s \mathcal{P}(R)$ to the simplicial pro-algebraic group given in level $n$ by $R \ltimes \exp \left(\mathfrak{g}_{n}\right)$. Given a morphism

$$
(u, f) \in \exp \left(\prod_{x \in \mathrm{Ob} R} \pi_{0} \mathfrak{h}(x)\right) \times \times^{\exp \left(\mathfrak{h}^{R}\right)} \operatorname{Hom}_{\mathrm{Ho}(s \mathcal{N}(R))}(\mathfrak{g}, \mathfrak{h}),
$$

lift $u$ to $\tilde{u} \in \prod_{x \in \mathrm{Ob} R} \exp \left(\mathfrak{h}_{0}(x)\right)$, and construct the morphism

$$
\operatorname{ad}_{\tilde{u}} \circ(R \ltimes \exp (f)): R \ltimes \exp (\mathfrak{g}) \rightarrow R \ltimes \exp (\mathfrak{h})
$$

in $s \mathcal{E}(R)$, where for $a \in(R \ltimes \exp (\mathfrak{h}))(x, y)$, we set $\operatorname{ad}_{\tilde{u}}(a)=\widetilde{u}(x) \cdot a \cdot \widetilde{u}(y)^{-1}$.

Definition 2.51 We can now define the multipointed Malcev homotopy type of $X$ relative to $\rho$ to be the image of $G(X, \rho)^{\mathrm{Mal}}$ in $\operatorname{Ho}\left(s \mathcal{E}(\widetilde{R})_{*}\right)$, or equivalently $\mathrm{R}_{\mathrm{u}} G(X, \rho)^{\mathrm{Mal}}$ in $s \mathcal{P}(\widetilde{R})$. Define the unpointed Malcev homotopy type of $X$ relative to $\rho$ to be the image of $G(X, \rho)^{\mathrm{Mal}}$ in $\operatorname{Ho}(s \mathcal{E}(\widetilde{R}))$.

Since $\widetilde{R} \rightarrow R$ is an equivalence of groupoids, there is an equivalence $\operatorname{Ho}(s \mathcal{E}(R)) \rightarrow$ $\operatorname{Ho}(s \mathcal{E}(\widetilde{R})$ ), so may discard some basepoints to give an object of $s \mathcal{P}(R)$ (or equivalently of $\operatorname{Ho}\left(s \mathcal{E}(R)_{*}\right)$ ) whenever $\rho$ is surjective on objects.

\subsubsection{Chain Lie algebras}

Definition 2.52 Let $d g \widehat{\mathcal{N}}_{A}$ be opposite to the category of nonnegatively graded indconilpotent cochain Lie coalgebras over $A$. Define $d g \hat{\mathcal{N}}_{A}(R)$ to be the category of $R$-representations in $d g \hat{\mathcal{N}}_{A}$. For $k=A$, we will usually drop the subscript, so $d g \hat{\mathcal{N}}(R):=d g \hat{\mathcal{N}}_{k}(R)$, and so on.

The following is [37, Lemma 5.9]:

Lemma 2.53 There is a closed model structure on $d g \hat{\mathcal{N}}_{A}(R)$ in which a morphism $f: \mathfrak{g} \rightarrow \mathfrak{h}$ is

(1) a fibration whenever the underlying map $f^{\vee}: \mathfrak{h}^{\vee} \rightarrow \mathfrak{g}^{\vee}$ of cochain complexes over $A$ is injective in strictly positive degrees;

(2) a weak equivalence whenever the maps $\mathrm{H}^{i}\left(f^{\vee}\right): \mathrm{H}^{i}\left(\mathfrak{h}^{\vee}\right) \rightarrow \mathrm{H}^{i}\left(\mathfrak{g}^{\vee}\right)$ are isomorphisms for all $i$.

Remark 2.54 It follows from the construction in [37, Lemma 5.9] that for cofibrant objects $\mathfrak{g} \in d g \hat{\mathcal{N}}(R)$ (taking $A$ to be a field), $\mathfrak{g}^{\vee}$ is freely cogenerated as a graded Lie coalgebra. Thus $\mathfrak{g}^{\vee}[-1]$ is a positively graded strong homotopy commutative algebra without unit (in the sense of [26, Lectures 8 and 15]), and a choice of cogenerators on $\mathfrak{g}^{\vee}$ is the same as a positively graded $E_{\infty}$ (also known as $C_{\infty}$ ) algebra - this is an aspect of Koszul duality. 
Definition 2.55 We say that a morphism $f: \mathfrak{g} \rightarrow \mathfrak{h}$ in $d g \hat{\mathcal{N}}(R)$ is free if there exists a (pro-finite-dimensional) sub- $R$-representation $V \subset \mathfrak{h}$ such that $\mathfrak{h}$ is the free pronilpotent graded Lie algebra over $\mathfrak{g}$ on generators $V$.

Proposition 2.56 (Minimal models) For every object $\mathfrak{g}$ of $d g \hat{\mathcal{N}}(R)$, there exists a free chain Lie algebra $\mathfrak{m}$ with $d=0$ on the abelianisation $\mathfrak{m} /[\mathfrak{m}, \mathfrak{m}]$, unique up to nonunique isomorphism, together with a weak equivalence $\mathfrak{m} \rightarrow \mathfrak{g}$.

Proof [37, Proposition 4.7].

The significance of this result is that, together with Proposition 2.50, it allows us to reformulate Malcev homotopy types in terms of extra structure on cohomology groups, since $(\mathfrak{m} /[\mathfrak{m}, \mathfrak{m}])_{n}$ is dual to $\mathrm{H}^{n+1}(\mathfrak{g}, k)$.

Definition 2.57 Let $d g \mathcal{P}(R)$ be the category with the same objects as $d g \hat{\mathcal{N}}_{A}(R)$, and morphisms given by

$$
\operatorname{Hom}_{d g \mathcal{P}(R)}(\mathfrak{g}, \mathfrak{h})=\exp \left(\prod_{x \in \mathrm{Ob} R} \mathrm{H}_{0} \mathfrak{h}(x)\right) \times x^{\exp \left(\mathfrak{h}{ }_{0}^{R}\right)} \operatorname{Hom}_{\mathrm{Ho}\left(d g \hat{\mathcal{N}}_{A}(R)\right)}(\mathfrak{g}, \mathfrak{h}),
$$

where $\mathfrak{h}_{0}^{R}$ (the Lie subalgebra of $R$-invariants in $\mathfrak{h}_{0}$ ) acts by conjugation on the set of homomorphisms. Composition of morphisms is given by $(u, f) \circ(v, g)=$ $(u \circ f(v), f \circ g)$.

Proposition 2.58 There is a normalisation functor $N: s \widehat{\mathcal{N}}_{A}(R) \rightarrow d g \widehat{\mathcal{N}}_{A}(R)$ such that

$$
\mathrm{H}_{i}(N \mathfrak{g}) \cong \pi_{i}(\mathfrak{g}),
$$

giving equivalences $\operatorname{Ho}\left(s \hat{\mathcal{N}}_{A}(R)\right) \simeq \operatorname{Ho}\left(d g \hat{\mathcal{N}}_{A}(R)\right)$, and $s \mathcal{P}_{A}(R) \simeq d g \mathcal{P}_{A}(R)$.

Proof This is essentially [37, Propositions 4.12 and 5.11], adapted as in [34, Theorem 3.28].

\subsubsection{Cosimplicial algebras}

Definition 2.59 Let $c \operatorname{Alg}(R)$ be the category of $R$-representations in cosimplicial $k$-algebras. 
Proposition 2.60 There is a simplicial model category structure on $c \operatorname{Alg}(R)$, in which a map $f: A \rightarrow B$ is

(1) a weak equivalence if $\mathrm{H}^{i}(f): \mathrm{H}^{i}(A) \rightarrow \mathrm{H}^{i}(B)$ is an isomorphism in $\operatorname{Rep}(R)$ for all $i$;

(2) a fibration if $f^{i}(x): A^{i}(x) \rightarrow B^{i}(x)$ is a surjection for all $x \in \mathrm{Ob}(R)$ and all $i$.

Proof This is [37, Proposition 3.26], adapting [49, Section 2.1].

Definition 2.61 Let $c \operatorname{Alg}(R)_{*}$ be the category of $R$-representations in cosimplicial $k$-algebras, equipped with an augmentation to $\prod_{x \in \mathrm{Ob} R} O(R)(x,-)$. This inherits a model structure from $c \operatorname{Alg}(R)$. Denote the opposite category by $s \operatorname{Aff}(R)_{*}=$ $\bigsqcup_{x \in \mathrm{Ob} R} R(x,-) \downarrow s \operatorname{Aff}(R)$, where the coproduct is taken in the category of affine schemes.

Definition 2.62 Given representations $V, W \in \operatorname{Rep}(R)$, define

$$
V \otimes R \text { } W:=\operatorname{Hom}_{R e p(R)}(k, V \otimes W) .
$$

Definition 2.63 Given $A \in c \operatorname{Alg}(R)$ and $\mathfrak{g} \in s \hat{\mathcal{N}}(R)$, define the Maurer-Cartan space $\operatorname{MC}(A, G)$ to consist of sets $\left\{\omega_{n}\right\}_{n \geq 0}$, with $\omega_{n} \in \exp \left(A^{n+1} \hat{\otimes}^{R} \mathfrak{g}_{n}\right)$, such that

$$
\begin{aligned}
\partial_{i} \omega_{n} & = \begin{cases}\partial^{i+1} \omega_{n-1} & i>0, \\
\left(\partial^{1} \omega_{n-1}\right) \cdot\left(\partial^{0} \omega_{n-1}\right)^{-1} & i=0,\end{cases} \\
\sigma_{i} \omega_{n} & =\sigma^{i+1} \omega_{n+1}, \\
\sigma^{0} \omega_{n} & =1,
\end{aligned}
$$

where $\exp \left(A^{n+1} \hat{\otimes}^{R} \mathfrak{g}_{n}\right)$ is the group with underlying set the Lie algebra $A^{n+1} \hat{\otimes} \mathfrak{g}_{n-1}$, with multiplication given by the Campbell-Baker-Hausdorff formula.

Definition 2.64 Given $A \in c \operatorname{Alg}(R)$ and $\mathfrak{g} \in s \hat{\mathcal{N}}(R)$, define the gauge group $\operatorname{Gg}(A, \mathfrak{g}) \leq \prod_{n} \exp \left(A^{n} \widehat{\otimes}^{R} \mathfrak{g}_{n}\right)$ to consist of those $g$ satisfying

$$
\begin{array}{ll}
\partial_{i} g_{n}=\partial^{i} g_{n-1} & \forall i>0, \\
\sigma_{i} g_{n}=\sigma^{i} g_{n+1} & \forall i .
\end{array}
$$

This has an action on $\operatorname{MC}(A, \mathfrak{g})$ given by

$$
(g * \omega)_{n}=\left(\partial_{0} g_{n+1}\right) \cdot \omega_{n} \cdot\left(\partial^{0} g_{n}^{-1}\right) .
$$


Definition 2.65 Let $c \operatorname{Alg}(R)_{0 *}$ be the full subcategory of $c \operatorname{Alg}(R)_{*}$ whose objects satisfy $\mathrm{H}^{0}(A) \cong k$. Let $\operatorname{Ho}\left(c \operatorname{Alg}(R)_{0 *}\right)$ be the full subcategory of $\operatorname{Ho}\left(c \operatorname{Alg}(R)_{0 *}\right)$ with objects in $c \operatorname{Alg}(R)_{0 *}$. Let $s \operatorname{Aff}(R)_{0 *}$ be the category opposite to $c \operatorname{Alg}(R)_{0 *}$, and $\operatorname{Ho}\left(s \operatorname{Aff}(R)_{0 *}\right)$ opposite to $\operatorname{Ho}\left(c \operatorname{Alg}(R)_{0 *}\right)$.

Definition 2.66 Given a topological space $X$, and a sheaf $\mathscr{F}$ on $X$, define

$$
\mathrm{C}^{n}(X, \mathscr{F}):=\prod_{f:\left|\Delta^{n}\right| \rightarrow X} \Gamma\left(\left|\Delta^{n}\right|, f^{-1} \mathscr{F}\right) .
$$

Together, these form a cosimplicial complex $\mathrm{C}^{\bullet}(X, \mathscr{F})$.

\subsubsection{Cochain algebras}

Definition 2.67 Define $D G \operatorname{Alg}(R)$ to be the category of $R$-representations in nonnegatively graded cochain $k$-algebras, and let $d g \operatorname{Aff}(R)$ be the opposite category.

Lemma 2.68 There is a closed model structure on $D G \operatorname{Alg}(R)$ in which a morphism $f: A \rightarrow B$ is

(1) a weak equivalence if $\mathrm{H}^{i}(f): \mathrm{H}^{i}(A) \rightarrow \mathrm{H}^{i}(B)$ is an isomorphism in $\operatorname{Rep}(R)$ for all $i$;

(2) a fibration if $f^{i}: A^{i} \rightarrow B^{i}$ is a surjection for all $i$;

(3) a cofibration if it has $L L P$ with respect to all trivial fibrations.

Proof This is standard (see eg [23, Proposition 4.1]).

Definition 2.69 Define $D G \operatorname{Alg}(R)_{*}$ to be the category of $R$-representations in nonnegatively graded cochain $k$-algebras, equipped with an augmentation to $\prod_{x \in \mathrm{Ob} R} O(R)(x,-)$. This inherits a model structure from $D G \operatorname{Alg}(R)$. Define $d g \operatorname{Aff}(R)_{*}$ to be the category opposite to $D G \operatorname{Alg}(R)_{*}$.

Let $D G \operatorname{Alg}(R)_{0 *}$ be the full subcategory of $D G \operatorname{Alg}(R)_{*}$ whose objects $A$ satisfy $\mathrm{H}^{0}(A)=k$. Let $\operatorname{Ho}\left(D G \operatorname{Alg}(R)_{*}\right)_{0}$ be the full subcategory of $\operatorname{Ho}\left(D G \operatorname{Alg}(R)_{*}\right)$ on the objects of $D G \operatorname{Alg}(R)_{0}$. Let $d g \operatorname{Aff}(R)_{0 *}$ and $\operatorname{Ho}\left(d g \operatorname{Aff}(R)_{*}\right)_{0}$ be the opposite categories to $D G \operatorname{Alg}(R)_{0 *}$ and $\operatorname{Ho}\left(D G \operatorname{Alg}(R)_{*}\right)_{0}$, respectively.

Proposition 2.70 There is a denormalisation functor $D: D G \operatorname{Alg}(R) \rightarrow c \operatorname{Alg}(R)$ such that

$$
\mathrm{H}^{i}(D A) \cong \mathrm{H}^{i}(A) .
$$

This is a right Quillen equivalence, with left adjoint $D^{*}$, so gives an equivalence $\operatorname{Ho}(c \operatorname{Alg}(R)) \simeq \operatorname{Ho}(D G \operatorname{Alg}(R))$.

Proof This is [37, Proposition 4.27]. 
Definition 2.71 Given a cochain algebra $A \in D G \operatorname{Alg}(R)$, and a chain Lie algebra $\mathfrak{g} \in d g \hat{\mathcal{N}}(R)$, define the Maurer-Cartan space by

$$
\operatorname{MC}(A, \mathfrak{g}):=\left\{\omega \in \bigoplus_{n} A^{n+1} \hat{\otimes}^{R} \mathfrak{g}_{n} \mid d \omega+\frac{1}{2}[\omega, \omega]=0\right\} .
$$

Definition 2.72 Given $A \in D G \operatorname{Alg}(R)$ and $\mathfrak{g} \in d g \hat{\mathcal{N}}(R)$, we define the gauge group by

$$
\operatorname{Gg}(A, \mathfrak{g}):=\exp \left(\prod_{n} A^{n} \hat{\otimes}^{R} \mathfrak{g}_{n}\right) .
$$

Define a gauge action of $\operatorname{Gg}(A, \mathfrak{g})$ on $\operatorname{MC}(A, \mathfrak{g})$ by

$$
g(\omega):=g \cdot \omega \cdot g^{-1}-(d g) \cdot g^{-1} .
$$

Definition 2.73 Recall that the Thom-Sullivan (or Thom-Whitney) functor Th from cosimplicial algebras to DG algebras is defined as follows. Let $\Omega\left(\left|\Delta^{n}\right|\right)$ be the DG algebra of rational polynomial forms on the $n$-simplex, so

$$
\Omega\left(\left|\Delta^{n}\right|\right)=\mathbb{Q}\left[t_{0}, \ldots, t_{n}, d t_{0}, \ldots, d t_{n}\right] /\left(1-\sum_{i} t_{i}, \sum_{i} d t_{i}\right),
$$

where $t_{i}$ is of degree 0 . The usual face and degeneracy maps for simplices yield $\partial_{i}: \Omega\left(\left|\Delta^{n}\right|\right) \rightarrow \Omega\left(\left|\Delta^{n-1}\right|\right)$ and $\sigma_{i}: \Omega\left(\left|\Delta^{n}\right|\right) \rightarrow \Omega\left(\left|\Delta^{n-1}\right|\right)$, giving a simplicial complex of DGAs. Given a cosimplicial algebra $A$, we then set

$$
\operatorname{Th}(A):=\left\{a \in \prod_{n} A^{n} \otimes \Omega\left(\left|\Delta^{n}\right|\right): \partial_{A}^{i} a_{n}=\partial_{i} a_{n+1}, \sigma_{A}^{j} a_{n}=\sigma_{j} a_{n-1} \forall i, j\right\} .
$$

The following is a major comparison result, which will be used in Theorem 3.30 as the main step towards reformulating Malcev homotopy types in terms of Godement resolutions.

Theorem 2.74 We have the following commutative diagram of equivalences of categories:

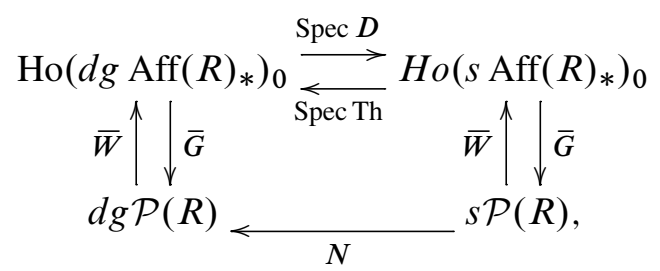


with the pair

$$
\operatorname{Ho}\left(d g \operatorname{Aff}(R)_{*}\right)_{0} \underset{\bar{W}}{\stackrel{\bar{G}}{\rightleftarrows}} d g \mathcal{P}(R),
$$

characterised by the property that

$$
\begin{aligned}
\operatorname{Hom}_{\mathrm{Ho}\left(d g \operatorname{Aff}(R)_{*}\right)}(\operatorname{Spec} A, \bar{W} \mathfrak{g}) & =\operatorname{Hom}_{d g \mathcal{P}(R)}(\bar{G}(A), \mathfrak{g}) \\
& =\operatorname{MC}(A, \mathfrak{g}) \times^{\operatorname{Gg}(A, \mathfrak{g})} \prod_{x \in \mathrm{Ob} R} \exp \left(\mathrm{H}_{0} \mathfrak{g}(x)\right) .
\end{aligned}
$$

Proof This is [34, Theorem 3.28], which adapts [37, Corollary 4.41] to the pointed case. The vertical equivalences come from [37, Proposition 3.48], while the horizontal equivalences are from [37, Theorems 4.39] and Theorem 4.44 or, for a shorter and more conceptual proof, [39, Theorem 6.23]. The results of $[15,4.1]$ imply that $D$ and Th are homotopy inverses.

Definition 2.75 Recall that $O(R)$ has the natural structure of an $R \times R$-representation. Since every $R$-representation has an associated semisimple local system on $|B R(k)|$, we will also write $O(R)$ for the $R$-representation in semisimple local systems on $|B R(k)|$ corresponding to the $R \times R$-representation $O(R)$. We then define the $R-$ representation $\mathbb{O}(R)$ in semisimple local systems on $X$ by $\mathbb{O}(R):=\rho^{-1} O(R)$.

Proposition 2.76 Under the equivalences of Theorem 2.74, the relative Malcev homotopy type $G(X)^{\rho, \text { Mal }}$ of a topological space $X$ corresponds to

$$
\mathrm{C}^{\bullet}(X, \mathbb{O}(R)) \in c \operatorname{Alg}(R),
$$

equipped with its augmentation to $\prod_{x \in X} \mathrm{C}^{\bullet}(x, \mathbb{O}(R)) \cong \prod_{x \in X} O(R)(x,-)$.

Proof This is essentially the same as [37, Theorem 3.55] (which considers the unpointed case).

Corollary 2.77 Pro-algebraic homotopy types are equivalent to the schematic homotopy types of [49], in the sense that the full subcategory of the homotopy category $\mathrm{Ho}(s \mathrm{Pr})$ on objects $X^{\mathrm{sch}}$ is equivalent to the full subcategory of $\mathrm{Ho}(s \mathrm{AGpd})$ on objects $G(X)^{\text {alg }}$. Under this equivalence, $X^{\text {sch }}$ is represented by the simplicial scheme $\bar{W} G(X)^{\mathrm{alg}}$, and pro-algebraic homotopy groups are isomorphic to schematic homotopy groups.

Proof [37, Corollary 3.57]. 
Definition 2.78 Given a manifold $X$, denote the sheaf of real $n$-forms on $X$ by $\mathscr{A}^{n}$. Given a real sheaf $\mathscr{F}$ on $X$, write

$$
A^{n}(X, \mathscr{F}):=\Gamma\left(X, \mathscr{F}_{\mathbb{R}} \otimes_{\mathbb{R}} \mathscr{A}^{n}\right) .
$$

Proposition 2.79 The real Malcev homotopy type of a manifold $X$ relative to $\rho: \pi_{f} X \rightarrow R(\mathbb{R})$ is given in $D G \operatorname{Alg}(R)$ by the de Rham complex $A^{\bullet}(X, \mathbb{O}(R))$, equipped with its augmentation to $\prod_{x \in X} A^{\bullet}(x, \mathbb{O}(R)) \cong \prod_{x \in X} O(R)(x,-)$.

Proof [37, Proposition 4.50].

\section{Pro- $\mathbb{Q}_{\ell}$-algebraic homotopy types}

The purpose if this section is to transfer the framework of Section 2 to an $\ell$-adic setting, replacing topological spaces with pro-finite spaces (and hence étale homotopy types of algebraic varieties).

Fix a prime $\ell$. Although all results here will be stated for the local field $\mathbb{Q}_{\ell}$, they hold for any of its algebraic extensions.

\subsection{Algebraisation of locally pro-finite groupoids}

Definition 3.1 Given a pro-groupoid $\Gamma$ with $\mathrm{Ob}(\Gamma)$ a discrete set (in the sense of Remark 1.10), we define the pro-algebraic completion $\Gamma^{\text {alg }}$ to be the pro- $\mathbb{Q}_{\ell}$-algebraic groupoid pro-representing the functor

$$
\begin{aligned}
\mathrm{AGpd} & \rightarrow \operatorname{Set} \\
H & \mapsto \operatorname{Hom}_{\mathrm{TopGpd}}\left(\Gamma, H\left(\mathbb{Q}_{\ell}\right)\right),
\end{aligned}
$$

where TopGpd denotes the category of topological groupoids, and $H\left(\mathbb{Q}_{\ell}\right)$ is endowed with the topology induced from $\mathbb{Q}_{\ell}$. Note that this exists by the Special Adjoint Functor Theorem [28, Theorem V.8.2], with the algebraic groups $\mathrm{GL}_{n}$ providing the data for the solution set condition (by Tannakian duality). Given a set of primes $L$, define the $L$-algebraic completion $\Gamma^{L \text {,alg }}$ to be $\left(\Gamma^{\wedge} L\right)^{\text {alg }}$. If $P$ is the set of all primes, we simply write $\widehat{\Gamma}^{\text {alg }}:=\Gamma^{P, \text { alg }}$.

Remarks 3.2 Since representations with finite monodromy are algebraic there is a canonical retraction $\Gamma^{L \text {,alg }} \rightarrow \Gamma^{\wedge} L$ of pro-algebraic groupoids.

The motivating example for this definition is when $\Gamma=\pi_{f}^{\text {ét }}(X)$, the étale fundamental groupoid of an algebraic variety. 
The following definition is a slight generalisation of [38, Definition 2.1], and extends Definition 2.20 to pro-groupoids:

Definition 3.3 Given a pro-groupoid $\Gamma$ with $\mathrm{Ob}(\Gamma)$ discrete, a reductive pro-algebraic groupoid $R$ over $\mathbb{Q}_{\ell}$, and a Zariski-dense (ie essentially surjective on objects and Zariski-dense on morphisms) continuous map

$$
\rho: \Gamma^{\wedge} L \rightarrow R\left(\mathbb{Q}_{\ell}\right)
$$

where the latter is given the $\ell$-adic topology, we define the relative Malcev completion $\Gamma^{L, \rho, \mathrm{Mal}}$ (or $\Gamma^{L, R, \mathrm{Mal}}$ ) to be the universal diagram

$$
\Gamma^{\wedge} L \stackrel{g}{\rightarrow} \Gamma^{L, \rho, \mathrm{Mal}}\left(\mathbb{Q}_{\ell}\right) \stackrel{f}{\rightarrow} \widetilde{R}\left(\mathbb{Q}_{\ell}\right),
$$

where $\widetilde{R}$ is the groupoid equivalent to $R$ on objects Ob $\Gamma$ (as in Definition 2.20), with $f: \Gamma^{L, \rho, \mathrm{Mal}} \rightarrow \widetilde{R}$ a pro-unipotent extension of pro- $\mathbb{Q}_{\ell}$-algebraic groupoids, $g$ a continuous map of topological groupoids, and their composition equal to $\rho$.

To see that this universal object exists, we note that this description determines the linear representations of $\Gamma^{L, \rho, \mathrm{Mal}}$ (as described in Remarks 3.4). Since these form a multifibred tensor category, Tannakian duality [37, Remark 2.6] then gives a construction of $\Gamma^{L, \rho, \mathrm{Mal}}$.

Remarks 3.4 By considering groupoid homomorphisms $\Gamma^{\wedge_{L}} \rightarrow \bigsqcup_{n} \mathrm{GL}_{n}\left(\mathbb{Q}_{\ell}\right)$, observe that finite-dimensional linear representations of $\Gamma^{L \text {,alg }}$ are just continuous $\mathbb{Q}_{\ell}-$ representations of $\Gamma^{\wedge L}$.

Finite-dimensional representations of $\Gamma^{L, \rho, \mathrm{Mal}}$ are only those continuous $\mathbb{Q}_{\ell}$-representations whose semisimplifications are $R$-representations. Moreover, if we let $R$ be the reductive quotient $\Gamma^{L \text {,red }}$ of $\Gamma^{L \text {,alg }}$, then $\Gamma^{L, \text { alg }}=\Gamma^{L, R \text {,Mal }}$.

Definition 3.5 Given an $n$-dimensional $\mathbb{Q}_{\ell}$-vector space $V$, a lattice $\Lambda$ in $V$ is a rank $n \mathbb{Z}_{\ell}$-submodule $\Lambda \subset V$.

Lemma 3.6 If $\Gamma$ is a pro-finite group, $V$ an $n$-dimensional $\mathbb{Q}_{\ell}$-vector space, and $\rho: \Gamma \rightarrow \mathrm{GL}(V)$ a continuous representation (where the latter is given the $\ell$-adic topology) then there exists a lattice $\Lambda \subset V$ such that $\rho$ factors through $\operatorname{GL}(\Lambda)$.

Proof Since $\Gamma$ is pro-finite, it is compact, and hence $\rho(\Gamma) \leq \mathrm{GL}(V)$ must be compact. [46, LG 4, Appendix 1, Theorems 1 and 2] show that every compact subgroup of $\mathrm{GL}(V)$ is contained in a maximal compact subgroup, and that the maximal compact subgroups are of the form $\operatorname{GL}(\Lambda)$. Explicitly, we choose a lattice $\Lambda_{0} \subset V$, then set $\Lambda=\sum_{\gamma \in \Gamma} \rho(\gamma) \Lambda_{0}$ (with compactness ensuring the sum is finite). 
Remark 3.7 In particular, when $\Gamma=\pi_{f}^{\text {ét }}(X)$, this means that finite-dimensional representations of $\Gamma^{\text {alg }}$ are smooth $\mathbb{Q}_{\ell}$-sheaves on $X$, while finite-dimensional representations of $\Gamma^{\text {red }}$ are semisimple $\mathbb{Q}_{\ell}$-sheaves. The Zariski-dense map $\rho: \Gamma \rightarrow R\left(\mathbb{Q}_{\ell}\right)$ identifies $R$-representations with a full tensor subcategory of semisimple $\mathbb{Q}_{\ell}$-sheaves, and $\Gamma^{\rho, \mathrm{Mal}}$-representations are Artinian extensions of these semisimple sheaves.

Proposition 3.8 Given a locally pro-finite groupoid $\Gamma$ with discrete objects (as in Remark 1.10), and a Zariski-dense continuous map

$$
\rho: \Gamma^{\wedge}{ }_{L} \rightarrow G\left(\mathbb{Q}_{\ell}\right)
$$

to a pro- $\mathbb{Q}_{\ell}$-algebraic groupoid, there is a canonical model $G_{\mathbb{Z}_{\ell}}$ for $G$ over $\mathbb{Z}_{\ell}$ for which $\rho$ factors through a Zariski-dense map

$$
\rho_{\mathbb{Z}_{\ell}}: \Gamma^{\wedge}{ }_{L} \rightarrow G_{\mathbb{Z}_{\ell}}\left(\mathbb{Z}_{\ell}\right) .
$$

Proof Assume that $\rho$ is an isomorphism on objects (replacing $G$ by an equivalent groupoid). Let $\mathcal{C}$ be the category of continuous $\Gamma$-representations in finite free $\mathbb{Z}_{\ell}-$ modules. For each $x \in \mathrm{Ob} \Gamma$, this gives a fibre functor $\omega_{x}$ from $\mathcal{C}$ to finite free $\mathbb{Z}_{\ell}$-modules.

If we let $\mathcal{D}$ be the category of $\Gamma$-representations in finite-dimensional $\mathbb{Q}_{\ell}$-vector spaces, with the fibre functors also denoted by $\omega_{x}$, then the category of $G$-representations is equivalent to a full subcategory $\mathcal{D}(G)$ of $\mathcal{D}$, since $\rho$ is Zariski-dense. By Tannakian duality (as in [37, Section 2.1]), there are isomorphisms

$$
G(x, y)(A) \cong \operatorname{Iso}^{\otimes}\left(\left.\omega_{x}\right|_{\mathcal{D}(G)},\left.\omega_{y}\right|_{\mathcal{D}(G)}\right)(A),
$$

where Iso ${ }^{\otimes}$ is the set of natural isomorphisms of tensor functors.

Now, by Lemma 3.6, the functor $\otimes \mathbb{Q}_{\ell}: \mathcal{C} \rightarrow \mathcal{D}$ is essentially surjective. Let $\mathcal{C}(G)$ be the full subcategory of $\mathcal{C}$ whose objects are those $\Lambda$ for which $\Lambda \otimes \mathbb{Q}_{\ell}$ is isomorphic to an object of $\mathcal{D}(G)$; these are $\Gamma$-lattices in $G$-representations. Define

$$
G_{\mathbb{Z}_{\ell}}(x, y)(A):=\operatorname{Iso}^{\otimes}\left(\left.\omega_{x}\right|_{\mathcal{C}(G)},\left.\omega_{y}\right|_{\mathcal{C}(G)}\right)(A),
$$

observing that this is an affine scheme (since it preserves all limits), with $G_{\mathbb{Z}_{\ell}} \otimes \mathbb{Q}_{\ell}=G$.

Equivalently, we could set $O\left(G_{\mathbb{Z}_{\ell}}\right) \subset O(G)$ to be $\left\{f L f(\rho(\gamma)) \in \mathbb{Z}_{\ell} \forall \gamma \in \Gamma\right\}$.

Definition 3.9 Given a finite-dimensional nilpotent Lie algebra $\mathfrak{u}$ over $\mathbb{Q}_{\ell}$, equipped with the continuous action of a pro-finite group $\Gamma$ (respecting the Lie algebra structure), we say that a lattice $\Lambda \subset \mathfrak{u}$ is admissible if it satisfies the following: 
(1) $\Lambda$ is a $\Gamma$-subrepresentation.

(2) $\Lambda$ is closed under all the monomials in the Campbell-Baker-Hausdorff formula $\log \left(e^{a} \cdot e^{b}\right)=\sum_{n>0} \frac{(-1)^{n-1}}{n} \sum_{\substack{r_{i}+s_{i}>0 \\ 1 \leq i \leq n}} \frac{\left(\sum_{i=1}^{n}\left(r_{i}+s_{i}\right)\right)^{-1}}{r_{1} ! s_{1} ! \cdots r_{n} ! s_{n} !}\left[a^{r_{1}} b^{s_{1}} a^{r_{2}} b^{s_{2}} \ldots a^{r_{n}} b^{s_{n}}\right]$, where

$$
\begin{aligned}
{\left[a^{r_{1}} b^{s_{1}} \ldots a^{r_{n}} b^{s_{n}}\right]=} & {[\overbrace{a,[a, \ldots[a}^{r_{1}}, \overbrace{b,[b, \ldots[b}^{r_{1}}, \ldots[\overbrace{[a,[a, \ldots[a}^{s_{1}},[\overbrace{b,[b, \ldots b}^{r_{n}}] \ldots]], } \\
\text { understood to be } 0 & \text { if } s_{n}>1 \text { or if } s_{n}=0 \text { and } r_{n}>1 .
\end{aligned}
$$

Lemma 3.10 If $\Lambda \subset \mathfrak{u}$ is an admissible lattice and $\mathfrak{u} \in \mathcal{N}$, then the image of $\Lambda$ under the exponential map

$$
\exp : \mathfrak{u} \rightarrow \exp (\mathfrak{u})
$$

is a pro-finite subgroup.

Proof We may regard $\exp (\mathfrak{u})$ as being the set $\mathfrak{u}$, with multiplication given by the Campbell-Baker-Hausdorff formula (which has only finitely many terms in this case, since $\mathfrak{u}$ is nilpotent). Since $\Lambda$ is closed under all the operations in the formula, it is closed under multiplication. As exp is a homeomorphism, $\exp (\Lambda)$ is compact and thus pro-finite.

\subsection{Pro- $\mathbb{Q}_{\ell}$-algebraic homotopy types}

We now proceed as in Section 2.2, extending to a simplicial framework in order to study the loop groupoid (and hence the whole homotopy type), rather than just the fundamental groupoid.

Definition 3.11 Given a pro-simplicial groupoid $G$ with $\mathrm{Ob}(G)$ a discrete set, we define the pro-algebraic completion $G^{L \text {,alg }} \in S$ AGpd to represent the functor

$$
\begin{aligned}
s \mathrm{AGpd} & \rightarrow \text { Set } \\
H & \mapsto \operatorname{Hom}_{s \operatorname{TopGpd}}\left(G^{\wedge} L, H\left(\mathbb{Q}_{\ell}\right)\right),
\end{aligned}
$$

where TopGpd denotes the category of topological groupoids. Note that Lemma 1.17 implies that we can compute this levelwise by $\left(G^{L, \text { alg }}\right)_{n}=\left(G_{n}\right)^{L \text {,alg }}$.

Remark 3.12 It is natural to ask whether $G \mapsto G^{L \text {,alg }}$ is left Quillen for any suitable model structure on pro- $L$ simplicial groupoids. This cannot be the case, since the functor is not even a left adjoint, essentially because $\mathbb{Q}_{\ell}$ is not pro-finite. 
Definition 3.13 Given a pro-simplicial groupoid $G$ with $\mathrm{Ob}(G)$ discrete, a reductive pro-algebraic groupoid $R$ over $\mathbb{Q}_{\ell}$, and a Zariski-dense continuous map

$$
\rho: \pi_{0}(G)^{\wedge} \rightarrow R\left(\mathbb{Q}_{\ell}\right),
$$

where the latter is given the $\ell$-adic topology, we define the relative Malcev completion $G^{L, \rho, \mathrm{Mal}} \in S \mathcal{E}(R) \subset s \mathrm{AGpd} \downarrow R$ by $\left(G^{L, \rho, \mathrm{Mal}}\right)_{n}:=\left(G_{n}\right)^{L, \rho \circ a_{n}, \mathrm{Mal}}$, for $a_{n}: G_{n} \rightarrow \pi_{0} G$ the canonical map.

Note that $\pi_{0}\left(G^{L, \rho, \mathrm{Mal}}\right)=\pi_{0}(G)^{L, \rho, \mathrm{Mal}}$.

Lemma 3.14 If the continuous action of a pro-finite group $\Gamma$ on $\mathfrak{u}_{\bullet} \in s \mathcal{N}_{\mathbb{Q}_{\ell}}$ is semisimple, then $\mathfrak{u}$ is the union of its $\Gamma$-equivariant simplicial admissible sublattices.

Proof Since the action of $\Gamma$ is semisimple, we may take a complement $V \bullet \subset \mathfrak{u}_{\bullet}$ of $\left[\mathfrak{u}_{\bullet}, \mathfrak{u}_{\bullet}\right]$ as a simplicial $\Gamma$-representation. Given a lattice $M \subset V$, let $g(M) \subset \mathfrak{u}$ denote the $\mathbb{Z}_{\ell}$-submodule generated by $M$ and the operations in the Campbell-BakerHausdorff formula. Since $\mathfrak{u}$ is nilpotent, it follows that $g(M)$ is a finitely generated $\mathbb{Z}_{\ell}$-module, and hence a lattice in $\mathfrak{u}$. By semisimplicity and Lemma 3.6, there exists a $\Gamma$-equivariant lattice $\Lambda_{\bullet} \subset V_{\bullet}$. The lattices $\ell^{-n} \Lambda_{\bullet} \subset V_{\bullet}$ are also then $\Gamma$-equivariant for $n \geq 0$, so the lattices $g\left(\ell^{-n} \Lambda_{\bullet}\right) \subset \mathfrak{u}_{\bullet}$ are all admissible.

It only remains to show that $\bigcup g\left(\ell^{-n} \Lambda\right) \rightarrow \mathfrak{u}$ is a surjective map of Lie algebras. This follows since $\bigcup \ell^{-n} \Lambda \rightarrow \mathfrak{u} /[\mathfrak{u}, \mathfrak{u}]$ is surjective.

Lemma 3.15 Given a compact topological space $K$ and a finite-dimensional nilpotent $\mathbb{Q}_{\ell}$-Lie algebra $\mathfrak{u}$, the map

$$
\operatorname{Hom}_{\text {cts }}\left(K, \mathbb{Z}_{\ell}\right) \otimes_{\mathbb{Z}_{\ell}} \mathfrak{u} \rightarrow \operatorname{Hom}_{\text {cts }}(K, \mathfrak{u})
$$

is an isomorphism.

Proof First observe that the map is clearly injective, since $\mathfrak{u}$ is a flat $\mathbb{Z}_{\ell}$-module. For surjectivity, note that the image of $f: K \rightarrow \mathfrak{u}$ must be contained in an admissible sublattice $\Lambda \subset \mathfrak{u}$ (by compactness and Lemma 3.14). Now,

$$
\operatorname{Hom}_{\mathrm{cts}}(K, \Lambda) \cong \operatorname{Hom}_{\mathrm{cts}}\left(K, \mathbb{Z}_{\ell}\right) \otimes_{\mathbb{Z}_{\ell}} \Lambda,
$$

since $\Lambda$ is a finite free $\mathbb{Z}_{\ell}$-module.

Definition 3.16 Given a continuous representation $V$ of $\widehat{\pi_{f} X}$ in $\mathbb{Q}_{\ell}$-vector spaces, recall the standard definition that

$$
\mathrm{H}^{*}(X, V):=\mathrm{H}^{*}(X, \Lambda) \otimes_{\mathbb{Z}_{\ell}} \mathbb{Q}_{\ell},
$$


for any $\pi_{f} X$-equivariant $\mathbb{Z}_{\ell}$-lattice $\Lambda \subset V$ as in Lemma 3.6, and $\mathrm{H}^{*}(X, \Lambda)$ as in Definition 1.23.

Remark 3.17 If $X$ is discrete, note that this is not in general the same as cohomology $\mathrm{H}^{n}\left(X, V^{\delta}\right)$ of the discrete $\pi_{f} X$-representation $V^{\delta}$ underlying $V$. However, both will coincide if $\mathrm{H}_{n}\left(G, \Lambda^{\vee}\right)$ has finite rank, by the Universal Coefficient Theorem and Lemma 1.25 .

Example 3.18 If $X$ is a locally Noetherian simplicial scheme, we may consider the étale topological type $X_{\text {ét }} \in \operatorname{pro}(\mathbb{S})$, as defined in [10, Definition 4.4]. Since $\left(X_{\text {et }}\right)_{0}$ is the set of geometric points of $X_{0}$, we may then apply the constructions of this section. For a finite local system $M$ on $X$, we have

$$
\mathrm{H}^{*}\left(X_{\text {ét }}, M\right) \cong \mathrm{H}_{\text {et }}^{*}(X, M),
$$

by [10, Proposition 5.9]. For an inverse system $M=\left\{M_{i}\right\}$ of local systems, we have

$$
\mathrm{H}^{*}\left(X_{\text {ét }}, M\right)=\mathrm{H}^{*}\left(\underset{i}{\lim } \mathrm{C}_{\text {ét }}^{\bullet}\left(X, M_{i}\right)\right)=\mathrm{H}_{\mathrm{et}}^{*}(X,(M)),
$$

where $\mathrm{C}_{\mathrm{e} t}^{\bullet}$ is a variant of the Godement resolution and $\mathrm{H}_{\mathrm{et}}^{*}(X,(M))$ is Jannsen's continuous étale cohomology [20]. If the groups $\mathrm{H}_{\mathrm{et}}^{*}\left(X, M_{i}\right)$ satisfy the Mittag-Leffler condition (in particular, if they are finite), then

$$
\mathrm{H}^{*}\left(X_{\text {ét }}, M\right) \cong \underset{i}{\lim } \mathrm{H}_{\text {et }}^{*}\left(X, M_{i}\right)
$$

[10, Theorem 7.3] shows that $X_{\text {ét }} \in \widehat{\mathbb{S}}$ whenever the schemes $X_{n}$ are connected and geometrically unibranched. It seems that this result can be extended to simplicial schemes (or even simplicial algebraic spaces) for which the homotopy groups $\pi_{m}^{\text {ét }}\left(X_{n}\right)$ satisfy the $\pi_{*}$-Kan condition [11, Section IV.4], provided the simplicial set $\pi(X) \bullet$, given by $\pi(X)_{n}:=\pi\left(X_{n}\right)$, the set of connected components of $X_{n}$, has finite homotopy groups.

Proposition 3.19 Take $X \in \operatorname{pro}(\mathbb{S})$ with $X_{0}$ discrete, and a Zariski-dense continuous map

$$
\rho: \pi_{f}(X)^{\wedge L} \rightarrow R\left(\mathbb{Q}_{\ell}\right),
$$

for $\ell \in L$, with $\mathrm{Ob} R=\mathrm{Ob} \pi_{f}(X)$. Then $G(X)^{L, \rho, \mathrm{Mal}}$ is cofibrant (for the model structure of Lemma 2.16), the map $G(X)^{L^{\prime}, \rho, \mathrm{Mal}} \rightarrow G(X)^{L, \rho, \mathrm{Mal}}$ is an isomorphism for all $L \subset L^{\prime}$, and

$$
\mathrm{H}^{*}\left(G(X)^{L, \rho, \mathrm{Mal}}, V\right) \cong \mathrm{H}^{*}\left(X, \rho^{*} V\right)
$$


Proof Let $\Delta \leq R\left(\mathbb{Q}_{\ell}\right)$ be the image of $\rho$. Write $\left\{X_{\alpha}\right\}_{\alpha \in \mathbb{I}}$ for the inverse system $X$. For $\mathfrak{u} \in s \mathcal{N}(R)$,

$$
\operatorname{Hom}_{s \text { TopGpd }}\left(G(X)^{\wedge L}, \exp (\mathfrak{u}) \rtimes R\right)_{R}=\operatorname{Hom}_{s \operatorname{TopGpd}}\left(G(X)^{\wedge L}, \exp (\mathfrak{u}) \rtimes \Delta\right)_{\Delta} .
$$

Since $\mathfrak{u} \in s \mathcal{N}(R)$, the normalisation $N \mathfrak{u}$ is bounded in degrees $\leq n$, say. This implies that $\mathfrak{u}=\operatorname{cosk}_{n+1} \mathfrak{u}$, the $(n+1)$-coskeleton, or equivalently that any simplicial morphism $Y \rightarrow \mathfrak{u}$ is determined by the maps $Y_{i} \rightarrow \mathfrak{u}_{i}$ for $i \leq n+1$.

So any morphism $f: G(X)^{\wedge} L \rightarrow \exp (\mathfrak{u}) \rtimes \Delta$ is determined by the maps $f_{i}: G(X)_{i}^{\wedge} \rightarrow$ $\exp \left(\mathfrak{u}_{i}\right) \rtimes \Delta$ for $i \leq n+1$. Now, by Lemma 3.14, $\exp (\mathfrak{u}) \rtimes \Delta$ is the union over all admissible $\Delta$-equivariant sublattices $\Lambda \subset \mathfrak{u}$ of $\exp (\Lambda) \rtimes \Delta$. Since each $G(X)_{i}^{\wedge^{L}}$ is compact, its image in $\exp \left(\mathfrak{u}_{i}\right) \rtimes \Delta$ must be contained in $\exp \left(\Lambda_{i}\right) \rtimes \Delta$ for some admissible $\Lambda \subset \mathfrak{u}$. By choosing $\Lambda$ large enough that this holds for all $i \leq n+1$, we see that

$$
\begin{aligned}
\operatorname{Hom}_{s \text { TopGpd }}\left(G(X)^{\wedge} L,\right. & \exp (\mathfrak{u}) \rtimes R)_{R} \\
& =\underset{\Lambda \subset \mathfrak{u} \text { admissible }}{\underset{\lim }{\longrightarrow}} \operatorname{Hom}_{s \operatorname{TopGpd}}\left(G(X)^{\wedge}, \exp (\Lambda) \rtimes \Delta\right)_{\Delta} \\
& =\underset{\Lambda \subset \mathfrak{u} \text { admissible }}{\underset{\lim }{\longrightarrow}} \operatorname{Hom}_{s \operatorname{pro}\left(\mathrm{Gpd}^{L}\right)}\left(G(X)^{\wedge L}, \exp (\Lambda) \rtimes \Delta\right)_{\Delta},
\end{aligned}
$$

because $\operatorname{pro}\left(\mathrm{Gpd}^{L}\right)$ is a full subcategory of TopGpd. Here, $\exp (\Lambda) \rtimes \Delta \in \operatorname{pro}\left(s \mathrm{Gpd}^{L}\right)$ denotes the pro-object $\left\{\left(\exp (\Lambda) / \exp \left(\ell^{m} \Lambda\right)\right) \rtimes \Delta\right\} m$. From now on, we will abuse notation by writing $\exp \left(\Lambda / \ell^{n} \Lambda\right)$ or $\operatorname{even} \exp \left(\Lambda / \ell^{n}\right)$ for the finite group $\exp (\Lambda) / \exp \left(\ell^{n} \Lambda\right)$.

Now, since $\Lambda=\operatorname{cosk}_{n+1} \Lambda$, any morphism $f: H \rightarrow \exp \left(\Lambda / \ell^{m} \Lambda\right) \rtimes \Delta$ is determined by the maps $f_{i}$ for $i \leq n+1$. As $\exp \left(\Lambda / \ell^{m} \Lambda\right)$ is levelwise finite, and filtered colimits commute with finite limits, this means that

$\operatorname{Hom}_{s \operatorname{pro}\left(\operatorname{Gpd}^{L}\right)}\left(G(X)^{\wedge L}, \exp (\Lambda) \rtimes \Delta\right)_{\Delta}=\operatorname{Hom}_{\operatorname{pro}\left(s \operatorname{Gpd}^{L}\right)}\left(G(X)^{\wedge L}, \exp (\Lambda) \rtimes \Delta\right)_{\Delta}$. Hence $\operatorname{Hom}_{s \text { TopGpd }}\left(G(X)^{\wedge L}, \exp (\mathfrak{u}) \rtimes R\right)_{R}$

$$
=\underset{\Lambda \subset \mathfrak{u} \text { admissible }}{\stackrel{\lim }{\longrightarrow}} \operatorname{Hom}_{\operatorname{pro}\left(s \mathrm{Gpd}^{L}\right)}(G(X), \exp (\Lambda) \rtimes \Delta)_{\Delta} .
$$

Under the adjunction $G \dashv \bar{W}$, this becomes

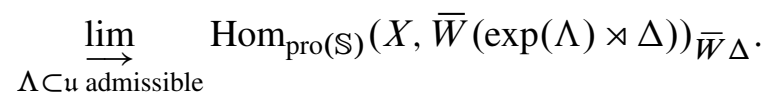

This expression is independent of $L$, so we have shown $G(X)^{L^{\prime}, \rho, \mathrm{Mal}} \rightarrow G(X)^{L, \rho, \mathrm{Mal}}$ is an isomorphism for all $L \subset L^{\prime}$. 
For $p: \mathfrak{u} \rightarrow \mathfrak{v}$ an acyclic small extension with kernel $I$ in $s \mathcal{N}(R)$, and an admissible lattice $\Lambda^{\prime}<\mathfrak{u}$, consider the map $\Lambda^{\prime} \rightarrow p\left(\Lambda^{\prime}\right)$. This is surjective, and $H_{*}\left(\Lambda^{\prime} \cap I\right) \otimes \mathbb{Q}_{\ell}=$ 0 , since $\left(\Lambda^{\prime} \cap I\right) \otimes \mathbb{Q}_{\ell} \cong I$. As $\mathrm{H}_{*}(I)=0$, we may choose a $\Delta$-equivariant lattice $\Lambda^{\prime} \cap I<M<I$ such that $\mathrm{H}_{*}(M / \ell M)=0$. Let $\Lambda:=\Lambda^{\prime}+M$, noting that this is an admissible lattice ( $p$ being small), with the maps $\Lambda / \ell^{n} \rightarrow p(\Lambda) / \ell^{n}$ all acyclic.

In order to show that $G(X)^{L, \rho, \mathrm{Mal}}$ is cofibrant, take an arbitrary map $f: G(X)^{\wedge} L \rightarrow$ $\exp (\mathfrak{v}) \rtimes \Delta$ over $\rho$; this must factor through $\exp \left(p\left(\Lambda^{\prime}\right)\right)$ for some admissible lattice $\Lambda^{\prime}<\mathfrak{u}$, and we may replace $\Lambda$ by $\Lambda^{\prime}$ as above. It therefore suffices to show that the corresponding map

$$
f: X \rightarrow \bar{W}(\exp (p(\Lambda)) \rtimes \Delta)
$$

in $\operatorname{pro}(\mathbb{S})$ lifts to $\bar{W}(\exp (\Lambda) \rtimes \Delta)$. For each $n \in \mathbb{N}$, we have a map

$$
f_{n}: X_{\alpha(n)} \rightarrow \bar{W}\left(\exp \left(p(\Lambda) / \ell^{n}\right) \rtimes \Delta\right),
$$

and these are compatible with the structural morphisms.

We now prove existence of the lift by induction on $n$. Assume we have $g_{n}: X_{\alpha(n)} \rightarrow$ $\bar{W}\left(\exp \left(\Lambda / l^{n}\right) \rtimes \Delta\right)$, such that $p \circ g_{n}=f_{n}$. This gives a map

$$
\left(f_{n+1}, g_{n}\right): X_{\alpha(n)} \rightarrow \bar{W}\left(\exp \left(\left(p(\Lambda) / \ell^{n+1}\right) \times_{p(\Lambda) / \ell^{n}}\left(\Lambda / \ell^{n}\right)\right) \rtimes \Delta\right) .
$$

However, $\Lambda / \ell^{n+1} \rightarrow\left(p(\Lambda) / \ell^{n+1}\right) \times_{p(\Lambda) / \ell^{n}}\left(\Lambda / \ell^{n}\right)$ is an acyclic small extension, so

$$
\bar{W}\left(\exp \left(\Lambda / \ell^{n+1}\right) \rtimes \Delta\right) \rightarrow \bar{W}\left(\exp \left(\left(p(\Lambda) / \ell^{n+1}\right) \times_{p(\Lambda) / \ell^{n}}\left(\Lambda / \ell^{n}\right)\right) \rtimes \Delta\right)
$$

is a trivial fibration, so we can construct a lift $g_{n+1}: X_{\alpha(n+1)} \rightarrow \bar{W}\left(\exp \left(\Lambda / \ell^{n+1}\right) \rtimes \Delta\right)$. This completes the proof that $G(X)^{L, \rho, \text { Mal }}$ is cofibrant.

Finally, if $V$ is an $R$-representation then $\mathrm{H}^{n+1}\left(G(X)^{L, \rho, \mathrm{Mal}}, V\right)$ is the coequaliser of the diagram

$$
\operatorname{Hom}_{S \mathrm{AGpd} \downarrow}\left(G(X)^{L, \rho, \mathrm{Mal}},\left(N^{-1} V[-n]\right)^{\Delta^{1}}\right)
$$

$$
\Longrightarrow \operatorname{Hom}_{s \mathrm{AGpd} \downarrow R}\left(G(X)^{L, \rho, \mathrm{Mal}}, N^{-1} V[-n]\right) .
$$

For a $\Delta$-equivariant lattice $\Lambda \subset V$, this is the direct limit over $m$ of

$\operatorname{Hom}_{\operatorname{pro}(\mathbb{S} \downarrow \bar{W} R)}\left(X, \bar{W}\left(\left(N^{-1} \ell^{-m} \Lambda[-n]\right)^{\Delta^{1}} \rtimes R\right)\right)$

$$
\Longrightarrow \operatorname{Hom}_{\operatorname{pro}(\mathbb{S} \downarrow \bar{W} R)}\left(X, \bar{W}\left(N^{-1} \ell^{-m} \Lambda[-n] \rtimes R\right)\right) .
$$

Hence

$$
\mathrm{H}^{n+1}\left(G(X)^{L, \rho, \mathrm{Mal}}, V\right) \cong \underset{m}{\lim } \mathrm{H}^{n+1}\left(X, l^{-m} \Lambda\right)=\mathrm{H}^{n+1}(X, \Lambda) \otimes \mathbb{Q}_{\ell}=\mathrm{H}^{n+1}(X, V),
$$

as required. 
Definition 3.20 Given $X$ and $\rho$ as above, define the relative Malcev homotopy type

$$
X^{\rho, \mathrm{Mal}}:=G(X)^{P, \rho, \mathrm{Mal}},
$$

where $P$ is the set of all primes, noting that this is isomorphic to $G(X)^{L, \rho, \text { Mal }}$ for all $L \ni \ell$, by Proposition 3.19 and Proposition 2.39.

Define

$$
X^{L, \text { alg }}:=G(X)^{L, \text { alg }} .
$$

Remark 3.21 Note that if $X \in \mathbb{S}$, this definition of Malcev completion differs slightly from the Malcev homotopy type $X^{\rho, \text { Mal }}$ of Definition 2.51, which is given by $G(X)^{\rho, \mathrm{Mal}}$. However, the following lemma rectifies the situation.

Lemma 3.22 For $X \in \mathbb{S}$ and $\rho: \pi_{f}(X)^{\wedge} L \rightarrow R\left(\mathbb{Q}_{\ell}\right)$ Zariski-dense and continuous, there is a canonical map

$$
G(X)^{\rho, \mathrm{Mal}} \rightarrow G(X)^{L, \rho, \mathrm{Mal}}
$$

this is a quasi-isomorphism whenever the groups $\mathrm{H}^{n}(X, V)$ are finite-dimensional for all finite-dimensional $R$-representations $V$.

Proof Existence of the map is immediate. To see that it gives a quasi-isomorphism, Proposition 2.39 shows that we need only look at cohomology groups. Given an $R$-representation $V$ corresponding to a local system $\mathbb{V}$ over $\mathbb{Q}_{\ell}$ on $X$, the map on cohomology groups is

$$
\mathrm{H}^{*}\left(X^{\wedge}, \mathbb{V}\right) \rightarrow \mathrm{H}^{*}(X, \mathbb{V})
$$

this is an isomorphism by Remark 3.17.

Definition 3.23 Define pro-algebraic (or schematic) and relative homotopy groups by $\varpi_{n}\left(X^{\wedge}\right):=\pi_{n-1}\left(G(X)^{L, \text { alg }}\right)$ and $\varpi_{n}\left(X^{\rho, \mathrm{Mal}}\right):=\pi_{n-1}\left(G(X)^{P, \rho, \mathrm{Mal}}\right)$.

Define pro-algebraic (or schematic) and relative fundamental groupoids by

$$
\varpi_{f}\left(X^{\wedge L}\right):=\pi_{f}(X)^{L, \text { alg }} \text { and } \varpi_{f}\left(X^{\rho, \mathrm{Mal}}\right):={\widehat{\pi_{f}}}^{\rho, \mathrm{Mal}} .
$$

Define $\varpi_{f}(\hat{X}), \varpi_{n}(\hat{X})$ by the convention that $\hat{X}=X^{\wedge} P$, for $P$ the set of all primes.

Note that Lemma 3.6 implies that for a locally Noetherian scheme $X$, finite-dimensional $\varpi_{f}\left(X_{\text {ét }}^{\wedge}\right)$-representations correspond to smooth $\mathbb{Q}_{\ell}$-sheaves on $X$.

The following now follow immediately from Proposition 2.39 
Corollary 3.24 A map $f: X \rightarrow Y$ in pro(S), with $X_{0}, Y_{0}$ discrete, induces an isomorphism

$$
f^{L, \text { alg }:} X^{L, \text { alg }} \rightarrow Y^{L, \text { alg }}
$$

of homotopy types if and only if the following conditions hold:

(1) $f^{*}$ induces an equivalence between the categories of finite-dimensional semisimple continuous $\mathbb{Q}_{\ell}$-representations of $\left(\pi_{f} X\right)^{\wedge} L$ and $\left(\pi_{f} Y\right)^{\wedge} L$.

(2) For all finite-dimensional semisimple continuous $\mathbb{Q}_{\ell}$-representations $V$ of $\pi_{f} Y$, the maps

$$
f^{*}: \mathrm{H}^{*}(Y, V) \rightarrow \mathrm{H}^{*}\left(X, f^{*} V\right)
$$

are isomorphisms.

Corollary 3.25 Take a map $f: X \rightarrow Y$ in pro(S), with $X_{0}, Y_{0}$ discrete, and with a Zariski-dense morphism $\rho:\left(\pi_{f} Y\right)^{\wedge} L \rightarrow R\left(\mathbb{Q}_{\ell}\right)$ such that $\rho \circ f:\left(\pi_{f} X\right)^{\wedge} L \rightarrow R\left(\mathbb{Q}_{\ell}\right)$ is also Zariski-dense. Then $f$ induces an isomorphism

$$
f^{R, \mathrm{Mal}}: X^{R, \mathrm{Mal}} \rightarrow Y^{R, \mathrm{Mal}}
$$

of homotopy types if and only if for all $R$-representations $V$, the maps

$$
f^{*}: \mathrm{H}^{*}\left(Y, \rho^{*} V\right) \rightarrow \mathrm{H}^{*}\left(X, f^{*} \rho^{*} V\right)
$$

are isomorphisms.

\subsection{Equivariant cochains}

Proposition 2.79 showed how the schematic homotopy type of a manifold can be recovered from the de Rham complex with local system coefficients. We will now establish an analogue for algebraic varieties, involving an étale Godement resolution with coefficients in smooth $\mathbb{Q}_{\ell}$-sheaves.

Lemma 3.26 If $\Lambda$ is a $\Gamma$-representation in pro-simplicial groups such that $\Lambda \rtimes \Gamma \in$ $\operatorname{pro}(s \mathrm{Gpd})$, then

$$
\operatorname{Hom}_{\Gamma, \operatorname{pro}(\mathbb{S})}(\tilde{X}, \bar{W} \Lambda) \cong \operatorname{Hom}_{\operatorname{pro}(\mathbb{S}) \downarrow B \Gamma}(X, \bar{W}(\Lambda \rtimes \Gamma)),
$$

for $\tilde{X}$ as in Definition 1.20 .

Proof The calculation is essentially the same as for [37, Lemma 3.53]. 
Definition 3.27 Given an ind-finite rank $\mathbb{Z}_{\ell}$-local system (ie a filtered direct system in the category of finite rank $\mathbb{Z}_{\ell}$-local systems) $\mathbb{V}=\left\{\mathbb{V}_{\alpha}\right\}_{\alpha}$, define

$$
\mathrm{C}^{\bullet}(X, \mathbb{V}):=\underset{\alpha}{\lim } \mathrm{C}^{\bullet}\left(X, \mathbb{V}_{\alpha}\right),
$$

where the right-hand side is given in Definition 1.21.

Definition 3.28 Given a pro-algebraic groupoid $G$ over $\mathbb{Z}_{\ell}$, define $O(G)$ to be the $G \times G$-representation given by global sections of the structure sheaf of $G$, equipped with its left and right $G$-actions.

Given a representation $\rho: \pi_{f} X \rightarrow G\left(\mathbb{Z}_{\ell}\right)$, let $\mathbb{O}(G)$ be the $G$-representation in (ind-finite rank) $\mathbb{Z}_{\ell}$-local systems on $X$ given by pulling $O(G)$ back along its right $G$-action.

Definition 3.29 Given $X, L, \rho, R$ as in Proposition 3.19, let $R_{\mathbb{Z}_{\ell}}$ be the $\mathbb{Z}_{\ell}$-model for $R$ constructed in Proposition 3.8, and set

$$
\mathrm{C}^{\bullet}(X, \mathbb{O}(R)):=\mathrm{C}^{\bullet}\left(X, \mathbb{O}\left(R_{\mathbb{Z}_{\ell}}\right)\right) \otimes_{\mathbb{Z}_{\ell}} \mathbb{Q}_{\ell} .
$$

Theorem 3.30 For $X, L, \rho, R$ as in Proposition 3.19, the relative Malcev homotopy type

$$
G(X)^{L, \rho, \text { Mal }} \in s \mathrm{AGpd} \downarrow R
$$

corresponds under the equivalences of Proposition 2.50 and Theorem 2.74 to the $R-$ representation

$$
\mathrm{C}^{\bullet}(X, \mathbb{O}(R))
$$

in cosimplicial $k$-algebras, equipped with its natural augmentation to

$$
\prod_{x \in X_{0}} \mathrm{C}^{\bullet}(x, \mathbb{O}(R))=\prod_{x \in \mathrm{Ob} R} O(R)(x,-) .
$$

Proof We need to show that, for $\mathfrak{u} \in s \mathcal{N}(R)$,

$\operatorname{Hom}_{s \mathrm{AGpd} \downarrow}\left(G(X)^{L, \rho, \mathrm{Mal}}, \exp (\mathfrak{u}) \rtimes R\right)$

$$
\cong \operatorname{Hom}_{s} \operatorname{Aff}(R)\left(\operatorname{Spec}^{\bullet}(X, \mathbb{O}(R)), \bar{W}(\exp (\mathfrak{u}))\right) .
$$

Adapting the proof of Proposition 3.19, we know that

$\operatorname{Hom}_{S \mathrm{AGpd} \downarrow R}\left(G(X)^{L, \rho, \mathrm{Mal}}, \exp (\mathfrak{u}) \rtimes R\right)$

$$
\cong \underset{\Lambda}{\lim _{\longrightarrow}} \operatorname{Hom}_{\operatorname{pro}(\mathbb{S})}\left(X, \bar{W}\left(\exp (\Lambda) \rtimes R_{\mathbb{Z}_{\ell}}\left(\mathbb{Z}_{\ell}\right)\right)\right)_{B R_{\mathbb{Z}_{\ell}}\left(\mathbb{Z}_{\ell}\right)},
$$


where the limit is taken over $\Lambda \subset \mathfrak{u}$ admissible. By Lemma 3.26,

$\operatorname{Hom}_{\operatorname{pro}(\mathbb{S})}\left(X, \bar{W}\left(\exp (\Lambda) \rtimes R_{\mathbb{Z}_{\ell}}\left(\mathbb{Z}_{\ell}\right)\right)\right)_{B R_{\mathbb{Z}_{\ell}}\left(\mathbb{Z}_{\ell}\right)} \cong \operatorname{Hom}_{R_{\mathbb{Z}_{\ell}}\left(\mathbb{Z}_{\ell}\right), \operatorname{pro}(\mathbb{S})}(\tilde{X}, \bar{W} \exp (\Lambda))$.

If we regard $\exp (\Lambda)$ as the $\mathbb{Z}_{\ell}$-valued points of the group scheme $\exp (\Lambda)(A):=$ $\exp (\Lambda \otimes A)$, then this is an affine space, so

$$
\operatorname{Hom}_{\operatorname{pro}(\mathbb{S})}(\tilde{X}, \bar{W} \exp (\Lambda)) \cong \operatorname{Hom}_{s} \operatorname{Aff}_{\mathbb{Z}_{\ell}}\left(\operatorname{Spec} C^{\bullet}\left(\tilde{X}, \mathbb{Z}_{\ell}\right), \bar{W} \exp (\Lambda)\right) .
$$

Since $\Lambda \cong \Lambda \otimes_{\mathbb{Z}_{\ell}}^{R} O\left(R_{\mathbb{Z}_{\ell}}\right)$, we then have

$$
\begin{aligned}
& \operatorname{Hom}_{R_{\mathbb{Z}_{\ell}}\left(\mathbb{Z}_{\ell}\right), \operatorname{pro}(\mathbb{S})}(\tilde{X}, \bar{W} \exp (\Lambda)) \\
& \qquad \operatorname{Hom}_{s \operatorname{Aff}\left(R_{\mathbb{Z}_{\ell}}\right)}\left(\operatorname{Spec} C^{\bullet}\left(X, \mathbb{O}\left(R_{\mathbb{Z}_{\ell}}\right)\right), \bar{W} \exp (\Lambda)\right) .
\end{aligned}
$$

The map

$$
\begin{aligned}
\underset{\Lambda}{\lim } \operatorname{Hom}_{s \operatorname{Aff}\left(R_{\mathbb{Z}_{\ell}}\right)}\left(\operatorname{Spec}^{\bullet}\left(X, \mathbb{O}\left(R_{\mathbb{Z}_{\ell}}\right)\right), \bar{W} \exp (\Lambda)\right) \\
\quad \rightarrow \underset{\Lambda}{\lim } \operatorname{Hom}_{s \operatorname{Aff}\left(R_{\mathbb{Z}_{\ell}}\right)}\left(\operatorname{Spec} C^{\bullet}\left(X, \mathbb{O}\left(R_{\mathbb{Z}_{\ell}}\right)\right) \otimes \mathbb{Q}_{\ell}, \bar{W} \exp (\Lambda)\right)
\end{aligned}
$$

is clearly injective. However, since there exists an admissible lattice $\Lambda^{\prime}$ with $l^{-n} \Lambda \subset \Lambda^{\prime}$, the map must also be surjective. Finally, note that

$$
\begin{aligned}
& \operatorname{Hom}_{s \operatorname{Aff}\left(R_{\mathbb{Z}_{\ell}}\right)}\left(\operatorname{Spec} C^{\bullet}\left(X, \mathbb{O}\left(R_{\mathbb{Z}_{\ell}}\right)\right) \otimes \mathbb{Q}_{\ell}, \bar{W} \exp (\Lambda)\right) \\
& =\operatorname{Hom}_{s} \operatorname{Aff}(R)\left(\operatorname{Spec} C^{\bullet}(X, \mathbb{O}(R)), \bar{W} \exp \left(\Lambda \otimes \mathbb{Q}_{\ell}\right)\right),
\end{aligned}
$$

as required.

Remarks 3.31 We could use Proposition 2.70 to replace $C^{\bullet}(X, \mathbb{O}(R))$ with a DG algebra, giving a more reassuring analogue of the de Rham algebra used in Proposition 2.79 to govern relative Malcev homotopy types of manifolds. This is the approach taken by Olsson [33], and when $R=1$, it corresponds to Deligne's $\mathbb{Q}_{\ell}$-homotopy type [5, Section V]. However, in the sequel we will work systematically with cosimplicial rather than DG objects - both approaches being equivalent, the transfer can add unnecessary complication.

Note that if we take a scheme $X$, then Proposition 2.76 adapts to show that $\mathrm{C}^{\bullet}\left(X_{\text {ét }}, \mathbb{V}\right)$ is a Godement resolution for the continuous étale cohomology of $\mathbb{V}$. Under the comparison of Corollary 2.77 , this shows that for an algebraic variety $X, \widehat{G\left(X_{\text {et }}\right)^{\text {alg }}}$ agrees with the $\ell$-adic homotopy type discussed in [49, Section 3.5.3]. 
Given any morphism $\rho: \varpi_{f}\left(\widehat{X_{\text {et }}}\right)^{\text {red }} \rightarrow R$ to a reductive group, there is a forgetful functor $\rho^{\sharp}: s \hat{\mathcal{N}}(R) \rightarrow s \hat{\mathcal{N}}\left(\varpi_{f}\left(\widehat{X_{\text {et }}}\right)^{\text {red }}\right)$. If we write $\mathbb{L} \rho_{\sharp}$ for the derived left adjoint and $\rho$ is surjective, then

$$
\left.\mathrm{R}_{\mathrm{u}}\left(\widehat{G\left(X_{\text {ét }}\right)}\right)^{\rho, \mathrm{Mal}}\right)=\mathbb{L} \rho_{\sharp} \mathrm{R}_{\mathrm{u}}\left(\widehat{G\left(X_{\text {ét }}\right)^{\mathrm{alg}}}\right) .
$$

Note that for $\mathcal{C}$ a Tannakian subcategory (see Definition 2.6) of FD $\operatorname{Rep}\left(\varpi_{f}\left({\widehat{X_{\mathrm{et}}}}^{\mathrm{red}}\right)\right.$, with corresponding groupoid $G$, the homotopy type $X_{\mathcal{C}_{\text {ét }}}$ of $[33,1.5]$ is equivalent to $\mathbb{L} \rho_{\sharp} \mathrm{R}_{\mathrm{u}}\left(\widehat{G(X)^{\mathrm{alg}}}\right)$, for $\rho: \varpi_{f}\left({\widehat{X_{\mathrm{et}}}}^{\mathrm{red}} \rightarrow G\right.$.

\subsection{Completing fibrations}

Observe that the definitions and results of Section 2.4 extend naturally to pro-groupoids and pro-spaces; we will make use of this extension without further comment.

Theorem 3.32 Take a pro-fibration $f:(X, x) \rightarrow(Y, y)$ of connected objects in $\operatorname{pro}(\mathbb{S})$ with connected fibres, and set $F:=f^{-1}(y)$. Take a Zariski-dense representation $\rho: \pi_{1}(X, x) \rightarrow R\left(\mathbb{Q}_{\ell}\right)$ to a reductive pro-algebraic group $R$, let $K$ be the Zariski closure of $\rho\left(\pi_{1}(F, x)\right)$, and set $T:=R / K$. If the monodromy action of $\pi_{1}(Y, y)$ on $\mathrm{H}^{*}(F, V)$ factors through $\varpi_{1}(Y, y)^{T, \text { Mal }}$ for all $K$-representations $V$, then $G(F, x)^{K \text {,Mal }}$ is the homotopy fibre of $G(X, x)^{R, \text { Mal }} \rightarrow G(Y, y)^{T, \text { Mal }}$.

In particular, there is a long exact sequence

$$
\begin{aligned}
\cdots \rightarrow \varpi_{n}(F, x)^{K, \text { Mal }} & \rightarrow \varpi_{n}(X, x)^{R, \text { Mal }} \rightarrow \varpi_{n}(Y, y)^{T, \text { Mal }} \rightarrow \varpi_{n-1}(F, x)^{K, \text { Mal }} \rightarrow \\
\cdots & \rightarrow \varpi_{1}(F, x)^{K, \text { Mal }} \rightarrow \varpi_{1}(X, x)^{R, \text { Mal }} \rightarrow \varpi_{1}(Y, y)^{T, \text { Mal }} \rightarrow 1 .
\end{aligned}
$$

Proof We adapt the proof of Theorem 2.43.

First observe that $\rho\left(\pi_{1}(F, x)\right)$ is normal in $\pi_{1}(X, x)$, so $K$ is normal in $R$, and $T$ is therefore a reductive pro-algebraic group, so $(Y, y)^{T, \text { Mal }}$ is well-defined. Next, observe that since $K$ is normal in $R, \mathrm{R}_{\mathrm{u}}(K)$ is also normal in $R$, and is therefore 1 , ensuring that $K$ is reductive, so $(F, x)^{K \text {,Mal }}$ is also well-defined.

Consider the complex $O(R) \otimes O(T) O\left(G(Y, y)^{T, \mathrm{Mal}}\right)$ of $G(X, x)^{R, \mathrm{Mal}}$-representations, regarded as a cosimplicial $G(X, x)$-representation. Since $G(F, x) \rightarrow \operatorname{ker}(G(X, x) \rightarrow$ $G(Y, y))$ is a weak equivalence, the Hochschild-Serre spectral sequence for $f$ (see Proposition 2.36) with coefficients in this complex is

$$
\begin{aligned}
E_{2}^{i, j}= & \mathbb{H}^{i}\left(G(Y, y), \mathrm{H}^{j}(F, O(R)) \otimes_{O(T)} O\left(G(Y, y)^{T, \mathrm{Mal}}\right)\right) \\
& \Longrightarrow \mathbb{H}^{i+j}\left(G(X, x), O(R) \otimes_{O(T)} O\left(G(Y, y)^{T, \mathrm{Mal}}\right)\right) .
\end{aligned}
$$


Regarding $O(R)$ as a $K$-representation, $\mathrm{H}^{*}(F, O(R))$ is a $\varpi_{1}(Y, y)^{T, \text { Mal }}$-representation by hypothesis. Hence $\mathrm{H}^{*}(F, O(R)) \otimes_{O(T)} O\left(G(Y, y)^{T, \text { Mal }}\right)$ is a cosimplicial $G(Y, y)^{T, \mathrm{Mal}}$-representation, so

$$
\begin{aligned}
& \mathbb{H}^{i}\left(G(Y, y), \mathrm{H}^{j}(F, O(R)) \otimes_{O(T)} O\left(G(Y, y)^{T, \mathrm{Mal}}\right)\right) \\
& \cong \mathbb{H}^{i}\left(G(Y, y)^{T, \mathrm{Mal}}, \mathrm{H}^{j}(F, O(R)) \otimes_{O(T)} O\left(G(Y, y)^{T, \mathrm{Mal}}\right)\right),
\end{aligned}
$$

by Lemma 2.42 .

Now, $\mathrm{H}^{*}(F, O(R)) \otimes O(T) O\left(G(Y, y)^{T, \mathrm{Mal}}\right)$ is a fibrant cosimplicial $G(Y, y)^{T, \mathrm{Mal}_{-}}$ representation, so

$$
\begin{aligned}
\mathbb{H}^{i}\left(G(Y, y)^{T, \mathrm{Mal}}, \mathrm{H}^{j}\right. & \left.(F, O(R)) \otimes O(T) O\left(G(Y, y)^{T, \mathrm{Mal}}\right)\right) \\
& \cong \mathrm{H}^{i} \Gamma\left(G(Y, y)^{T, \mathrm{Mal}}, \mathrm{H}^{j}(F, O(R)) \otimes O(T) O\left(G(Y, y)^{T, \mathrm{Mal}}\right)\right) \\
& = \begin{cases}\mathrm{H}^{j}(F, O(R)) \otimes_{O(T)} k=\mathrm{H}^{j}(F, O(K)) & i=0, \\
0 & i \neq 0,\end{cases}
\end{aligned}
$$

so

$$
\mathbb{H}^{j}\left(G(X, x), O(R) \otimes_{O(T)} O\left(G(Y, y)^{T, \mathrm{Mal}}\right)\right) \cong \mathrm{H}^{j}(F, O(K)) .
$$

Now, let $\mathcal{F}$ be the homotopy fibre of $G(X, x)^{R \text {,Mal }} \rightarrow G(Y, y)^{T \text {,Mal }}$ (which is just the kernel as this map is surjective), noting that there is a natural map $G(F, x)^{K \text {, Mal }} \rightarrow \mathcal{F}$. Lemma 2.42 implies that

$$
\begin{aligned}
& \mathbb{H}^{j}\left(G(X, x), O(R) \otimes_{O(T)} O\left(G(Y, y)^{T, \mathrm{Mal}}\right)\right) \\
& \quad=\mathbb{H}^{j}\left(G(X, x)^{R, \mathrm{Mal}}, O(R) \otimes_{O(T)} O\left(G(Y, y)^{T, \mathrm{Mal}}\right)\right),
\end{aligned}
$$

and [37, Theorem 1.51] gives a Hochschild-Serre spectral sequence

$$
\begin{aligned}
& \mathbb{H}^{i}\left(G(Y, y)^{T, \mathrm{Mal}}, \mathrm{H}^{j}(\mathcal{F}, O(R)) \otimes O(T) O\left(G(Y, y)^{T, \mathrm{Mal}}\right)\right) \\
& \Longrightarrow \mathbb{H}^{i+j}\left(G(X, x)^{R, \mathrm{Mal}}, O(R) \otimes_{O(T)} O\left(G(Y, y)^{T, \mathrm{Mal}}\right)\right) .
\end{aligned}
$$

The reasoning above adapts to show that this spectral sequence also collapses, yielding

$$
\mathrm{H}^{j}(\mathcal{F}, O(K))=\mathbb{H}^{j}\left(G(X, x), O(R) \otimes_{O(T)} O\left(G(Y, y)^{T, \mathrm{Mal}}\right)\right) .
$$

We have therefore shown that the map $G(F, x)^{K, \text { Mal }} \rightarrow \mathcal{F}$ gives an isomorphism

$$
\mathrm{H}^{*}(\mathcal{F}, O(K)) \rightarrow \mathrm{H}^{*}\left(G(F, x)^{K, \mathrm{Mal}}, O(K)\right),
$$

and hence isomorphisms $\mathrm{H}^{*}(\mathcal{F}, V) \rightarrow \mathrm{H}^{*}\left(G(F, x)^{K, \text { Mal }}, V\right)$ for all $K$-representations $V$. Since this is a morphism of simplicial pro-unipotent extensions of $K$, [37, Corollary 1.55] implies that $G(F, x)^{K \text {,Mal }} \rightarrow \mathcal{F}$ is a weak equivalence. 
Examples 3.33 Note that we can apply this theorem to $f_{\text {ét }}: X_{\text {ét }} \rightarrow Y_{\text {ét }}$ whenever $f: X \rightarrow Y$ is geometric fibration in the sense of [10, Definition 11.4]. This includes smooth projective morphisms, as well as smooth quasiprojective morphisms where the divisor is transverse to $f$. The fibre of $f_{\text {ét }}$ over $y$ will then be equivalent to $\left(f^{-1}\{y\}\right)_{\text {ét }}$.

Another source of examples comes from nerves of pro-finite groups. Any surjection $g: \Gamma \rightarrow \Delta$ of pro-finite groups gives a pro-fibration $B \Gamma \rightarrow B \Delta$, with fibre $B(\operatorname{ker} g)$. Of course, even if $f: X \rightarrow Y$ is not a pro-fibration, we can take a fibrant replacement. This will have connected fibres if and only if $\pi_{1}(X, x) \rightarrow \pi_{1}(Y, y)$ is surjective, and the theorem then describes the homotopy fibre of $f$.

\subsection{Comparison with Artin-Mazur homotopy groups}

Lemma 3.34 Let $f: X \rightarrow Y$ be a morphism in $\operatorname{pro}(\mathbb{S})_{\delta}$ for which the map

$$
\pi_{n}(f): \pi_{n}(X) \rightarrow \pi_{n}(Y)
$$

is a pro-isomorphism for $n \leq N$ and a pro-surjection for $n=N+1$, and take a continuous Zariski-dense morphism $\rho: \pi_{f} Y \rightarrow R\left(\mathbb{Q}_{\ell}\right)$. Then the map

$$
\varpi_{n}(f): \varpi_{n}(X, \rho \circ f)^{\mathrm{Mal}} \rightarrow \varpi_{n}(Y, \rho)^{\mathrm{Mal}}
$$

is an isomorphism for $n \leq N$ and a surjection for $n=N+1$.

Proof The proof of Lemma 2.22 carries over to this generality.

Definition 3.35 By analogy with Definition 2.23, say that a locally pro-discrete groupoid $\Gamma$ is $n$-good with respect to a continuous Zariski-dense representation $\rho: \Gamma \rightarrow R\left(\mathbb{Q}_{\ell}\right)$ to a reductive pro-algebraic groupoid if for all finite-dimensional $\Gamma^{\rho, \mathrm{Mal}}$-representations $V$, the map

$$
\mathrm{H}^{i}\left(\Gamma^{\rho, \mathrm{Mal}}, V\right) \rightarrow \mathrm{H}^{i}(\Gamma, V)
$$

is an isomorphism for all $i \leq n$ and an inclusion for $i=n+1$. Say that $\Gamma$ is $\operatorname{good}$ with respect to $\rho$ if it is $n-\operatorname{good}$ for all $n$.

If $\Gamma$ is $(n-) \operatorname{good}$ relative to $\Gamma^{\text {red }}$, then we say that $\Gamma$ is algebraically $(n-)$ good.

Lemma 3.36 A pro-groupoid $\Gamma$ is $N$-good with respect to $\rho$ if and only if for any finite-dimensional $\Gamma^{\rho, \mathrm{Mal}}$-representation $V$, and $\alpha \in \mathrm{H}^{n}(\Gamma, V)$ for $n \leq N$, there exists an injection $f: V \rightarrow W_{\alpha}$ of finite-dimensional $\Gamma^{\rho, \mathrm{Mal}}$-representations, with $f(\alpha)=0 \in \mathrm{H}^{n}\left(\Gamma, W_{\alpha}\right)$.

Proof This is a special case of the results of [41, Section 1.2.3], which adapt directly from groups to groupoids. 
Lemma 3.37 Let $\Gamma$ be a locally finitely presented $(L, N)$-good groupoid and let $\rho: \Gamma^{\wedge_{L}} \rightarrow R\left(\mathbb{Q}_{\ell}\right)$ be a Zariski-dense representation, with $\ell \in L$. Then $\Gamma$ is $N$-good relative to $\rho: \Gamma \rightarrow R\left(\mathbb{Q}_{\ell}\right)$ if and only if $\Gamma^{\wedge} L$ is $N-\operatorname{good}$ relative to $\rho$.

Proof Take a finite-dimensional $R$-representation $V$. By Lemma 3.22, $(B \Gamma)^{\rho, \text { Mal }} \simeq$ $(B \Gamma)^{L, \rho, \mathrm{Mal}}$. Since $\Gamma$ is $L$-good, Proposition 1.36 gives that $\pi_{n}\left((B \Gamma)^{\wedge} L\right)=0$ for all $1<n \leq N$. Applying Lemma 3.34 to the morphism $(B \Gamma)^{\wedge} L \rightarrow B\left(\Gamma^{\wedge} L\right)$, the observations above show that

$$
\varpi_{n}(B \Gamma)^{\rho, \mathrm{Mal}} \rightarrow \varpi_{n}\left(B\left(\Gamma^{\wedge} L\right)\right)^{L, \rho, \mathrm{Mal}}
$$

is an isomorphism for $n \leq N$ and a surjection for $n=N+1$.

Now, [41, Section 1.2.3] shows that a pro-group $G$ is $N$-good relative to $\rho$ if and only if $\varpi_{n}(B G)^{L, \rho, \mathrm{Mal}}=0$ for $1<n \leq N$, and the same proof adapts to groupoids. Thus $\Gamma$ is $N$-good relative to $\rho$ if and only if $\Gamma^{\wedge} L$ is so.

Examples 3.38 A pro-finite group $\Gamma$ is good with respect to a representation $\rho: \Gamma^{\wedge} L \rightarrow$ $R$ whenever any of the following holds:

(1) $\Gamma$ is finite, or $\Gamma^{\wedge_{L}} \cong \Delta^{\wedge_{L}}$, for $\Delta$ a finitely generated free discrete group.

(2) $\Gamma^{\wedge} L \cong \Delta^{\wedge} L$, for $\Delta$ a finitely generated nilpotent discrete group.

(3) $\Gamma^{\wedge_{L}} \cong \Delta^{\wedge} L$, for $\Delta$ the fundamental group of a compact Riemann surface. In particular, this applies if $\Gamma$ is the fundamental group of a smooth projective curve $C / k$, for $k$ a separably closed field whose characteristic is not in $L$.

(4) If $1 \rightarrow F \rightarrow \Gamma \rightarrow \Pi \rightarrow 1$ is an exact sequence of groups, with $F$ finite and $F^{\wedge_{L}} \rightarrow \Gamma^{\wedge_{L}}$ injective, assume that $\Pi^{\wedge_{L}}$ is good relative to $R / \overline{\rho(F)}$, where denotes Zariski closure. Then $\Gamma$ is good relative to $\rho$.

Proof Combine Lemma 3.37 with Examples 1.28 and [37, Examples 3.20].

Remark 3.39 For an example of an important pro-finite group which is not good with respect to a representation, note that $\operatorname{Sp}_{g}\left(\mathbb{Z}_{\ell}\right)$ is not good with respect to the natural map $\rho: \operatorname{Sp}_{g}\left(\mathbb{Z}_{\ell}\right) \rightarrow \operatorname{Sp}_{g}\left(\mathbb{Q}_{\ell}\right)$ for $g \geq 2$. In fact, $\varpi_{2}\left(\left(B \operatorname{Sp}_{g}\left(\mathbb{Z}_{\ell}\right)\right)^{\rho, \mathrm{Mal}}\right) \cong \mathbb{G}_{a}$. This issue arises in [14], considering the pro-finite mapping class group $\Gamma_{g}$ acting on a genus $g$ curve. The action on cohomology gives a map $\rho: \Gamma_{g} \rightarrow \operatorname{Sp}_{g}\left(\mathbb{Z}_{\ell}\right)$ with kernel $T_{g}$, the Torelli subgroup, and the map $T_{g}^{1 \text {,Mal }} \rightarrow \operatorname{ker}\left(\Gamma_{g}^{\rho, \text { Mal }} \rightarrow \mathrm{Sp}_{g}\right)$ has kernel $\mathbb{G}_{a}$. Theorem 3.32 allows us to interpret this copy of $\mathbb{G}_{a}$ as the image of the connecting homomorphism $\varpi_{2}\left(\left(B \operatorname{Sp}_{g}\left(\mathbb{Z}_{\ell}\right)\right)^{\rho, \text { Mal }}\right) \rightarrow T_{g}^{1, \mathrm{Mal}}$. 
Theorem 3.40 Let $L$ be a set of primes containing $\ell$, and take $X \in \operatorname{pro}(\mathbb{S})_{\delta}$ with fundamental groupoid $\pi_{f} X=\Gamma$, equipped with a continuous Zariski-dense representation $\rho: \Gamma^{\wedge_{L}} \rightarrow R\left(\mathbb{Q}_{\ell}\right)$ to a reductive pro-algebraic groupoid. If

(1) $\pi_{n}\left(X^{\wedge},-\right) \otimes_{\widehat{\mathbb{Z}}} \mathbb{Q}_{\ell}$ is finite-dimensional for all $1<n \leq N$, and

(2) the $\Gamma^{\wedge} L$-representation $\pi_{n}\left(X^{\wedge_{L}},-\right) \otimes_{\widehat{\mathbb{Z}}} \mathbb{Q}_{\ell}$ is an extension of $R$-representations (ie a $\Gamma^{L, \rho, \mathrm{Mal}}$-representation) for all $1<n \leq N$,

then for each $x \in X$ there is an exact sequence

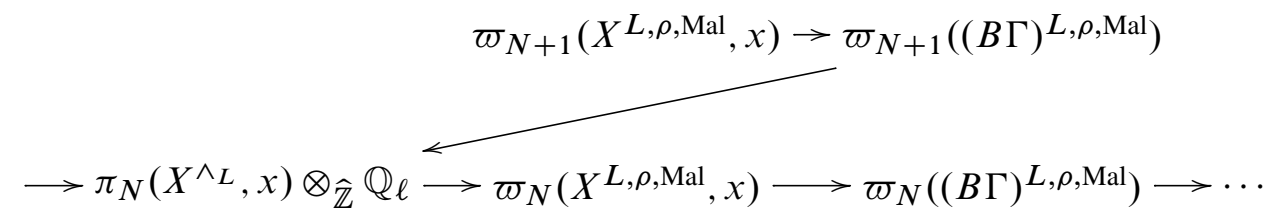

$\cdots \rightarrow \pi_{2}\left(X^{\wedge} L, x\right) \otimes_{\widehat{\mathbb{Z}}} \mathbb{Q}_{\ell} \longrightarrow \varpi_{2}\left(X^{L, \rho, \mathrm{Mal}}, x\right) \longrightarrow \varpi_{2}\left((B \Gamma)^{L, \rho, \mathrm{Mal}}\right) \longrightarrow 0$.

In particular, if in addition $\Gamma^{\wedge L}$ is $(N+1)$-good (resp. $N$-good) with respect to $\rho$, then the canonical map

$$
\pi_{n}\left(X^{\wedge},-\right) \otimes_{\widehat{\mathbb{Z}}} \mathbb{Q}_{\ell} \rightarrow \varpi_{n}\left(X^{L, \rho, \mathrm{Mal}}\right)
$$

is an isomorphism for all $n \leq N$ (resp. an isomorphism for all $n<N$ and a surjection for $n=N)$.

Proof Without loss of generality, we may assume that $X$ is connected, choose a point $x \in X$, and replace $R$ with the group $R(x, x)$. Let $(\tilde{X}, x)$ be the universal cover of $(X, x)$, and note that we have a homotopy fibration sequence $(\tilde{X}, x) \rightarrow$ $(X, x) \rightarrow B \pi_{1}(X, x)$, which means that we can apply Theorem 3.32 (after taking a fibrant replacement for $\left.(X, x) \rightarrow B \pi_{1}(X, x)\right)$. This immediately gives the long exact sequence

$$
\begin{aligned}
\cdots \rightarrow \varpi_{n}(\tilde{X}, x) & \rightarrow \varpi_{n}(X, x)^{R, \text { Mal }} \rightarrow \varpi_{n}\left(B \pi_{1}(X, x)\right)^{R, \text { Mal }} \rightarrow \varpi_{n-1}(\tilde{X}, x)^{K, \text { Mal }} \\
& \rightarrow \cdots \rightarrow \varpi_{2}(\tilde{X}, x) \rightarrow \varpi_{2}(X, x)^{R, \text { Mal }} \rightarrow \varpi_{2}\left(B \pi_{1}(X, x)\right)^{R, \text { Mal }} \rightarrow 0 .
\end{aligned}
$$

It therefore suffices to show that

$$
\pi_{n}\left(\tilde{X}^{\wedge}, x\right) \otimes_{\widehat{\mathbb{Z}}} \mathbb{Q}_{\ell} \rightarrow \varpi_{n}\left(\tilde{X}^{L, \mathrm{alg}}, x\right)
$$

is an isomorphism for $n \leq N$. 
We may assume that $\tilde{X}=\left\{\tilde{X}_{\alpha}\right\}_{\alpha}$ is an inverse system of fibrant simplicial sets, and then form the tower $\{\tilde{X}(n)\}_{n}$ by setting $\tilde{X}(n)=\left\{\tilde{X}_{\alpha}(n)\right\}_{\alpha}$, where $\left\{\tilde{X}_{\alpha}(n)\right\}_{n}$ the Moore-Postnikov tower of $\widetilde{X}_{\alpha}$.

Note that if $\tilde{X}(N)$ satisfies the theorem, then we can apply Lemma 3.34 to the morphism $\tilde{X} \rightarrow \tilde{X}(N)$, so $\tilde{X}$ will also satisfy the theorem. We now prove by induction on $n$ that $\tilde{X}(n)$ satisfies the theorem for $n \leq N$.

For $n=1, \tilde{X}(1)$ is contractible, making the long exact sequence automatic. Now, assume that $\tilde{X}(n-1)$ satisfies the inductive hypothesis, and consider the pro-fibration $\tilde{X}(n) \rightarrow \tilde{X}(n-1)$, with fibre $E(n)$ over $x$. Properties of the Postnikov tower give that $\pi_{i} \tilde{X}(n)=\pi_{i} \tilde{X}$ for all $i \leq n$, with $E(n)$ being a $K\left(\pi_{n} X, n\right)$-space.

The long exact sequence of Theorem 3.32 gives $\varpi_{i}\left(E(n)^{\text {alg }}\right) \cong \varpi_{i}\left(\tilde{X}(n)^{L, \rho, \text { Mal }}\right)$ for $i \geq n$, and exact sequences

$$
\varpi_{i}\left(E(n)^{\mathrm{alg}}\right) \rightarrow \varpi_{i}\left(\tilde{X}(n)^{L, \mathrm{alg}}\right) \rightarrow \pi_{i}\left(\tilde{X}^{\wedge L}\right) \otimes_{\widehat{\mathbb{Z}}} \mathbb{Q}_{\ell} \rightarrow \varpi_{i-1}\left(E(n)^{\mathrm{alg}}\right) .
$$

Since $E(n)$ is a $K\left(\pi_{n} X, n\right)$-space, the problem thus reduces to establishing the theorem for the case when $X$ is a $K(\pi, n)$ space (for $n \geq 2$ ), and $R=1$. Unlike [37, Theorem 1.58], we cannot now immediately appeal to the Curtis convergence theorem to show that for any pro-discrete abelian group $\pi$ and $n \geq 2$, the map

$$
G(K(\pi, n))^{L, \text { alg }} \rightarrow N^{-1}\left(\hat{\pi} \otimes_{\widehat{\mathbb{Z}}} \mathbb{Q}_{\ell}[1-n]\right)
$$

is a weak equivalence of simplicial unipotent groups.

Instead, observe that we may replace $\pi$ by $\pi^{\hat{\ell}}$, so assume that $\pi$ is a pro- $\ell$ group. Since $\pi \otimes_{\mathbb{Z}_{\ell}} \mathbb{Q}_{\ell}$ is finite-dimensional, we may write $\pi=v \hat{l}$, for $v$ an abelian group of finite rank. On cohomology, we have maps

$$
\mathrm{H}^{*}\left(N^{-1}\left(\pi \otimes_{\mathbb{Z}_{\ell}} \mathbb{Q}_{\ell}[1-n]\right), \mathbb{Q}_{\ell}\right) \rightarrow \mathrm{H}^{*}\left(K(\pi, n), \mathbb{Q}_{\ell}\right) \rightarrow \mathrm{H}^{*}\left(K(\nu, n), \mathbb{Q}_{\ell}\right) .
$$

By [44, Theorem I.3.4], the Lie algebra $v \otimes_{\mathbb{Z}} \mathbb{Q}_{\ell}[1-n]$ is the $\mathbb{Q}_{\ell}$-homotopy type of $K(v, n)$. Since $\pi \otimes_{\mathbb{Z}_{\ell}} \mathbb{Q}_{\ell}=v \otimes_{\mathbb{Z}} \mathbb{Q}_{\ell}$, the composite is an isomorphism in $(\dagger)$, while the second map is an isomorphism by Lemma 1.35. Thus the first map is also an isomorphism, as required.

For the final part, we just note that [41, Section 1.2.3] shows that $\Gamma$ is $N$-good relative to $\rho$ if and only if $\varpi_{n}\left((B \Gamma)^{L, \rho, \mathrm{Mal}}\right)=0$ for $1<n \leq N$.

\subsection{Comparison of homotopy types for complex varieties}

Let $X \bullet$ be a simplicial scheme of finite type over $\mathbb{C}$. To this we may associate the étale homotopy type $X_{\text {ét }} \in \operatorname{pro}(\mathbb{S}$ ) (as in Example 3.18). There is also an analytic homotopy 
type $X_{\text {an }}:=\operatorname{diag} \operatorname{Sing}\left(X_{\bullet}(\mathbb{C})\right) \in \mathbb{S}$, where diag is the diagonal functor on bisimplicial sets. We now compare the corresponding schematic homotopy types.

Lemma 3.41 If $G$ is a pro-algebraic group over $\mathbb{Q}_{\ell}$, and $\rho: \pi_{f}\left(X_{\mathrm{an}}\right) \rightarrow G\left(\mathbb{Q}_{\ell}\right)$ a representation with compact image (for the $\ell$-adic topology on $G\left(\mathbb{Q}_{\ell}\right)$ ), then $\rho$ factorises canonically through $\widehat{\pi_{f}\left(X_{\mathrm{e}}\right)}$, giving a continuous representation

$$
\rho: \widehat{\pi_{f}\left(X_{\text {et }}\right)} \rightarrow G\left(\mathbb{Q}_{\ell}\right) .
$$

Proof It follows from [10, Theorem 8.4] that

$$
\widehat{\pi_{f}\left(X_{\mathrm{et}}\right)} \cong \widehat{\pi_{f}\left(X_{\mathrm{an}}\right)} .
$$

Since $G\left(\mathbb{Q}_{\ell}\right)$ is totally disconnected, any compact subgroup is pro-finite, completing the proof.

Now, given a reductive pro-algebraic groupoid $R$, and $\rho: \pi_{f}\left(X_{\mathbb{C}}\right) \rightarrow R\left(\mathbb{Q}_{\ell}\right)$ with compact Zariski-dense image, we may compare the relative Malcev homotopy type $X_{\mathrm{an}}^{\rho \text {,Mal }}$ of [37, Definition 3.16] with the relative Malcev homotopy type $X_{\mathrm{et}}^{\rho, \text { Mal }}$ of Definition 3.20, since both are objects of $\operatorname{Ho}(s \mathcal{E}(R))$.

Theorem 3.42 For $X, \rho$ as above, there is a canonical isomorphism

$$
X_{\mathrm{an}}^{\rho, \mathrm{Mal}} \cong X_{\mathrm{et}}^{\rho, \mathrm{Mal}}
$$

Proof We adapt [10, Theorem 8.4], which constructs a new homotopy type $X_{\text {s.ét }}$, and gives morphisms

$$
X_{\text {ét }} \leftarrow X_{\text {s.ét }} \rightarrow X_{\text {an }}
$$

in $\operatorname{pro}(\mathbb{S})_{\delta}$, inducing weak equivalences on pro-finite completions. By Lemma 3.22, $X_{\text {an }}^{\rho, \text { Mal }}$ is quasi-isomorphic to $\widehat{X_{\text {an }}} \rho$, Mal . By Lemma 1.37, the maps

$$
\widehat{X_{\text {ét }}} \leftarrow \widehat{X_{\text {s.ét }}} \rightarrow \widehat{X_{\text {an }}}
$$

are weak equivalences in $\widehat{\mathbb{S}}$. Lemma 3.34 then implies that the maps

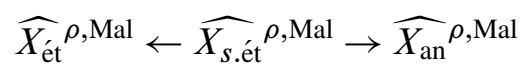

are quasi-isomorphisms, as required.

Remarks 3.43 In particular, this shows that there is an action of the Galois group $\operatorname{Gal}(\mathbb{C} / K)$ on the relative Malcev homotopy groups $\varpi_{n}\left(X_{\mathrm{an}}^{\rho, \mathrm{Mal}}\right)$ whenever $X$ is defined over a number field $K$ and $\rho$ is Galois-equivariant. The question of when this action is continuous will be addressed in Section 5 . 
It seems possible that the conditions of Theorem 2.25 might be satisfied in some cases where those of Theorem 3.40 do not hold, giving $\varpi_{n}\left(X_{\text {an }}^{\rho, \text { Mal }}\right) \cong \pi_{n}\left(X_{\text {an }}\right) \otimes_{\mathbb{Z}} \mathbb{Q}_{\ell}$, but no such examples are known to the author.

\section{Relative and filtered homotopy types}

The aims of this section are twofold. Firstly, we adapt some of the framework of pro-algebraic homotopy types to work over a base ring, rather than a base field. This is motivated by the need in Section 7.2 to phrase the étale-crystalline comparison over variants of Fontaine's ring $B_{\text {cris }}$ of $p$-adic periods, rather than just over $\mathbb{Q}_{p}$. Secondly, Section 4.3 develops techniques for transferring filtrations systematically from cochains to homotopy types. These will be used in Sections 6 and 7 to determine the structure of homotopy types of quasiprojective varieties. This is possible because the Gysin filtration on homotopy groups (unlike that on cohomology) is not determined by weights of Frobenius, so imposes further restrictions.

\subsection{Actions on pro-algebraic homotopy types}

Fix a $\mathbb{Q}_{\ell}$-algebra $A$, and a reductive pro-algebraic groupoid $R$ over $\mathbb{Q}_{\ell}$.

Definition 4.1 Define $c \operatorname{Alg}_{A}(R)$ (resp. $D G \operatorname{Alg}_{A}(R)$ ) to be the comma category $A \downarrow c \operatorname{Alg}(R)$ (resp. $A \downarrow D G \operatorname{Alg}(R)$ ), with model structure induced by Proposition 2.60 (resp. Lemma 2.68). Denote the opposite category by $s \operatorname{Aff}_{A}(R)$ (resp. $d g \operatorname{Aff}_{A}(R)$ ). Likewise, define

$$
\begin{aligned}
c \operatorname{Alg}_{A}(R)_{*} & :=c \operatorname{Alg}_{A}(R) \downarrow \prod_{x \in \mathrm{Ob} R} A \otimes O(R)(x,-), \\
D G \operatorname{Alg}_{A}(R)_{*} & :=D G \operatorname{Alg}_{A}(R) \downarrow \prod_{x \in \mathrm{Ob} R} A \otimes O(R)(x,-),
\end{aligned}
$$

and so on.

Observe that the Quillen equivalence of Proposition 2.70 induces Quillen equivalences between $d g \operatorname{Aff}_{A}(R)_{*}$ and $s \operatorname{Aff}_{A}(R)_{*}$, so gives the following equivalence of categories:

$$
\operatorname{Ho}\left(d g \operatorname{Aff}_{A}(R)_{*}\right) \stackrel{\operatorname{Spec} D}{\underset{\mathbf{R}\left(\operatorname{Spec} D^{*}\right)}{\longleftrightarrow}} H o\left(s \operatorname{Aff}_{A}(R)_{*}\right) .
$$

Although we do not have a precise analogue of Theorem 2.74 for $\operatorname{Ho}\left(d g \operatorname{Aff}_{A}(R)_{*}\right)_{0}$, we have the following: 
Lemma 4.2 Given $X \in d g \operatorname{Aff}(R)_{0 *}$ and $\mathfrak{g} \in d g \hat{\mathcal{N}}(R)$,

$$
\begin{aligned}
& \operatorname{Hom}_{\mathrm{Ho}\left(d g \operatorname{Aff}_{A}(R)_{*}\right)}(X \otimes A, \bar{W} \mathfrak{g} \otimes A) \\
\cong & \operatorname{Hom}_{\mathrm{Ho}\left(d g \hat{\mathcal{N}}_{A}(R)\right)}(\bar{G}(X) \hat{\otimes} A, \mathfrak{g} \hat{\otimes} A) \times^{\exp \left(\mathfrak{g}_{0}^{R} \hat{\otimes} A\right)} \prod_{x \in \mathrm{Ob} R} \exp \left(\mathrm{H}_{0} \mathfrak{g}(x) \hat{\otimes} A\right) .
\end{aligned}
$$

Proof The proof of [37, Proposition 3.48] adapts to this context.

\subsection{Homotopy actions}

Definition 4.3 Given $\mathfrak{g} \in s \widehat{\mathcal{P}}_{R}$, define a group-valued functor $\operatorname{Aut}_{R}(\mathfrak{g})$ on the category of $\mathbb{Q}_{\ell}$-algebras by setting

$$
\operatorname{Aut}_{R}(\mathfrak{g})(A):=\operatorname{Aut}_{S \mathcal{P}_{A}(R)}(\mathfrak{g} \hat{\otimes} A) .
$$

Given $G \in s \mathcal{E}(R)$, define $\operatorname{RAut}(G):=\operatorname{Aut}_{R}\left(\mathrm{R}_{\mathrm{u}}(G)\right)$, noting that

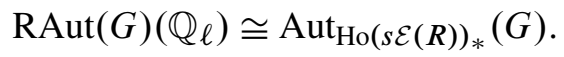

For $G \in s \operatorname{AGpd}$, set $\operatorname{RAut}(G):=\operatorname{Aut}_{G^{\mathrm{red}}}\left(\mathrm{R}_{\mathrm{u}}(G)\right)$.

Lemma 4.4 If $G \in s \mathcal{E}(R)$ is such that $\mathrm{H}^{i}(G, V)$ is finite-dimensional for all $i$ and all finite-dimensional irreducible $R$-representations $V$, then the group-valued functor

$$
\operatorname{RAut}(G)
$$

is represented by a pro-algebraic group over $\mathbb{Q}_{\ell}$. The map

$$
\operatorname{RAut}(G) \rightarrow \prod_{x \in \mathrm{Ob} R} \prod_{i} \operatorname{Aut}_{R}\left(\mathrm{H}^{i}(G, O(R)(x,-))\right)
$$

of pro-algebraic groups has pro-unipotent kernel.

Proof This is a consequence of [37, Theorem 5.13], which proves the corresponding statement for the group $\operatorname{ROut}(G):=\operatorname{RAut}(G) / \prod_{x \in \mathrm{Ob} G} \mathrm{R}_{\mathrm{u}}(G)(x)$. Since the group $\prod_{x \in \mathrm{Ob} G} \mathrm{R}_{\mathrm{u}}(G)(x)$ is pro-unipotent pro-algebraic, the result follows.

Definition 4.5 Given a pro-algebraic groupoid $G$, we may extend the automorphism group $\operatorname{Aut}(G)$ to a group presheaf over $\mathbb{Q}_{\ell}$, by setting

$$
\operatorname{Aut}(G)(A):=\operatorname{Aut}_{A}\left(G \times \times_{\operatorname{Spec}} \mathbb{Q}_{\ell} \operatorname{Spec} A\right) .
$$


Lemma 4.6 For $G \in s \mathcal{E}(R)$, there is a group presheaf $\operatorname{Aut}^{h}(G)$ over $\mathbb{Q}_{\ell}$, with the properties that $\operatorname{Aut}^{h}(G)\left(\mathbb{Q}_{\ell}\right)$ is the group of automorphisms of $G$ in $\mathrm{Ho}(\mathrm{Ob} G \downarrow s \mathrm{AGpd})$, and that there is an exact sequence

$$
1 \rightarrow \operatorname{RAut}(G) \rightarrow \operatorname{Aut}^{h}(G) \stackrel{\alpha}{\rightarrow} \operatorname{Aut}(R) \rightarrow 1,
$$

where $\operatorname{Aut}(R)$ is given the algebraic structure of Definition 4.5.

If $\mathrm{H}^{i}(G, V)$ is finite-dimensional for all $i$ and all finite-dimensional irreducible $R$ representations $V$, then $\alpha$ is fibred in affine schemes.

Proof Let $R=G^{\text {red }}$, take $Y \in \operatorname{Ho}\left(d g \operatorname{Aff}(R)_{*}\right)$ corresponding to $G$ under the equivalence of Theorem 2.74 and define $\operatorname{Aut}^{h}(G)(A):=\left\{(f, \theta): f \in \operatorname{Aut}(R)(A), \theta \in \operatorname{Iso}_{H o}\left(d g \operatorname{Aff}_{A}(R)_{*}\right)\left(Y \otimes A, f^{\sharp} Y \otimes A\right)\right\}$.

We may now take a minimal model $\mathfrak{m}$ for $\bar{G}(Y) \in d g \hat{\mathcal{N}}(R)$, and observe that Lemma 4.2 then gives

$$
\begin{aligned}
& \operatorname{Hom}_{\mathrm{Ho}\left(d g \operatorname{Aff}_{A}(R)_{*}\right)}\left(Y \otimes A, f^{\sharp} Y \otimes A\right) \\
\cong & \operatorname{Hom}_{\mathrm{Ho}\left(d g \operatorname{Aff}_{A}(R)_{*}\right)}\left(Y \otimes A, f^{\sharp} \bar{W} \mathfrak{m} \otimes A\right) \\
\cong & \operatorname{Hom}_{\mathrm{Ho}\left(d g \hat{\mathcal{N}}_{A}(R)_{*}\right)}(\bar{G}(Y) \hat{\otimes} A, \mathfrak{m} \hat{\otimes} A) \times^{\exp \left(\mathfrak{m}_{0}^{R} \hat{\otimes} A\right)} \prod_{x \in \mathrm{Ob} R} \exp \left(\mathrm{H}_{0} \mathfrak{m}(x) \hat{\otimes} A\right) \\
\cong & \operatorname{Hom}_{\mathrm{Ho}\left(d g \hat{\mathcal{N}}_{A}(R)\right)}(\mathfrak{m} \hat{\otimes} A, \mathfrak{m} \hat{\otimes} A) \times^{\exp \left(\mathfrak{m}_{0}^{R} \hat{\otimes} A\right)} \prod_{x \in \mathrm{Ob} R} \exp \left(\mathrm{H}_{0} \mathfrak{m}(x) \hat{\otimes} A\right) .
\end{aligned}
$$

The proof that $\alpha$ is fibred in affine schemes is now essentially the same as Lemma 4.4, which deals with the fibre over $1 \in \operatorname{Aut}(R)$.

Definition 4.7 Given a pro-discrete group $\Gamma$, we say a morphism $\Gamma \rightarrow \operatorname{Aut}^{h}(G)\left(\mathbb{Q}_{\ell}\right)$ is algebraic if it factors through a morphism $\Gamma^{\mathrm{alg}} \rightarrow \mathrm{Aut}^{h}(G)$ of presheaves of groups.

Corollary 4.8 If $\mathrm{H}^{i}(G, V)$ is finite-dimensional for all $i$ and all finite-dimensional irreducible $R$-representations $V$, then a morphism $\Gamma \rightarrow \operatorname{Aut}^{h}(G)\left(\mathbb{Q}_{\ell}\right)$ is algebraic whenever $\Gamma \rightarrow \operatorname{Aut}\left(G^{\text {red }}\right)$ is so.

Proof We have $\Gamma^{\mathrm{alg}} \rightarrow \operatorname{Aut}\left(G^{\mathrm{red}}\right)$, so $\theta: \Gamma \rightarrow\left(\Gamma^{\mathrm{alg}} \times_{\operatorname{Aut}\left(G^{\mathrm{red}}\right)} \operatorname{Aut}^{h}(G)\right)\left(\mathbb{Q}_{\ell}\right)$. Since $\operatorname{Aut}^{h}(G) \rightarrow \operatorname{Aut}\left(G^{\text {red }}\right)$ is fibred in affine schemes, the group on the right is pro-algebraic, so $\theta$ factors through $\Gamma^{\mathrm{alg}}$, as required. 
If $R=G^{\text {red }}$, observe that there is canonical action of $\operatorname{Aut}^{h}(G)$ on the direct sum $\bigoplus_{x \in \mathrm{Ob} R} \mathrm{H}^{*}(G, O(R)(x,-))$. In fact, we have a homomorphism

$$
\beta: \operatorname{Aut}^{h}(G) \rightarrow \operatorname{Aut}(R) \times \operatorname{Aut}\left(\bigoplus_{x \in \mathrm{Ob} R} \mathrm{H}^{*}(G, O(R)(x,-))\right)
$$

of presheaves of groups.

Lemma 4.9 If $\mathrm{H}^{i}(G, V)$ is finite-dimensional for all $i$ and all finite-dimensional irreducible $R$-representations $V$, then the kernel of $\beta$ is a pro-unipotent pro-algebraic group.

Proof The kernel of $\beta$ is just the kernel of

$$
\operatorname{RAut}(G) \rightarrow \prod_{x \in \mathrm{Ob} R} \prod_{i} \operatorname{Aut}_{R} \mathrm{H}^{i}(G, O(R)(x,-)),
$$

which is pro-unipotent by Lemma 4.4.

\subsection{Filtered homotopy types}

\subsubsection{Commutative algebras}

Definition 4.10 Given a $\mathbb{Q}_{\ell}$-algebra $A$ and a reductive pro-algebraic groupoid $R$ over $\mathbb{Q}_{\ell}$, define $F D G \operatorname{Alg}_{A}(R)$ (resp. $F c \operatorname{Alg}_{A}(R)$ ) to consist of $R$-representations $B$ in nonnegatively graded cochain (resp. cosimplicial) algebras over $A$, equipped with an increasing exhaustive filtration $J_{0} B \subset J_{1} B \subset \cdots$ of $B$ as a DG (resp. cosimplicial) $(R, A)$-module, with the property that $\left(J_{m} B\right) \cdot\left(J_{n} B\right) \subset J_{m+n} B$. Morphisms are required to respect the filtration, and we assume that $1 \in J_{0} B$.

Write

$$
\begin{gathered}
F c \operatorname{Alg}(R)_{*}:=F c \operatorname{Alg}(R) \downarrow \prod_{x \in \mathrm{Ob} R} O(R)(x,-), \\
F D G \operatorname{Alg}(R)_{*}:=F D G \operatorname{Alg}(R) \downarrow \prod_{x \in \mathrm{Ob} R} O(R)(x,-),
\end{gathered}
$$

where $O(R)(x,-)=J_{0} O(R)(x,-)$.

Given $(B, J) \in F D G \operatorname{Alg}_{A}(R)$ or $F c \operatorname{Alg}_{A}(R)$, there is a spectral sequence ${ }_{J} \mathrm{E}_{*}^{*, *}(B)$ associated to the filtration $J$, with

$$
{ }_{J} \mathrm{E}_{1}^{a, b}(B)=\mathrm{H}^{a+b}\left(\mathrm{Gr}_{-a}^{J} B\right) .
$$


Definition 4.11 We regard ${ }_{J} \mathrm{E}_{1}^{*, *}(B)$ as an object of $F D G \operatorname{Alg}_{A}(R)$, with

$$
J_{m}\left({ }_{J} \mathrm{E}_{1}^{*, *}(B)\right)^{n}=\bigoplus_{r \leq m}{ }_{J} \mathrm{E}_{1}^{-r, n+r}(B),
$$

noting that $d\left(J_{m}\left(E_{1}\right)^{n}\right) \subset J_{m-1}\left(E_{1}\right)^{n+1}$.

Definition 4.12 A map $f: B \rightarrow C$ is a fibration if the maps $J_{n} f: J_{n} B \rightarrow J_{n} C$ are all surjective. A map $f$ is a weak equivalence if the maps ${ }_{J} \mathrm{E}_{1}^{*, *}(f):{ }_{J} \mathrm{E}_{1}^{*, *}(B) \rightarrow$ ${ }_{J} \mathrm{E}_{1}^{*, *}(C)$ are all isomorphisms.

Lemma 4.13 There are cofibrantly generated model structures on the categories $F c \operatorname{Mod}_{A}(R)$ and $F D G \operatorname{Mod}_{A}(R)$, with the classes of fibrations and weak equivalences above.

Proof First, normalisation gives an equivalence $F c \operatorname{Mod}_{A}(R) \rightarrow F D G \operatorname{Mod}_{A}(R)$ of categories, preserving and reflecting fibrations and weak equivalences. It thus suffices only to consider $F D G \operatorname{Mod}_{A}(R)$

Let $S_{n, m}$ denote the cochain complex consisting of $A$ concentrated in degree $n$, with $J_{m} S_{n, m}=S_{n, m}$ and $J_{m-1} S_{n, m}=0$. Let $D_{n, m}$ denote the cochain complex consisting of $A$ concentrated in degrees $n, n-1$ with differential $d^{n-1}$ the identity, $J_{m} D_{n, m}=D_{n, m}$ and $J_{m-1} D_{n, m}=0$. By convention, $D_{0, m}=0$. Note that there are natural maps $S_{n, m} \rightarrow D_{n, m}$.

For a set $\{V\}$ of representatives of irreducible $R$-representations in $\mathbb{Q}_{\ell}$-vector spaces, define $I$ to be the set of morphisms $A \otimes S_{n, m} \otimes V \rightarrow A \otimes D_{n, m} \otimes V$, for $n \geq 0$. Define $J$ to be the set of morphisms $0 \rightarrow A \otimes D_{n, m} \otimes V$, for $n \geq 0$.

Now

$$
\begin{aligned}
& \operatorname{Hom}_{F D G} \operatorname{Mod}_{A}(R)\left(A \otimes S_{n, m} \otimes V, M\right)=\operatorname{Hom}_{R}\left(V, J_{m} \mathrm{Z}^{n} M\right) \\
& \operatorname{Hom}_{F D G \operatorname{Mod}_{A}(R)}\left(A \otimes D_{n, m} \otimes V, M\right)=\operatorname{Hom}_{R}\left(V, J_{m} M^{n-1}\right),
\end{aligned}
$$

so a map $f: M \rightarrow N$ in $F D G \operatorname{Mod}_{A}(R)$ is then $I$-injective when

$$
J_{m} M^{n-1} \stackrel{f, d}{\longrightarrow} J_{m} N^{n-1} \times_{d, J_{m} \mathrm{Z}^{n} N, f} \mathrm{Z}^{n} M
$$

is surjective, for all $m, n$, and $J$-injective when

$$
J_{m} M^{n-1} \stackrel{f}{\rightarrow} J_{m} N^{n-1}
$$

is surjective for all $n$. Thus $I$-injectives are trivial fibrations, and $J$-injectives are fibrations. 
Since $S_{n, m}=D_{n+1, m} / S_{n+1, m}$, the map $0 \rightarrow D_{n, m}$ is a composition $0 \rightarrow S_{n, m} \rightarrow D_{n, m}$ of pushouts of maps in $I$, so maps in $J$ are all $I$-cofibrations. Since maps in $J$ are all weak equivalences, we have satisfied the conditions of [18, Theorem 2.1.19,] giving the model structure claimed.

Lemma 4.14 In the category $F D G \operatorname{Mod}(R)=F D G \operatorname{Mod}_{\mathbb{Q}_{\ell}}(R)$, all objects $V$ are cofibrant.

Proof Given $V \in F D G \operatorname{Mod}_{\mathbb{Q}_{\ell}}(R)$, it will suffice to show that $J_{0} V$ is cofibrant, and that all the maps $J_{m-1} V \rightarrow J_{m} V$ are cofibrations, since $V=\lim J_{m} V$. To do this, we will show that these maps are transfinite compositions of pushouts of generating cofibrations.

Now, since all $R$-representations are semisimple, we may choose decompositions $\operatorname{gr}_{m}^{J} V^{n}=M^{n} \oplus N^{n} \oplus d N^{n-1}$, with $d M^{n}=0$. By semisimplicity, we may also lift the $R$-modules $M^{i}, N^{i}$ to $\widetilde{M}^{i}, \tilde{N}^{i} \subset J_{m} V$. Now $d \widetilde{M} \subset J_{m-1} V$, so the map $J_{m-1} V \rightarrow J_{m} V$ is a pushout of $\bigoplus_{n}\left(S_{n+1, m} \otimes \widetilde{M}^{n}\right) \rightarrow \bigoplus_{n}\left(D_{n+1, m} \otimes \widetilde{M}^{n}\right) \oplus$ $\bigoplus_{n}\left(D_{n+1, m} \otimes \tilde{N}^{n}\right)$, and hence a cofibration. Since this argument also applies to $0 \rightarrow J_{0} V$, we deduce that $V$ is cofibrant.

Proposition 4.15 There is a cofibrantly generated model structure on $F D G \operatorname{Alg}_{A}(R)$ (resp. $F c \operatorname{Alg}_{A}(R)$ ), for which a morphism is a fibration or weak equivalence whenever the underlying morphism in $F D G \operatorname{Mod}_{A}(R)\left(\operatorname{resp} . F_{C} \operatorname{Mod}_{A}(R)\right)$ is so (in the model structure of Lemma 4.13).

Proof The forgetful functor $F D G \operatorname{Alg}_{A}(R) \rightarrow F D G \operatorname{Mod}_{A}(R)$ (resp. $F c \operatorname{Alg}_{A}(R) \rightarrow$ $\left.F c \operatorname{Mod}_{A}(R)\right)$ preserves filtered colimits and has a left adjoint, the free algebra functor. Since the free algebra functor maps trivial generating cofibrations to weak equivalences, we may apply [16, Theorem 11.3.2], which gives the required cofibrantly generated model structure. The generating cofibrations and trivial cofibrations are given by the images under the free algebra functor of the generating cofibrations and trivial cofibrations in $F D G \operatorname{Mod}_{A}(R)\left(\operatorname{resp} . F c \operatorname{Mod}_{A}(R)\right)$.

\subsubsection{Lie algebras}

Definition 4.16 Define $F \widehat{\mathcal{N}}_{A}(R)$ to be opposite to the category $F \widehat{\mathcal{N}}_{A}(R)^{\text {opp }}$ of $R-$ representations in ind-conilpotent (see Definition 2.44) Lie coalgebras $C$ over $A$, equipped with an exhaustive increasing filtration $J_{0} C \subset J_{1} C \subset \cdots$, of $C$ as an $(R, A)$-module, with the property that $\nabla\left(J_{r} C\right) \subset \sum_{m+n=r}\left(J_{m} C\right) \otimes\left(J_{n} C\right)$, for $\nabla$ the cobracket. Morphisms are required to respect the filtration. 
Similarly, $F d g \hat{\mathcal{N}}_{A}(R)$ is opposite to the category of $R$-representations in nonnegatively filtered ind-conilpotent $\mathbb{N}_{0}$-graded cochain Lie coalgebras over $A$. Fs $\hat{\mathcal{N}}_{A}(R)$ is the category of simplicial objects in $F \hat{\mathcal{N}}_{A}(R)$. When $A=\mathbb{Q}_{\ell}$, we will usually drop the subscript $A$.

Proposition 4.17 There is a closed model structure on $F d g \widehat{\mathcal{N}}_{A}(R)\left(\right.$ resp. $\left.F s \widehat{\mathcal{N}}_{A}(R)\right)$, in which a morphism $f: \mathfrak{g} \rightarrow \mathfrak{h}$ is a fibration or a weak equivalence whenever the underlying map $f^{\vee}: \mathfrak{h}^{\vee} \rightarrow \mathfrak{g}^{\vee}$ in $F D G \operatorname{Mod}_{A}(R)\left(\operatorname{resp} . F c \operatorname{Mod}_{A}(R)\right)$ is a cofibration or a weak equivalence.

Proof The proof of [37, Lemma 5.9] carries over to this context.

\subsubsection{Equivalences}

Definition 4.18 Define $F c \operatorname{Alg}(R)_{00_{*}}$ (respectively, $F D G \operatorname{Alg}(R)_{00_{*}}$ ) to be the full subcategory of $F c \operatorname{Alg}_{A}(R)_{*}$ (respectively, $\left.F D G \operatorname{Alg}_{A}(R)_{*}\right)$ consisting of objects $B$ with $B^{0}=\mathbb{Q}_{\ell}$. Let $F c \operatorname{Alg}(R)_{0 *}$ (respectively, $F D G \operatorname{Alg}(R)_{0_{*}}$ ) be the full subcategory consisting of objects weakly equivalent to objects of $F c \operatorname{Alg}(R)_{00_{*}}$ (respectively, $\left.F D G \operatorname{Alg}(R)_{00_{*}}\right)$. Define $\operatorname{Ho}\left(F c \operatorname{Alg}(R)_{*}\right)_{0}$ (respectively, $\left.\operatorname{Ho}\left(F D G \operatorname{Alg}(R)_{*}\right)_{0}\right)$ to be the full subcategory of $\operatorname{Ho}\left(F c \operatorname{Alg}(R)_{*}\right)$ (respectively, $\operatorname{Ho}\left(F D G \operatorname{Alg}(R)_{*}\right)$ ) on objects $F c \operatorname{Alg}(R)_{0_{*}}$ (respectively, $F D G \operatorname{Alg}(R)_{0_{*}}$ ). Denote the opposite category to $F c \operatorname{Alg}(R)_{00 *}$ by $F s \operatorname{Aff}(R)_{00 *}$, etc.

Definition 4.19 Given $\mathfrak{g} \in F s \hat{\mathcal{N}}(R)$, we define $\bar{W} \mathfrak{g} \in F s \operatorname{Aff}(R)$ by

$$
(\bar{W} \mathfrak{g})(B):=\bar{W}\left(\exp \left(\operatorname{Hom}_{F \operatorname{Mod}(R)}\left(\mathfrak{g}^{\vee},(B)\right)\right)\right) \in \mathbb{S}
$$

for $B \in \operatorname{Alg}_{A}(R)$. Here, $\bar{W}$ is the classifying space functor of Definition 1.6, and exp denotes exponentiation of a pro-nilpotent Lie algebra to give a pro-unipotent group.

Observe that this functor is continuous, and denote its left adjoint by $G: F s \operatorname{Aff}(R) \rightarrow$ $F s \hat{\mathcal{N}}(R)$.

Definition 4.20 Define functors

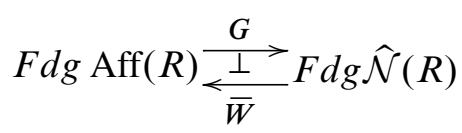

as follows. For $\mathfrak{g} \in F d g \hat{\mathcal{N}}(R)$, the Lie bracket gives a linear map $\bigwedge^{2} \mathfrak{g} \rightarrow \mathfrak{g}$. Write $\Delta$ for the dual $\Delta: \mathfrak{g}^{\vee} \rightarrow \bigwedge^{2} \mathfrak{g}^{\vee}$, which respects the filtration. This is equivalent to a map $\Delta: \mathfrak{g}^{\vee}[-1] \rightarrow \operatorname{Symm}^{2}\left(\mathfrak{g}^{\vee}[-1]\right)$, and we define

$$
O(\bar{W} \mathfrak{g}):=\operatorname{Symm}\left(\mathfrak{g}^{\vee}[-1]\right)
$$


to be the graded polynomial ring on generators $\mathfrak{g}^{\vee}[-1]$, with a derivation defined on generators by $D:=d+\Delta$. The Jacobi identities ensure that $D^{2}=0$.

We define $G$ by writing $\sigma B[1]$ for the brutal truncation (in nonnegative degrees) of $B[1]$, and setting

$$
G(B)^{\vee}=\operatorname{CoLie}(\sigma B[1]),
$$

the free filtered graded Lie coalgebra over $\mathbb{Q}_{\ell}$, with differential similarly defined on cogenerators by $D:=d+\mu, \mu$ here being the product on $B$. Note also that $G(B)$ is cofibrant for all $B$.

Definition 4.21 Define the category $F s \mathcal{P}(R)$ (resp. $F d g \mathcal{P}(R)$ ) to have the fibrant objects of $F s \hat{\mathcal{N}}(R)$ (resp. $F d g \hat{\mathcal{N}}(R)$ ), with morphisms given by

$$
\begin{gathered}
\operatorname{Hom}_{F s \mathcal{P}(R)}(\mathfrak{g}, \mathfrak{h})=\operatorname{Hom}_{\mathrm{Ho}(F s \hat{\mathcal{N}}(R))}(\mathfrak{g}, \mathfrak{h}) \times^{\exp \left(\mathfrak{h}_{0}^{R}\right)} \prod_{x \in \operatorname{Ob} R} \exp \left(\pi_{0} \mathfrak{h}(x)\right), \\
\operatorname{Hom}_{F d g \mathcal{P}(R)}(\mathfrak{g}, \mathfrak{h})=\operatorname{Hom}_{\mathrm{Ho}(F d g \hat{\mathcal{N}}(R))}(\mathfrak{g}, \mathfrak{h}) \times^{\exp \left(\mathfrak{h}_{0}^{R}\right)} \prod_{x \in \mathrm{Ob} R} \exp \left(\mathrm{H}_{0} \mathfrak{h}(x)\right),
\end{gathered}
$$

where $\mathfrak{h}_{0}^{R}$ is the Lie algebra $\operatorname{Hom}_{\operatorname{Mod}(R)}\left(\mathfrak{h}_{0}^{\vee}, \mathbb{Q}_{\ell}\right)=\operatorname{Hom}_{F \operatorname{Mod}(R)}\left(\mathfrak{h}_{0}^{\vee}, \mathbb{Q}_{\ell}\right)$, acting by conjugation on the set of homomorphisms.

Theorem 4.22 There is the following commutative diagram of equivalences of categories:

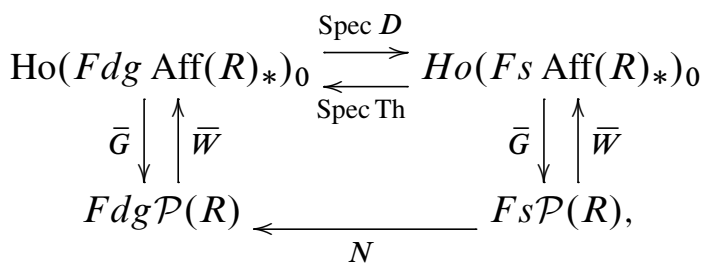

where $N$ denotes normalisation, $D$ is denormalisation, and Th is the functor of ThomSullivan cochains.

Proof The proof of Theorem 2.74 transfers to this context, making use of Lemma 4.14, which implies that everything in the image of $\bar{W}$ is fibrant, as are all objects of $F d g \hat{\mathcal{N}}(R)$ and $F s \hat{\mathcal{N}}(R)$. On objects, the functor $\bar{G}$ is defined by choosing, for any $X \in \operatorname{Ho}\left(F S \operatorname{Aff}(R)_{*}\right)_{0}$ (resp. $\left.X \in \operatorname{Ho}\left(F d g \operatorname{Aff}(R)_{*}\right)_{0}\right)$ a weakly equivalent object $X^{\prime} \in F s \operatorname{Aff}(R)_{00}$ (resp. $\left.X^{\prime} \in F d g \operatorname{Aff}(R)_{00}\right)$, and setting

$$
\bar{G}(X):=G\left(X^{\prime}\right),
$$

for the functor $G$ from Definition 4.20. 
Although we do not have a precise analogue of this result for $\operatorname{Ho}\left(F d g \operatorname{Aff}_{A}(R)\right)$ for general $A$, we do have the following:

Lemma 4.23 Given $X \in \operatorname{Ho}\left(F d g \operatorname{Aff}(R)_{*}\right)_{0}$ and $\mathfrak{g} \in F d g \hat{\mathcal{N}}(R)$,

$$
\begin{aligned}
& \operatorname{Hom}_{\mathrm{Ho}\left(F d g \operatorname{Aff}_{A}(R)_{*}\right)}(X \otimes A, \bar{W} \mathfrak{g} \otimes A) \\
\cong & \operatorname{Hom}_{\mathrm{Ho}\left(F d g \hat{\mathcal{N}}_{A}(R)\right)}(\bar{G}(X) \hat{\otimes} A, \mathfrak{g} \hat{\otimes} A) \times^{\exp \left(\mathfrak{g}_{0}^{R} \hat{\otimes} A\right)} \prod_{x \in \mathrm{Ob} R} \exp \left(\mathrm{H}_{0} \mathfrak{g}(x)\right) .
\end{aligned}
$$

Proof The proof of [37, Proposition 3.48] adapts to this context.

Definition 4.24 We say a filtered cochain algebra $(B, J) \in F D G \operatorname{Alg}_{A}(R)$ is quasiformal if it is weakly equivalent in $F D G \operatorname{Alg}_{A}(R)$ to ${ }_{J} \mathrm{E}_{1}^{*, *}(B)$ (as in Definition 4.11). We say that a filtered homotopy type is quasiformal if its associated cochain algebra is so.

4.3.4 Minimal models Let $F D G \operatorname{Rep}(R)=F D G \operatorname{Mod}_{\mathbb{Q}_{\ell}}(R)$ be the category of nonnegatively graded filtered complexes of $R$-representations.

Definition 4.25 We say that $M \in F D G \operatorname{Rep}(R)$ is minimal if $d\left(J_{m} M\right) \subset J_{m-1} M$ for all $m$.

Lemma 4.26 For any $V \in F D G \operatorname{Rep}(R)$, there exists a quasi-isomorphic filtered subobject $M \hookrightarrow V$, with $M$ minimal.

Proof We prove this by induction on the filtration. Assume that we have constructed a filtered quasi-isomorphism $J_{m} f: J_{m} M \hookrightarrow J_{m} V$ (for $m=-1$, this is trivial). Pick a basis $v_{\alpha}$ for $\mathrm{H}^{*}\left(\mathrm{gr}_{m+1}^{J} V\right)$, and lift $v_{\alpha}$ to $v_{\alpha}^{\prime} \in J_{m+1} V$. Thus $d v_{\alpha}^{\prime} \in J_{m} V$, and $\left[d v_{\alpha}^{\prime}\right]=0 \in \mathrm{H}^{*}\left(J_{m} V / J_{m} M\right)=0$. This means that $d v_{\alpha}^{\prime} \in J_{m} M+d J_{m} V$. Choose $u_{\alpha} \in J_{m} V$ such that $d v_{\alpha}^{\prime}-d u_{\alpha} \in J_{m} M$, and set $\tilde{v}_{\alpha}:=v_{\alpha}^{\prime}-u_{\alpha}$.

Now, $\left[\widetilde{v}_{\alpha}\right]=v_{\alpha} \in \mathrm{H}^{*}\left(\mathrm{gr}_{m+1}^{J} V\right)$, so define

$$
J_{m+1} M:=J_{m} M \oplus\left\langle\widetilde{v}_{\alpha}\right\rangle_{\alpha}
$$

this has the properties that $d J_{m+1} M \subset J_{m} M$ and $\mathrm{H}^{*}\left(\mathrm{gr}_{m+1}^{J} M\right) \cong \mathrm{H}^{*}\left(\operatorname{gr}_{m+1}^{J} V\right)$, as required.

Definition 4.27 We say that a cofibrant object $\mathfrak{m} \in F d g \hat{\mathcal{N}}(R)$ (resp. $F s \hat{\mathcal{N}}(R))$ is minimal if $(\mathfrak{m} /[\mathfrak{m}, \mathfrak{m}])^{\vee}\left(\right.$ resp. $\left.N(\mathfrak{m} /[\mathfrak{m}, \mathfrak{m}])^{\vee}\right)$ is minimal in the sense of Definition 4.25.

Proposition 4.28 (Minimal models) Every weak equivalence class in $F d g \hat{\mathcal{N}}(R)$ (resp. $s \hat{\mathcal{N}}(R)$ ) has a minimal element $\mathfrak{m}$, unique up to nonunique isomorphism.

Proof The proof of [37, Proposition 1.16] adapts to this context, using Lemma 4.26 instead of the corresponding result for $D G \operatorname{Rep}(R)$. 


\subsubsection{Homotopy automorphisms}

Definition 4.29 Given $\mathfrak{u} \in F s \hat{\mathcal{N}}(R)$, let $G=\exp (\mathfrak{u}) \rtimes R$, and define the group presheaf of filtered automorphisms by

$$
\operatorname{Aut}_{J}^{h}(G)(A):=\left\{(f, \theta): f \in \operatorname{Aut}(R)(A), \theta \in \operatorname{Iso}_{F s} \mathcal{P}_{A}(R)\left(\mathfrak{u} \hat{\otimes} A, f^{\sharp} \mathfrak{u} \hat{\otimes} A\right)\right\} .
$$

$\left.\operatorname{Define~RAut~}_{J}(G):=\operatorname{ker} \operatorname{Aut}_{J}^{h}(G) \rightarrow \operatorname{Aut}(R)\right)$.

Definition 4.30 Given $V \in \operatorname{Rep}(R)$ and $\mathfrak{g} \in F s \hat{\mathcal{N}}(R)$, define the spectral sequence ${ }_{J} \mathrm{E}_{*}^{*, *}(R \ltimes \exp (\mathfrak{g}), V)$ to be the cohomology spectral sequence of the filtered complex

$$
O(\bar{W} \mathfrak{g}) \otimes^{R} V,
$$

for $J_{0} V=V$. Thus ${ }_{J} \mathrm{E}_{1}^{a, b}(R \ltimes \exp (\mathfrak{g}), V)=\mathrm{H}^{a+b}\left(\mathrm{Gr}_{-a}^{J} O(\bar{W} \mathfrak{g}) \otimes{ }^{R} V\right)$.

Lemma 4.31 Assume that $G$ is as above, and let $\mathfrak{m} \in F s \hat{\mathcal{N}}(R)$ be a minimal model for $\mathrm{R}_{\mathrm{u}}(G)$. If $\mathrm{H}^{i}(G, V)$ is finite-dimensional for all $i$ and all finite-dimensional irreducible $R$-representations $V$, then the group presheaves

$$
\operatorname{Aut}_{F s \hat{\mathcal{N}}(R)}(\mathfrak{m}) \times \prod_{x \in \mathrm{Ob} R} \exp \left(\pi_{0} \mathfrak{m}(x)\right) \stackrel{\alpha}{\rightarrow} \operatorname{RAut}_{J}(G) \stackrel{\beta}{\rightarrow} \prod_{a, b} \operatorname{Aut}_{R}\left(J_{1} \mathrm{E}_{1}^{a, b}(G, O(R))\right)
$$

are all pro-algebraic groups, the maps $\alpha$ and $\beta$ both have pro-unipotent kernels, and $\beta$ is surjective.

Proof The proof of [37, Theorem 5.13] carries over.

\subsubsection{Examples}

Definition 4.32 Given $B^{\bullet} \in D G \operatorname{Alg}_{A}(R)$, we define the good truncation $\tau_{*}$ on $B$ by

$$
\left(\tau_{m} B\right)^{n}:= \begin{cases}B^{n} & n<m \\ \mathrm{Z}^{m}(B) & n=m \\ 0 & n>m\end{cases}
$$

Observe that $\left(B^{\bullet}, \tau\right) \in F D G \operatorname{Alg}_{A}(R)$.

Definition 4.33 Given a bicosimplicial algebra $B^{\bullet, \bullet} \in c c \operatorname{Alg}_{A}(R)$, we define the associated filtered cosimplicial algebra $\left(\tau_{0}^{\prime \prime} B \leq \tau_{1}^{\prime \prime} B \leq \cdots\right) \in F c \operatorname{Alg}_{A}(R)$ by

$$
\left(\tau_{m}^{\prime \prime} B\right)^{n}=\left(D \tau_{m} \operatorname{Th} B^{n, \bullet}\right)^{n},
$$

for $D$, Th as in Proposition 2.70. Observe that there is a canonical quasi-isomorphism $\operatorname{diag} B^{\bullet, \bullet} \rightarrow \tau_{\infty}^{\prime \prime} B^{\bullet}$, where diag denotes the diagonal of a bicosimplicial complex. 
In practice, the only filtered homotopy types which we will encounter come from morphisms of spaces:

Definition 4.34 Given an algebraic variety $X$ and an ind-constructible $\ell$-adic sheaf $\mathbb{V}$ on $X$, recall (eg from [36, Definition 2.3]) that there is a natural cosimplicial complex

$$
\mathscr{C}_{\text {et }}^{\bullet}(\mathbb{V})
$$

of $\ell$-adic sheaves on $\mathbb{V}$, with the property that $\Gamma\left(X, \mathscr{C}_{\text {et }}^{\bullet}(\mathbb{V})\right)=\mathrm{C}_{\text {et }}^{\bullet}(X, \mathbb{V})$, the Godement resolution (as in Example 3.18). This construction respects tensor products.

Lemma 4.35 To any morphism $j: Y \rightarrow X$ of algebraic varieties, and any $\mathbb{Q}_{\ell}$-sheaf $\mathscr{S}$ of algebras on $Y$ as in Definition 4.34, there is associated a canonical filtered homotopy type $\mathrm{C}_{\text {et }}^{\bullet}(j, \mathscr{Y}) \in \operatorname{Ho}\left(F c \operatorname{Alg}_{\mathbb{Q}_{\ell}}\right)$, with the property that ${ }_{J} \mathrm{E}_{*}^{*, *} \mathrm{C}_{\text {et }}^{\bullet}(j, \mathscr{S})$ is the Leray spectral sequence

$$
{ }_{J} \mathrm{E}_{1}^{a, b} \mathrm{C}_{\mathrm{et}}^{\bullet}(j, \mathscr{Y})=\mathrm{H}^{2 a+b}\left(X, \mathbf{R}^{-a} j_{*} \mathscr{Y}\right) \Longrightarrow \mathrm{H}^{a+b}(Y, \mathscr{Y}) .
$$

The associated unfiltered homotopy type is canonically weakly equivalent to $\mathrm{C}_{\mathrm{et}}^{\bullet}(Y, \mathscr{S})$.

Proof We have a $\mathbb{Q}_{\ell}$-sheaf $j_{*} \mathscr{C}_{\text {ét }}^{\bullet}(\mathscr{S})$ of cosimplicial algebras on $X$, and hence a bicosimplicial algebra

$$
\mathrm{C}_{\mathrm{et}}^{\bullet}\left(X, j_{*} \mathscr{b}_{\mathrm{et}}^{\bullet}(\mathscr{S})\right) \text {. }
$$

Now, set

$$
J_{n} \mathrm{C}_{\text {et }}^{\bullet}(j, \mathscr{S})=\tau_{n}^{\prime \prime} \mathrm{C}_{\text {et }}^{\bullet}\left(X, j_{*} \mathscr{C}_{\text {et }}^{\bullet}(\mathscr{S})\right)=\operatorname{diag} \mathrm{C}_{\text {et }}^{\bullet}\left(X, D \tau_{n} \operatorname{Th} j_{*} \mathscr{C}_{\text {et }}^{\bullet}(\mathscr{S})\right),
$$

as in Definition 4.33, with $\mathrm{C}_{\text {ét }}^{\bullet}\left(X, j_{*} \mathscr{C}_{\text {êt }}^{\bullet}(\mathscr{S})\right) \rightarrow J_{\infty} \mathrm{C}_{\text {êt }}^{\bullet}(j, \mathscr{S})$ a quasi-isomorphism.

Finally, observe that there is a quasi-isomorphism

$$
\mathrm{C}_{\text {ét }}^{\bullet}(Y, \mathscr{S})=\Gamma\left(X, j_{*} \mathscr{C}_{\text {ét }}^{\bullet}(\mathscr{S})\right) \rightarrow \operatorname{diag} \mathrm{C}_{\text {ét }}^{\bullet}\left(X, j_{*} \mathscr{G}_{\text {èt }}^{\bullet}(\mathscr{Y})\right),
$$

and that $\operatorname{gr}_{n}^{\tau} j_{*} \mathscr{C}_{\mathrm{et}}^{\bullet}(\mathscr{Y})$ is quasi-isomorphic to $\mathbf{R}^{n} j_{*} \mathscr{Y}$.

Remark 4.36 There is a similar statement for filtrations on homotopy types coming from morphisms of topological spaces, using Čech resolutions instead of Godement resolutions.

Since the construction above is functorial, for any point $y \in Y$, we have a morphism $\mathrm{C}_{\text {ét }}^{\bullet}(j, \mathscr{S}) \rightarrow \mathrm{C}_{\text {et }}^{\bullet}\left(\operatorname{id}_{y}, \mathscr{S}_{y}\right)$, where $\mathrm{id}_{y}$ is the identity map $\operatorname{id}_{y}: y \rightarrow y$. Now,

$$
J_{n} \mathrm{C}_{\text {ét }}^{\bullet}\left(\mathrm{id}_{y}, \mathscr{S}_{y}\right)=\operatorname{diag} \mathrm{C}_{\text {ét }}^{\bullet}\left(y, D \tau_{n} \operatorname{Th} \mathscr{S}_{y}\right) .
$$

Since $\mathscr{S}_{y}$ has constant simplicial structure, Th $\mathscr{S}_{y}=\mathscr{Y}_{y}$, so $J_{n} \mathrm{C}_{\mathrm{et}}^{\bullet}\left(\operatorname{id}_{y}, \mathscr{S}_{y}\right)=\mathscr{Y}_{y}$ for all $n \geq 0$. 
Definition 4.37 Given a morphism $j: Y \rightarrow X$ of algebraic varieties and a Zariskidense continuous map

$$
\rho: \overline{\pi_{f}^{\text {ét }}(Y)} \rightarrow R\left(\mathbb{Q}_{\ell}\right)
$$

we define the filtered homotopy type $\left(Y^{\rho, \mathrm{Mal}}, j\right)$ to correspond to $\mathrm{C}_{\text {ét }}^{\bullet}(j, \mathbb{O}(R)) \in$ $F c \operatorname{Alg}(R)_{*}$, where the augmentation map is the canonical morphism

$$
\mathrm{C}_{\mathrm{èt}}^{\bullet}(j, \mathbb{O}(R)) \rightarrow \prod_{y \in Y} \mathrm{C}_{\mathrm{èt}}^{\bullet}\left(\mathrm{id}_{y}, \mathbb{O}(R)\right)=\prod_{y \in Y} O(R)(y,-) .
$$

\section{Algebraic Galois actions}

\subsection{Weight decompositions}

By a weight decomposition, we will mean an algebraic action of the group $\mathbb{G}_{m}$. A weight decomposition on a vector space $V$ is equivalent to a decomposition $V=$ $\bigoplus_{n \in \mathbb{Z}} \mathcal{W}_{n} V$, given by $\lambda \in \mathbb{G}_{m}$ acting as $\lambda^{n}$ on $\mathcal{W}_{n} V$.

Fix a prime $p$, which need not differ from $\ell$. Let $\mathbb{Z}^{\text {alg }}$ be the pro-algebraic group over $\mathbb{Q}_{\ell}$ parametrising $\mathbb{Z}$-representations. Since $\mathbb{Z}$ is commutative, $\mathbb{Z}^{\text {alg }}$ is commutative, so $\mathbb{Z}^{\text {alg }}=\mathbb{Z}^{\text {red }} \times R_{\mathrm{u}}\left(\mathbb{Z}^{\text {alg }}\right)$, where $\mathbb{Z}^{\text {red }}$ is its reductive quotient. For any unipotent algebraic group $U$, this means that $\operatorname{Hom}\left(\mathrm{R}_{\mathrm{u}}\left(\mathbb{Z}^{\text {alg }}\right), U\right) \cong \operatorname{Hom}\left(\mathbb{Z}, U\left(\mathbb{Q}_{\ell}\right)\right)=U\left(\mathbb{Q}_{\ell}\right)$, so $\mathrm{R}_{\mathrm{u}}\left(\mathbb{Z}^{\text {alg }}\right)=\mathbb{G}_{a}$. Combining these observations gives $\mathbb{Z}^{\text {alg }}=\mathbb{G}_{a} \times \mathbb{Z}^{\text {red }}$.

Likewise, let $\widehat{\mathbb{Z}}^{\text {alg }}$ be the pro-algebraic group over $\mathbb{Q}_{\ell}$ parametrising continuous $\widehat{\mathbb{Z}}-$ representations. Since continuous $\widehat{\mathbb{Z}}$-representations form a full subcategory of $\mathbb{Z}-$ representations, $\widehat{\mathbb{Z}}^{\text {alg }}$ is a quotient of $\mathbb{Z}^{\text {alg }}$. The reasoning above adapts to show that $\widehat{\mathbb{Z}}^{\text {alg }}=\mathbb{G}_{a} \times \widehat{\mathbb{Z}}^{\text {red }}$.

Definition 5.1 Given $n \in \mathbb{Z}$ and a power $q$ of $p$, recall that an element $\alpha \in \overline{\mathbb{Q}}$ is said to be pure of weight $n$ if it is algebraic and for every embedding $\iota: \overline{\mathbb{Q}} \hookrightarrow \hookrightarrow \mathbb{C}$ the element $\iota(\alpha)$ has complex absolute value $q^{n / 2}$.

Let $M_{q}$ be the quotient of $\hat{\mathbb{Z}}^{\text {red }}$ whose representations $\rho$ correspond to semisimple $\widehat{\mathbb{Z}}$-representations for which the eigenvalues of $\rho(1)$ are all of integer weight with respect to $q$. Such representations are called mixed.

Observe that every $M_{q}$-representation decomposes into "pure" representations, in which all eigenvalues have the same weight. There is thus a canonical map $\mathbb{G}_{m} \rightarrow M_{q}$ given by $\lambda \in \mathbb{G}_{m}$ acting as $\lambda^{n}$ on a pure representation of weight $n$. 
Definition 5.2 Define $P_{q}$ to be the quotient of $M_{q}$ whose representations are pure of weight 0 , so $P_{q}=M_{q} / \mathbb{G}_{m}$.

Definition 5.3 Given $n \in \mathbb{Z}$, an embedding $\iota: \overline{\mathbb{Q}_{\ell}} \rightarrow \mathbb{C}$ and a power $q$ of $p$, recall that an element $\alpha \in \overline{\mathbb{Q}_{\ell}}$ is said to be $\iota$-pure of weight $n$ if $|\iota(\alpha)|=q^{n / 2}$.

Let $M_{\iota, q}$ be the quotient of $\mathbb{Z}^{\text {red }}$ whose representations $\rho$ correspond to semisimple $\mathbb{Z}$-representations for which the eigenvalues of $\rho(1)$ are all of integer $\iota$-weight. Note that $M_{q}$ is a quotient of $M_{\iota, q}$.

Observe that there is a canonical map $\mathbb{G}_{m} \rightarrow M_{\iota, q}$ given by $\lambda \in \mathbb{G}_{m}$ acting as $\lambda^{n}$ on an $\iota$-pure representation of weight $n$, and that this induces the map $\mathbb{G}_{m} \rightarrow M_{q}$ above.

Definition 5.4 Define $P_{\iota, q}$ to be the quotient of $M_{\iota, q}$ whose representations are pure of $\iota$-weight 0 , so $P_{\iota, q}=M_{\iota, q} / \mathbb{G}_{m}$.

Definition 5.5 Given a pro-algebraic group $G$, let $G^{0}$ be the connected component of the identity; if $\widehat{G}$ is the maximal pro-finite quotient of $G$ (parametrising representations with finite monodromy), then $G^{0}=\operatorname{ker}(G \rightarrow \widehat{G})$.

Lemma 5.6 If $\Gamma$ is a pro-discrete group, then we may make the identification

$$
\Gamma^{\mathrm{alg}, 0}=\underset{\Delta}{\lim } \Delta^{\mathrm{alg}}
$$

where $\Delta$ runs over $\Delta \triangleleft \Gamma$ open of finite index.

Therefore the category of finite-dimensional $\Gamma^{\mathrm{alg}, 0}$-representations is the direct limit $\lim _{\longrightarrow \Delta} \operatorname{FD} \operatorname{Rep}(\Delta)$ (over $\Delta$ as above) of the categories of finite-dimensional $\Delta$-representations.

Proof This is essentially [29, Proposition 2], which deals with the case when $\Gamma$ is discrete, and refers to $\lim _{\rightarrow} \operatorname{FD} \operatorname{Rep}(\Delta)$ as the category of virtual $\Gamma$-representations.

First note that $\widehat{\Gamma^{\mathrm{alg}}}=\widehat{\Gamma}$, where the pro-finite completion $\widehat{\Gamma}$ of $\Gamma$ is characterised by the property that $\operatorname{Hom}_{\text {pro(Gp) }}(\Gamma, F) \cong \operatorname{Hom}_{\text {pro(Gp) }}(\widehat{\Gamma}, F)$ for all finite groups $F$. Thus $\widehat{\Gamma}=\Gamma$ whenever $\Gamma$ is pro-finite.

The exact sequence $\Delta \rightarrow \Gamma \rightarrow \Gamma / \Delta \rightarrow 1$ gives an exact sequence $(\Delta)^{\text {alg }} \stackrel{\alpha}{\rightarrow} \Gamma^{\text {alg }} \rightarrow$ $\Gamma / \Delta \rightarrow 1$. It suffices to show that $\alpha$ is injective. This follows from the observation that every finite-dimensional $\Delta$-representation $V$ embeds into a finite-dimensional $\Gamma$-representation $\operatorname{Ind}_{\Delta}^{\Gamma} V$. 
Thus if $F$ is a generator for $\mathbb{Z}$, then representations of $\mathbb{Z}^{\text {alg, } 0}$ are sums of $F^{r}$ representations, with morphisms commuting locally with sufficiently high powers of $F$.

Observe that we have commutative diagrams

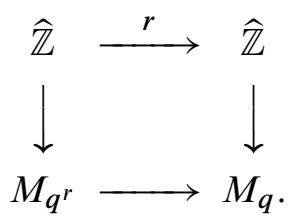

Any $\widehat{\mathbb{Z}}$-representation with finite monodromy is pure of weight 0 , giving a map $P_{p} \rightarrow \widehat{\mathbb{Z}}$. Also note that $M_{q^{r}}=\operatorname{ker}\left(M_{q} \rightarrow \mathbb{Z} / r \mathbb{Z}\right)$. Combining these observations gives:

Lemma 5.7 We have

$$
M_{p}^{0}=\lim _{\longleftarrow} M_{p^{r}}, \quad P_{p}^{0}=\lim _{\leftarrow} P_{p^{r}} .
$$

Writing $M^{0}:=M_{p}^{0}$ and $P^{0}:=P_{p}^{0}$, there are quotient maps $\widehat{\mathbb{Z}}^{\text {red,0 }} \rightarrow M^{0} \rightarrow P^{0}$. There are similar results for $M_{\iota}^{0}:=M_{\iota, p}^{0}, P_{\iota}^{0}:=P_{\iota, p}^{0}$.

Definition 5.8 We say that a representation of $\mathbb{Z}^{\text {alg,0 } 0}$ is mixed (resp. pure of weight 0 , resp. ८-mixed with integral weights, resp. ८-pure) if the action of $\mathbb{Z}^{\text {red, } 0} \triangleleft \mathbb{Z}^{\text {alg, } 0}$ factors through $M^{0}$ (resp. $P^{0}$, resp. $M_{\iota}^{0}$, resp. $\left.P_{\iota}^{0}\right)$.

Lemma 5.9 Observe that the canonical maps $\mathbb{G}_{m} \rightarrow M_{q}$ are compatible, giving $\mathbb{G}_{m} \rightarrow M^{0}$, with trivial image in $P^{0}$. Similarly, we have $\mathbb{G}_{m} \rightarrow M_{\iota}^{0}$, with trivial image in $P_{\iota}^{0}$.

\subsubsection{Slope decompositions}

Definition 5.10 Define the pro-algebraic group $\widetilde{\mathbb{G}_{m}}$ to be the inverse limit of the étale universal covering system of $\mathbb{G}_{m}$. This is the inverse system $\left\{G_{r}\right\}_{r \in \mathbb{N}}$ with $G_{r}=\mathbb{G}_{m}$ and morphisms $[s]: G_{s r} \rightarrow G_{r}$, for $s \in \mathbb{N}$.

Lemma 5.11 The category of $\widetilde{\mathbb{G}_{m}}$-representations is canonically equivalent to the category of $\mathbb{Q}$-graded vector spaces. 
Proof A representation of $\mathbb{G}_{m}$ is equivalent to a $\mathbb{Z}$-grading. Given a finite-dimensional vector space $V$ with a $\mathbb{Q}$-grading $V=\bigoplus V_{\lambda}$, let $d$ be the lowest common multiple of the denominators of the set $\left\{\lambda \in \mathbb{Q}: V_{\lambda} \neq 0\right\}$. Then $V=\bigoplus_{n \in \mathbb{Z}} V_{n / d}$, giving a $\mathbb{G}_{m}$-action on $V$. If we regard this copy of $\mathbb{G}_{m}$ as $G_{d}$, this defines a $\mathbb{G}_{m}$-action.

Now, for any pro-algebraic group $G$, arbitrary $G$-representations are nested unions of finite-dimensional $G$-subrepresentations. Likewise, every $\mathbb{Q}$-graded vector space can be expressed as a nested union of finite-dimensional $\mathbb{Q}$-graded vector subspaces, so the two categories are equivalent.

Now assume that $p=\ell$.

Definition 5.12 Given a power $q$ of $p$, normalise the $p$-adic valuation $v$ on $\overline{\mathbb{Q}}_{p}$ by $v(q)=1$. Define the slope of $\alpha \in \overline{\mathbb{Q}}_{p}$ to be $v(\alpha) \in \mathbb{Q}$.

Lemma 5.13 There is a canonical morphism $\widetilde{\mathbb{G}_{m}} \rightarrow \mathbb{Z}^{\text {red }}$, corresponding to the functor sending a $\mathbb{Z}$-representation $V$ to a slope decomposition $\bigoplus V_{\lambda}$.

Proof Let $F$ be the canonical generator for $\mathbb{Z}$. Given a finite-dimensional semisimple $\mathbb{Z}$-representation $V$, we may decompose $V \otimes_{\mathbb{Q}_{p}} \overline{\mathbb{Q}}_{p}$ into $F$-eigenspaces, and hence take a decomposition by slopes of the eigenvalues. Since conjugates in $\overline{\mathbb{Q}}_{p}$ have the same slope, this descends to a slope decomposition $V=\bigoplus_{\lambda \in \mathbb{Q}} V_{\lambda}$, as required.

\subsection{Potentially unramified actions}

Fix a prime $p \neq \ell$, and take a local field $K$, with finite residue field $k$ of characteristic $p$. Let $\mathcal{G}:=\operatorname{Gal}(\bar{K} / K)^{\text {alg }}$, the pro-algebraic completion of $\operatorname{Gal}(\bar{K} / K)$ over $\mathbb{Q}_{\ell}$.

Definition 5.14 A finite-dimensional continuous $\mathbb{Q}_{\ell}$-representation of $\operatorname{Gal}(\bar{K} / K)$ is potentially unramified if there exists a finite extension $K^{\prime} / K$ for which the action of $\operatorname{Gal}\left(\bar{K} / K^{\prime}\right)$ is unramified. Say that an arbitrary $\mathbb{Q}_{\ell}$-representation of $\operatorname{Gal}(\bar{K} / K)$ is potentially unramified if it is a sum of finite-dimensional potentially unramified representations.

These form a neutral Tannakian category (see Definition 2.7); let $\mathcal{G}^{\text {pnr }}$ be the corresponding pro-algebraic group. Since $\operatorname{Rep}\left(\mathcal{G}^{\mathrm{pnr}}\right)$ is a Tannakian subcategory of $\operatorname{Rep}(\mathcal{G})$, $\mathcal{G}^{\text {pnr }}$ is a quotient of $\mathcal{G}$.

Lemma 5.15 We can write $\mathcal{G}^{\mathrm{pnr}}=\operatorname{Gal}(\bar{k} / k)^{\mathrm{alg}} \times_{\mathrm{Gal}(\bar{k} / k)} \mathrm{Gal}(\bar{K} / K)$, so $\mathcal{G}^{\mathrm{pnr}, 0}=$ $\operatorname{Gal}(\bar{k} / k)^{\mathrm{alg}, 0} \cong \widehat{\mathbb{Z}}^{\mathrm{alg}, 0}$. 
Proof A representation $\mathcal{G} \rightarrow \mathrm{GL}(V)$ (for $V$ finite-dimensional) is potentially unramified if it annihilates $\operatorname{ker}\left(\operatorname{Gal}\left(\bar{K} / K^{\prime}\right) \rightarrow \operatorname{Gal}\left(\bar{k} / k^{\prime}\right)\right)$ for some finite Galois extension $K^{\prime} / K$. In other words, it annihilates $\operatorname{ker}\left(\operatorname{Gal}(\bar{K} / K) \rightarrow \operatorname{Gal}(\bar{k} / k) \times_{\operatorname{Gal}\left(k^{\prime} / k\right)}\right.$ $\left.\operatorname{Gal}\left(K^{\prime} / K\right)\right)$, so is an algebraic representation of $\operatorname{Gal}(\bar{k} / k)^{\text {alg }} \times_{\operatorname{Gal}\left(k^{\prime} / k\right)} \operatorname{Gal}\left(K^{\prime} / K\right)$. Thus the category of finite-dimensional $\mathcal{G}^{\mathrm{pnr}}$-representations is given by

$$
\begin{aligned}
\operatorname{FD} \operatorname{Rep}\left(\mathcal{G}^{\mathrm{pnr}}\right) & =\underset{K^{\prime}}{\lim } \operatorname{FD} \operatorname{Rep}\left(\operatorname{Gal}(\bar{k} / k)^{\operatorname{alg}} \times_{\operatorname{Gal}\left(k^{\prime} / k\right)} \operatorname{Gal}\left(K^{\prime} / K\right)\right) \\
& =\operatorname{FD} \operatorname{Rep}\left({\overleftarrow{K^{\prime}}}_{\lim } \operatorname{Gal}(\bar{k} / k)^{\operatorname{alg}} \times_{\operatorname{Gal}\left(k^{\prime} / k\right)} \operatorname{Gal}\left(K^{\prime} / K\right)\right) \\
& =\operatorname{FD} \operatorname{Rep}\left(\operatorname{Gal}(\bar{k} / k)^{\mathrm{alg}} \times_{\operatorname{Gal}(\bar{k} / k)} \operatorname{Gal}(\bar{K} / K)\right),
\end{aligned}
$$

as required.

The final statement is an immediate consequence of Lemma 5.6.

Definition 5.16 We say that a representation of $\mathcal{G}^{\mathrm{pnr}}$ is mixed (resp. pure of weight 0 ) if the resulting action of $\mathbb{Z}^{\mathrm{alg}, 0} \rightarrow \widehat{\mathbb{Z}}^{\mathrm{alg}, 0}$ is so.

\subsection{Potentially crystalline actions}

Now let $\ell=p$, and take a local field $K$, with finite residue field $k$ of order $q=p^{f}$. Let $\mathcal{G}:=\operatorname{Gal}(\bar{K} / K)^{\text {alg }}$, the pro-algebraic completion of $\operatorname{Gal}(\bar{K} / K)$ over $\mathbb{Q}_{p}$. Let $W:=W(k)$, with fraction field $K_{0}$, and let $\sigma$ denote the unique lift of arithmetic Frobenius $\Phi \in \operatorname{Gal}\left(\bar{k} / \mathbb{F}_{p}\right)$ to $\sigma \in \operatorname{Gal}\left(K_{0}^{\mathrm{nr}} / \mathbb{Q}_{p}\right)$, for $K_{0}^{\mathrm{nr}}$ the maximal unramified extension of $K_{0}$. Note that the geometric Frobenius of the previous section is $F=\Phi^{-f}$.

Definition 5.17 Say that a finite-dimensional continuous $\operatorname{Gal}(\bar{K} / K)$-representation over $\mathbb{Q}_{p}$ is potentially crystalline if there exists a finite extension $K^{\prime} / K$ for which the action of $\operatorname{Gal}\left(\bar{K} / K^{\prime}\right)$ is crystalline. Say that an arbitrary $\mathbb{Q}_{p}$-representation of $\operatorname{Gal}(\bar{K} / K)$ is potentially crystalline if it is a sum of finite-dimensional potentially crystalline representations. Note that since unramified representations are automatically crystalline, all potentially unramified representations are potentially crystalline.

These form a neutral Tannakian category (see Definition 2.7); let $\mathcal{G}^{\text {pcris }}$ be the corresponding pro-algebraic group. $\operatorname{Since} \operatorname{Rep}\left(\mathcal{G}^{\text {pcris }}\right)$ is a full subcategory of $\operatorname{Rep}(\mathcal{G})$ closed under subobjects, $\mathcal{G}^{\text {pcris }}$ is a quotient of $\mathcal{G}$.

Definition 5.18 In $\left[9\right.$, Section 4], Fontaine defined a ring $B_{\text {cris }}:=B_{\text {cris }}(V)$ of periods over $\mathbb{Q}_{p}$, equipped with a Hodge filtration and actions of $\operatorname{Gal}(\bar{K} / K)$ and Frobenius, 
and used it to characterise crystalline representations (adapted in Proposition 5.20 below).

In [33, 6.8], Olsson defined a localisation $\widetilde{B}_{\text {cris }}(V)$ of $B_{\text {cris }}(V)$ as follows. Fix a sequence $\tau_{m}$ of elements of $\bar{V}$ with $\tau_{0}=p$ and $\tau_{m+1}^{p}=\tau_{m}$ for all $m \geq 0$. Define $\lambda_{p^{-n}}$ to be the sequence $\left(\tau_{n+m}\right)_{m \geq 0}$, and let $\delta_{p^{-n}}$ be the associated Teichmüller lifting. Set

$$
\widetilde{B}_{\text {cris }}(V):=B_{\text {cris }}(V)\left[\delta_{p^{-n}}^{-1}\right]_{n \geq 0},
$$

noting that $\left(\delta_{p^{-n-1}}\right)^{p}=\delta_{p^{-n}}$.

Definition 5.19 Given a finite-dimensional $\operatorname{Gal}(\bar{K} / K)$-representation $U$, set

$$
\begin{aligned}
D_{\text {cris }, \mathrm{K}}(U) & :=\left(U \otimes_{\mathbb{Q}_{p}} B_{\text {cris }}\right)^{\mathrm{Gal}(\bar{K} / K),} \\
D_{\text {pcris }}(U) & :=\lim _{\text {cris, } \mathrm{K}^{\prime}}(U), \\
\widetilde{D}_{\text {cris }, \mathrm{K}}(U) & :=\left(U \otimes_{\mathbb{Q}_{p}} \widetilde{B}_{\text {cris }}\right)^{\mathrm{Gal}(\bar{K} / K),} \\
\widetilde{D}_{\text {pcris }}(U) & :=\lim _{\longrightarrow} \widetilde{D}_{\text {cris, } \mathrm{K}^{\prime}}(U),
\end{aligned}
$$

for $K^{\prime}$ ranging over all finite extensions of $K$. For an arbitrary algebraic $\operatorname{Gal}(\bar{K} / K)-$ representation $U$, set

$$
D_{\text {pcris }}(U):=\underline{\lim } D_{\text {pcris }}\left(U_{\alpha}\right),
$$

for $U_{\alpha}$ running over all finite-dimensional subrepresentations, and similarly for $\widetilde{D}_{\text {pcris }}$.

Observe that Spec $B_{\text {cris }}$ is an affine $\mathcal{G}$-scheme over $\operatorname{Spec} \mathbb{Q}_{p}$, and that the coarse quotient $\left(\operatorname{Spec} B_{\text {cris }}\right) / \mathcal{G}^{0}$ is $\operatorname{Spec} K_{0}^{\mathrm{nr}}$.

Proposition 5.20 An action of $\mathcal{G}$ on an affine $\mathbb{Q}_{p}-$ scheme $Y$ factors through $\mathcal{G}^{\text {pcris }}$ if and only if there exists an affine $K_{0}^{\mathrm{nr}}$-scheme $Z$, with

$$
Y \times \mathbb{Q}_{p} \text { Spec } \widetilde{B}_{\text {cris }} \cong Z \times_{K_{0}^{\text {nr }}} \operatorname{Spec} \widetilde{B}_{\text {cris }}
$$

a $\mathcal{G}^{0}$-equivariant map (for trivial $\mathcal{G}^{0}$-action on $Z$ ).

In that case, we necessarily have $\mathbb{O}_{Z}=D_{\text {pcris }}\left(\mathscr{O}_{Y}\right)=\widetilde{D}_{\text {pcris }}\left(\mathscr{O}_{Y}\right)$.

Proof If we replace potentially crystalline with crystalline, and $K_{0}^{\text {nr }}$ with $K_{0}$, then this is just [33, Theorem D.3]. Taking the direct limit over finite extensions of $K$ gives the first expression.

Taking $\mathcal{G}^{0}$-invariants gives $\mathbb{O}_{Z}=\widetilde{D}_{\text {pcris }}\left(\mathscr{O}_{Y}\right)$, but then [33, Remark D.10] shows that for potentially crystalline representations $U, \widetilde{D}_{\text {pcris }}(U)=D_{\text {pcris }}(U)$. 
5.3.1 Frobenius actions Although we do not have a canonical map $\mathbb{Z}^{\text {alg, } 0} \rightarrow \mathcal{G}^{\text {pcris }}$, there is something nearly as strong:

\section{Lemma 5.21 There is a canonical morphism}

$$
\mathbb{Z}^{\mathrm{alg}, 0} \otimes_{\mathbb{Q}_{p}} B_{\text {cris }}^{\sigma} \rightarrow \mathcal{G}^{\text {pcris }} \otimes_{\mathbb{Q}_{p}} B_{\text {cris }}^{\sigma}
$$

of affine group schemes over the $\sigma$-invariant subring $B_{\text {cris }}^{\sigma}$ of $B_{\text {cris }}$.

Proof Given $U \in \operatorname{FD} \operatorname{Rep}\left(\mathcal{G}^{\text {pcris }}\right), U$ is crystalline over $K^{\prime}$ for some finite extension $K^{\prime} / K$ with residue field $k^{\prime}$. If $\left|k^{\prime} / k\right|=r$ and $q=p^{f}$, then $\phi^{f r}$ is a $K_{0}^{\prime}$-linear endomorphism of $D_{\text {cris, } \mathrm{K}^{\prime}}(U)$. This extends uniquely to give a $K_{0}^{\mathrm{nr}}$-linear automorphism $F_{r}$ of $D_{\text {pcris }}(U)$ (note that $F_{r} \neq \phi^{f r}$, the latter being $\sigma$-semilinear).

Now, observe that $D_{\text {pcris }}(U)$ is a sum of finite-dimensional $F_{r}$-representations over $\mathbb{Q}_{p}$, since $D_{\text {cris, } K^{\prime}}(U)$ is finite-dimensional over $K^{\prime}$, and hence over $\mathbb{Q}_{p}$. This gives us a $\sigma$-equivariant $\mathbb{Q}_{p}$-linear action of $\mathbb{Z}^{0 \text {,alg }}$ on $D_{\text {pcris }}(U)$, and hence a $\sigma$-equivariant $B_{\text {cris }}^{\sigma}$-linear action on $D_{\text {pcris }}(U) \otimes_{K_{0}^{\text {nr }}} B_{\text {cris }}=U \otimes_{\mathbb{Q}_{p}} B_{\text {cris }}$. We now take the $\phi$-invariant subspace, giving a $\mathbb{Z}^{0, \text { alg }} \otimes_{\mathbb{Q}_{p}} B_{\text {cris }}^{\sigma}$-action on $U \otimes_{\mathbb{Q}_{p}} B_{\text {cris }}^{\sigma}$.

If we took a larger extension $K^{\prime \prime} / K$ with residue field $k^{\prime \prime}$, then we would have $\left|k^{\prime \prime} / k\right|=s$ with $r \mid s$. The corresponding $K_{0}^{\mathrm{nr}}$-linear automorphism $F_{s}$ of $D_{\text {pcris }}(U)$ is given by $F_{s}=F_{r}^{s / r}$, so gives rise to the same $\mathbb{Z}^{0, \text { alg }}$-action on $D_{\text {pcris }}(U)$. This ensures that the action is functorial in $U$.

Given $U, V \in \operatorname{FD} \operatorname{Rep}\left(\mathcal{G}^{\text {pcris }}\right)$, we have $D_{\text {pcris }}\left(U \otimes \mathbb{Q}_{p} V\right)=D_{\text {pcris }}(U) \otimes_{K_{0}^{\text {nr }}} D_{\text {pcris }}(V)$, compatible with $\phi$. Choosing $K^{\prime}$ so that $U, V$ are both crystalline over $K^{\prime}$, we see that $D_{\text {pcris }}\left(U \otimes_{\mathbb{Q}_{p}} V\right)$ is isomorphic to $D_{\text {pcris }}(U) \otimes_{K_{0}^{\text {nr }}} D_{\text {pcris }}(V)$ as an $F_{r}$-representation. Hence the $\mathbb{Z}^{0 \text {,alg }} \otimes_{\mathbb{Q}_{p}} B_{\text {cris }}^{\sigma}$-representation $\left(U \otimes_{\mathbb{Q}_{p}} V\right) \otimes_{\mathbb{Q}_{p}} B_{\text {cris }}^{\sigma}$ is isomorphic to $\left(U \otimes_{\mathbb{Q}_{p}} B_{\text {cris }}^{\sigma}\right) \otimes_{B_{\text {cris }}^{\sigma}}\left(V \otimes_{\mathbb{Q}_{p}} B_{\text {cris }}^{\sigma}\right)$.

For a $\mathbb{Q}_{p}$-algebra $A$, Tannakian duality says that giving an element $g \in \mathcal{G}^{\text {pcris }}(A)$ is equivalent to giving $A$-linear automorphisms $g_{U}$ of $U \otimes A$ for all $\mathcal{G}^{\text {pcris }}$-representations $U$, functorial and compatible with tensor products and duals. Therefore the $\mathbb{Z}^{0, \text { alg }} \otimes_{\mathbb{Q}_{p}} B_{\text {cris }}^{\sigma}$-actions on the representations $U \otimes_{\mathbb{Q}_{p}} B_{\text {cris }}^{\sigma}$ give group homomorphisms $\mathbb{Z}^{0, \text { alg }}(C) \rightarrow \mathcal{G}^{\text {pcris }}(C)$, functorial in $B_{\text {cris }}^{\sigma}$-algebras $C$, as required.

Definition 5.22 We say that a potentially crystalline representation $U$ is mixed (resp. pure, resp. เ-mixed with integral weights, resp. ८-pure) if the action of $\mathbb{Z}^{\mathrm{alg}, 0} \otimes B_{\text {cris }}^{\sigma}$ on $U \otimes B_{\text {cris }}^{\sigma}$ factors through $M_{q}$ (resp. $P_{q}$, resp. $M_{\iota, q}$, resp. $P_{\iota, q}$ ). This is equivalent to saying that the action of $\mathbb{Z}$ on $D_{\text {pcris }}(U)$ is mixed (resp. pure, resp. $\iota-$ mixed with integral weights, resp. ८-pure). 
We have the following analogue of a slope decomposition:

Lemma 5.23 There is a canonical morphism $\widetilde{\mathbb{G}_{m}} \rightarrow \mathcal{G}^{\text {pcris }} \otimes_{\mathbb{Q}_{p}} B_{\text {cris }}^{\sigma}$ of affine group schemes over $B_{\text {cris }}^{\sigma}$, for $\widetilde{\mathbb{G}_{m}}$ as in Definition 5.10.

Proof Combine Lemma 5.13 with Lemma 5.21.

\section{Varieties over finite fields}

Fix a variety $X_{k}$ over a finite field $k$, of order $q$ prime to $\ell$. Let $X:=X_{k} \otimes_{k} \bar{k}$, for $\bar{k}$ the algebraic closure of $k$. There is a Galois action on $X$, and hence on the pro-simplicial set $X_{\text {ét }}$, and on its algebraisation $G\left(X_{\text {et }}\right)^{\text {alg }}$. The purpose of this section is to describe this action as far as possible.

\subsection{Algebraising the Weil groupoid}

The morphism $X \rightarrow X_{k}$ gives a map of groupoids $\alpha: \pi_{f}^{\text {ét }} X \rightarrow \pi_{f}^{\text {et }}\left(X_{k}\right)$. Similarly, there is a map $\pi_{f}^{\text {ét }} X_{k} \rightarrow \pi_{f}^{\text {ét }}(\operatorname{Spec} k)=\operatorname{Gal}(\bar{k} / k) \cong \widehat{\mathbb{Z}}$. Denote the canonical generator of $\operatorname{Gal}(\bar{k} / k)$ by $F$, the geometric Frobenius automorphism.

In constructing fundamental groupoids and étale homotopy types, we may use the same set of geometric points for both $X_{k}$ and $X$, so assume that $\alpha$ is an isomorphism on objects. We then have

$$
\pi_{f}^{\text {ét }}(X)=\pi_{f}^{\text {ét }}\left(X_{k}\right) \times_{\widehat{\mathbb{Z}}} 0 .
$$

Definition 6.1 Define the Weil groupoid $W_{f}\left(X_{k}\right)$ by

$$
W_{f}\left(X_{k}\right):=\pi_{f}^{\mathrm{e} t}\left(X_{k}\right) \times_{\widehat{\mathbb{Z}}} \mathbb{Z},
$$

noting that this is a pro-groupoid with discrete objects.

For any scheme $Y$, note that finite-dimensional representations of $\varpi_{f}^{\text {ét }}(Y):=\varpi_{f}\left(\widehat{Y_{\mathrm{et}}}\right)$ correspond to smooth $\mathbb{Q}_{\ell}$-sheaves on $Y$. We now introduce natural quotients of this groupoid.

Definition 6.2 Define $W_{\varpi_{f}}^{\text {ét }}(X)$ to be the image of the map $\varpi_{f}^{\text {ét }}(X) \rightarrow W_{f}\left(X_{k}\right)^{\text {alg }}$, so $W_{f}\left(X_{k}\right)^{\mathrm{alg}}=W_{\varpi_{f}^{\text {ét }}}(X) \ltimes \mathbb{Z}^{\mathrm{alg}}$.

Define ${ }^{\mathrm{Ga}} \varpi_{f}^{\text {ét }}(X)$ to be the image of the map $\varpi_{f}^{\text {ét }}(X) \rightarrow \varpi_{f}^{\text {ét }}\left(X_{k}\right)$, so $\varpi_{f}^{\text {ét }}\left(X_{k}\right)=$ ${ }^{\mathrm{Ga}} \varpi_{f}^{\text {ét }}(X) \ltimes \widehat{\mathbb{Z}}^{\mathrm{alg}}$. Note ${ }^{\mathrm{Ga}} \varpi_{f}^{\text {ét }}(X)$ is a Frobenius-equivariant quotient of $W_{\varpi_{f}^{\text {et }}}(X)$ (it is in fact the quotient on which $\widehat{\mathbb{Z}}$ acts continuously). 
In [38], $W_{\varpi_{1}}(X, \bar{x})$ was defined to be the universal object classifying continuous $W\left(X_{k}, x\right)$-equivariant homomorphisms $\pi_{1}(X, \bar{x}) \rightarrow G\left(\mathbb{Q}_{\ell}\right)$ to algebraic groups. In the terminology of [38, Definition 1.3], ${ }_{\varpi_{1}}(X, \bar{x})$ is the maximal quotient of $\varpi_{1}(X, \bar{x})$ on which Frobenius acts algebraically.

Note that these definitions are consistent by [38, Lemma 1.14], which proceeds by establishing an action of $\mathbb{Z}^{\text {alg }}$ on $W_{\varpi_{1}}(X, \bar{x})$ generated by Frobenius, then showing that the map $\mathbb{Z}^{\text {alg }} \ltimes W_{\varpi_{1}}(X, \bar{x}) \rightarrow W\left(X_{k}, x\right)^{\text {alg }}$ is an isomorphism.

It also implies that linear representations of $W_{\varpi_{f}^{\text {et }}}(X)$ correspond to smooth $\mathbb{Q}_{\ell}-$ sheaves on $X$ arising as subsheaves of Weil sheaves, while linear representations of ${ }^{\mathrm{Ga}} \varpi_{f}^{\text {ét }}(X)$ correspond to smooth $\mathbb{Q}_{\ell}$-sheaves on $X$ arising as subsheaves of pullbacks of smooth $\mathbb{Q}_{\ell}$-sheaves on $X_{k}$.

Lemma 6.3 The canonical action of $F$ on $W_{\varpi_{f}^{e}}$ é $(X)$ factors through a morphism

$$
\mathbb{Z}^{\mathrm{alg}} \rightarrow \operatorname{Aut}\left({ }^{W}{ }_{f}^{\text {ét }}(X)\right)
$$

of group presheaves, for $\mathbb{Z}^{\text {alg }}$ as in Section 5.1.

Proof Write $G=W_{\varpi_{f}^{\text {ét }}}(X), H=W_{f}\left(X_{k}\right)^{\mathrm{alg}}$, and observe that the orbits of $F$ in $\mathrm{Ob} G=\mathrm{Ob} H$ are finite, giving a map

$$
\widehat{\mathbb{Z}} \rightarrow \operatorname{Aut}(\mathrm{Ob} H) \text {. }
$$

Since $\widehat{\mathbb{Z}}$ is pro-finite, we may regard it as the pro-algebraic group $\mathbb{Z}^{\text {alg }} / \mathbb{Z}^{\text {alg,0 }}$.

Now, consider the group scheme

$$
N:=\coprod_{f \in \operatorname{Aut}(\mathrm{Ob}(H))} \prod_{x \in \mathrm{Ob}(H)} H(x, f x),
$$

with multiplication given by

$$
\left(f,\left\{h_{x}\right\}\right) \cdot\left(f^{\prime},\left\{h_{x}^{\prime}\right\}\right)=\left(f \cdot f^{\prime},\left\{h_{f^{\prime} x} \cdot h_{x}\right\}\right) .
$$

There is a morphism $N \rightarrow \operatorname{Aut}(\mathrm{Ob}(H))$ fibred in affine schemes. Thus

$$
\widehat{\mathbb{Z}} \times \operatorname{Aut}(\mathrm{Ob}(H))
$$

is an affine scheme.

Now, $F$ gives a collection of paths $F(x) \in W_{f}\left(X_{k}\right)(x, F x)$, and thus a map

$$
\mathbb{Z} \rightarrow(\widehat{\mathbb{Z}} \times \operatorname{Aut}(\mathrm{Ob}(H)) N)\left(\mathbb{Q}_{\ell}\right) .
$$


Since the latter is an affine group scheme, this extends to a map $\mathbb{Z}^{\text {alg }} \rightarrow \widehat{\mathbb{Z}} \times \operatorname{Aut}(\mathrm{Ob}(H)) N$. Finally, observe that the conjugation action of $H$ on $G$ gives a map

$$
N \rightarrow \operatorname{Aut}(G) \text {. }
$$

Theorem 6.4 If $X_{k} / k$ is normal, then the action of $\mathbb{Z}^{\text {red }}$ on $W_{\varpi_{f}^{\text {et }}}(X)^{\text {red }}$ factors through $P_{q}$ (see Definition 5.2), that is, the Frobenius representation $O\left({ }^{W_{\varpi}}{ }_{f}^{\text {ét }}(X)^{\text {red }}\right)$ is a sum of finite-dimensional Galois representations, pure of weight 0.

Moreover $W_{\varpi_{f}^{\text {ét }}}(X)^{\text {red }}={ }^{\mathrm{Ga}} \varpi_{f}^{\text {ét }}(X)^{\text {red }}$, so the $\mathbb{Z}^{\text {red }}$ action factors through its quotient $\widehat{\mathbb{Z}}^{\text {red }}$.

Proof Since $\mathbb{Z}^{\text {alg }}=\mathbb{Z}^{\text {red }} \times \mathbb{G}_{a}$ (Section 5.1), this amounts to showing that the Frobenius action factors through $P_{q} \times \mathbb{G}_{a}$. We adapt the proof of [38, Theorem 1.11] (to which we refer the reader for details).

Let $T$ be the set of all isomorphism classes of irreducible representations $V$ of $W_{\varpi_{f}^{e}}{ }_{\text {et }}(X)^{\text {red }}$ over $\overline{\mathbb{Q}_{\ell}}$. Since $W_{\varpi_{f}^{\text {et }}}(X)^{\text {red }}$ is reductive, there is an isomorphism of $W_{\varpi_{f}^{\text {et }}}(X)^{\mathrm{red}} \times W_{\varpi_{f}^{\text {et }}}(X)^{\mathrm{red}}$-representations given on objects $(x, y)$ by

$$
O\left({ }^{W} \varpi_{f}^{\mathrm{et}}(X)^{\mathrm{red}}(x, y)\right) \otimes_{\mathbb{Q}_{\ell}} \overline{\mathbb{Q}_{\ell}} \cong \bigoplus_{V \in T} \operatorname{Hom}\left(V_{x}, V_{y}\right)
$$

Suppose $\mathbb{V}$ is the smooth sheaf on $X$ corresponding to the representation $V$. Then $\bigoplus_{V \in T} \operatorname{Hom}\left(V_{x}, V_{y}\right)$ corresponds to the smooth sheaf

$$
\bigoplus_{V \in T} \mathrm{pr}_{1}^{-1} \mathbb{V}^{\vee} \otimes \mathrm{pr}_{2}^{-1} \mathbb{V}
$$

on $X \times X$.

Now, $V \in T$ is an irreducible representation of $\varpi_{f}^{\text {ét }}(X)^{\text {red }}$ which is a subrepresentation of some $W_{f}\left(X_{k}\right)$-representation. This is the same as underlying a $W_{f}\left(X_{k^{\prime}}\right)$ representation for some finite extension $k^{\prime} / k$, so $\mathbb{V}$ underlies a smooth Weil sheaf on $X_{k^{\prime}}$.

From Lafforgue's Theorem ([5, Conjecture 1.2.10], proved in [27, Theorem VII.6 and Corollary VII.8]), every irreducible smooth Weil sheaf over $\overline{\mathbb{Q}_{\ell}}$ is of the form

$$
\mathbb{V} \cong P \otimes \overline{\mathbb{Q} \ell}^{(b)}
$$

for some mixed sheaf $P$ on $X_{k^{\prime}}$. By [5, Theorem 3.4.1 (ii)], every irreducible smooth $\iota$-mixed Weil sheaf is $\iota$-pure. Thus the mixed sheaf $P$ is $\iota$-pure for all $\iota$, and hence pure.

Thus

$$
\mathrm{pr}_{1}^{-1} \mathbb{V}^{\vee} \otimes \mathrm{pr}_{2}^{-1} \mathbb{V} \cong \mathrm{pr}_{1}^{-1} P^{\vee} \otimes \mathrm{pr}_{2}^{-1} P,
$$

which is a smooth sheaf on $X_{k^{\prime}} \times X_{k^{\prime}}$, pure of weight 0 . 
Hence $O\left({ }^{W_{\varpi_{f}} \text { ét }}(X)^{\text {red }}\right) \otimes_{\mathbb{Q}_{\ell}} \overline{\mathbb{Q}_{\ell}}$, and hence $O\left({ }^{W_{\varpi}}\right.$ ét $\left.(X)^{\text {red }}\right)$, is a pure Galois representation of weight 0 . Thus the action of $\mathbb{Z}^{\text {alg }}$ factors through $P_{q} \times \mathbb{G}_{a}$, and the discrete Galois action on $\varpi_{f}^{\text {et }}(X)^{\text {red }}$ descends to a continuous action on $W_{\varpi_{f}}^{\text {ét }}(X)^{\text {red }}$, so

$$
W_{\varpi_{f}}{ }^{\mathrm{e}}(X)^{\mathrm{red}}={ }^{\mathrm{Ga}} \varpi_{f}^{\mathrm{e}}(X)^{\mathrm{red}} .
$$

\subsection{Weight decompositions}

Now assume that $X$ is either smooth or proper and normal.

Definition 6.5 Define a weight decomposition on a multipointed homotopy type $G \in$ $\operatorname{Ho}\left(s \mathcal{E}(R)_{*}\right)$ to be a morphism

$$
\mathbb{G}_{m} \rightarrow \operatorname{RAut}(G)
$$

of pro-algebraic groups.

Compare this with [37, Definition 5.15], which considers weight decompositions on unpointed homotopy types, corresponding to outer automorphisms.

Proposition 6.6 If we let $R$ be any Frobenius-equivariant quotient of $W_{\varpi_{f}}^{\text {ét }}(X)^{\text {red }}$, then the Galois action on

$$
X_{\text {ét }}^{R, \mathrm{Mal}}
$$

is mixed, giving a canonical weight decomposition. Furthermore, the Frobenius action extends canonically to a continuous algebraic $\mathrm{Gal}(\bar{k} / k)$-action.

Proof By Theorem 6.4, the Galois action on $R$ factors through the quotient $P_{q} \times \mathbb{G}_{a}$ of $\mathbb{Z}^{\text {alg }}$. By Corollary 4.8 , the $\operatorname{Gal}(\bar{k} / k)$ action on $X_{\text {ét }}^{R \text {,Mal }}$ is thus algebraic. Since $R$ is a $P_{q} \times \mathbb{G}_{a}$-representation, the Weil sheaf $\bigoplus_{x \in \mathrm{Ob} R} \mathbb{O}(R)(x)$ is an arithmetic sheaf of weight 0 . Deligne's Weil II theorems [5, Corollaries 3.3.4-3.3.6] then imply that $\bigoplus_{x \in X} \mathrm{H}^{*}(X, \mathbb{O}(R)(x))$ is a mixed $\operatorname{Gal}(\bar{k} / k)$ representation (ie a representation of $M_{q} \times \mathbb{G}_{a}$ ). By Lemma 4.9, we may therefore conclude that the action of $\mathbb{Z}^{\text {red }}$ on $X_{\text {ét }}^{R, \text { Mal }}$ factors through $M_{q}$, giving

$$
M_{q} \rightarrow \operatorname{Aut}^{h}\left(X_{\text {et }}^{R, M a l}\right) .
$$

Finally, use the map $\mathbb{G}_{m} \rightarrow M_{q}$ (given after Definition 5.1) to define the weight decomposition. Since $R$ is pure of weight zero, the $\mathbb{G}_{m}$-action on $R$ is trivial, giving

$$
\mathbb{G}_{m} \rightarrow \operatorname{Aut}^{h}\left(X_{\text {ét }}^{R, \mathrm{Mal}}\right),
$$

as required. 
Corollary 6.7 The Galois actions are mixed on the duals $\varpi_{n}\left(X_{\text {ét }}^{R, \mathrm{Mal}}, x\right)^{\vee}$ of the homotopy groups for $n \geq 2$, and on the structure sheaves $O\left(\varpi_{f}\left(X_{\mathrm{et}}^{R, \mathrm{Mal}}\right)\right)(x, y)$. In particular, these objects have canonical weight decompositions.

Proof This is just the observation that there are canonical maps $\operatorname{Aut}^{h}\left(X_{\mathrm{et}}^{R, \mathrm{Mal}}\right) \rightarrow$ $\operatorname{Aut}\left(\varpi_{n}\left(X_{\text {ét }}^{R, \mathrm{Mal}}, x\right)\right)$ and $\operatorname{Aut}^{h}\left(X_{\text {ét }}^{R, \mathrm{Mal}}\right) \rightarrow \operatorname{Aut}\left(O\left(\varpi_{f}\left(X_{\text {ét }}^{R, \mathrm{Mal}}\right)\right)(x, y)\right)$ of groupvalued presheaves, so Proposition 6.6 gives algebraic actions of $M_{q} \times \mathbb{G}_{a}$ (and hence $\mathbb{G}_{m}$ ) on the homotopy groups and fundamental groupoid.

Remark 6.8 We have shown that $\varpi_{n}\left(X_{\text {ét }}^{R, \mathrm{Mal}}\right)$ is a mixed $\pi_{f}^{\text {ét }}\left(X_{k}\right)$-representation. In particular, this means that $\varpi_{n}\left(X_{\text {ét }}^{R, \text { Mal }}, \bar{x}\right)$ is a mixed $\left\langle\widehat{\left.F_{x}\right\rangle}\right.$-representation, so has a canonical weight decomposition.

Remark 6.9 If the hypotheses of Theorem 3.40 hold and $\pi_{1}^{\text {ét }}(X, x)$ is $N$-good relative to $R$, then Corollary 6.7 implies that the Galois actions on the $\pi_{n}^{\text {et }}(X, x) \otimes_{\widehat{\mathbb{Z}}} \mathbb{Q}_{\ell}$ are mixed for $n \leq N$.

Alternatively, if it should happen that the Galois action on $\mathrm{H}^{n}\left(\pi_{1}^{\text {ét }}(X, x), V\right)$ is mixed for all $R$-representations $V$ underlying pure $\pi_{1}^{\text {ét }}\left(X_{k}, x\right)$-representations and all $n \leq N$, then Lemma 4.9 (combined with the Adams spectral sequence of [37, Proposition 4.37]) implies that the Galois actions on $\varpi_{n}\left(B \pi_{1}(X, x)\right)^{R \text {, Mal }}$ is mixed for $n \leq N$. Provided the first two hypotheses of Theorem 3.40 hold, the exact sequence of that theorem would then imply that the Galois actions on $\pi_{n}^{\text {ét }}(X, x) \otimes_{\widehat{\mathbb{Z}}} \mathbb{Q}_{\ell}$ are also mixed.

\subsection{Formality}

Now assume that $X$ is smooth and proper. Deligne's Weil II theorems then imply that $\bigoplus_{x \in X} \mathrm{H}^{n}(X, \mathbb{O}(R)(x))$ is pure of weight $n$.

Theorem 6.10 For $R$ as in Proposition 6.6, the Malcev homotopy type $X_{\text {ét }}^{R, \text { Mal }} \in$ $s \mathcal{E}(R)$ is formal, in the sense that it corresponds (under the equivalences of Proposition 2.50 and Theorem 2.74) to the $R$-representation

$$
\mathrm{H}_{\mathrm{et}}^{*}(X, \mathbb{O}(R))
$$

in cochain algebras, equipped with the unique augmentation map $\mathbb{Q}_{\ell}=\mathrm{H}^{0}(X, \mathbb{O}(R)) \rightarrow$ $\prod_{x \in \mathrm{Ob} R} O(R)(x,-)$. This isomorphism is Galois equivariant.

Proof We need to construct an isomorphism $\theta: N \mathrm{R}_{\mathrm{u}}\left(X_{\text {ét }}^{\rho, \mathrm{Mal}}\right) \cong G \mathrm{H}_{\text {et }}^{*}(X, \mathbb{O}(R))$ in $d g \mathcal{P}(R)$ (for $G$ as in Definition 4.20), such that

$$
\operatorname{ad}_{\theta}: \operatorname{Aut}^{h}\left(X_{\mathrm{et}}^{\rho, \mathrm{Mal}}\right) \rightarrow \operatorname{Aut}^{h}\left(G \operatorname{Spec} D \mathrm{H}_{\mathrm{et}}^{*}(X, \mathbb{O}(R)) \rtimes R\right)
$$

satisfies $\operatorname{ad}_{\theta} F=F$. 
As in Section 4.3.4, take a minimal model $\mathfrak{m}$ for $N \mathrm{R}_{\mathrm{u}}\left(X_{\text {ét }}^{\rho, \mathrm{Mal}}\right) \in d g \hat{\mathcal{N}}(R)$. This has the property that $\mathfrak{m}_{n} /[\mathfrak{m}, \mathfrak{m}]_{n} \cong \mathrm{H}^{n+1}(X, \mathbb{O}(R))^{\vee}$.

From the proof of Lemma 4.4, we know that

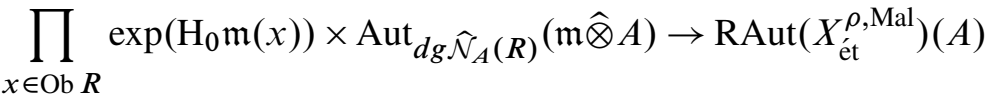

is a pro-unipotent extension of pro-algebraic groups.

Likewise, the maps

$$
\begin{aligned}
\operatorname{Aut}(R \ltimes \exp (\mathfrak{m})) & \rightarrow \operatorname{Aut}^{h}\left(X_{\mathrm{et}}^{\rho, \mathrm{Mal}}\right) \\
& \rightarrow\left\{(f, \alpha): f \in \operatorname{Aut}(R), \alpha \in \operatorname{Iso}_{D G \operatorname{Alg}(R)}\left(\mathrm{H}_{\mathrm{et}}^{*}(X, \mathbb{O}(R)), f^{\sharp} \mathrm{H}_{\mathrm{et}}^{*}(X, \mathbb{O}(R))\right)\right\}
\end{aligned}
$$

both have pro-unipotent kernels.

So we may lift the map $\widehat{\mathbb{Z}}^{\text {alg }} \rightarrow \operatorname{Aut}^{h}\left(X_{\text {ét }}^{\rho, \text { Mal }}\right)$ to give $\widehat{\mathbb{Z}}^{\text {alg }} \rightarrow \operatorname{Aut}(R \ltimes \exp (\mathfrak{m}))$. This gives a lift of the weight decomposition $\mathbb{G}_{m} \rightarrow \operatorname{RAut}\left(X_{\text {ét }}^{\rho, \mathrm{Mal}}\right)$ to $\mathbb{G}_{m} \rightarrow \operatorname{Aut}(R \ltimes \exp (\mathfrak{m}))$. Since $\mathfrak{m}$ is of strictly negative weights, we may adapt [38, Corollary 1.21$]$ by observing that $O(R \ltimes \exp (\mathfrak{m})) / O(R)$ is of strictly positive weights, and that the weight 0 part $\mathcal{W}_{0} O(R \ltimes \exp (\mathfrak{m}))$ is just $\mathrm{O}(\mathrm{R})$, so we have a $\widehat{\mathbb{Z}}^{\text {alg }}$-equivariant decomposition

$$
O(R \ltimes \exp (\mathfrak{m}))=O(R) \oplus \mathcal{W}_{+} O(R \ltimes \exp (\mathfrak{m})) .
$$

This amounts to giving a $\widehat{\mathbb{Z}}^{\text {alg }}$-equivariant section of $R \ltimes \exp (\mathfrak{m}) \rightarrow R$, or equivalently a $\widehat{\mathbb{Z}}^{\text {alg }}$-equivariant Levi decomposition, so we may assume that the $\widehat{\mathbb{Z}}^{\text {alg }}$ action on $R \ltimes \exp (\mathfrak{m})$ consists of actions on $R$ and on $\mathfrak{m}$.

Let $V_{n}:=\mathcal{W}_{-n-1} \mathfrak{m}_{n}$, for $\mathcal{W}$ as in Section 5.1; since cohomology is pure, we deduce that $V_{n} \rightarrow \mathrm{H}^{n+1}(X, \mathbb{O}(R))^{\vee}$ is an isomorphism, and that $\mathfrak{m}$ is freely generated as a Lie algebra by the spaces $V_{n}$. The differential $d$ on $\mathfrak{m}$ is then determined by $d: V_{n} \rightarrow \mathfrak{m}_{n-1}$, and weight considerations show that the only nonzero contribution is $V_{n} \rightarrow \prod_{a+b=n-1}\left[V_{a}, V_{b}\right]$. This is isomorphic to $d: \mathfrak{m} /[\mathfrak{m}, \mathfrak{m}] \rightarrow[\mathfrak{m}, \mathfrak{m}] /[\mathfrak{m},[\mathfrak{m}, \mathfrak{m}]]$, so must be dual to the cup product.

Therefore, the choice of lift $\widehat{\mathbb{Z}}^{\text {alg }} \rightarrow \operatorname{Aut}(R \ltimes \exp (\mathfrak{m}))$ has determined an isomorphism $R \ltimes \exp (\mathfrak{m}) \cong R \ltimes \exp \left(G \mathrm{H}_{\mathrm{et}}^{*}(X, \mathbb{O}(R))\right)$, and this is automatically compatible with the Galois action $\widehat{\mathbb{Z}}^{\text {alg }} \rightarrow \operatorname{Aut}^{h}(R \ltimes \exp (\mathfrak{m}))$.

Corollary 6.11 If we let $R$ be any Frobenius-equivariant quotient of $W_{\varpi_{f}^{\text {et }}}(X)^{\mathrm{red}}$, then the relative Malcev homotopy groups $\varpi_{n}^{\text {ét }}\left(X^{R, \text { Mal }}, x\right)$ can be described in terms of cohomology as

$$
\varpi_{n}^{\text {ét }}\left(X^{R, \mathrm{Mal}}, x\right) \cong \mathrm{H}_{n-1}\left(G \mathrm{H}^{*}(X, \mathbb{O}(R))\right),
$$


for $G$ as in Definition 4.20. This description is Galois-equivariant. If the conditions of Theorem 3.40 hold (including goodness), then this also calculates $\pi_{n}^{\text {ét }}(X, x) \otimes_{\widehat{\mathbb{Z}}} \mathbb{Q}_{\ell}$ as a Galois representation.

\subsection{Quasiformality}

Let $j: X \hookrightarrow \bar{X}$ be an open immersion of varieties over $\bar{k}$, such that locally for the étale topology, the pair $(X, \bar{X})$ is isomorphic to $\left(\mathbb{A}^{m} \times \prod_{i}\left(\mathbb{A}^{c_{i}}-\{0\}\right), \mathbb{A}^{d}\right)$, for some $d=m+\sum c_{i}$. Note that this is satisfied when $\bar{X}-X$ is a normal crossings divisor (corresponding to the case $c_{i}=1$ for all $i$ ). It also includes all geometric fibrations over $\bar{k}$ in the sense of [10, Definition 11.4].

Definition 6.12 For $X, \bar{X}$ as above, let $T=\bar{X}-X$, and let $D$ be the closed subscheme of $T$ of codimension 1 in $\bar{X}$. Note that $\pi_{f}^{\text {et }}(X) \rightarrow \pi_{f}^{\text {et }}(\bar{X}-D)$ is an isomorphism, and define $\pi_{f}^{t}(X):=\pi_{f}^{t}(\bar{X}-D)$ to be the tame fundamental groupoid (as in [12, XIII.2.1.3]).

Define $\pi_{f}^{t}\left(X_{k}\right)$ similarly, with the tame Weil groupoid $W_{f}^{t}\left(X_{k}\right)$ given by

$$
W_{f}^{t}\left(X_{k}\right):=\pi_{f}^{t}\left(X_{k}\right) \times_{\widehat{\mathbb{Z}}} \mathbb{Z} .
$$

Let $\varpi_{f}^{t}(X):=\pi_{f}^{t}(X)^{\mathrm{alg}}$, and define ${ }^{W_{\varpi_{f}}^{t}(X)}$ to be the image of $\varpi_{f}^{t}(X) \rightarrow W_{f}^{t}\left(X_{k}\right)^{\mathrm{alg}}$. Given a local system $\mathbb{V}$ on $X$, observe that the direct image $i_{*} \mathbb{V}$ of $\mathbb{V}$ under the inclusion $i: X \hookrightarrow \bar{X}-D$ is also a local system. We say that $\mathbb{V}$ is tamely ramified along the divisor if $i_{*} \mathbb{V}$ is tamely ramified along $D$ in the sense of [12, Definition XIII.2.1.1].

Lemma 6.13 Take $j$ as above. If $\mathbb{V}$ is a pure smooth Weil sheaf on $Y$ of weight zero, tamely ramified along the divisor, then $\mathbf{R}^{v} j_{*} \mathbb{V}$ is pure of weight $2 v$ (in the sense of [25, Lemma-Definition II.12.7]).

Proof This is a consequence of the following statements:

(1) $\mathbf{R}^{v} j_{*} \mathbb{V}$ is pointwise pure of weight $2 v$.

(2) the canonical map $\left(\mathbf{R}^{v} j_{*} \mathbb{V}\right)^{\vee} \rightarrow \mathbf{R} \operatorname{Hom}_{\bar{X}}\left(\mathbf{R}^{v} j_{*} \mathbb{V}, \mathbb{Q}_{\ell}\right)$ is an isomorphism.

If $0 \rightarrow \mathbb{V}^{\prime} \rightarrow \mathbb{V} \rightarrow \mathbb{V}^{\prime \prime} \rightarrow 0$ is an exact sequence, with the statements holding for $\mathbb{V}$ and $\mathbb{V}^{\prime \prime}$, then observe that they also hold for $\mathbb{V}$, since the long exact sequence must degenerate.

The statements are local on $\bar{X}$. Étale-locally, the pair $(X, \bar{X})$ is isomorphic to $\left(U, U^{\prime}\right)=\left(\mathbb{A}^{m} \times \prod_{i}\left(\mathbb{A}^{c_{i}}-\{0\}\right), \mathbb{A}^{d}\right)$, for $d=m+\sum c_{i}$. We may then reduce to the 
case when $\mathbb{V}$ is irreducible on $U$, and so $\mathbb{V}=\mathbb{V}_{m} \otimes \bigotimes_{i} \mathbb{V}_{i}$, for $\mathbb{V}_{i}$ irreducible on $\mathbb{A}^{c_{i}}-\{0\}$. By the Künneth formula, we now need only consider the pair $\left(\mathbb{A}^{c}-\{0\}, \mathbb{A}^{c}\right)$.

If $\mathbb{V}$ is constant, then the statements follow from the cohomological purity theorem [30, VI.5.1]. Since the scheme $\mathbb{A}^{c}-\{0\}$ is simply connected for $c>1$, this leaves only the case $c=1$. [25, Lemma I.9.1] shows that $j_{*} \mathbb{V}$ is pure, and local calculations give $\mathbf{R}^{i} j_{*} \mathbb{V}=0$ for $i>0$ (since $\mathbb{V}$ is tamely ramified, and is nonconstant irreducible).

Proposition 6.14 Assume that $j: X_{k} \hookrightarrow \bar{X}_{k}$ is a morphism over $k$, with $j \otimes \bar{k}$ as in Lemma 6.13, for $\bar{X}_{k}$ proper. If $\mathbb{V}$ is a pure smooth Weil sheaf on $X$ of weight zero, tamely ramified along the divisor, then $\mathrm{H}^{i}\left(\bar{X}, \mathbf{R}^{v} j_{*} \mathbb{V}\right)$ is pure of weight $i+2 v$, for $j: X \rightarrow \bar{X}$ the compactification map.

Proof By [5, Corollary 3.3.4], we know that $\mathrm{H}^{i}\left(\bar{X}, \mathbf{R}^{v} j_{*} \mathbb{V}\right)$ is mixed of weights $\leq i+2 v$, since $\mathbf{R}^{v} j_{*} \mathbb{V}$ is pure of weight $2 v$. Now, Poincaré duality [25, Corollary II.7.3] implies that

$$
\mathrm{H}^{i}\left(\bar{X}, \mathbf{R}^{v} j_{*} \mathbb{V}\right)^{\vee} \cong \mathrm{H}^{2 d-i}\left(\bar{X},\left(\mathbf{R}^{v} j_{*} \mathbb{V}\right)^{\vee}\right)(2 d),
$$

which is mixed of weight $\leq-i-2 v$, using the isomorphism

$$
\left(\mathbf{R}^{v} j_{*} \mathbb{V}\right)^{\vee} \cong \mathbf{R} \operatorname{Hom}_{\bar{X}}\left(\mathbf{R}^{v} j_{*} \mathbb{V}, \mathbb{Q}_{\ell}\right)
$$

of Lemma 6.13.

Corollary 6.15 For $X$ as above, and $\rho: \varpi_{f}^{\text {ét }} X \rightarrow R$ any Frobenius-equivariant quotient of $W_{\varpi_{f}^{t}}(X)^{\mathrm{red}}$, the filtered homotopy type $\left(X^{\rho, \mathrm{Mal}}, j\right)$ of Definition 4.37 is quasiformal (in the sense of Definition 4.24). The formality quasi-isomorphism is equivariant with respect to the Galois action.

Proof This is largely the same as Theorem 6.10. Use the equivalences of Theorem 4.22 to take a filtered minimal model $(\mathfrak{m}, J) \in F s \hat{\mathcal{N}}(R)$ for $\left(X^{\rho, \text { Mal }}, j\right)$. The increasing filtration $J_{*}$ on $\mathfrak{m}^{\vee}$ gives a decreasing filtration $J^{*}$ on $\mathfrak{m}$, with $J^{r} \mathfrak{m}_{n}$ the annihilator of $J_{r-1}\left(\mathfrak{m}^{\vee}\right)$. Note that $\left[J^{a} \mathfrak{m}, J^{b} \mathfrak{m}\right] \subset J^{a+b} \mathfrak{m}$ and $J^{0} \mathfrak{m}=\mathfrak{m}$.

If we write $\operatorname{Aut}_{J}(R \ltimes \exp (\mathfrak{m}))$ for the group of filtered automorphisms of $R \ltimes \exp (\mathfrak{m})$, then similarly to Lemma 4.31, the maps

$\operatorname{Aut}_{J}(R \ltimes \exp (\mathfrak{m})) \rightarrow \operatorname{Aut}_{J}^{h}\left(X_{\mathrm{et}}^{\rho, \mathrm{Mal}}\right) \rightarrow$

$\left\{(f, \alpha): f \in \operatorname{Aut}(R), \alpha \in \operatorname{Iso}_{F D G} \operatorname{Alg}(R)\left(\mathrm{H}_{\mathrm{et}}^{*}\left(\bar{X}, \mathbf{R}^{*} j_{*} \mathbb{O}(R)\right), f^{\sharp} \mathrm{H}_{\mathrm{et}}^{*}\left(\bar{X}, \mathbf{R}^{*} j_{*} \mathbb{O}(R)\right)\right)\right\}$

both have pro-unipotent kernels. 
We may therefore lift the Galois action $\widehat{\mathbb{Z}}^{\text {alg }} \rightarrow \operatorname{Aut}_{J}^{h}\left(X_{\mathrm{et}}^{\rho, \mathrm{Mal}}\right)$ to a filtered automorphism of $R \ltimes \exp (\mathfrak{m})$. This gives a lift of the weight decomposition $\mathbb{G}_{m} \rightarrow \operatorname{RAut}_{J}\left(X_{\mathrm{et}}^{\rho, \mathrm{Mal}}\right)$, a unique Galois-equivariant Levi decomposition of $R \ltimes \exp (\mathfrak{m})$, and a weight decomposition $\mathbb{G}_{m} \rightarrow \operatorname{Aut}_{J}(\mathfrak{m})$.

Now, $\left(\mathfrak{m}_{n}^{\mathrm{ab}}\right)^{\vee} \cong \bigoplus_{a+b=n+1} \mathrm{H}^{a}\left(\bar{X}, \mathbf{R}^{b} j_{*} \mathbb{O}(R)\right)=: E^{n+1}$, on which $J_{r}$ is the subspace of weights $\leq n+r+1$. Thus $J^{r}\left(\mathfrak{m}_{n}^{\mathrm{ab}}\right)$ is the subspace of weights $\leq-(n+r+1)$.

Let $\Gamma^{r} \mathfrak{m}$ be the lower central series on $\mathfrak{m}$, so $\Gamma^{1} \mathfrak{m}=\mathfrak{m}$ and $\Gamma^{r+1} \mathfrak{m}=\left[\mathfrak{m}, \Gamma^{r} \mathfrak{m}\right]$. The weight restrictions on $\mathfrak{m}^{\mathrm{ab}}$ show that $J^{r}\left(\operatorname{gr}_{\Gamma}^{s} \mathfrak{m}\right)_{n}=J^{r}\left(\operatorname{Lie}_{s}\left(\mathfrak{m}^{\mathrm{ab}}\right)\right)_{n}$, which is of weights $\leq-(n+r+s)$. This implies that $J^{r}\left(\Gamma_{s} \mathfrak{m}\right)_{n}$ is of weights $\leq-(n+r+s)$.

We now make a canonical choice of generators by setting

$$
\mathcal{W}_{-(n+r+1)} V_{n}:=\mathcal{W}_{-(n+r+1)} J^{r} \mathfrak{m}_{n}
$$

Set $V:=\prod_{i} \mathcal{W}_{i} V$; the weight conditions above show that this has no intersection with $\Gamma_{s} \mathfrak{m}$ for $s>1$, so the composition $V \rightarrow \mathfrak{m} \rightarrow \mathfrak{m}^{\text {ab }}$ is injective. Since $\mathcal{W}_{-(n+r+1)}\left(\mathfrak{m}^{\mathrm{ab}}\right)_{n}=\mathcal{W}_{-(n+r+1)} J^{r}\left(\mathfrak{m}^{\mathrm{ab}}\right)_{n}$, the composition is also surjective, so $V$ is a space of generators for $\mathfrak{m}$.

The structure of $\mathfrak{m}$ is now determined by the differentials $d: V_{n} \rightarrow \mathfrak{m}_{n-1}$. As $\mathfrak{m}=$ $\operatorname{Lie}(V)=V \times \bigwedge^{2} V \times \Gamma_{3} \mathfrak{m}$, weight and filtration considerations show that we must have the projection $d: V_{n} \rightarrow\left(\Gamma_{3} \mathfrak{m}\right)_{n-1}$ being 0 . The nonzero contributions to $d$ are $V_{n} \rightarrow V_{n-1}$, which is dual to $d_{1}$ on $E$, and $V_{n} \rightarrow \prod_{a+b=n-1}\left[V_{a}, V_{b}\right]$, which must be dual to the cup product. Thus $\mathfrak{m}=G(E)$, and so $R \ltimes \exp (\mathfrak{m})=R \ltimes \exp (G(E))$, as required.

Corollary 6.16 For $X$ and $R$ as above, we can describe the relative Malcev homotopy groups $\varpi_{n}^{\text {et }}\left(X^{R, \mathrm{Mal}}, x\right)$ explicitly in terms of the Leray spectral sequence as

for

$$
\begin{gathered}
\mathrm{H}_{n-1}\left(G\left(J \mathrm{E}_{1}^{*, *}\right)\right), \\
{ }_{J} \mathrm{E}_{1}^{a, b}=\mathrm{H}^{2 a+b}\left(\bar{X}, \mathbf{R}^{-a} j_{*} \mathbb{O}\left({ }^{W} \varpi_{f}^{\text {ét }}(X)^{L, \mathrm{red}}\right)\right)
\end{gathered}
$$

as in Definition 4.11, and $G$ as in Definition 4.20. If the conditions of Theorem 3.40 hold (including goodness), then this also calculates $\pi_{n}^{\text {ét }}(X, x) \otimes_{\widehat{\mathbb{Z}}} \mathbb{Q}_{\ell}$ as a Galois representation. 


\section{Varieties over local fields}

\subsection{Potentially good reduction, $\ell \neq p$}

Let $V^{\prime}$ be a complete discrete valuation ring, with residue field $k^{\prime}$ (finite, of characteristic $p \neq \ell$ ), and fraction field $K^{\prime}$ (of characteristic 0 ). Let $\bar{k}, \bar{K}$ be the algebraic closures of $k, K^{\prime}$ respectively, and $\bar{V}$ the algebraic closure of $V^{\prime}$ in $\bar{K}$.

Let $X_{V^{\prime}}=\bar{X}_{V^{\prime}}-T_{V^{\prime}}$ be a geometric fibration over $V^{\prime}$ (in the sense of [10, Definition 11.4]). Assume that we have a subfield $K \subset K^{\prime}$ and a scheme $X_{K} / K$ such that $X_{K} \otimes_{K} K^{\prime} \cong X_{V^{\prime}} \otimes_{V^{\prime}} K^{\prime}$. We wish to study the $\operatorname{Gal}(\bar{K} / K)$-action on the homotopy type $X_{\bar{K} \text {,ét }}$.

Recall from [12, Theorem X.2.1] that the map $\pi_{f}^{\text {ét }}\left(\bar{X}_{k^{\prime}}\right) \rightarrow \pi_{f}^{\text {ét }}\left(\bar{X}_{V^{\prime}}\right)$ is an equivalence. By ibid. Section XIII.2.10, this generalises to an equivalence $\pi_{f}^{t}\left(X_{k^{\prime}}\right) \rightarrow \pi_{f}^{t}\left(X_{V^{\prime}}\right)$. Meanwhile, ibid. Corollary XIII.2.8 implies that the map $\pi_{f}^{t}\left(X_{\bar{K}}\right) \rightarrow \pi_{f}^{t}\left(X_{\bar{V}}\right)$ is an epimorphism, and ibid. Corollary XIII.2.9 shows $\pi_{f}^{\text {ét }}\left(X_{\bar{K}}\right)^{\wedge_{L}} \rightarrow \pi_{f}^{\text {ét }}\left(X_{\bar{V}}\right)^{\wedge_{L}}$ is an equivalence, where $L$ is any set of prime numbers excluding $p$.

Proposition 7.1 If $\mathbb{V}$ is an $\ell$-adic local system on $X_{\bar{V}}$, tamely ramified along the divisor (ie coming from a representation of $\pi_{f}^{t}\left(X_{\bar{V}}\right)$ ), then the maps

$$
\begin{aligned}
& i_{\eta}^{*}: \mathrm{H}^{*}\left(X_{\bar{V}}, \mathbb{V}\right) \rightarrow \mathrm{H}^{*}\left(X_{\bar{K}}, i_{\eta}^{*} \mathbb{V}\right) \\
& i_{s}^{*}: \mathrm{H}^{*}\left(X_{\bar{V}}, \mathbb{V}\right) \rightarrow \mathrm{H}^{*}\left(X_{\bar{k}}, i_{s}^{*} \mathbb{V}\right)
\end{aligned}
$$

are isomorphisms.

Proof In [10, Theorem 11.5], this is proved for $\pi_{f}^{\text {ét }}\left(X_{\bar{V}}\right)^{\wedge} L$-representations, for $p \notin L$. The same proof carries over to $\pi_{f}^{t}\left(X_{\bar{V}}\right)$-representations, since the pro- $L$ hypothesis is only used to restrict the monodromy around the divisor.

Definition 7.2 Since $\pi_{1}^{\text {ét }}\left(\operatorname{Spec} V^{\prime}\right) \cong \operatorname{Gal}\left(\bar{k} / k^{\prime}\right)$, we may define $W_{\varpi_{f}^{t}}\left(X_{\bar{V}}\right)$ analogously to Definition 6.2 as the maximal quotient of $\varpi_{f}^{t}\left(X_{\bar{V}}\right):=\pi_{f}^{t}\left(X_{\bar{V}}\right)^{\text {alg }}$ on which the Frobenius action is algebraic. Define ${ }^{\mathrm{pnr}} \varpi_{f}^{t}\left(X_{\bar{K}}\right)$ to be the image of $\varpi_{f}^{t}\left(X_{\bar{K}}\right) \rightarrow W_{\varpi_{f}^{t}}\left(X_{\bar{V}}\right)$, noting that this is a quotient of $\varpi_{f}^{t}\left(X_{\bar{K}}\right)$ on which the $\mathrm{Gal}(\bar{K} / K)$-action is potentially unramified.

Note that these definitions are independent of the choice of extension $V^{\prime} / V$, in the sense that a finite extension $V^{\prime \prime} / V^{\prime}$ would give the same construction. 
Theorem 7.3 Let $R$ be any Frobenius-equivariant reductive quotient of ${ }^{\mathrm{pnr}} \varpi_{f}^{t}\left(X_{\bar{K}}\right)$. Then the $\operatorname{Gal}(\bar{K} / K)$-action on the homotopy type

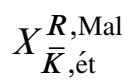

is algebraic, potentially unramified (as in Section 5.2) and mixed (Definition 5.16), giving a canonical Galois-equivariant weight decomposition. It is also quasiformal, corresponding to the $E_{2}$-term

$$
\bigoplus_{a, b} \mathrm{H}^{a}\left(\bar{X}_{\bar{K}}, \mathbf{R}^{b} j_{*} \mathbb{O}(R)\right) \in F D G \operatorname{Alg}(R),
$$

of the Leray spectral sequence for the immersion $j: X \rightarrow \bar{X}$. The formality quasiisomorphism is equivariant with respect to the $\operatorname{Gal}(\bar{K} / K)$-action.

Proof We know that the homotopy type is given by

$$
\mathrm{C}_{\mathrm{et}}^{\bullet}\left(X_{\bar{K}}, \mathbb{O}(R)\right) \in c \operatorname{Alg}(R) \text {. }
$$

From the definition of ${ }^{\mathrm{pnr}} \varpi_{f}^{t}\left(X_{\bar{K}}\right)$, we know that $\mathbb{O}(R)$ is the pullback of a local system on $X_{\bar{V}}$, so $i_{\eta *} \mathbb{O}(R)$ is a local system and $i_{\eta}^{*} i_{\eta *} \mathbb{O}(R)=\mathbb{O}(R)$.

The equivalences of Proposition 7.1 now give quasi-isomorphisms

$$
\begin{aligned}
\mathrm{C}_{\text {êt }}^{\bullet}\left(X_{\bar{K}}, \mathbb{O}(R)\right)=\mathrm{C}_{\text {et }}^{\bullet}\left(X_{\bar{K}}, i_{\eta}^{*} i_{\eta *} \mathbb{O}(R)\right) & \\
& \leftarrow \mathrm{C}_{\text {et }}^{\bullet}\left(X_{\bar{V}}, i_{\eta *} \mathbb{O}(R)\right) \rightarrow \mathrm{C}_{\text {et }}^{\bullet}\left(X_{\bar{k}}, i_{s}^{*} i_{\eta *} \mathbb{O}(R)\right),
\end{aligned}
$$

compatible with the basepoint augmentation maps.

We may assume that $K \subset K^{\prime}$ is a Galois extension, then observe that the equivalences above imply that action of $\operatorname{Gal}\left(\bar{K} / K^{\prime}\right)$ is unramified, so the $\operatorname{Gal}(\bar{K} / K)^{\text {alg }}$ action factors through $\operatorname{Gal}(\bar{K} / K) \times_{\mathrm{Gal}\left(\bar{k} / k^{\prime}\right)} \operatorname{Gal}\left(\bar{k} / k^{\prime}\right)^{\text {alg }}$. In fact, Proposition 6.6 implies that the action factors through $\operatorname{Gal}(\bar{K} / K) \times_{\operatorname{Gal}\left(\bar{k} / k^{\prime}\right)} M_{q^{\prime}}$, where $q^{\prime}=\left|k^{\prime}\right|$, so the morphism $\mathbb{G}_{m} \rightarrow M_{q^{\prime}}^{0}=\operatorname{ker}\left(M_{q^{\prime}} \rightarrow \operatorname{Gal}\left(\bar{k} / k^{\prime}\right)\right)$ provides the weight decomposition. This is compatible with the Galois action since $M_{q^{\prime}}$ is commutative (being a quotient of $\left.\mathbb{Z}^{\mathrm{alg}}\right)$, so $\mathbb{G}_{m}$ lies in the centre of $\operatorname{Gal}(\bar{K} / K) \times_{\mathrm{Gal}\left(\bar{k} / k^{\prime}\right)} M_{q^{\prime}}$.

We may now adapt Corollary 6.15 to see that this is quasiformal, noting that all of the quasi-isomorphisms above extend naturally to the filtered algebras of Corollary 6.15.

Corollary 7.4 Let $X$ and $R$ be as above. Then the homotopy groups $\varpi_{n}^{\text {ét }}\left(X_{\bar{K}}\right)$ are potentially unramified and mixed as Galois representations, giving them a canonical weight decomposition. They may also be recovered from the Leray spectral sequence, as in Corollary 6.16. 
Corollary 7.5 If $L$ is a set of primes including $\ell$, and

(1) $\pi_{f}^{\text {et }}(X)^{\wedge} L$ is $(N+1)$-good relative to ${ }^{\mathrm{pnr}} \varpi_{f}^{t}\left(X_{\bar{K}}^{\wedge^{L}}\right)$,

(2) $\pi_{n}^{\text {ét }}\left(X^{\wedge_{L}}\right) \otimes_{\widehat{\mathbb{Z}}} \mathbb{Q}_{\ell}$ is finite-dimensional for all $1<n \leq N$, and

(3) the action of $\operatorname{ker}\left(\pi_{f}^{\text {ét }}\left(X_{\bar{K}}\right)^{\wedge L} \rightarrow \pi_{f}^{t}\left(X_{\bar{V}}\right)^{\wedge L}\right)$ on $\pi_{n}^{\text {ét }}\left(X_{\bar{K}}^{\wedge^{L}}\right) \otimes_{\widehat{\mathbb{Z}}} \mathbb{Q}_{\ell}$ is unipotent for all $1<n \leq N$,

then the Galois action on $\pi_{n}^{\text {ét }}\left(X_{\bar{K}}^{\wedge L}\right) \otimes_{\widehat{\mathbb{Z}}} \mathbb{Q}_{\ell}$ is potentially unramified and mixed, giving it a canonical weight decomposition. It may also be recovered from the Leray spectral sequence.

Proof Substitute $R=\pi_{f}^{t}\left(X_{\bar{V}}\right)^{L, \text { red }}$ into Corollaries 7.4 and 6.16 and Theorem 3.40.

Note that if $L$ does not contain $p$, then the third condition of the Corollary is vacuous.

\subsection{Potentially good reduction, $\ell=p$}

7.2.1 Convergent isocrystals Let $X, \bar{X}, V^{\prime}, K, K^{\prime}, k^{\prime}$ etc. be as in the previous section, but with $\ell=p$. Let $W^{\prime}=W\left(k^{\prime}\right)$, the ring of Witt vectors over $k^{\prime}$, and $K_{0}^{\prime}$ the fraction field of $W^{\prime}$; let $W^{\mathrm{nr}}:=W(\bar{k})$, with $K_{0}^{\mathrm{nr}}$ its fraction field. Choose a homomorphism $\sigma: K^{\prime} \rightarrow K^{\prime}$ extending the natural action of the Frobenius operator $\phi$ on $W\left(k^{\prime}\right) \subset K^{\prime}$. Assume moreover that $T_{V^{\prime}}=D_{V^{\prime}}$, a normal crossings divisor, or more generally that $D_{V^{\prime}}$ corresponds to a $\log$ structure.

Definition 7.6 Let $\mathrm{MF}_{\left(\bar{X}_{V^{\prime}}, D_{V^{\prime}}\right) / K^{\prime}}^{\nabla}$ be the category of filtered convergent $F$-isocrystals on $\left(\bar{X}_{V^{\prime}}, D_{V^{\prime}}\right)$, as in [50, Section 1] (or [33, 6.9] when $K^{\prime}$ is unramified, noting that the construction extends to ramified rings, as mentioned at the end of $[33,1.14]$ ).

Roughly speaking, an object of $\operatorname{MF}_{\left(\bar{X}_{V^{\prime}}, D_{V^{\prime}}\right) / K^{\prime}}^{\nabla^{\prime}}$ consists of an $F$-isocrystal $\left(E, \phi_{E}\right)$ on $\left(\bar{X}_{k}, D_{k}\right) / W$, together with a filtration Fil $i_{\mathscr{E}} \mathscr{E}$ of $\mathscr{E}$ satisfying Griffiths transversality with respect to $\nabla_{\mathscr{E}}$, where $\left(\mathscr{E}, \nabla_{\mathscr{E}}\right)$ is the module with logarithmic connection on $\left(\bar{X}_{K^{\prime}}, D_{K^{\prime}}\right)$ obtained by base change from the evaluation of $E$ on the $p$-adic completion of $\left(\bar{X}_{V^{\prime}}, D_{V^{\prime}}\right)$.

7.2.2 Crystalline étale sheaves We now introduce crystalline étale sheaves, as in Faltings [8, V(f)] or Andreatta and Iovita [1]. 
Definition 7.7 We define the category of associations on $\left(\bar{X}_{V^{\prime}}, D_{V^{\prime}}\right)$ to consist of triples $(\mathbb{V}, \iota, E)$, where

(1) $\mathbb{V}$ is a smooth $\mathbb{Q}_{p}$-sheaf on $X_{K^{\prime}}$,

(2) $E \in \mathrm{MF}_{\left(\bar{X}_{V^{\prime}}, D_{V^{\prime}}\right)}^{\nabla}(\Phi)$,

(3) $\iota$ is an association isomorphism [33, Section 6.13], ie a collection of isomorphisms

$$
\iota_{U}: \mathbb{V} \otimes_{\mathbb{Q}_{p}} B_{\text {cris }}(\hat{U}) \rightarrow E\left(B_{\text {cris }}(\hat{U})\right)
$$

for $U \rightarrow X_{V^{\prime}}$ étale, compatible with the filtrations and semilinear Frobenius automorphisms, and with morphisms over $X$, so that $\iota$ becomes an isomorphism of étale presheaves. Here, $B_{\text {cris }}(\hat{U})$ is formed by applying Fontaine's construction to the $p$-adic completion $\widehat{U}$ of $U$.

A morphism $f:(\mathbb{V}, \iota, E) \rightarrow\left(\mathbb{V}^{\prime}, \iota^{\prime}, E^{\prime}\right)$ in the category of associations consists of a morphism $f^{\text {ét: }} \mathbb{V} \rightarrow \mathbb{V}^{\prime}$ and a morphism $f^{\text {cris }}: E \rightarrow E^{\prime}$ such that $f^{\text {cris }} \circ \iota=$ $\iota^{\prime} \circ f^{\text {ét: }} \mathbb{V} \otimes_{\mathbb{Q}_{p}} B_{\text {cris }}(\hat{U}) \rightarrow E^{\prime}\left(B_{\text {cris }}(\widehat{U})\right)$ for all $U$.

The following lemma is a counterpart to [8, Lemma 5.5], which gives the corresponding statements for the forgetful functor from associations to $\mathrm{MF}_{\left(X_{V^{\prime}}, D_{V^{\prime}}\right)}^{\nabla}$.

Proposition 7.8 The forgetful functor $(\mathbb{V}, \iota, E) \mapsto \mathbb{V}$ from the category of associations to the category of smooth $\mathbb{Q}_{p}$-sheaves on $X_{K^{\prime}}$ is full and faithful. Its essential image is stable under extensions and subquotients.

Proof Given associations $(\mathbb{V}, \iota, E),\left(\mathbb{V}^{\prime}, \iota^{\prime}, E^{\prime}\right)$, note $\left(\mathbb{V}^{\vee} \otimes \mathbb{V},\left(\iota^{\vee}\right)^{-1} \otimes \iota^{\prime}, E^{\vee} \otimes E^{\prime}\right)$ is another association. Giving a morphism $f^{\text {ét: }} \mathbb{V} \rightarrow \mathbb{V}^{\prime}$ amounts to giving an element of $\mathrm{H}^{0}\left(X_{K}, \mathbb{V}^{\vee} \otimes \mathbb{V}^{\prime}\right)$, or equivalently a Galois-invariant element of $\mathrm{H}^{0}\left(X_{\bar{K}}, \mathbb{V}^{\vee} \otimes \mathbb{V}^{\prime}\right)$. By $[8,5.6]$, the map

$$
\left(\iota^{\vee}\right)^{-1} \otimes \iota^{\prime}: \mathrm{H}^{*}\left(X_{\bar{K}}, \mathbb{V}^{\vee} \otimes \mathbb{V}^{\prime}\right) \otimes_{\mathbb{Q}_{p}} B_{\text {cris }} \rightarrow \mathrm{H}_{\text {cris }}^{*}\left(X_{k} / W, E^{\vee} \otimes E^{\prime}\right) \otimes_{K^{\prime}{ }_{0}} B_{\text {cris }}
$$

is an isomorphism. Taking Galois-invariant and Frobenius-invariant elements in $\mathrm{Fil}^{0}$, this gives an isomorphism

$$
\left(\iota^{\vee}\right)^{-1} \otimes \iota^{\prime}: \mathrm{H}^{0}\left(X_{\bar{K}}, \mathbb{V}^{\vee} \otimes \mathbb{V}^{\prime}\right)^{\mathrm{Gal}\left(\bar{K} / K^{\prime}\right)} \rightarrow \mathrm{Fil}^{0} \mathrm{H}_{\text {cris }}^{0}\left(X_{k} / W, E^{\vee} \otimes E^{\prime}\right)^{\phi},
$$

so there is a unique Frobenius-equivariant morphism $f^{\text {cris }}: E \rightarrow E^{\prime}$ preserving the Hodge filtration such that the diagrams

$$
\begin{gathered}
\mathbb{V} \otimes_{\mathbb{Q}_{p}} B_{\text {cris }}(\hat{U}) \stackrel{\iota}{\longrightarrow} E\left(B_{\text {cris }}(\widehat{U})\right) \\
f^{\text {ét } \otimes_{\mathbb{Q}_{p}} B_{\text {cris }} \downarrow} \underset{f^{\text {cris }}\left(B_{\text {cris }}(\widehat{U})\right)}{ } \\
\mathbb{V}^{\prime} \otimes_{\mathbb{Q}_{p}} B_{\text {cris }}(\widehat{U}) \stackrel{\iota^{\prime}}{\longrightarrow} E^{\prime}\left(B_{\text {cris }}(\widehat{U})\right)
\end{gathered}
$$


commute. This shows that the forgetful functor is full and faithful.

To see that the essential image is stable under extensions, observe that extensions of $\mathbb{V}$ by $\mathbb{V}^{\prime}$ are parametrised by elements $a$ of $\mathrm{H}^{1}\left(X_{K^{\prime}}, \mathbb{V}^{\vee} \otimes \mathbb{V}^{\prime}\right)$. The isomorphisms above then show that $\left(\left(\iota^{\vee}\right)^{-1} \otimes \iota^{\prime}\right)(a)$ is a Frobenius-equivariant element of Fil $^{0} \mathrm{H}_{\text {cris }}^{1}\left(X_{k} / W, E^{\vee} \otimes E^{\prime}\right)$, so gives a unique extension of $(\mathbb{V}, \iota, E)$ by $\left(\mathbb{V}^{\prime}, \iota^{\prime}, E^{\prime}\right)$ in the category of associations.

Finally, note that the subquotient of an extension is an extension of subquotients, so it suffices to show that the essential image contains subquotients of semisimple objects. Since such a subquotient $\mathbb{V}^{\prime}$ of $\mathbb{V}$ is isomorphic to a direct summand, we have an idempotent endomorphism $\pi$ of $\mathbb{V}$ with $\operatorname{ker} \pi \cong \mathbb{V}^{\prime}$. Since the forgetful functor is full, $\pi$ lifts to an idempotent endomorphism $\tilde{\pi}$ of $(\mathbb{V}, \iota, E)$, so $\mathbb{V}^{\prime}$ underlies ker $\tilde{\pi}$.

Definition 7.9 Say that a smooth $\mathbb{Q}_{p}$-sheaf $\mathbb{V}$ on $X_{K^{\prime}}$ is crystalline if it lies in the essential image of the forgetful functor from the category of associations.

Proposition 7.10 The fibre functors $(\mathbb{V}, \iota, E) \mapsto \mathbb{V}_{\bar{x}}$ make the category of associations into a multifibred Tannakian category. The corresponding pro-algebraic groupoid $\varpi_{f}^{\text {ét }}\left(X_{K^{\prime}}\right)^{\text {cris }}$ is a quotient of $\varpi_{f}^{\text {ét }}\left(X_{K^{\prime}}\right)$. Moreover, $\varpi_{f}^{\text {ét }}\left(X_{K^{\prime}}\right)^{\text {cris }}$ is the Malcev completion of $\pi_{f}^{\text {ét }}\left(X_{K^{\prime}}\right)$ with respect to the reductive quotient $\varpi_{f}^{\text {ét }}\left(X_{K^{\prime}}\right)^{\text {cris,red }}$.

Proof Associations form a $\mathbb{Q}_{p}$-linear rigid abelian tensor category, with $(\mathbb{V}, \iota, E) \otimes$ $\left(\mathbb{V}^{\prime}, \iota^{\prime}, E^{\prime}\right)=\left(\mathbb{V} \otimes_{\mathbb{Q}_{p}} \mathbb{V}^{\prime}, \iota \otimes \iota^{\prime}, E \otimes_{\mathcal{O}_{X_{k}}, \text { cris }} E^{\prime}\right)$ and $(\mathbb{V}, \iota, E)^{\vee}=\left(\mathbb{V}^{\vee},\left(\iota^{-1}\right)^{\vee}, E^{\vee}\right)$.

By Proposition 7.8, associations are equivalent to the Tannakian subcategory of crystalline étale sheaves in $\operatorname{Rep}\left(\varpi_{f}^{\text {ét }}\left(X_{K^{\prime}}\right)\right)$. Thus the forgetful functor from associations to smooth $\mathbb{Q}_{p}$-sheaves corresponds to a surjection $\varpi_{f}^{\text {ét }}\left(X_{K^{\prime}}\right) \rightarrow \varpi_{f}^{\text {ét }}\left(X_{K^{\prime}}\right)^{\text {cris }}$ of proalgebraic groupoids (with the same object set).

For $\rho: \pi_{f}^{\text {ét }}\left(X_{K^{\prime}}\right) \rightarrow \varpi_{f}^{\text {ét }}\left(X_{K^{\prime}}\right)^{\text {cris,red }}$, representations of $\varpi_{f}^{\text {ét }}\left(X_{K^{\prime}}\right)^{\rho, \text { Mal }}$ are smooth $\mathbb{Q}_{p}$-sheaves on $X_{K^{\prime}}$ which are Artinian extensions of semisimple crystalline étale sheaves. By Proposition 7.8, this is equivalent to the category $\operatorname{Rep}\left(\varpi_{f}^{\text {ét }}\left(X_{K^{\prime}}\right)^{\text {cris }}\right)$ of associations.

Definition 7.11 Say that a smooth $\mathbb{Q}_{p}$-sheaf $\mathbb{V}$ on $X_{K}$ is potentially crystalline if $\left.\mathbb{V}\right|_{X_{K^{\prime \prime}}}$ is crystalline for some finite extension $K^{\prime} \subset K^{\prime \prime}$.

\subsubsection{Equivariant pro-algebraic fundamental groups}

Definition 7.12 Define ${ }^{\text {cris, } \mathrm{K}^{\prime}} \varpi_{f}^{\text {ét }}\left(X_{\bar{K}}\right)$ to be the image of $\varpi_{f}^{\text {ét }}\left(X_{\bar{K}}\right) \rightarrow \varpi_{f}^{\text {ét }}\left(X_{K^{\prime}}\right)^{\text {cris }}$. 
Note that we can also characterise ${ }^{\text {cris, } \mathrm{K}^{\prime}} \varpi_{f}^{\text {et }}\left(X_{\bar{K}}\right)$ as

$$
\operatorname{ker}\left(\varpi_{f}^{\mathrm{e} t}\left(X_{K^{\prime}}\right)^{\mathrm{cris}} \rightarrow \operatorname{Gal}\left(\bar{K} / K^{\prime}\right)^{\mathrm{cris}}=\varpi_{f}^{\mathrm{e} t}\left(\operatorname{Spec} K^{\prime}\right)^{\mathrm{cris}}\right),
$$

using the right-exactness of pro-algebraic completion. Thus

$$
\varpi_{f}^{\text {ét }}\left(X_{K^{\prime}}\right)^{\text {cris }}={ }^{\text {cris, } \mathrm{K}^{\prime}} \varpi_{f}^{\text {ét }}\left(X_{\bar{K}}\right) \rtimes \operatorname{Gal}\left(\bar{K} / K^{\prime}\right)^{\text {cris }},
$$

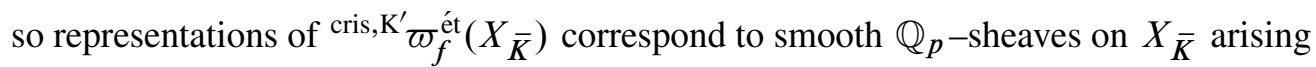
as subsheaves of pullbacks of crystalline étale $\mathbb{Q}_{p}$-sheaves on $X_{K^{\prime}}$.

\section{Definition 7.13 Define}

$$
{ }^{\text {pcris }} \varpi_{f}^{\text {ét }}\left(X_{\bar{K}}\right):={\overleftarrow{K^{\prime \prime}}}^{\text {cris, } \mathrm{K}^{\prime \prime}} \varpi_{f}^{\text {ét }}\left(X_{\bar{K}}\right)
$$

where the limit is taken over all finite Galois extensions $K^{\prime} \subset K^{\prime \prime}$.

Finite-dimensional representations of ${ }^{\text {pcris }} \varpi_{f}^{\text {ét }}\left(X_{\bar{K}}\right)$ thus correspond to smooth $\mathbb{Q}_{p}-$ sheaves on $X_{\bar{K}}$ arising as subsheaves of pullbacks of potentially crystalline smooth $\mathbb{Q}_{p}$-sheaves on $X_{K}$.

Since $\mathcal{G}=\lim _{K^{\prime \prime}}\left(\operatorname{Gal}\left(\bar{K} / K^{\prime \prime}\right)^{\text {cris }} \times_{\operatorname{Gal}\left(\bar{K} / K^{\prime \prime}\right)} \operatorname{Gal}(\bar{K} / K)\right)$, this gives an isomorphism

$$
\lim _{K^{\prime \prime}}\left(\operatorname{Gal}(\bar{K} / K) \times_{\operatorname{Gal}\left(\bar{K} / K^{\prime \prime}\right)} \varpi_{f}^{\text {ét }}\left(X_{K^{\prime \prime}}\right)^{\text {cris }}\right) \cong{ }^{\text {pcris }} \varpi_{f}^{\text {ét }}\left(X_{\bar{K}}\right) \rtimes \mathcal{G}^{\text {pcris }}
$$

so the Galois action on ${ }^{\text {pcris }} \varpi_{f}^{\text {et }}\left(X_{\bar{K}}\right)$ is algebraic and potentially crystalline.

Lemma 7.14 The map $\varpi_{f}^{\text {ét }}\left(X_{\bar{K}}\right) \rightarrow{ }^{\mathrm{pnr}} \varpi_{f}\left(\bar{X}_{\bar{K}}\right)$ factors through ${ }^{\mathrm{pcris}} \varpi_{f}^{\text {ét }}\left(X_{\bar{K}}\right)$.

Proof Since

$$
\operatorname{Gal}(\bar{K} / K)^{\mathrm{pnr}} \ltimes^{\mathrm{pnr}} \varpi_{f}\left(\bar{X}_{\bar{K}}\right)={\overleftarrow{K^{\prime \prime}}}_{\lim } \operatorname{Gal}(\bar{K} / K) \times_{\mathrm{Gal}\left(\bar{k} / k^{\prime \prime}\right)} \varpi_{f}\left(\bar{X}_{k^{\prime \prime}}\right)
$$

it suffices to show that the map $\varpi_{f}\left(X_{K^{\prime \prime}}\right) \rightarrow \varpi_{f}\left(\bar{X}_{k^{\prime \prime}}\right)$ factors through $\varpi_{f}^{\text {ét }}\left(X_{K^{\prime \prime}}\right)^{\text {cris }}$. By looking at representations, this is equivalent to saying that every smooth $\mathbb{Q}_{p}$-sheaf on $\bar{X}_{k^{\prime \prime}}$ pulls back to give a crystalline étale sheaf on $X_{V^{\prime \prime}}$. This now follows from [21, 4.1.1], which shows that smooth $\mathbb{Q}_{p}$-sheaves on $\bar{X}_{k^{\prime \prime}}$ correspond to unit-root $F$-lattices on $X_{V^{\prime \prime}}$.

Definition 7.15 Any field extension $K^{\prime} \rightarrow K^{\prime \prime}$ gives a pullback functor

$$
\operatorname{MF}_{\left(\bar{X}_{V^{\prime}}, D_{V^{\prime}}\right) / K^{\prime}}^{\nabla} \rightarrow \operatorname{MF}_{\left(\bar{X}_{V^{\prime \prime}}, D_{V^{\prime \prime}}\right) / K^{\prime \prime}}^{\nabla},
$$


and we set

$$
\operatorname{MF}_{\left(\bar{X}_{\bar{V}}, D_{\bar{V}}\right) / \bar{K}}^{\nabla}:=\underset{K^{\prime \prime}}{\lim } \operatorname{MF}_{\left(\bar{X}_{V^{\prime \prime}}, D_{V^{\prime \prime}}\right) / K^{\prime \prime}}^{\nabla},
$$

where $K^{\prime \prime}$ ranges over all finite field extensions $K^{\prime} \subset K^{\prime \prime}$.

Representations of $\operatorname{Gal}(\bar{K} / K)^{\text {pcris, } 0} \ltimes{ }^{\text {pcris }} \varpi_{f}^{\text {ét }}\left(X_{\bar{K}}\right)$ are just representations of

$$
\overleftarrow{K}_{K^{\prime \prime}} \varpi_{f}^{\text {ét }}\left(X_{K^{\prime \prime}}\right)^{\text {cris }}
$$

so the category of finite-dimensional representations is

$$
\underset{K^{\prime \prime}}{\lim } \operatorname{FD} \operatorname{Rep}\left(\varpi_{f}^{\text {ét }}\left(X_{K^{\prime \prime}}\right)^{\text {cris }}\right) \text {. }
$$

Definition 7.16 Making use of the forgetful functor from associations to filtered convergent $F$-isocrystals, the observation above gives us a $\mathbb{Q}_{p}$-linear functor

$$
D_{\text {pcris }}^{X}: \operatorname{FD~Rep}\left(\operatorname{Gal}(\bar{K} / K)^{\text {pcris }, 0} \ltimes{ }^{\text {pcris }} \varpi_{f}^{\text {ét }}\left(X_{\bar{K}}\right)\right) \rightarrow \operatorname{MF}_{\left(\bar{X}_{\bar{V}}, D_{\bar{V}}\right) / \bar{K}}^{\nabla_{\bar{K}}}
$$

Say that an object of $\operatorname{MF}_{\left(\bar{X}_{\bar{V}}, D_{\bar{V}}\right) / \bar{K}}$ is potentially admissible if it lies in the essential image of $D_{\text {pcris }}^{X}$.

Note that $D_{\text {pcris }}^{\text {Spec } K}=D_{\text {pcris }}$.

Definition 7.17 Given a $\mathcal{G}^{0}$-equivariant affine scheme $Y$ over $\mathbb{Q}_{p}$, define the affine scheme $D_{\text {pcris }}(Y)$ over $K_{0}^{\mathrm{nr}}$ by

$$
D_{\text {pcris }}(Y)=\operatorname{Spec} D_{\text {pcris }} O(Y) .
$$

Observe that $O(Y)$ is therefore an ind-object of (ie a sum of objects in) the category $\operatorname{MF}_{(\operatorname{Spec} \bar{V}, \varnothing) / \bar{K}}^{\nabla}$

Proposition 7.18 The category of finite-dimensional $D_{\text {pcris }}\left({ }^{\text {pcris }} \varpi_{f}^{\text {ét }}\left(X_{\bar{K}}\right)\right)$-representations in potentially admissible objects of $\mathrm{MF}_{(\operatorname{Spec} \bar{V}, \varnothing) / \bar{K}}^{\nabla}$ is equivalent to the category of finite-dimensional $\mathcal{G}^{\text {pcris, } 0} \ltimes{ }^{\text {pcris }} \varpi_{f}^{\text {ét }}\left(X_{\bar{K}}\right)$-representations, which in turn is equivalent to the category of potentially admissible objects of $\mathrm{MF}_{\left(\bar{X}_{\bar{V}}, D_{\bar{V}}\right) / \bar{K}}^{\nabla}$.

For any point $x \in X_{\bar{V}}(\bar{K})$, the associated fibre functor from $D_{\text {pcris }}\left({ }^{\text {pcris }} \varpi_{f}^{\text {et }}\left(X_{\bar{K}}\right)\right)-$ representations to $\mathrm{MF}_{(\operatorname{Spec} \bar{V}, \varnothing) / \bar{K}}^{\nabla}$ corresponds under this equivalence to the pullback

$$
x^{*}: \operatorname{MF}_{\left(\bar{X}_{\bar{V}}, D_{\bar{V}}\right) / \bar{K}}^{\nabla} \rightarrow \operatorname{MF}_{(\operatorname{Spec} \bar{V}, \varnothing) / \bar{K}}^{\nabla} .
$$


Proof A $D_{\text {pcris }}\left({ }^{\text {pcris }} \varpi_{f}^{\text {ét }}\left(X_{\bar{K}}\right)\right)$-representation $V$ in potentially admissible objects of $\left.\operatorname{MF}_{(\operatorname{Spec}}^{\nabla}, \varnothing\right) / \bar{K}$ consists of potentially admissible objects $V(x) \in \operatorname{MF}_{(\operatorname{Spec} \bar{V}, \varnothing) / \bar{K}}^{\nabla}$ for all $x \in \mathrm{Ob}\left({ }^{\text {prris }} \varpi_{f}^{\text {et }}\left(X_{\bar{K}}\right)\right)$, together with coassociative morphisms

$$
V(y) \rightarrow V(x) \otimes D_{\text {pcris }} O\left({ }^{\text {pcris }} \varpi_{f}^{\text {ét }}\left(X_{\bar{K}}\right)(x, y)\right)
$$

in $\mathrm{MF}_{(\operatorname{Spec} \bar{V}, \varnothing) / \bar{K}}^{\nabla}$.

Since $D_{\text {pcris }}$ gives an equivalence between $\mathcal{G}^{\text {pcris, } 0}$-representations and potentially admissible objects of $\operatorname{MF}_{(\operatorname{Spec} \bar{V}, \varnothing) / \bar{K}}^{\nabla}$, the description above shows that it defines the required equivalence from $\mathcal{G}^{\text {pcris, } 0} \ltimes{ }^{\text {pcris }} \varpi_{f}^{\text {et }}\left(X_{\bar{K}}\right)$-representations.

Now, $\mathcal{G}^{\text {pcris, } 0} \ltimes^{\text {pcris }} \varpi_{f}^{\text {et }}\left(X_{\bar{K}}\right) \cong \lim _{K^{\prime \prime}} \varpi_{f}^{\text {ét }}\left(\bar{X}_{K^{\prime \prime}}\right)^{\text {cris }}$, so we may apply the functor $D_{\text {pcris }}^{X}$ from Definition 7.16, mapping to potentially admissible objects in $\operatorname{MF}_{\left(\bar{X}_{\bar{V}}, D_{\bar{V}}\right) / \bar{K}}$. By [8, Lemma 5.5], this functor is full and faithful, so gives us the second equivalence required.

Definition 7.19 Define

$$
\operatorname{Isoc}\left(\left(\bar{X}_{\bar{k}}, D_{\bar{k}}\right) / K_{0}^{\mathrm{nr}}\right):=\underset{K^{\prime \prime}}{\lim } \operatorname{Isoc}\left(\left(\bar{X}_{k^{\prime \prime}}, D_{k^{\prime \prime}}\right) / K^{\prime \prime}\right)
$$

to be the category of isocrystals on $\lim _{\leftarrow}\left(\bar{X}_{k^{\prime \prime}}, D_{k^{\prime \prime}}\right) / K^{\prime \prime}$, where the limit is taken over finite extensions $K^{\prime} \subset K^{\prime \prime}$.

Proposition 7.20 The category of finite-dimensional $D_{\text {pcris }}\left({ }^{\text {pcris }} \varpi_{f}^{\text {ét }}\left(X_{\bar{K}}\right)\right)$-representations over $K_{0}^{\mathrm{nr}}$ is equivalent to a full subcategory of $\operatorname{Isoc}\left(\left(\bar{X}_{\bar{k}}, D_{\bar{k}}\right) / K_{0}^{\mathrm{nr}}\right)$. This subcategory is the smallest full abelian subcategory containing the potentially admissible objects of $\mathrm{MF}_{\left(\bar{X}_{\bar{V}}, D_{\bar{V}}\right) / \bar{K}}$.

Proof Write $G:={ }^{\text {pcris }} \varpi_{f}^{\text {et }}\left(X_{\bar{K}}\right)$, and let $\mathbb{O}(G)$ be the universal $G$-representation in smooth $\mathbb{Q}_{p}$-sheaves on $X_{\bar{K}}$, as defined in Definition 2.75. Following through the proof of Proposition 7.18, the functor from $D_{\text {pcris }}(G)$-representations in potentially admissible objects of $\mathrm{MF}_{(\operatorname{Spec} \bar{V}, \varnothing) / \bar{K}}^{\nabla}$ to $\operatorname{MF}_{\left(\bar{X}_{\bar{V}}, D_{\bar{V}}\right) / \bar{K}}^{\nabla}$ is given by

$$
F(A):=A \otimes D_{\text {pcris }}(G) D_{\text {pcris }}^{X} \mathbb{O}(G),
$$

while its inverse is

$$
F_{*}(\mathscr{A}):=\underset{K^{\prime \prime}}{\lim } \mathrm{H}_{\text {cris }}^{0}\left(\left(\bar{X}_{k^{\prime \prime}}, D_{k^{\prime \prime}}\right), \mathscr{A} \otimes D_{\text {pcris }}^{X} \mathbb{O}(G)\right) .
$$


The same formulae define left exact functors $F, F_{*}$ between the category of finitedimensional $D_{\text {pcris }}(G)$-representations and $\operatorname{Isoc}\left(\left(\bar{X}_{\bar{k}}, D_{\bar{k}}\right) / K_{0}^{\mathrm{nr}}\right)$. For any point $x \in$ $X(\bar{K})$,

$$
F(A)_{x}=A \otimes^{D_{\text {pris }}(G)} D_{\text {pcris }}\left(\mathbb{O}(G)_{x}\right)=A \otimes^{D_{\text {pcris }}(G)} D_{\text {pcris }}(O(G)(x,-))=A(x),
$$

so $F$ is exact.

For any $D_{\text {pcris }}(G)$-representation $A$,

$$
\begin{aligned}
& F_{*} F(A)=A \otimes D_{\text {pcris }}(G) \underset{K^{\prime \prime}}{\lim } H_{\text {cris }}^{0}\left(\left(\bar{X}_{k^{\prime \prime}}, D_{k^{\prime \prime}}\right), D_{\text {pcris }}^{X} \mathbb{O}(G) \otimes D_{\text {pcris }}^{X} \mathbb{O}(G)\right) \\
& =A \otimes{ }^{D_{\text {pris }}(G)} D_{\text {peris }} O(G) \\
& =A \text {. }
\end{aligned}
$$

Moreover, $F_{*}$ is right adjoint to $F$, since a morphism $A \rightarrow F_{*}\left(\mathscr{A}^{\prime}\right)$ is equivalent to a $G$-equivariant morphism $A \otimes \mathcal{O}_{X \text {,cris }} \rightarrow \mathscr{A}^{\prime} \otimes D_{\text {pcris }}^{X} \mathbb{O}(G)$ of isocrystals, which is equivalent to a $G$-equivariant $D_{\text {pcris }}^{X} \mathbb{O}(G)$-linear morphism $A \otimes D_{\text {pcris }}^{X} \mathbb{O}(G) \rightarrow$ $\mathscr{A}^{\prime} \otimes D_{\text {pcris }}^{X} \mathbb{O}(G)$, which (taking $G$-invariants) is just a morphism $F(A) \rightarrow \mathscr{A}^{\prime}$. These two statements combine to show that $F$ is full and faithful.

Since $F$ is exact, its essential image is an abelian subcategory. Proposition 7.18 ensures that it contains all potentially admissible objects of $\operatorname{MF}_{\left(\bar{X}_{\bar{V}}, D_{\bar{V}}\right) / \bar{K}}^{\nabla}$, so we need only show that anything in the image of $F$ is in the abelian subcategory generated by these potentially admissible objects.

Given any $D_{\text {pcris }}(G)$-representation $A$, there exists a canonical embedding $A \hookrightarrow$ $A \otimes D_{\text {pcris }}(O(G))$, which is a sum of objects of $\left.\operatorname{MF}_{(\operatorname{Spec}}^{\nabla}, \varnothing\right) / \bar{K}$. Thus for some finite-dimensional subobject $U$, we have an embedding $A \hookrightarrow U$. Replacing $A$ with $U / A$, we get an embedding $U / A \hookrightarrow U^{\prime}$, so $A=\operatorname{ker}\left(U \rightarrow U^{\prime}\right)$, and hence $F(A)=$ $\operatorname{ker}\left(F(U) \rightarrow F\left(U^{\prime}\right)\right)$. Since $F(U)$ and $F\left(U^{\prime}\right)$ are potentially admissible objects of $\operatorname{MF}_{\left(\bar{X}_{\bar{V}}, D_{\bar{V}}\right) / \bar{K}}^{\nabla_{\bar{K}}}$, this completes the proof.

7.2.4 Crystalline homotopy types Fix a Galois-equivariant quotient $R$ of ${ }^{\text {pcris }} \varpi_{f}^{\text {ét }}\left(X_{\bar{K}}\right)^{\text {red }}$, or rather of its full subgroupoid on objects $X(\bar{K})$.

Definition 7.21 Let $\mathscr{F} \rightarrow \mathscr{C}_{\text {cris }}^{\bullet}(\mathscr{F})$ be a choice of functor from isocrystals to cosimplicial sheaves on the log-crystalline site, with the property that $\mathscr{C}_{\text {cris }}^{\bullet}(\mathscr{F})$ is a resolution of $\mathscr{F}$, compatible with tensor products, and acyclic for log-crystalline cohomology. Examples of such a functor are given in [35, page 17], or by denormalising the construction $D R$ of $[33,4.29 .2]$. In both cases, the resolution is given by first choosing 
a resolution which is acyclic for the derived functor between crystalline and Zariski sites (such as denormalisation of the de Rham complex), then taking a Čech resolution.

Define

$$
\mathrm{C}_{\text {cris }}^{\bullet}(Y, \mathscr{F}):=\Gamma\left(Y, \mathscr{C}_{\text {cris }}^{\bullet}(\mathscr{F})\right),
$$

observing that this construction will also be compatible with tensor products.

Definition 7.22 Define the relative crystalline homotopy type $X_{\bar{k} \text {,cris }}^{D_{\text {pcis }}(R) \text {,Mal }}$ over $D_{\text {pcris }} R$ to be the pro-algebraic homotopy type in $\operatorname{Ho}\left(s \mathcal{E}\left(D_{\text {pcris }} R\right)_{*}\right)$ (over $\left.K_{0}^{\mathrm{nr}}\right)$ corresponding under Theorem 2.74 to the $D_{\text {pcris }}(R)$-representation

$$
\mathrm{C}_{\text {cris }}^{\bullet}\left(\left(\bar{X}_{\bar{k}}, D_{\bar{k}}\right), D_{\text {pcris }}^{X} \mathbb{O}(R)\right)
$$

in cosimplicial $K_{0}^{\mathrm{nr}}$-algebras, equipped with its natural augmentations to

$$
D_{\text {pcris }} O(R)(x,-)=\mathrm{C}_{\text {cris }}^{\bullet}\left(\operatorname{Spec} K_{0}^{\mathrm{nr}}, x^{*} D_{\text {pcris }}^{X} \mathbb{O}(R)\right)
$$

coming from elements $x \in X(\bar{V})$.

Lemma 7.23 There is a canonical equivalence between representations of

$$
\varpi_{f}\left(X_{\bar{k}} / K_{0}^{\mathrm{nr}}\right)_{\text {cris }}^{D_{\text {pris }}(R), \text { Mal }}
$$

and a full subcategory of $\operatorname{Isoc}\left(\left(\bar{X}_{\bar{k}}, D_{\bar{k}}\right) / K_{0}^{\mathrm{nr}}\right)$. Objects of this category are Artinian extensions of those isocrystals corresponding under Proposition 7.20 to $D_{\text {pcris }}(R)-$ representations.

Proof This is [32, Theorem 2.28]. An alternative approach would be to note that the proof of [35, Theorem 2.9] carries over to nonnilpotent torsors.

Definition 7.24 For a topos $\mathcal{T}$, if $\mathscr{C}_{\mathcal{T}}^{\bullet}(\mathscr{S})$ is a canonical cosimplicial $\mathcal{T}$-resolution of a sheaf $\mathscr{S}$ of algebras on $X$, with $\mathrm{C}_{\mathcal{T}}^{\bullet}(X, \mathscr{Y}):=\Gamma\left(X, \mathscr{C}_{\mathcal{T}}^{\bullet}(\mathscr{Y})\right)$, then for any morphism $f: X \rightarrow Y$ we have a bicosimplicial algebra $\mathrm{C}_{\mathcal{T}}^{\bullet}\left(Y, f_{*} \mathscr{C}_{\mathcal{T}}^{\bullet}(\mathscr{S})\right)$, and we define

$$
\mathrm{C}_{\mathcal{T}}^{\bullet}(f, \mathscr{S}):=\tau^{\prime \prime} \mathrm{C}_{\mathcal{T}}^{\bullet}\left(Y, f_{*} \mathscr{C}_{\mathcal{T}}^{\bullet}(\mathscr{S})\right) \in F c \mathrm{Alg},
$$

defined as in Definition 4.33.

Definition 7.25 If we write $j$ for the embedding $X \hookrightarrow \bar{X}$, define the filtered relative crystalline homotopy type $\left(X_{\bar{k} \text {,cris }}, j_{\bar{k} \text {,cris }}\right)^{D_{\text {pcris }}(R) \text {,Mal }}$ over $D_{\text {pcris }} R$ to be the filtered pro-algebraic homotopy type in $\operatorname{Ho}\left(s \mathcal{E}\left(D_{\text {pcris }} R\right)_{*}\right)$ (over $\left.K_{0}^{\mathrm{nr}}\right)$ corresponding under Theorem 4.22 to the filtered $D_{\text {pcris }}(R)$-representation

$$
\mathrm{C}_{\text {cris }}^{\bullet}\left(j_{\bar{k}, \text { cris }}, D_{\text {pcris }}^{X} \mathbb{O}(R)\right)
$$


in cosimplicial $K_{0}^{\mathrm{nr}}$-algebras, equipped with its natural augmentations to

$$
D_{\text {pcris }} O(R)(x,-)=\mathrm{C}_{\text {cris }}^{\bullet}\left(\operatorname{Spec} K_{0}^{\mathrm{nr}}, x^{*} D_{\text {pcris }}^{X} \mathbb{O}(R)\right)
$$

coming from elements $x \in X(\bar{V})$.

7.2.5 Comparison of homotopy types From now on, let $B:=B_{\text {cris }}(V)$ and $\widetilde{B}:=$ $\widetilde{B}_{\text {cris }}(V)$, from Definition 5.18.

Proposition 7.26 For any Galois-equivariant quotient $R$ of ${ }^{\text {pcris }} \varpi_{f}^{\text {et }}\left(X_{\bar{K}}\right)^{\text {red }}$, there is a chain of $\left(\phi, \mathcal{G}^{0}\right)$-equivariant quasi-isomorphisms

$$
X_{\bar{K} \text {,ét }}^{R, \mathrm{Mal}} \otimes_{\mathbb{Q}_{p}} \widetilde{B} \sim X_{\bar{k} \text {,cris }}^{D_{\text {pcris }} R, \mathrm{Mal}} \otimes_{K_{0}^{\mathrm{nr}}} \widetilde{B}
$$

in $s \operatorname{Aff}_{\widetilde{B}}(R)_{*}$.

Proof This amounts to establishing a chain of quasi-isomorphisms

$$
\mathrm{C}_{\text {ét }}^{\bullet}\left(X_{\bar{K}}, \mathbb{O}(R)\right) \otimes_{\mathbb{Q}_{p}} \widetilde{B} \sim \mathrm{C}_{\text {cris }}^{\bullet}\left(X_{\bar{k}} / K_{0}^{\mathrm{nr}}, D_{\text {pcris }}^{X} \mathbb{O}(R)\right) \otimes_{K_{0}^{\mathrm{nr}}} \widetilde{B}
$$

in $c \operatorname{Alg}_{\widetilde{B}}(R)_{*}$

In the notation of $[33,4.29,5.21], \mathrm{C}_{\text {cris }}^{\bullet}\left(X_{k} / K_{0}^{\mathrm{nr}}, D_{\text {pcris }}^{X} \mathbb{O}(R)\right)$ and $\mathrm{C}_{\text {et }}^{\bullet}\left(X_{\bar{K}}, \mathbb{O}(R)\right)$ are quasi-isomorphic to the denormalisations of $\mathbf{R} \Gamma_{\text {cris }}\left(D_{\text {pcris }}^{X} \mathbb{O}(R)\right)$ and $G C(\mathbb{O}(R), X(\bar{K}))$, since denormalisation and Thom-Sullivan are quasi-inverse up to homotopy (as in Remarks 3.31).

Since the affine group schemes $R / \mathbb{Q}_{p}$ and $D_{\text {pcris }}(R) / K_{0}^{\mathrm{nr}}$ are associated by an isomorphism

$$
B \otimes_{\mathbb{Q}_{p}} O(R) \cong B \otimes_{K_{0}^{\mathrm{nr}}} D_{\text {pcris }} O(R),
$$

the required result is then ibid. 6.15.1, combined with the observation in ibid. Proposition 6.19 that pullback preserves associations, thus ensuring that these associations are compatible with the augmentation maps coming from basepoints.

The proof of ibid. 6.15 .1 proceeds by adapting the isomorphisms on cohomology groups from $[8,5.6]$ to quasi-isomorphisms of DG algebras. Since the latter proves that the cohomological isomorphisms respect cup products, an alternative approach would be to extend the isomorphisms to quasi-isomorphisms of the minimal $E_{\infty}$-algebras they underlie. Remark 2.54 would then imply that the corresponding objects in $d g \hat{\mathcal{N}}(R)$ are weakly equivalent. 
Remark 7.27 When $L$ is a crystalline étale sheaf on $X_{K}$ and $R$ is the Zariski closure of the image of $\pi_{1}^{\text {et }}\left(X_{\bar{K}}, \bar{x}\right) \rightarrow \operatorname{GL}\left(L_{\bar{x}}\right)$ with nilpotent monodromy around each component of the divisor, then Proposition 7.26 is effectively [33, Theorem 1.7] (replacing "crystalline" with "potentially crystalline" throughout). The nilpotent hypothesis was needed for Tannakian considerations, which in our case are obviated by Proposition 7.8.

Theorem 7.28 Given a Galois-equivariant quotient $R$ of ${ }^{\text {pcris }} \varpi_{f}^{\text {et }}\left(X_{\bar{K}}\right)$, the Galois action on $X_{\bar{K}}^{R \text {,ét }}$ is algebraic and potentially crystalline.

Proof In the notation of Section 5.3, we need to show that the map $\mathcal{G} \rightarrow \operatorname{Aut}^{h}\left(X_{\bar{K}}^{R}\right.$, ét $\left.{ }^{\text {,el }}\right)$ factors through $\mathcal{G}^{\text {pcris }}$. Apply Proposition 5.20 to Proposition 7.26, taking

$$
Y=\operatorname{Aut}^{h}\left(X \frac{R,{ }_{\bar{K}}, \mathrm{ét}}{}\right) \times_{\operatorname{Aut}(R)} \mathcal{G}^{\text {pcris, } 0}
$$

with the $\mathcal{G}^{0}$ action on $Y$ given by left multiplication.

Now, note that $D_{\text {pcris }}\left(\mathcal{G}^{\text {pcris, } 0} \times R\right)=D_{\text {pcris }}\left(\mathcal{G}^{\text {pcris, } 0}\right) \times R$, giving a $K_{0}^{\mathrm{nr}}$-linear map $f: D_{\text {pcris }}\left(\mathcal{G}^{\text {pcris, } 0}\right) \times R \rightarrow D_{\text {pcris }} R$. In fact,

$$
D_{\text {pcris }}\left(\mathcal{G}^{\text {pcris }, 0}\right)=\operatorname{Spec} B^{\operatorname{ker}\left(\mathcal{G}^{0} \rightarrow \mathcal{G}^{\text {pris }, 0}\right)},
$$

so this map just comes from the isomorphism $\left(D_{\text {pcris }} O(R)\right) \otimes_{K_{0}^{\mathrm{nr}}} B \cong O(R) \otimes_{\mathbb{Q}_{p}} B$. We now define $Z$ over $D_{\text {pcris }} \mathcal{G}^{\text {pcris, } 0}$ to be the affine scheme given by

$$
Z(A)=\operatorname{Iso}_{\mathrm{Ho}\left(d g \operatorname{Aff}_{A}(R)_{*}\right)}\left(X_{\bar{K}}^{R, \text { ét }}, \otimes_{\mathbb{Q}_{p}} A, f^{\sharp}\left(X_{\text {cris }}^{D_{\text {pris }} R, \text { Mal }} \otimes_{K_{0}^{\mathrm{nr}}} A\right)\right),
$$

for $D_{\text {pcris }} O\left(\mathcal{G}^{\text {pcris }, 0}\right)$-algebras $A$.

Since $\mathcal{G}^{\text {pcris, } 0}$ is potentially crystalline, we have an isomorphism $\alpha: \mathcal{G}^{\text {pcris, } 0} \times \operatorname{Spec} \widetilde{B} \rightarrow$ $\left(D_{\text {pcris }} \mathcal{G}^{\text {pcris, } 0}\right) \times_{\operatorname{Spec}} K_{0}^{\text {nr }} \operatorname{Spec} \widetilde{B}$, so the scheme $Z \times_{\operatorname{Spec}} K_{0}^{\text {nr }}$ Spec $\widetilde{B}$ can be regarded as a scheme over $\mathcal{G}^{\text {pcris, } 0} \times \operatorname{Spec} \widetilde{B}$

The $\mathcal{G}^{0}$-equivariant isomorphism of Proposition 7.26 then gives a $\mathcal{G}^{0}$-equivariant isomorphism

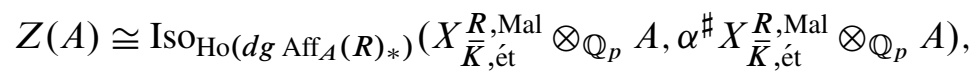

for any $D_{\text {pcris }} O\left(\mathcal{G}^{\text {pcris, } 0}\right) \otimes_{K_{0}^{\text {nr }}} \widetilde{B}$-algebra $A$, but the right-hand side is just $Y(A)$, giving a $\mathcal{G}^{0}$-equivariant isomorphism

$$
Z \times K_{0}^{\mathrm{nr}} \operatorname{Spec} \widetilde{B}_{\text {cris }} \cong Y \times \times_{\mathbb{Q}_{p}} \text { Spec } \widetilde{B}_{\text {cris }},
$$

as required. 
Corollary 7.29 For $x, y \in X(\bar{K})$, the $\mathcal{G}^{0}$-actions on

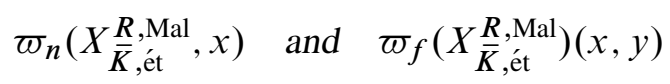

are potentially crystalline.

Proof This is just the observation that the map

$$
\operatorname{Aut}\left(X_{\bar{K}, \text { ét }}\right) \rightarrow \operatorname{Aut}\left(\varpi_{n}\left(X_{\bar{K}, \text {,ét }}^{R, \text { Mal }}, x\right)\right)\left(\mathbb{Q}_{p}\right)
$$

factors through $\operatorname{Aut}^{h}\left(X \frac{R, \text { Mal }}{\bar{K} \text {,ét }}\right)$.

Note that if we set $R=1$ and look at the fundamental group, this recovers the comparison theorem of $[48 ; 51]$ between pro-unipotent étale and crystalline fundamental groups.

In fact, we may extend Proposition 7.26 to a filtered version:

Proposition 7.30 For any Galois-equivariant quotient $R$ of ${ }^{\text {pcris }} \varpi_{f}^{\text {ét }}\left(X_{\bar{K}}\right)^{\text {red }}$ and for $j: X \rightarrow \bar{X}$, there is a chain of canonical $\left(\phi, \mathcal{G}^{0}\right)$-equivariant quasi-isomorphisms

$$
\left(X_{\bar{K} \text {,ét }}, j_{\bar{K} \text {,ét }}\right)^{R, \text { Mal }} \otimes_{\mathbb{Q}_{p}} \widetilde{B} \sim\left(X_{\bar{k} \text {,cris }}, j_{\bar{k}, \text { cris }}\right)^{D_{\text {pcris }}(R), \text { Mal }} \otimes_{K_{0}^{\text {nr }}} \widetilde{B}
$$

in $F s \operatorname{Aff}_{\widetilde{B}}(R)_{*}$.

Proof The proof of Proposition 7.26 adapts.

Lacking a suitable $p$-adic analogue of Lafforgue's Theorem (although [24, Theorem 6.3.4] might provide a viable replacement in some cases), we now impose a purity hypothesis.

Assumption 7.31 Assume that $D_{\text {pcris }}^{X} \mathbb{O}(R)$ is an ind-object in the category of $\iota$-pure overconvergent $F$-isocrystals. Like Definition 6.2, this is equivalent to saying that for every $R$-representation $V$, the corresponding sheaf $\mathbb{V}$ on $X_{\bar{K}}$ can be embedded in the pullback of a crystalline étale sheaf $\mathbb{U}$ on $X_{K^{\prime \prime}}$, associated to an $\iota$-pure overconvergent $F$-isocrystal on $\left(\bar{X}_{k^{\prime \prime}}, D_{k^{\prime \prime}}\right) / K^{\prime \prime}$, for some finite extension $K^{\prime} \subset K^{\prime \prime}$. Also note that this implies that the Frobenius action on $D_{\text {pcris }} O(R)$ is $\iota$-pure.

Example 7.32 To see how the hypotheses of Assumption 7.31 arise naturally, assume that $f: Y_{K} \rightarrow X_{K}$ is a geometric fibration (in the sense of [10, Definition 11.4], for instance any smooth proper morphism) with connected components, for $Y$ of potentially good reduction. Let $G(\bar{x}, \bar{z})$ be the Zariski closure of the map

$$
\pi_{f}^{\text {ét }}\left(X_{\bar{K}}\right)(\bar{x}, \bar{z}) \rightarrow \prod_{n} \operatorname{Iso}\left(\left(\mathbf{R}^{n} f_{\bar{K}, *}^{\text {ét }} \mathbb{Q}_{p}\right)_{\bar{x}},\left(\mathbf{R}^{n} f_{\bar{K}, *}^{\text {ét }} \mathbb{Q}_{p}\right)_{\bar{z}}\right)
$$


so $G$ is a pro-algebraic groupoid on objects $X(\bar{K})$, and then set $R=G^{\text {red }}$. By [8],

$$
\mathbf{R}^{n} f_{\bar{K}, *}^{\text {ét }} \mathbb{Q}_{p} \quad \text { is associated to } \quad \mathbf{R}^{n} f_{\bar{k}, *}^{\text {cris }} \mathrm{O}_{Y_{\bar{k}}} \text {,cris, }
$$

which by [24, Theorem 6.6.2] is $\iota$-pure (or if $f$ is not proper, globally $\iota$-mixed). Thus the semisimplifications of the $G$-representations

$$
\bar{x} \mapsto\left(\mathbf{R}^{n} f_{\bar{K}, *}^{\text {ét }} \mathbb{Q}_{p}\right)_{\bar{x}}
$$

are direct sums of $\iota$-pure representations. Since these generate the Tannakian category of $R$-representations, the hypotheses are satisfied.

For $\bar{x} \in X(\bar{K})$, we may write $F:=Y \times_{f, X, \bar{x}} \operatorname{Spec} \bar{K}$, and Theorem 3.32 then shows that the homotopy fibre of

$$
\left(Y_{\bar{K}}^{\text {ét }}\right)^{R, \text { Mal }} \rightarrow\left(X_{\bar{K}}^{\text {ét }}\right)^{R, \text { Mal }}
$$

over $\bar{x}$ is $\left(F_{\bar{K}}^{\text {ét }}\right)^{1, \mathrm{Mal}}$.

Example 7.33 A more comprehensive example would be to let $G(\bar{x}, \bar{z})$ be the Zariski closure of the map

$$
\pi_{f}^{\text {ét }}\left(X_{\bar{K}}\right)(\bar{x}, \bar{z}) \rightarrow \prod_{n, f} \operatorname{Iso}\left(\left(\mathbf{R}^{n} f_{\bar{K}, *}^{\text {ét }} \mathbb{Q}_{p}\right)_{\bar{x}},\left(\mathbf{R}^{n} f_{\bar{K}, *}^{\text {ét }} \mathbb{Q}_{p}\right)_{\bar{z}}\right)
$$

where $f$ ranges over all geometric fibrations of potentially good reduction with connected components, and then to set $R:=G^{\text {red }}$. The resulting homotopy type $\left(X_{\bar{K}}^{\text {ét }}\right)^{R, \text { Mal }}$ would be very close to possible conceptions of a pro-algebraic motivic homotopy type.

Theorem 7.34 Given a Galois-equivariant quotient $R$ of ${ }^{\text {pcris }} \varpi_{f}^{\text {ét }}\left(X_{\bar{K}}\right)$ satisfying Assumption 7.31, the Galois action on $X_{\bar{K}}^{R}$, Mal is $\iota$-mixed in the sense of Definition 5.22, giving a canonical weight decomposition on $X_{\bar{K}}^{R}$, ,ét $B^{\sigma}$.

Proof This is essentially the same as Proposition 6.6. Frobenius gives a canonical element of $\operatorname{Aut}^{h}\left(X_{\text {cris }}^{D_{\text {pcris }} R, \mathrm{Mal}}\right)$. We first show that this is $\iota$-mixed of integral weights. By Lemma 4.9, we need only consider the Frobenius action on cohomology

$$
\mathrm{H}_{\text {cris }}^{*}\left(\left(\bar{X}_{\bar{k}}, D_{\bar{k}}\right), D_{\text {pcris }}^{X} \mathbb{O}(R)\right) \text {. }
$$

The Leray spectral sequence gives

$$
\mathrm{H}_{\text {cris }}^{2 a+b}\left(\bar{X}_{\bar{k}}, \mathbf{R}_{\text {cris }}^{-a} j_{*} D_{\text {pcris }}^{X} \mathbb{O}(R)\right) \Longrightarrow \mathrm{H}_{\text {cris }}^{a+b}\left(\left(\bar{X}_{\bar{k}}, D_{\bar{k}}\right), D_{\text {pcris }}^{X} \mathbb{O}(R)\right) \text {. }
$$


If we write $D^{(n)}$ for the normalisation of the $n$-fold intersection of the local components of $D$, and $i_{n}: D^{(n)} \rightarrow \bar{X}$ for the embedding, then as in [4, 3.2.4.1], there is an isomorphism

$$
\mathrm{H}_{\text {cris }}^{2 a+b}\left(\bar{X}_{\bar{k}}, \mathbf{R}_{\text {cris }}^{-a} j_{*} D_{\text {pcris }}^{X} \mathbb{O}(R)\right) \cong \mathrm{H}_{\text {cris }}^{2 a+b}\left(D_{\bar{k}}^{(-a)}, i_{n}^{*} j_{*} D_{\text {pcris }}^{X} \mathbb{O}(R)(a)\right),
$$

since $j_{*} D_{\text {pcris }}^{X} \mathbb{O}(R)$ is associated to a locally constant sheaf on $X$.

Now, [24, Theorem 6.6.2] combined with Poincaré duality proves that

$$
\mathrm{H}_{\text {cris }}^{2 a+b}\left(D_{\bar{k}}^{(-a)}, i_{n}^{*} j_{*} D_{\text {pcris }}^{X} \mathbb{O}(R)(a)\right)
$$

is $\iota$-pure of weight $b$. Thus Lemma 4.9 implies that the Frobenius element of $\operatorname{Aut}^{h}\left(X_{\text {cris }}^{D_{\text {pris }} R, \mathrm{Mal}}\right)$ is $\iota$-mixed of integral weights.

We need to show that the composite morphism

$$
\mathbb{Z}^{\mathrm{alg}, 0} \rightarrow \mathcal{G}^{\text {pcris }} \otimes \mathbb{Q}_{p} B^{\sigma} \rightarrow \operatorname{Aut}^{h}\left(X_{\bar{K}, \text {,ét }}^{R \text { al }}\right) \otimes \mathbb{Q}_{p} B^{\sigma}
$$

factors through $M_{\iota}^{0}$. By Proposition 7.26,

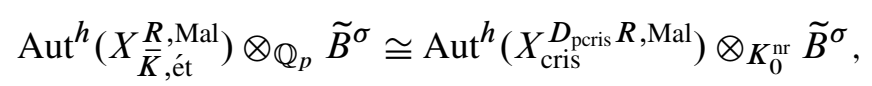

so the map

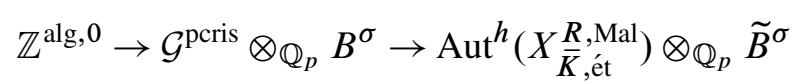

factors through $M_{\iota}^{0}$. Since $B^{\sigma} \subset \widetilde{B}^{\sigma}$, this completes the proof.

Theorem 7.35 For $R$ as in Theorem 7.34, the filtered homotopy type

$$
\left(X_{\bar{K} \text {,ét }}, j_{\bar{K} \text {,ét }}\right)^{R, \text { Mal }} \otimes B^{\sigma}
$$

is quasiformal, corresponding to the $E_{2}$-term

$$
{ }_{J} \mathrm{E}_{1}^{a, b}\left(X \bar{K}_{\bar{K}, \mathrm{ét}}^{R, \mathrm{Mal}^{\prime}}\right) \otimes B^{\sigma}=\bigoplus_{a, b} \mathrm{H}^{2 a+b}\left(\bar{X}_{\bar{K}}, \mathbf{R}^{-b} j_{*} \mathbb{O}(R)\right) \otimes B^{\sigma} \in F D G \operatorname{Alg}_{B^{\sigma}}(R),
$$

of the Leray spectral sequence for the immersion $j: X \rightarrow \bar{X}$, and the formality isomorphism is equivariant with respect to the Galois action.

The filtered homotopy type $\left(X_{\bar{K}} \text {,ét }, j_{\bar{K}} \text {,ét }\right)^{R, \text { Mal }}$ is also quasiformal, but the formality isomorphism is not in general Galois-equivariant or canonical.

Proof Since the Galois action is $\iota$-mixed in the sense of Definition 5.22, there is a Galois-equivariant weight decomposition $\mathbb{G}_{m} \rightarrow \operatorname{RAut}_{J}\left(X_{\bar{K}}^{R}\right.$, Mal $\left.{ }_{\text {, }} \otimes B^{\sigma}\right)$, using Lemma 5.21 and the observation after Definition 5.3. The argument of Corollary 6.15 
now adapts to show that $X_{\bar{K}}^{R}$, ét $\otimes B^{\sigma}$ is quasiformal, with the formality quasiisomorphism equivariant under the Galois action, proving the first part.

In particular this implies that

$$
\operatorname{RAut}_{J}\left(X_{\bar{K}, \text { ét }}^{R, \mathrm{Mal}}\right)\left(B^{\sigma}\right) \rightarrow \operatorname{Aut}\left(J \mathrm{E}_{1}^{*, *}\left(X_{\bar{K}}^{R, \mathrm{Mal}^{\prime}}\right)\right)\left(B^{\sigma}\right)
$$

is a pro-unipotent extension. Thus the corresponding morphism of pro-algebraic groups is surjective, which allows to lift the weight decomposition on $\mathrm{E}_{1}^{*, *}\left(X_{\bar{K}}^{R}, \mathrm{Mal}\right)$ noncanonically to $X_{\bar{K}}$,ét . This decomposition is not necessarily compatible with the canonical decomposition on $X_{\bar{K}}^{R}$, ét $\otimes B^{\sigma}$. The argument of Corollary 6.15 adapted to this decomposition now shows that $X \underset{\bar{K}}{R \text {,ét }}$, is quasiformal.

Corollary 7.36 For $X$ and $R$ as above, we can describe the homotopy groups $\varpi_{n}^{\text {ét }}\left(X_{\bar{K}}^{R}\right.$, Mal,$\left.x\right) \times_{\text {Spec } \mathbb{Q}_{p}}$ Spec $B^{\sigma}$ explicitly in terms of the Leray spectral sequence as

$$
\varpi_{n}^{\text {ét }}\left(X_{\bar{K}}^{R, \text { Mal }}, x\right)^{\vee} \otimes_{\mathbb{Q}_{p}} B^{\sigma}=\mathrm{H}^{n-1}\left(G\left(J \mathrm{E}_{1}^{*, *}\left(X_{\bar{K} \text {,ét }}^{R, \mathrm{Mal}}\right)\right)^{\vee}\right) \otimes_{\mathbb{Q}_{p}} B^{\sigma},
$$

for $G$ as in Definition 4.20. Of course, if the conditions of Theorem 3.40 hold (including goodness), then this also calculates $\pi_{n}^{\text {ét }}\left(X_{\bar{K}}, x\right) \otimes_{\widehat{\mathbb{Z}}} B^{\sigma}$ as a Galois representation.

Remarks 7.37 (1) In the case when $X$ is projective and $R$ is a quotient of ${ }^{\mathrm{Gal}} \varpi_{f}\left(X_{\bar{k}}\right)$, this is essentially the main formality result of [32, Section 4], which has since been extended to the general projective case in [33, Theorem 7.22], although Frobenius-equivariance is not made explicit there. The proofs also differ in that they work with minimal algebras, rather than minimal Lie algebras.

(2) Although at first sight Theorem 7.35 is weaker than Theorem 7.3, it is more satisfactory in one important respect. Theorem 7.3 effectively shows that relative Malcev $\ell$-adic homotopy types carry no more information than cohomology, whereas to recover a relative Malcev $p$-adic homotopy type from Theorem 7.35, we still need to identify

$$
\left(X_{\bar{K} \text {,ét }}, j_{\bar{K} \text {,ét }}\right)^{R, \text { Mal }} \subset\left(X_{\bar{K} \text {,ét }} j_{\bar{K} \text {,ét }}\right)^{R, \text { Mal }} \otimes B^{\sigma} .
$$

This must be done by describing the Hodge filtration on $\left(X_{\text {cris }}^{D_{\text {pris }} R, \text { Mal }}, j_{\bar{k}}\right.$,cris $)$, which is not determined by cohomology (since it is not Frobenius-equivariant). Thus the Hodge filtration is the only really new structure on the relative Malcev homotopy type. This phenomenon is similar to the formality results for mixed Hodge structures in [34, Section 2]. 


\section{References}

[1] F Andreatta, A Iovita, Comparison isomorphisms for smooth formal schemes, Preprint (2009) Available at http://www.mathstat.concordia.ca/faculty/iovita/ research.html

[2] M Artin, B Mazur, Etale homotopy, Lecture Notes in Math. 100, Springer, Berlin (1969) MR0245577

[3] A K Bousfield, D M Kan, Homotopy limits, completions and localizations, Lecture Notes in Math. 304, Springer, Berlin (1972) MR0365573

[4] P Deligne, Théorie de Hodge. II, Inst. Hautes Études Sci. Publ. Math. (1971) 5-57 MR0498551

[5] P Deligne, La conjecture de Weil. II, Inst. Hautes Études Sci. Publ. Math. (1980) 137-252 MR601520

[6] P Deligne, J S Milne, A Ogus, K-y Shih, Hodge cycles, motives, and Shimura varieties, Lecture Notes in Math. 900, Springer, Berlin (1982) MR654325

[7] W G Dwyer, D M Kan, Homotopy theory and simplicial groupoids, Nederl. Akad. Wetensch. Indag. Math. 46 (1984) 379-385 MR770723

[8] G Faltings, Crystalline cohomology and p-adic Galois representations, from: "Algebraic analysis, geometry, and number theory (Baltimore, MD, 1988)", (J-I Igusa, editor), Johns Hopkins Univ. Press, Baltimore, MD (1989) 25-80 MR1463696

[9] J-M Fontaine, Sur certains types de représentations p-adiques du groupe de Galois d'un corps local; construction d'un anneau de Barsotti-Tate, Ann. of Math. (2) 115 (1982) 529-577 MR657238

[10] E M Friedlander, Étale homotopy of simplicial schemes, Annals of Math. Studies 104, Princeton Univ. Press (1982) MR676809

[11] PG Goerss, JF Jardine, Simplicial homotopy theory, Progress in Math. 174, Birkhäuser Verlag, Basel (1999) MR1711612

[12] A Grothendieck, Revêtements étales et groupe fondamental (SGA 1), Documents Math. (Paris) 3, Soc. Math. France (2003) MR2017446 Séminaire de géométrie algébrique du Bois Marie 1960-61, With two papers by M Raynaud, Updated and annotated reprint of the 1971 original [Lecture Notes in Math. 224, Springer, Berlin; MR0354651]

[13] R M Hain, The Hodge de Rham theory of relative Malcev completion, Ann. Sci. École Norm. Sup. (4) 31 (1998) 47-92 MR1604294

[14] R M Hain, M Matsumoto, Relative pro-l completions of mapping class groups, J. Algebra 321 (2009) 3335-3374 MR2510052

[15] V A Hinich, V V Schechtman, On homotopy limit of homotopy algebras, from: “ $K-$ theory, arithmetic and geometry (Moscow, 1984-1986)", (Y I Manin, editor), Lecture Notes in Math. 1289, Springer, Berlin (1987) 240-264 MR923138 
[16] PS Hirschhorn, Model categories and their localizations, Math. Surveys and Monogr. 99, Amer. Math. Soc. (2003) MR1944041

[17] G Hochschild, G D Mostow, Pro-affine algebraic groups, Amer. J. Math. 91 (1969) 1127-1140 MR0255690

[18] M Hovey, Model categories, Math. Surveys and Monogr. 63, Amer. Math. Soc. (1999) MR1650134

[19] D C Isaksen, A model structure on the category of pro-simplicial sets, Trans. Amer. Math. Soc. 353 (2001) 2805-2841 MR1828474

[20] U Jannsen, Continuous étale cohomology, Math. Ann. 280 (1988) 207-245 MR929536

[21] N M Katz, p-adic properties of modular schemes and modular forms, from: "Modular functions of one variable, III (Proc. Internat. Summer School, Univ. Antwerp, Antwerp, 1972)", (W Kuyk, J-P Serre, editors), Lecture Notes in Math. 350, Springer, Berlin (1973) 69-190 MR0447119

[22] L Katzarkov, T Pantev, B Toën, Schematic homotopy types and non-abelian Hodge theory, Compos. Math. 144 (2008) 582-632 MR2422341

[23] L Katzarkov, T Pantev, B Toën, Algebraic and topological aspects of the schematization functor, Compos. Math. 145 (2009) 633-686 MR2507744

[24] KS Kedlaya, Fourier transforms and p-adic 'Weil II', Compos. Math. 142 (2006) 1426-1450 MR2278753

[25] R Kiehl, R Weissauer, Weil conjectures, perverse sheaves and l'adic Fourier transform, Ergebnisse der Math. und ihrer Grenzgebiete. 3. Folge. 42, Springer, Berlin (2001) MR1855066

[26] M Kontsevich, Topics in algebra - deformation theory, Lecture notes (1994) Available at http://www.math.brown.edu/ abrmovic/kontsdef.ps

[27] L Lafforgue, Chtoucas de Drinfeld et correspondance de Langlands, Invent. Math. 147 (2002) 1-241 MR1875184

[28] S Mac Lane, Categories for the working mathematician, Graduate Texts in Math. 5, Springer, New York (1971) MR0354798

[29] A R Magid, On the proalgebraic completion of a finitely generated group, from: "Combinatorial and geometric group theory (New York, 2000/Hoboken, NJ, 2001)", (S Cleary, R Gilman, A G Myasnikov, V Shpilrain, editors), Contemp. Math. 296, Amer. Math. Soc. (2002) 171-181 MR1921711

[30] J S Milne, Étale cohomology, Princeton Math. Series 33, Princeton Univ. Press (1980) MR559531

[31] J W Morgan, The algebraic topology of smooth algebraic varieties, Inst. Hautes Études Sci. Publ. Math. (1978) 137-204 MR516917 
[32] M C Olsson, F-isocrystals and homotopy types, J. Pure Appl. Algebra 210 (2007) 591-638 MR2324594

[33] M C Olsson, Towards non-abelian p-adic Hodge theory in the good reduction case, Mem. Amer. Math. Soc. 210 no. 990, Amer. Math. Soc. (2011)

[34] J P Pridham, Formality and splitting of real non-abelian mixed Hodge structures arXiv:0902 .0770v2

[35] J P Pridham, Galois actions on the pro-l-unipotent fundamental group arXiv: math.AG/0404314

[36] J P Pridham, Deforming l-adic representations of the fundamental group of a smooth variety, J. Algebraic Geom. 15 (2006) 415-442 MR2219844

[37] J P Pridham, Pro-algebraic homotopy types, Proc. Lond. Math. Soc. (3) 97 (2008) 273-338 MR2439664

[38] J P Pridham, Weight decompositions on étale fundamental groups, Amer. J. Math. 131 (2009) 869-891 MR2530856

[39] J P Pridham, The homotopy theory of strong homotopy algebras and bialgebras, Homology, Homotopy Appl. 12 (2010) 39-108 MR2721031

[40] J P Pridham, Unifying derived deformation theories, Adv. Math. 224 (2010) 772-826 MR2628795

[41] J P Pridham, $\ell$-adic pro-algebraic and relative pro- $\ell$ fundamental groups, to appear in "The arithmetic of fundamental groups (PIA 2010)", (J Stix, editor), Springer, Berlin (2011) Available at http://www.dpmms.cam.ac.uk/ jpp24/heid.pdf

[42] G Quick, Profinite homotopy theory, Doc. Math. 13 (2008) 585-612 MR2466189

[43] D Quillen, An application of simplicial profinite groups, Comment. Math. Helv. 44 (1969) 45-60 MR0242156

[44] D Quillen, Rational homotopy theory, Ann. of Math. (2) 90 (1969) 205-295 MR0258031

[45] A Schmidt, Extensions with restricted ramification and duality for arithmetic schemes, Compositio Math. 100 (1996) 233-245 MR1383466

[46] J-P Serre, Lie algebras and Lie groups, second edition, Lecture Notes in Math. 1500, Springer, Berlin (1992) MR1176100 1964 lectures given at Harvard University

[47] J-P Serre, Cohomologie galoisienne, fifth edition, Lecture Notes in Math. 5, Springer, Berlin (1994) MR1324577

[48] A Shiho, Crystalline fundamental groups and p-adic Hodge theory, from: "The arithmetic and geometry of algebraic cycles (Banff, AB, 1998)", (B B Gordon, J D Lewis, S Müller-Stach, S Saito, N Yui, editors), CRM Proc. Lecture Notes 24, Amer. Math. Soc. (2000) 381-398 MR1738868

[49] B Toën, Champs affines, Selecta Math. (N.S.) 12 (2006) 39-135 MR2244263 
[50] T Tsuji, Crystalline sheaves, syntomic cohomology and p-adic polylogarithms, Caltech seminar notes (2001) Available at http://modular.math.washington.edu/ swc/notes/files/DLSTsuji2.pdf

[51] V Vologodsky, Hodge structure on the fundamental group and its application to $p$-adic integration, Mosc. Math. J. 3 (2003) 205-247, 260 MR1996809

[52] C A Weibel, An introduction to homological algebra, Cambridge Studies in Advanced Math. 38, Cambridge Univ. Press (1994) MR1269324

Department of Pure Mathematics and Mathematical Statistics, University of Cambridge Wilberforce Road, Cambridge, CB3 OWB, UK

J.P.Pridham@dpmms . cam.ac.uk

http://www.dpmms.cam.ac.uk/ jpp24/

Proposed: Haynes Miller

Received: 10 December 2009

Seconded: Bill Dwyer, Paul Goerss

Revised: 27 January 2011 